January 2004 - NREL/TP-500-34593

\title{
Lamar Low-Level Jet Project Interim Report
}

N. Kelley, M. Shirazi, D. Jager, S. Wilde, J. Adams, and M. Buhl National Renewable Energy Laboratory

P. Sullivan and E. Patton

National Center for Atmospheric Research

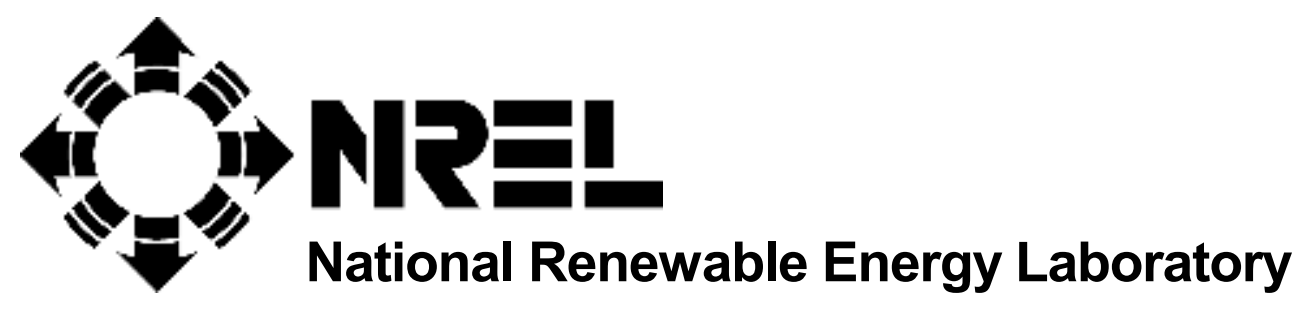

1617 Cole Boulevard Golden, Colorado 80401-3393

NREL is a U.S. Department of Energy Laboratory Operated by Midwest Research Institute • Battelle Contract No. DE-AC36-99-G010337 


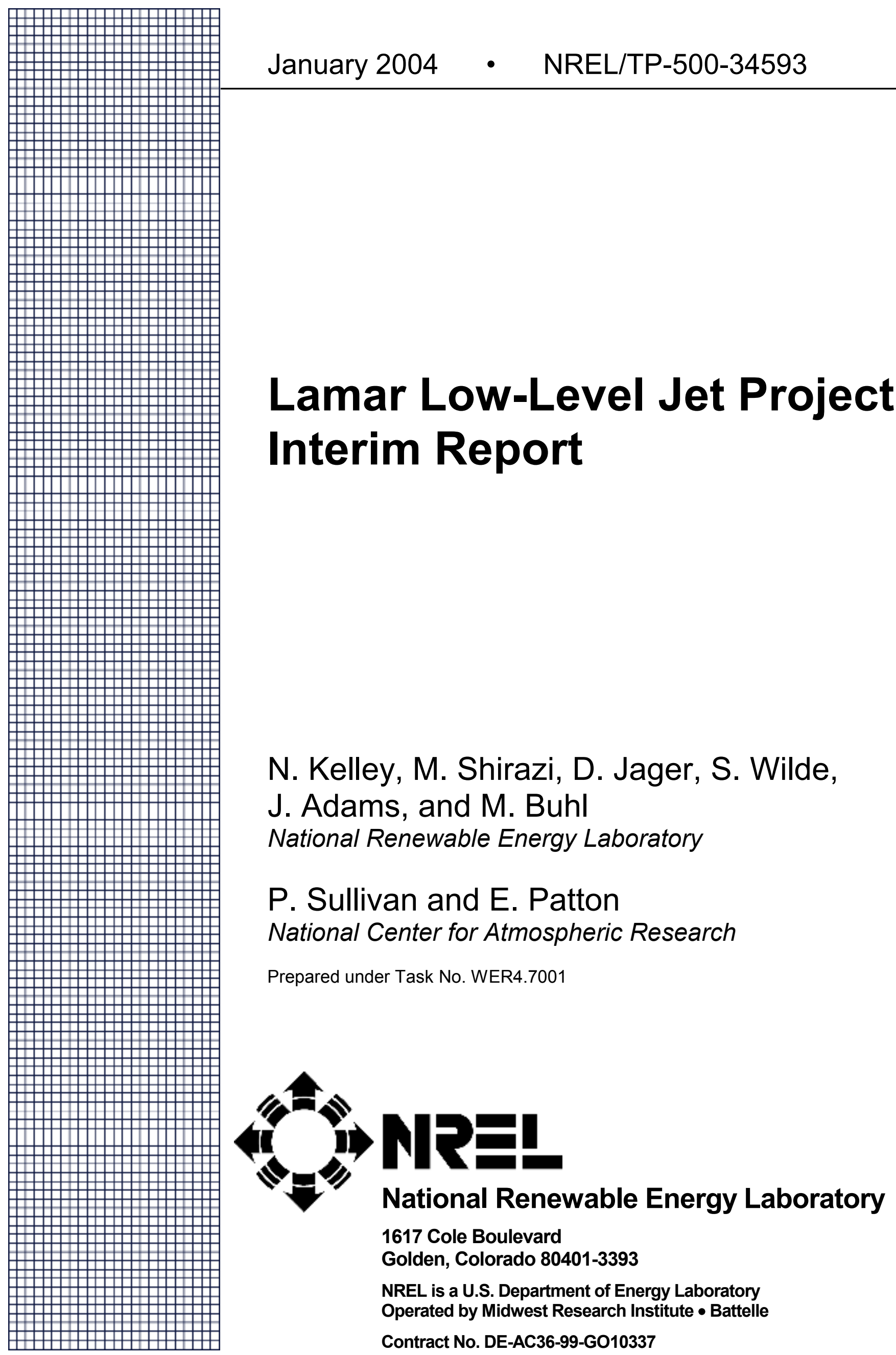




\section{NOTICE}

This report was prepared as an account of work sponsored by an agency of the United States government. Neither the United States government nor any agency thereof, nor any of their employees, makes any warranty, express or implied, or assumes any legal liability or responsibility for the accuracy, completeness, or usefulness of any information, apparatus, product, or process disclosed, or represents that its use would not infringe privately owned rights. Reference herein to any specific commercial product, process, or service by trade name, trademark, manufacturer, or otherwise does not necessarily constitute or imply its endorsement, recommendation, or favoring by the United States government or any agency thereof. The views and opinions of authors expressed herein do not necessarily state or reflect those of the United States government or any agency thereof.

Available electronically at http://www.osti.gov/bridge

Available for a processing fee to U.S. Department of Energy and its contractors, in paper, from:

U.S. Department of Energy

Office of Scientific and Technical Information

P.O. Box 62

Oak Ridge, TN 37831-0062

phone: 865.576 .8401

fax: 865.576.5728

email: reports@adonis.osti.gov

Available for sale to the public, in paper, from:

U.S. Department of Commerce

National Technical Information Service

5285 Port Royal Road

Springfield, VA 22161

phone: 800.553.6847

fax: 703.605.6900

email: orders@ntis.fedworld.gov

online ordering: http://www.ntis.gov/ordering.htm 


\section{Acknowledgments}

This work is supported by the U.S. Department of Energy under contract no. DE-AC3683CH10093. The authors wish to thank the Office of Wind and Hydropower Technologies for the support of this program.

The authors also wish to thank GE Wind Energy for their support of this partnership by providing the meteorological tower and site support for this effort. We particularly salute the efforts of Craig Christenson and Emil Moroz for their vision in developing the objectives of this program and their continued support.

The authors want to specifically acknowledge the outstanding contribution to this project by our recently retired team member and colleague Ed McKenna. The success of the field operations is a testament to Ed's outstanding planning, technical, and supervisory skills, which our team was able to build upon.

The authors particularly want to acknowledge the wonderful support and cooperation afforded us the Emick Family. Without the assistance of Bob and Helen, Kenny and Kelly, and Greg and Valerie Emick we would not have been as successful as we were. The Emicks have been willing to assist us numerous times on short notice and have adjusted their ranch operations on occasion to support this important effort.

The authors wish to thank Professor Robert E. Wilson of the Oregon State University and Dr. Alfred J. Bedard, Jr., of the National Oceanic and Atmospheric Administration for their discussions and very valuable comments. 


\section{Table of Contents}

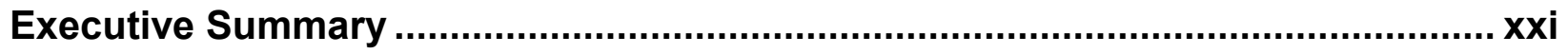

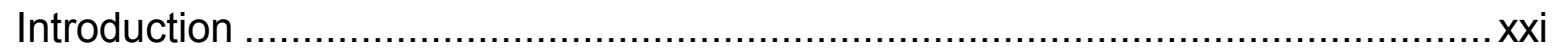

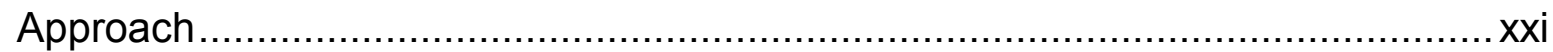

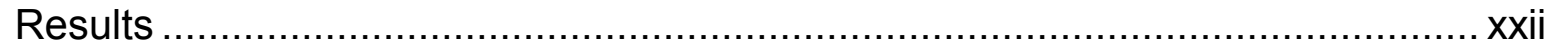

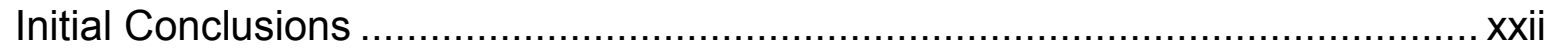

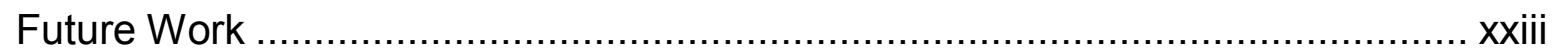

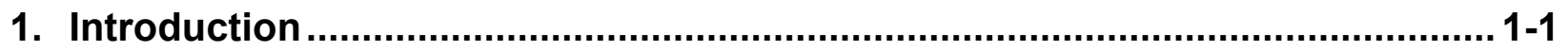

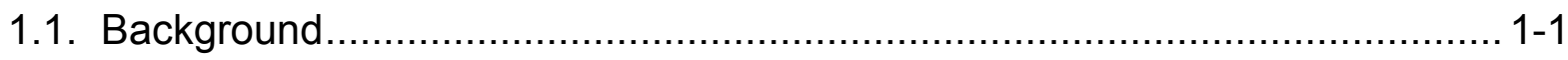

1.2. Turbulence-induced Turbine Structure Response - Previous Studies............ 1-1

1.2.1. San Gorgonio Micon 65/13 ….................................................. 1-2

1.2.2. NWTC CWE300 Wind Eagle .................................................... 1-4

1.2.3. NWTC/LIST Program................................................................ 1-4

1.2.4. Role of Coherent Inflow Turbulence in Inducing Turbine

Aeroelastic and Structural Response ............................................... 1-5

1.2.5. Atmospheric Sources of Coherent Structures Affecting Wind Turbines .. 1-6

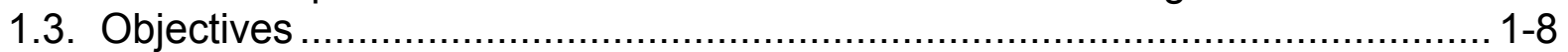

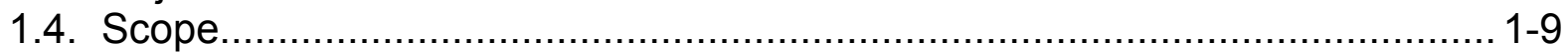

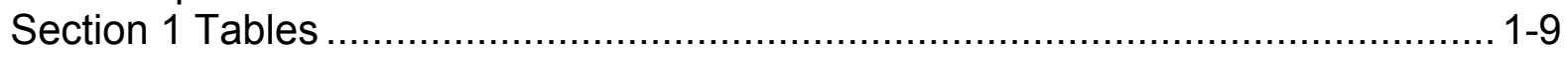

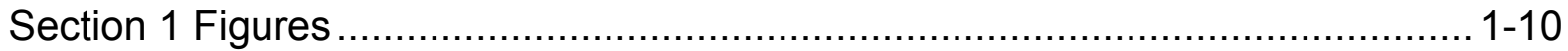

2. An Overview of the Atmospheric Boundary Layer ....................................... 2-1

2.1. Daytime or Convective Boundary Layer................................................ 2-2

2.2. Neutral Boundary Layer ................................................................... 2-2

2.3. Nocturnal or Stable Boundary Layer .................................................. 2-2

2.4. Nocturnal Low-Level Jet Streams .................................................... 2-2

2.4.1. Low-Level Jet Climatology ……................................................... 2-3

2.4.2. Theory of Low-Level Jet Formation ................................................ 2-3

2.5. Atmospheric Wave Motions ...................................................................... 2-4

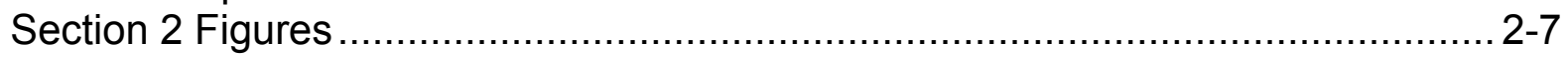

3. Analysis of NWTC/LIST Program Results ..................................................... 3-1

3.1. A Simple Linear-Regression Model of ART Turbulence-Induced Response... 3-1

3.1.1. Identification of Inflow (Excitation) and Response Variable ................... 3-1

3.1.2. The Stepwise Regression Results ..................................................... 3-1

3.2. Interpretation of NWTC/LIST Results.................................................. 3-3

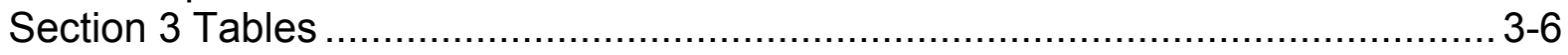

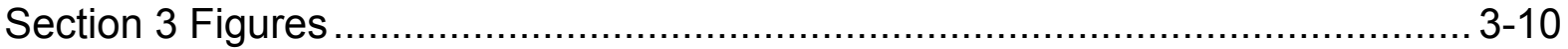


4. The Lamar Low-Level Jet Program ............................................................ 4-1

4.1. Instrumentation and Data Collection .................................................... 4-1

4.1.1. Tower-based Instrumentation .................................................. 4-2

4.1.2. Acoustic Wind Profiler (SODAR) ................................................ 4-2

4.1.3. Data Acquisition ................................................................... 4-3

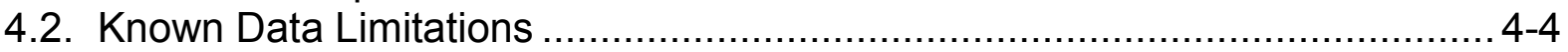

4.2.1. Tower-based Measurement Limitations ........................................... 4-4

Temporary tower data losses.................................................... 4-4

Systemic tower measurement issues............................................... 4-6

4.2.2. SODAR Measurement Limitations ................................................ 4-7

4.3. Future Measurements ….................................................................. 4

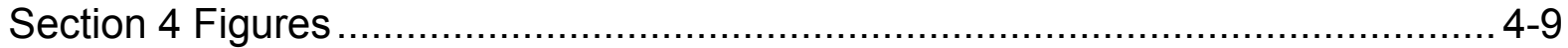

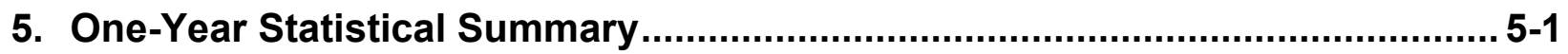

5.1. Basic Meteorological Background Parameters ........................................ 5-1

5.1.1. Atmospheric Thermodynamics .................................................... 5-1

5.1.2. Wind Energy, Shear, and Turbulence ........................................... 5-1

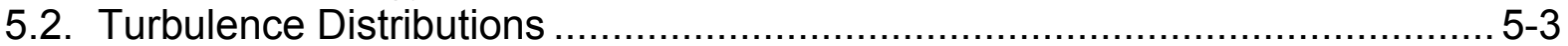

5.2.1. Turbulent Intensity Distributions .................................................. 5-3

5.2.2. Turbulence Level (Wind Speed Standard Deviation) Distributions ......... 5-3

5.2.3. Gust Distributions ....................................................................... 5-4

5.3. Vertical Shear Distributions.............................................................. 5-4

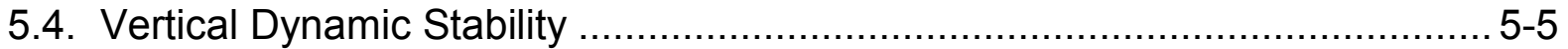

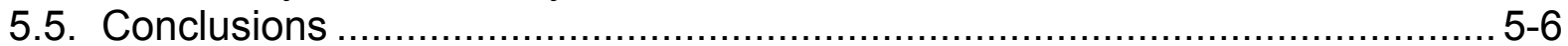

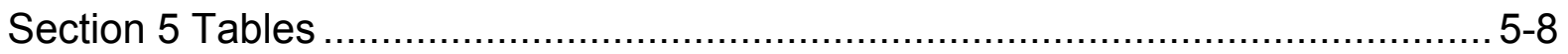

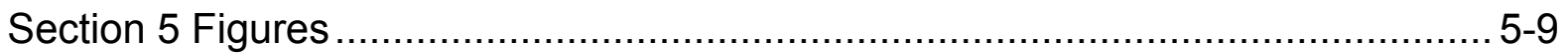

6. Three Representative Case Studies …............................................................. 6-1

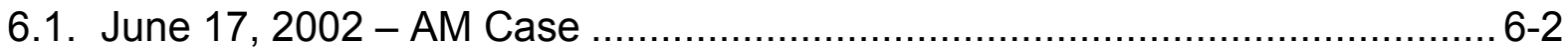

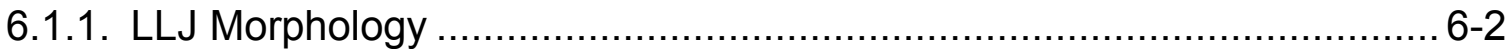

6.1.2. Vertical Shear Characteristics................................................... 6-2

6.1.3. Turbulence Environment and Potential Turbine Impacts ..................... 6-3

6.2. June 17, 2002 - PM Case ............................................................. 6-5

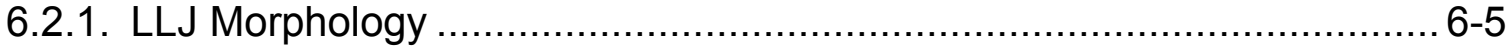

6.2.2. Vertical Shear Characteristics................................................... 6-5

6.2.3. Turbulence Environment........................................................ 6-6

6.2.4. Potential Turbine Impacts ........................................................ 6-10

6.3. June 23, 2002 - AM Case ............................................................... 6-10

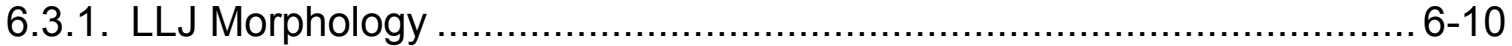

6.3.2. Vertical Shear Characteristics.................................................... 6-11

6.3.3. Turbulence and Potential Turbine Impacts ......................................6-11

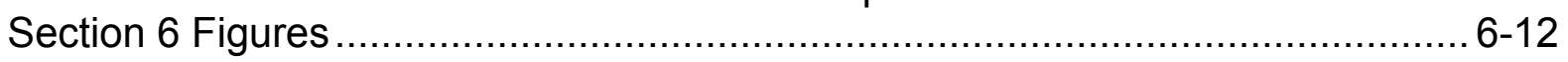


7. Using NWTC/LIST Results to Estimate Turbine Response .............................. 7-1

7.1. Summary of Relationships Between Turbulent Structural

Parameters and Turbine Response for LLLJPP Case Studies........................ 7-1

7.2. Estimating Turbine Response at LLLJP Site............................................. 7-2

7.2.1. NWTC/LIST and LLLJP Case Studies Predictor Variable Distributions .. 7-2

7.2.2. Comparison of Predicted ART Response at LLLJP Site with NWTC ......7-3

Section 7 Figures ............................................................................ $7-5$

8. Interaction of WINDPACT Turbine with a KH Billow ........................................ 8-1

8.1. The WindPACT 1.5-MW Baseline Turbine Test Bed ................................... 8-1

8.2. Interfacing the NCAR KH Billow Simulation to the AeroDyn Code................. 8-1

8.3. Example of Using NCAR KH Billow as Input to Turbine Dynamic Simulation . 8-2

8.3.1. Simulated Inflow Description......................................................... 8-3

8.3.2. Response of Turbine to KH Billow.......................................................... 8-4

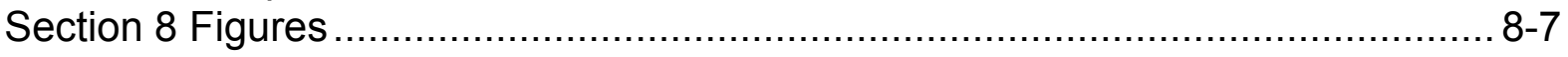

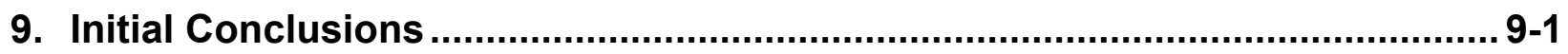

9.1. NWTC/LIST Program........................................................................ 9-1

9.2. One-Year LLLJP Statistical Data Base Analysis ...................................... 9-1

9.3. Case Study Results ...................................................................... 9-2

9.4. Interaction of a Turbine Rotor Blade with a KH Billow................................. 9-3

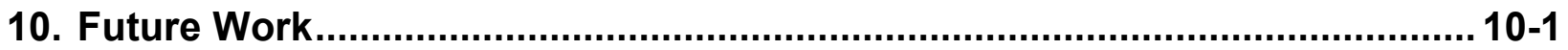

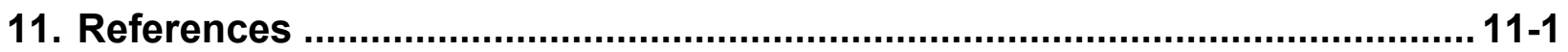




\section{List of Figures}

\section{Chapter 1}

Figure 1-1. Variation of hub-height local shear stress $\left(u_{*}\right)$ and root flapwise high-loading tail shape parameter for Micon 65/13 turbines in San Gorgonio Pass wind farm

Figure 1-2. Variation of hub-height local shear stress $\left(u_{*}\right)$ and root edgewise high-loading tail shape parameter for Micon 65/13 turbines in San Gorgonio Pass wind farm

Figure 1-3. Time and wavelet analysis of CWE300 turbine turbulence response

Figure 1-4. Continuous wavelet transform analysis of turbulence excitation for CWE300 turbine

Figure 1-5. Time and wavelet analysis of simulated CWE300 turbine turbulence/rotor interaction

Figure 1-6. Continuous wavelet transform analysis of simulated CWE300 turbine turbulence/rotor interaction.

Figure 1-7. Diurnal variation of three-blade average flapwise root equivalent loads $L_{e q}$ for Micon 65/13 turbines in San Gorgonio Pass wind farm

Figure 1-8. Variation of hub-height friction velocity $u_{*}$ and three-blade average flapwise equivalent loads $L_{e q}$ versus turbine layer gradient Richardson number (Ri) for Micon 65/13 turbine in San Gorgonio Pass wind farm

Figure 1-9. Time distribution of Micon 65/13 turbines peak loads by indicated groups

Figure 1-10. Diurnal variation of inflow Richardson number $(R i)$ and hub-height friction velocity $u_{*}$ for Micon 65/13 turbines in San Gorgonio Pass wind farm

Figure 1-11. Diurnal fault and shear patterns at Big Spring, Texas (Source: K. Smith et al. [11])

\section{Chapter 2}

Figure 2-1. Example of low-level jet evolution from SODAR-derived 10-minute mean wind speed and direction profiles for LLLJP Site on 16-17 June 2002

Figure 2-2. Overlay of biannual low-level jet frequency and wind resource map for eastern 2/3 of the U.S. (Low-level jet data source: Bonner [14])

Figure 2-3. Non-dimensional temperature field of $\mathrm{KH}$ billow life cycle 2-9 


\section{Chapter 3}

Figure 3-1. Variation of observed inflow parameters during NWTC/LIST experiment October 28, 2000, to May 17, 2001

Figure 3-2. Variation of observed flapwise $L_{e q}$ versus inflow parameters during NWTC/LIST experiment

Figure 3-3. Variation of ART turbine root flapwise equivalent loads $L_{e q}$ and hub-height vertical wind speed standard deviation $\sigma_{w}$ and peak coherent turbulent kinetic energy $E_{c o h}$ as a function of the turbine-layer gradient Richardson number Ri for NWTC/LIST experiment.

Figure 3-4. Variation of turbine-layer buoyancy length scale $I_{b}$ normalized by the ART turbine rotor diameter $D$ or $I_{b} / D$ for NWTC/LIST experiment.

Figure 3-5. Time history example of a coherent turbulent structure in a octurnal stably stratified flow and the corresponding response of the ART turbine

Figure 3-6. Example of variation of the downward flux of turbulent kinetic energy $E$ recorded by the NWTC/LIST upwind array of sonic anemometers

\section{Chapter 4}

Figure 4-1. GE Wind 120-m met tower - southeast Colorado (LLLJP Site)........ 4-9

Figure 4-2. Close-up of NREL turbulence measuring instrumentation................ 4-9

Figure 4-3. Scintec Model MFAS SODAR antenna .................................... 4-10

Figure 4-4. Installation and plan view of Scintec SODAR near GE Wind 120-m tower

Figure 4-5. ATI sonic block-averaging filter frequency transfer function (200:20 frequency reduction).

Figure 4-6. Aeolian vortex shedding frequencies as a function of wind speed from structural elements of GE Wind 120-m tower

Figure 4-7. Example of vertical wind structures as resolved by NOAA HighResolution Doppler LIDAR (HRDL)

(Source: R. Banta, NOAA/ETL)

\section{Chapter 5}

Figure 5-1. Annual variation of monthly mean temperatures, layer mean temperatures, and mean relative humidity from GE Wind 120-m tower for period October 2002 through October 2003

Figure 5-2. Annual variation of monthly mean air densities, layer mean air densities, and 3-m barometric pressure from GE Wind 120-m tower for period October 2001 through October 2002 
Figure 5-3. Annual variation of monthly normalized energy density, height energy density ratio, and turbulence intensity from GE Wind 120-m tower for period October 2001 through October 2002

Figure 5-4. Annual variation of monthly mean wind directions, normalized layer mean energy densities, and layer mean shear exponents from GE Wind 120-m tower for period October 2001 through October 2002

Figure 5-5. Annual joint probability distributions for the mean wind speed at the 52- to 113-m layer and the 52- to 113-m layer Ri

Figure 5-6. Annual joint probability distributions of turbulence intensities and mean wind speed

Figure 5-7. Annual joint probability distributions of turbulence intensities and the Richardson number Ri at the 52- to 113-m layer

Figure 5-8. Annual joint probability distributions of wind speed standard deviation $\sigma_{H}$ and the Richardson number $R i$ at the 52- to 113-m layer

Figure 5-9. Annual probability distributions of wind speed standard deviations $\sigma_{H}$ at a height of 113-, 52-, and 3-m

Figure 5-10. Population characteristic wind speed standard deviation $\sigma_{H}$ as a function of mean wind speed

Figure 5-11. Annual probability distribution of peak gust velocities.

Figure 5-12. Annual probability density of 5-minute layer shear exponents.

Figure 5-13. Cold season (October-March) probability density distributions of the 5-minute layer shear exponents at 3-52 m

Figure 5-14. Cold season probability density distributions of 5-minute layer shear exponents at 52-113 m

Figure 5-15. Warm season (April-September) probability density distributions of 5-minute layer shear exponents at 3-52 m

Figure 5-16. Warm season probability density distributions of 5-minute shear exponents at the 52- to 113-m layer.....

Figure 5-17. Gaussian fits of annual shear exponent probability density distributions

Figure 5-18. Probability density distributions of shear exponent distributions as a function of class and stability $(R i)$....

Figure 5-19. Cumulative hours of mean wind speed at 52-m when the shear exponent is $>0.2$ at the $52-$ to $113-\mathrm{m}$ layer

Figure 5-20. Annual distributions in hours of the shear exponent at the 52- to 113-m layer as a function of the mean wind speed at $52-\mathrm{m}$ 
Figure 5-21. Cold season monthly joint probability distributions of the mean wind direction at 52-m and the gradient Richardson number $(R i)$ at the 52- to 113-m layer, plus monthly frequency distributions of $R i$

Figure 5-22. Warm season monthly joint probability distributions of the mean wind direction at 52-m and the gradient Richardson number $(R i)$ at the 52- to113-m layer, plus monthly frequency distributions of $R i$

Figure 5-23. Cumulative hours of mean wind speed at the 52-to 113-m layer when $R i$ is within critical range $(0<R i<0.25) \ldots$

Figure 5-24. Observed monthly critical stability conditions distributions at the 52- to 113-m layer when the mean wind speed is $>3 \mathrm{~m} / \mathrm{s}$ at $52-\mathrm{m}$

\section{Chapter 6}

Figure 6-1. SODAR-derived 10-minute mean wind vector profiles for 0 to $08 \mathrm{~h}$ LST on 17 June 2002

Figure 6-2. SODAR-derived mean wind speed profile contours for 17 June 2002 - AM Case

Figure 6-3. SODAR-derived mean wind direction profile contours for 17 June 2002 - AM Case

Figure 6-4. SODAR-derived wind speed profiles for 17 June 2002 AM Case.

Figure 6-5. SODAR-derived wind profile evolution for 17 June 2002 - AM Case

Figure 6-6. SODAR-derived wind shear profiles for 17 June 2002 - AM Case . 6-16

Figure 6-7. SODAR-derived averaged wind shear profiles for 17 June 2002

- AM Case.

Figure 6-8. SODAR-derived vertical shear profile contours for

17 June 2002- AM Case.

Figure 6-9. Time histories of 10-minute mean inflow parameters for 17 June 2002 - AM Case.

Figure 6-10. Histogram of 10-minute layer mean shear exponents for 17 June 2002 - AM Case

Figure 6-11. Histogram of 10-minute mean wind speeds for 17 June 2002 - AM Case

Figure 6-12. Probability density distributions of 3-sec wind speed differences for 17 June 2002 - AM Case

Figure 6-13. Time histories of peak coherent turbulent kinetic energy $E_{c o h}$ by height for 17 June 2002 - AM Case

Figure 6-14. SODAR-derived 10-minute mean wind vector profiles for 19 to $24 \mathrm{~h}$ LST on 17 June 2002 
Figure 6-15. SODAR-derived mean wind direction profile contours for 17 June 2002 - PM Case

Figure 6-16. SODAR-derived wind speed profiles for 17 June 2002

- PM Case.

Figure 6-17. SODAR-derived wind speed profiles for 17 June 2002

- PM Case.

Figure 6-18. SODAR-derived averaged wind shear profiles for

17 June 2002 - PM Case

Figure 6-19. SODAR-derived vertical shear profile contours for

17 June 2002- PM Case.

Figure 6-20. Time histories of 10-minute mean inflow parameters for 17 June 2002 - PM Case

Figure 6-21. Time histories of buoyancy length scale $I_{b}$, peak $E_{\text {coh, }}$, turbulence intensities, rotor disk shear exponents and $R i$ for 17 June 2002 - PM Case

Figure 6-22. Time histories of estimated 80-m mean wind speed, rotor layer shear exponents, and layer stability for 17 June 2002 - PM Case.

Figure 6-23. Potential temperature and sonic wind speed vertical profile evolutions for 17 June 2002- PM Case.

Figure 6-24. Time history of shear stress profile for 17 June 2002 - PM Case ... 6-32

Figure 6-25. Logarithmic spectra of $u^{\prime}, v^{\prime}$, and $w^{\prime}, u^{\prime} w^{\prime}, u^{\prime} v^{\prime}$, and $v^{\prime} w^{\prime}$, and probability distribution of $w^{\prime}$ for 20:40 h of 17 June 2002

Figure 6-26. Logarithmic spectra of $u^{\prime}, v^{\prime}$, and $w^{\prime}, u^{\prime} w^{\prime}, u^{\prime} v^{\prime}$, and $v^{\prime} w^{\prime}$, and probability distribution of $w^{\prime}$ for $21: 30 \mathrm{~h}$ of 17 June 2002

Figure 6-27. Logarithmic spectra of $u^{\prime}, v^{\prime}$, and $w^{\prime}, u^{\prime} w^{\prime}, u^{\prime} v^{\prime}$, and $v^{\prime} w^{\prime}$, and probability distribution of $w^{\prime}$ for 22:30 h of 17 June 2002

Figure 6-28. Logarithmic spectra of $u^{\prime}, v^{\prime}$, and $w^{\prime}, u^{\prime} w^{\prime}, u^{\prime} v^{\prime}$, and $v^{\prime} w^{\prime}$, and probability distribution of $w^{\prime}$ for 23:00 h of 17 June 2002

Figure 6-29. Logarithmic spectra of $u^{\prime}, v^{\prime}$, and $w^{\prime}, u^{\prime} w^{\prime}, u^{\prime} v^{\prime}$, and $v^{\prime} w^{\prime}$, and probability distribution of $w^{\prime}$ for 23:40 h of 17 June 2002

Figure 6-30. Time variation of $\sigma_{u}$ and $\sigma_{w}$ for period 21 to $24 \mathrm{~h}$ of 17 June 2002 .. 6-43

Figure 6-31. Time variation of kinematic heat fluxes $\overline{w^{\prime} T^{\prime}}$ for period 21 to $24 \mathrm{~h}$ of 17 June 2002.

Figure 6-32. Cross-correlation coefficients for $u^{\prime} w^{\prime}, u^{\prime} v^{\prime}, v^{\prime} w^{\prime}$, and $w^{\prime} T^{\prime}$ for 17 June 2002 - PM Case

Figure 6-33. Time histories of peak coherent turbulent kinetic energy $E_{c o h}$ by height for 17 June 2002 - PM Case

Figure 6-34. Probability density distributions of 3-s wind speed differences for 17 June 2002 - PM Case 
Figure 6-35. SODAR-derived 10-minute mean wind vector profiles for

0 to $06 \mathrm{~h}$ LST on 23 June 2002

Figure 6-36. SODAR-derived mean wind speed profile contours for

23 June 2002 - AM Case

Figure 6-37. SODAR-derived mean wind direction profile contours for

23 June 2002 - AM Case

Figure 6-38. Long-period wave motions: 23 June 2002 - AM Case ......

Figure 6-39. SODAR-derived wind speed profiles for 23 June 2002 - AM Case

Figure 6-40. SODAR-derived vertical shear profile contours for 23 June 2002- AM Case.

Figure 6-41. Time histories of 10-minute mean inflow parameters for 23 June 2002 - AM Case

Figure 6-42. Time histories of net vertical fluxes of $E$ and $E_{c o h}$ and mean vertical velocity for 23 June 2002 - AM Case.

Figure 6-43. Time histories of peak coherent turbulent kinetic energy

$E_{\text {coh }}$ by height for 23 June 2002 - AM Case

Figure 6-44. Probability density distributions of 3-sec wind speed differences for 23 June 2002 - AM Case.

\section{Chapter 7}

Figure 7-1. Summary of time histories of rotor layer mean $I_{b}$ and hub peak $E_{\text {coh }}$ for the three case studies....

Figure 7-2. Observed variation of peak $E_{c o h}$ as a function of $I_{b}$ and $I_{b} / D$ for the three cases studies

Figure 7-3. Observed variation of mean $I_{b}$ of the of 52- to 113-m layer as a function of $R i$ for the three case studies

Figure 7-4. Observed variation of 52-113 m layer peak $E_{c o h}$ as a function of $R i$ and $N$ for three case studies

Figure 7-5. Sample population cumulative probability distributions of NWTC/

LIST ART turbine root flapwise equivalent load $L_{e q}$, maximum alternating load cycle $L_{p p}$, extreme load $L_{p k}$, and peak zero-mean load $\hat{L}_{p k}$ for unstable and stable flows

Figure 7-6. Cumulative probability distributions of stable NWTC/LIST and three LLLJP case studies hub mean $U_{H}, \sigma_{w}$, and peak $E_{c o h}$ and turbine or rotor layer $R i$ for stable flows $7-10$

Figure 7-7. Probability distributions of NWTC/LIST and three LLLJP case studies $I_{b}$ 
Figure 7-8. Comparison of NWTC/LIST ART turbine root flapwise equivalent load $L_{e q}$, maximum alternating load cycle $L_{p p}$, extreme load $L_{p k}$, and peak zero mean load $\hat{L}_{p k}$ cumulative probability distributions for stable flows with those predicted using LLLJP measured scaling parameters .... 7-12

\section{Chapter 8}

Figure 8-1. Evolution of the $\mathrm{KH}$ billow used as input to the simulation of the WindPACT 1.5MW baseline turbine....

Figure 8-2. Rotor plane distributions of local $R i$, shear stress $u^{\prime} w^{\prime}, E_{c o h}$, and $E$ at $\mathrm{t}=0 \mathrm{~s}$

Figure 8-3. Rotor plane distributions of local $R i$, shear stress $u^{\prime} w$ ', $E_{\text {coh, }}$ and $E$ at $\mathrm{t}=91.5 \mathrm{~s}$

Figure 8-4. Rotor plane distributions of local $R i$, shear stress $u^{\prime} w$ ', $E_{\text {coh, }}$, and $E$ at $\mathrm{t}=166.2 \mathrm{~s}$

Figure 8-5. Rotor plane distributions of local $R i$, shear stress $u^{\prime} w$ ', $E_{\text {coh, }}$ and $E$ at $\mathrm{t}=217.7 \mathrm{~s}$

Figure 8-6. Rotor plane distributions of local $R i$, shear stress $u^{\prime} w$ ', $E_{c o h}$, and $E$ at $\mathrm{t}=271.7 \mathrm{~s}$

Figure 8-7. Time histories of hub-height $U_{H}, u^{\prime}, v^{\prime}$, and $w^{\prime}, u^{\prime} w^{\prime}, u^{\prime} v^{\prime}$, and $v$ 'w' and $E$ and $E_{\text {coh. }}$

Figure 8-8. Time histories of hub-height $U_{H}$, shear stress $U^{\prime} w^{\prime}, w^{\prime} E$, and $w^{\prime} E_{\text {coh }}$ for two periods

Figure 8-9. Time and wavelet analysis of simulated WindPACT 1.5-MW baseline turbine $\mathrm{KH}$ billow turbulence/rotor interaction.

Figure 8-10. Decrease in rotor layer mean vertical shear during lifecycle of $\mathrm{KH}$ billow

Figure 8-11. Comparison of predicted root flapwise bending load spectra for constant speed/shear, SNLWIND-3D background turbulence, IEC NTM/NWP turbulence, and KH billow lifecycle inflows 


\section{List of Tables}

Table 1-1. Multiresolution Analysis Detail Frequency Ranges for CWE300

Turbine

Table 3-1. NWTC/LIST Inflow and Response Summary by Dynamic Stability Category

Table 3-2. Inflow Predictor Linear Correlation $\left(\boldsymbol{R}_{i j}\right)$ Matrix ...................................... 3-8

Table 3-3. Inflow/Response Variable Linear $\left(\boldsymbol{R}_{i j}\right)$ Correlations................................. 3-8

Table 3-4. Inflow Parameter Sensitivity ................................................................. 3-8

Table 3-5. Regression Results Summary …..................................................... 3-9

Table 5-1. Gaussian Shear Distribution Coefficients ........................................... 5-8 


\section{Table of Acronyms}

$\begin{array}{ll}\text { ABL } & \text { atmospheric boundary layer } \\ \text { AGL } & \text { above ground level } \\ \text { ART } & \text { Advanced Research Turbine } \\ \text { ATI } & \text { Applied Technologies, Inc. } \\ \text { CASES-99 } & \text { 1999 Cooperative Atmospheric Surface Exchange Study } \\ \text { COE } & \text { cost of energy } \\ \text { CWE300 } & \text { Carter Wind Eagle 300 kW Turbine } \\ \text { DOE } & \text { U.S. Department of Energy } \\ \text { EPRI } & \text { Electric Power Research Institute } \\ \text { ETL } & \text { Environmental Technology Laboratory } \\ \text { GEC } & \text { Global Energy Concepts, LLC } \\ \text { GEWind } & \text { General Electric Wind Energy } \\ \text { HRDL } & \text { High-resolution Doppler LIDAR } \\ \text { ICAO } & \text { International Civil Aviation Organization } \\ \text { IEC } & \text { International Electrotechnical Commission } \\ \text { IMU } & \text { inertial measurement unit } \\ \text { K.E. } & \text { kinetic energy } \\ \text { KH } & \text { Kelvin-Helmholtz } \\ \text { LES } & \text { large-eddy simulation } \\ \text { LIDAR } & \text { Llght Detection And Ranging } \\ \text { LIST } & \text { Long-Term Inflow and Structural Testing } \\ \text { LLJ } & \text { low-level jet stream } \\ \text { LLLJP } & \text { Lamar Low-Level Jet Project } \\ \text { LWST } & \text { Low Wind Speed Turbine } \\ \text { M-O } & \text { Monin-Obukhov } \\ \text { NCAR } & \text { National Center for Atmospheric Research } \\ \text { NI } & \text { National Instruments } \\ \text { NOAA } & \text { National Oceanic and Atmospheric Administration } \\ \text { NREL } & \text { National Renewable Energy Laboratory } \\ \text { NTM } & \text { Normal Turbulence Model (IEC) } \\ \text { NWP } & \text { Normal Wind Profile (IEC) } \\ \text { NWTC } & \text { National Wind Technology Center } \\ \text { PDF } & \\ \text { RASS } & \text { probability density (mass) function } \\ & \end{array}$


RTD

SERI

SNL

SNLWIND-3D

SODAR

$\mathrm{TI}$

TKE

TVP

WindPACT resistance temperature detector

Solar Energy Research Institute (now NREL)

Sandia National Laboratory

inflow turbulence simulation code

Sound Detection And Ranging

turbulence intensity

turbulent kinetic energy

Turbine Verification Program

Wind Partnership for Advanced Component Technologies Project

\section{Turbine-Related Variables}

$D$

$N_{c y c}$

$L_{p-p}$

$L_{p k}$

$\hat{L}_{p k}$

$L_{e q}$

$\beta_{1}$

$\gamma_{2}$ turbine rotor diameter

number of blade loading cycles

root flapwise maximum alternating load cycle

root flapwise extreme load

root flapwise extreme or peak zero-mean load

root flapwise equivalent load

flapwise high-loading tail distribution exponential shape parameter

edgewise high-loading tail distribution extreme value shape parameter 


\section{Table of Symbols}

\section{Atmospheric Variables}

\begin{tabular}{|c|c|}
\hline$\tau(z)$ & mean shear stress profile, $\tau(z)=\rho_{o}(z)\left[\overline{u^{\prime} w^{\prime}(z)}\right]$ \\
\hline$\sigma_{H}$ & horizontal wind speed standard deviation \\
\hline$\tau_{o}$ & surface shear stress \\
\hline$\theta_{\mathrm{v}}$ & virtual potential temperature ( $\theta$ corrected for moisture) \\
\hline$\sigma_{w}$ & vertical wind speed standard deviation \\
\hline$c_{p}$ & specific heat of air at constant pressure \\
\hline E & turbulent kinetic energy (TKE), $E=1 / 2\left(u^{\prime 2}+v^{\prime 2}+w^{\prime 2}\right)$ \\
\hline$E_{c o h}$ & coherent turbulent kinetic energy, $E_{c o h}=1 / 2 \sqrt{\left(u^{\prime} w^{\prime}\right)^{2}+\left(u^{\prime} v^{\prime}\right)^{2}+\left(v^{\prime} w^{\prime}\right)^{2}}$ \\
\hline$g$ & gravity acceleration \\
\hline$L$ & Monin-Obukhov length, $L=-u_{*}^{3} \theta / \mathrm{kg} Q_{o}$ \\
\hline$l_{b}$ & turbine layer buoyancy scale, $l_{b}=\sigma_{w} / N$ \\
\hline$N$ & Brunt-Väisällä (buoyancy) frequency, $N^{2}=(g / \bar{\theta})(\partial \theta / \partial z)$ \\
\hline$p$ & atmospheric pressure \\
\hline$p_{\alpha}$ & Gaussian probability density distribution \\
\hline$\theta$ & potential temperature, $\theta=T(1000 / p)^{.286}$ \\
\hline$\alpha$ & power law shear exponent, $\alpha=\ln \left(U_{2} / U_{1}\right) / \ln \left(z_{2} / z_{1}\right)$ \\
\hline$Q_{o}$ & surface heat flux \\
\hline$R i$ & gradient Richardson number, $R i=g / \bar{\theta}(\partial \theta / \partial z) /(\partial \bar{U} / \partial z)^{2}$ \\
\hline$R_{i c}$ & critical Richardson number range, $0<R i<0.25$ \\
\hline$S(f)$ & power spectral density \\
\hline$T$ & sensible absolute temperature \\
\hline$t$ & time \\
\hline$T_{*}$ & scaling temperature, $T_{*}=-Q_{o} / u_{*}$ \\
\hline$u$ & streamwise wind component (See Appendix C) \\
\hline$u *$ & friction velocity, $u_{*}=\sqrt{\left|\overline{u^{\prime} w^{\prime}}\right|}$ \\
\hline$u^{\prime}$ & streamwise eddy turbulence component \\
\hline$U_{H}$ & Horizontal wind speed, $U_{H}=\sqrt{u^{2}+v^{2}}$ \\
\hline$v$ & crosswind or lateral wind component \\
\hline$v^{\prime}$ & crosswind or lateral eddy turbulence component \\
\hline$w$ & vertical wind component \\
\hline$w^{\prime}$ & vertical eddy turbulence component \\
\hline$w^{\prime} E$ & vertical flux (transport) of turbulent kinetic energy (TKE) \\
\hline$w^{\prime} E_{c o h}$ & vertical flux (transport) of coherent turbulent kinetic energy \\
\hline$w^{\prime} T^{\prime}$ & kinematic heat flux \\
\hline$x$ & coordinate in the direction of the mean wind \\
\hline$y$ & coordinate perpendicular to the mean wind in the horizontal plane \\
\hline$z$ & height coordinate \\
\hline$\rho$ & air density \\
\hline
\end{tabular}




\section{Glossary of Key Atmospheric Terms}

atmospheric boundary layer The lowest layer of the atmosphere in contact with the earth's surface (Section 2)

atmospheric wave motions

A structure in an atmospheric flow that exhibits at least an approximate periodicity in time or space

turbine layer buoyancy scale A measure of the degree of suppression of vertical atmospheric motions by static stability in the layer between the ground and the maximum elevation of the turbine rotor

coherent turbulence

A turbulent field that exhibits a definite phase relationship in both time and space

Coriolis force

An apparent force on moving particles in a noninertial coordinate system that arises solely from the earth's rotation

dynamic stability

Atmospheric instabilities that develop in part due to air motions (compare with static stability)

gradient Richardson number

The ratio of turbulence generated or damped by buoyancy to that generated by mechanical shear. A negative value is considered unstable and indicates turbulence production by convection in addition to shear. A positive value indicates that shear-generated turbulence is being damped by buoyancy. Small positive values are indicators of the presence of dynamic instability.

gravity wave

A wave disturbance in a stably stratified atmosphere in which buoyancy acts as a restoring force on particles displaced from hydrostatic equilibrium

inertial oscillation

A periodic oscillation in atmospheric flows that is balanced purely by the Coriolis force

inertial period

The period of a single inertial oscillation that varies as a function of latitude

Kelvin-Helmholtz instability A flow structure instability that occurs at the interface of two parallel flows of different velocities and densities that is responsible for the development of Kelvin-Helmholtz waves (also called billows)

Kelvin-Helmholtz wave

A wavelike structure (billow) that develops as a consequence of Kelvin-Helmholtz Instability

Reynolds stress field

Forces imposed on the mean flow by the presence of vortical structures in the wind 
static stability

turbine layer Richardson number

rotor disk Richardson number

vertical shear
A vertical or hydrostatic stability based on the vertical distribution of temperature and the inherent tendencies of air parcels to continue to rise or return to their original height

The value of the gradient Richardson number calculated from near the ground to the rotor disk maximum elevation

The value of the gradient Richardson number calculated between the lowest and highest elevation of the rotor disk

The rate of change of horizontal wind speed with height 


\section{Inflow Coordinate System}

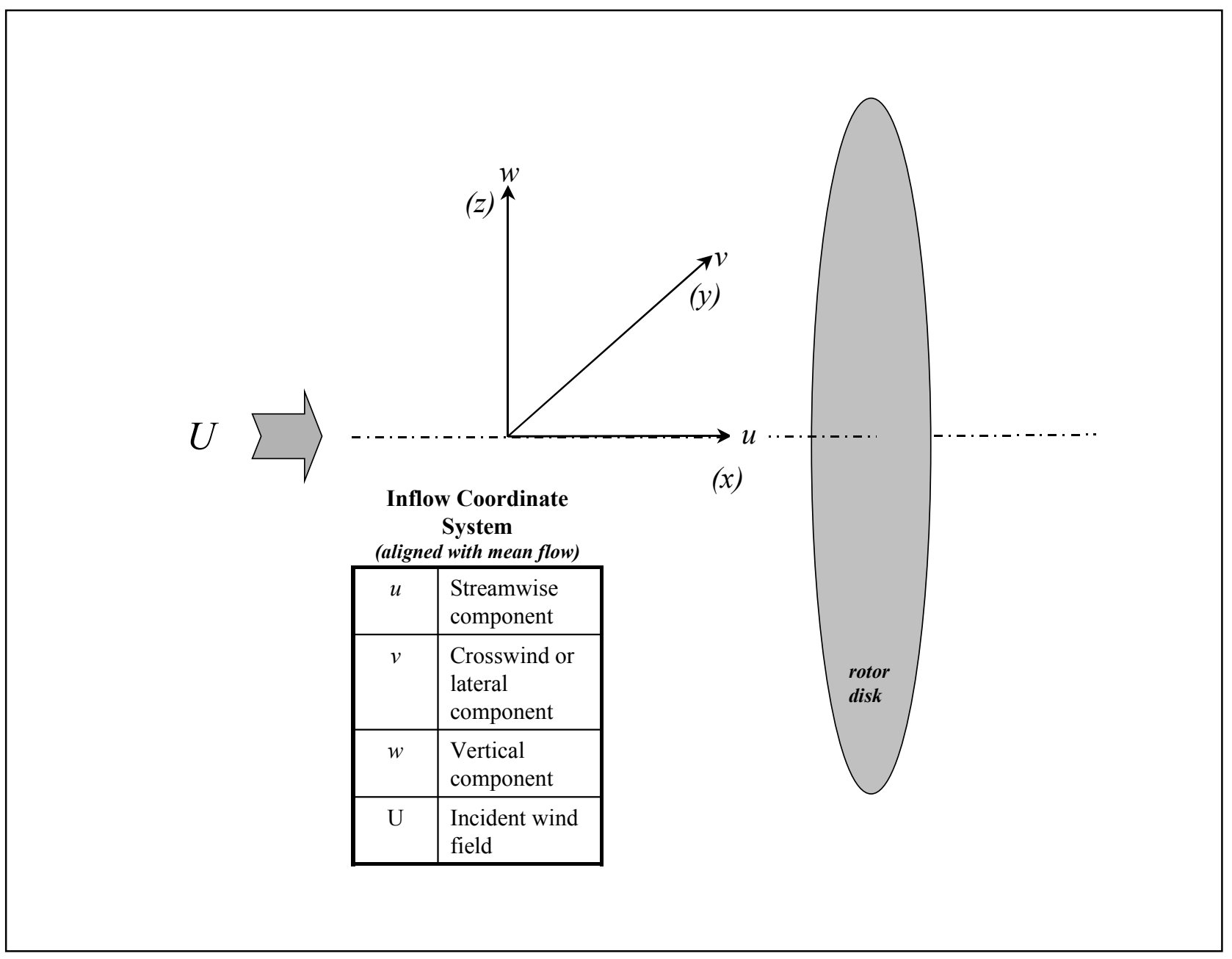




\section{Executive Summary}

\section{Introduction}

This interim report presents the results to date from the Lamar Low-Level Jet Program (LLLJP) that has been established as joint effort among the U.S. Department of Energy (DOE), the National Wind Technology Center (NWTC) of the National Renewable Energy Laboratory (NREL), and General Electric Wind Energy (GE Wind). The purpose of this project is to develop an understanding of the influence of nocturnal low-level jet streams on the inflow turbulence environment and the documenting of any potential operating impacts on current large wind turbines and the Low Wind Speed Turbine (LWST) designs of the future. A year's record of detailed nocturnal turbulence measurements has been collected from NREL instrumentation installed on the GE Wind 120-m tower in southeastern Colorado and supplemented with mean wind profile data collected using an acoustic wind profiler or SODAR (Sound Detection and Ranging). The analyses of measurements taken as part of a previous program conducted at the NWTC have been used to aid in the interpretation of the results of representative case studies of data collected from the GE Wind tower. A detailed computational fluid-dynamics simulation of a turbulent structure common in the nocturnal boundary layer is being used to assess the results of the tower measurements and to provide a basis for the improvement of turbulence simulation codes that are crucial in the turbine design process.

\section{Approach}

The project approach is to integrate an observational element, an analysis element including using data and results from previous NREL measurement campaigns, and a numerical simulation element. The measurement campaign using the GE Wind 120-m tower supplemented with wind profile data collected from the acoustic wind profiler and, later this year, from a high-resolution Doppler LIDAR (Light Detection and Ranging), provides the basic data set. The tower-mounted instruments provide direct detailed measurements of the turbulent environment within a height range that will be occupied by GE Wind $1.5 \mathrm{~S}$ turbines later this year. The acoustic wind profiler provides information regarding the existence of and strength of low-level jets, their height above the ground (and above the tower measurements), and the intensity of the vertical wind shear below them. Later this year, a Doppler LIDAR will be used obtain information about both the vertical and horizontal details of organized turbulent structures found beneath these jets at altitudes that eventually will likely be occupied by LWST turbine rotors.

Data and analysis results from the NREL portion of the Long-Term Inflow and Structures Test (LIST) Program conducted using the NWTC Advanced Research Turbine (ART) has been used as part of this effort. This information is helping to develop criteria that can be employed to assess potential operational impacts on current and future LWST designs that will be operating at the LLLJP and similar sites throughout the Great Plains. These criteria will be eventually used to devise engineering design or operational strategies to mitigate any significant impacts. It is thought that organized turbulent structures are one of the major sources of damaging fatigue loads on wind turbines in the nocturnal boundary layer. A computational fluid dynamics simulation of one particular turbulent structure common in the nocturnal boundary layer has been provided by the National Center for Atmospheric Research (NCAR) and is being used both to 
interpret field measurements and to serve as basis for the improvement of inflow turbulence simulation codes.

\section{Results}

Our initial analyses of 1 year of statistical data and detailed case studies of turbulence data collected from the NREL instrumentation on the GE Wind tower and the acoustic wind profiler and supplemented with results from the NWTC/LIST Program has provided the following information:

- Low-level jet streams (LLJs) occur frequently at the LLLJP Site, particularly during the warm-season months (April-September), which is in agreement with previous studies some of which date back more than 30 years

- The presence of LLJs can significantly modify the vertical shear and turbulence environments within the atmospheric layer occupied by wind turbine rotors

- The presence of LLJs, depending on conditions, can either cause instabilities that produce intense coherent turbulence or only serve to intensify vertical shears that often extend up to as high as $200 \mathrm{~m}$ above ground level

- The stably stratified layers beneath nocturnal LLJs support the development of shear instabilities that can lead to the formation of atmospheric wave motions called KelvinHelmholtz (KH) waves or billows that are significant sources of intense, coherent turbulence within the heights occupied by turbine rotors

- Analysis of the NWTC/LIST data revealed that the largest blade flapwise root loads are associated with a narrow range of vertical dynamic stability of the atmospheric layer occupied by wind turbine rotors

- Efficient predictors of large flapwise root loads include the hub-height mean wind speed, the standard deviation of the vertical wind speed, and the peak of the coherent turbulent kinetic energy, as well as the vertical dynamic stability of the layer in which the turbine rotor resides

- Observed mean turbulence levels between 52- and 113-m (rotor) heights remain below the IEC "A" and "B" specifications

- Shear exponents between 50 and $200 \mathrm{~m}$ frequently exceed the IEC Normal Wind Profile (NWP) specification of 0.2 by factors up to at least 6 and more on a short-term basis

- The highest vertical shears at the LLLJP site occur most often with a hub-height mean wind speed of $8-14 \mathrm{~m} / \mathrm{s}$.

\section{Initial Conclusions}

The presence of nocturnal low-level jets at the LLLJP Site exert a significant influence on both the vertical shear and turbulence environments at the heights of operating wind turbines. In some instances only intense vertical shear exists in the layer below a jet with no appreciable turbulence, while at other times this layer becomes dynamically unstable. This instability results in high levels of coherent turbulence being produced, while at the same reducing the shear. Strong shears can exist as high as at least $200 \mathrm{~m}$ and may have to be addressed in the design of 
the larger LWST rotors. The most significant response of wind turbine rotors to turbulence takes place within a relatively narrow range of vertical dynamic stability. During the period from October 2001 through October 2002 such conditions existed annually a total of 600 hours at the LLLJP Site, with two-thirds of that occurring during the warm season months of April through September. It is unknown how these figures may vary at other Great Plain sites. Finally, it is crucial that the turbulence simulation codes be improved to provide the LWST designers with more realistic inflow conditions so their turbines or turbine components can achieve the desired lifetime goals in these operating environments.

\section{Future Work}

In the next 18 to 24 months, we expect to accomplish (providing sufficient resources are available) the following:

- Collaborate with the National Oceanic and Atmospheric Administration's (NOAA) Environmental Technology Laboratory (ETL) using their high-resolution Doppler LIDAR to accomplish these goals:

- Assess the ability of a Doppler LIDAR for use in long-term wind resource assessment up to heights of $200 \mathrm{~m}$ above ground level

- Determine the usefulness of Doppler LIDAR for characterizing turbulence events and particularly those events that are likely to present some long- or short-term risk to wind turbine operations

- Assess the usefulness of using a forward-looking Doppler LIDAR to detect coherent turbulent structures in turbine inflows

- Investigate the horizontal distribution and dimensions of turbulence or wave packets in the nocturnal stable boundary layer

- Compare the usefulness of direct measurements in comparison with remote sensing via LIDAR and SODAR for support of wind farm operations

- Complete the data reduction of the more than 30,000 10-minute records of detailed turbulence information collected from the GE Wind 120-m tower from March 2002 through March 2003

- Use the detailed tower measurements, the SODAR observations (when available), and the NOAA LIDAR results to aid in improving the SNLWIND-3D inflow turbulence simulation code to better simulate conditions associated with shear-flow instabilities (such as KH billows) frequently encountered in the nocturnal stable boundary layer

- Investigate obtaining vertical temperature profiles using radio-acoustic remote sensing (RASS) in conjunction with SODAR wind measurements to obtain real-time estimates of vertical stability and shear profiles near wind farms that may be used for operational purposes (i.e., control of loads from encounters with coherent turbulence). 


\section{Introduction}

\subsection{Background}

The greatest wind resource in the United States is found in the western Great Plains. This region is characterized by often having the most energetic winds during the nighttime hours, and that energy sharply increases with height. The same geographic region experiences a relative high frequency of nocturnal low-level jet streams. Often the intense wind shears occurring in the stably stratified flows below the height of the jet stream velocity maximums are responsible for the formation of coherent turbulent motions associated with shear-induced instability. Such conditions often generate intense and highly organized or coherent turbulent motions that, when ingested by a wind-turbine rotor, can be responsible for inducing large, damaging loading events, as well as causing the turbines to shut down from excessive vibration. In order to take advantage of the "better" resource, turbine rotors are being installed at increasing heights and into more frequent contact with coherent wave motions, thus increasing the probability for adverse impacts on operational reliability and lifetime. Little is known about the severity and frequency of such motions at the scale of wind-turbine rotors because regular observations are unavailable at the heights involved. Similarly, there is little or no knowledge about the spatial variation of the low-level jets and accompanying wave-motion activity. To obtain detailed information about the turbulence environment in which large turbine rotors will be installed, this project utilizes direct measurements from a tall tower. The measurements are supported with data collected from both an acoustic wind profiler (SODAR) and a LIDAR (Light Detection and Ranging) highresolution Doppler laser. Such a measurement campaign is a first step in establishing the frequency and severity of potentially damaging coherent turbulent motions in the nocturnal boundary layer at current and expected turbine hub heights where nocturnal low-level jets are expected to occur relatively often. This information will also be used to improve the operational reliability and lifetime of the ever-larger turbines expected over the next few years. This information will provide the following: (1) an extensive database of operational shear and turbulence conditions that can be expected, and (2) information that can be used to incorporate coherent, turbulent events into existing inflow turbulence simulation codes. Both will provide the turbine designer crucial information in the development of Low Wind Speed Turbine (LWST) designs.

\subsection{Turbulence-induced Turbine Structural Response - Previous Studies}

For more than a decade, the Department of Energy (DOE), through its wind research programs at the National Renewable Energy Laboratory (NREL) and the Sandia National Laboratory (SNL), has supported an effort to observe, identity, and quantify the turbulent inflow conditions associated with large and often damaging structural loading events experienced by operating wind turbines. From measurement campaigns conducted in San Gorgonio Pass, California; near Bushland, Texas; and at the National Wind Technology Center (NWTC) in Colorado using a range of turbine sizes and operating environments, the physical factors surrounding turbulence-turbine interaction have gradually emerged. Three turbines designs have been primarily used for this research: the Micon 65/13, the Carter Wind Energy CWE300 Wind Eagle, and the NWTC Advanced Research Turbine (ART). 


\subsubsection{San Gorgonio Micon 65/13}

The Micon $65 / 13$ is a $65-\mathrm{kW}$, three-bladed, upwind turbine with a nominal $15-\mathrm{m}$ diameter rotor with a hub height of 23 meters. It uses stall control for modulating peak power. In 1989-90, we monitored an adjacent pair of these machines at Row 37 of a 41-row wind farm in San Gorgonio Pass, California. One turbine was fitted with the NREL (formerly the Solar Energy Research Institute [SERI]) 7.9-m Thin Airfoil Blades, while the other used an original-design AeroStar blade set. Detailed measurements of the turbulent inflow and a wide range of structural parameters were acquired over a wide range of atmospheric conditions. Currently, SNL is using a modified version of this turbine as part of the LongTerm Inflow and Structural Testing (LIST) Program at a Great Plains Site near Bushland, Texas [1]. Other than the modified design of the single turbine, this experiment is using an array of five sonic anemometers upwind of the turbine, whereas the earlier California experiment only had a hub-height instrument available.

In our analysis of the Micon dataset, we found that the number of cycles contained in the high-loading tail (recurring frequencies of $100 \mathrm{cycles} / \mathrm{h}$ or less) of spectral distributions scales with the hub-height or local values of the inflow mean shearing stress $u_{*}$ and the dynamic stability of the vertical layer in which the turbine resides. The mean shearing stress or friction velocity is defined as

$$
u_{*}=\sqrt{\overline{\left|u^{\prime} w^{\prime}\right|}}
$$

where $u$ ' and $w$ ' are the streamwise and vertical zero-mean turbulent wind components of a co-ordinate system aligned in the direction of the local mean shear. In fact high correlations were found with not only $\overline{u^{\prime} w^{\prime}}$ but the two remaining mean Reynolds stress components $\overline{u^{\prime} v^{\prime}}$ and $\overline{v^{\prime} w^{\prime}}$ as well. All three co-variances represent the off-diagonal terms of the Reynolds stress tensor where and $v^{\prime}$ is the lateral or crosswind turbulent (zero-mean) wind component. The dynamic stability of the turbine layer is expressed by the gradient Richardson number defined as

$$
R i=(g / \bar{\theta})\left[\left(\frac{\overline{\partial \theta}}{\partial z}\right) /\left(\frac{\partial U}{\partial z}\right)^{2}\right]
$$

where in this equation, $U=\sqrt{\left(u^{2}+v^{2}\right)}, g$ is the gravitational acceleration, $z$ is the height (m), $\bar{\theta}$ is the layer mean potential temperature given by

$$
\theta(z)=T(z)\left[\frac{1000}{p(z)}\right]^{0.286}
$$

and $T(z)$ and $p(z)$ are the absolute temperature $(\mathrm{K})$ and barometric pressure $(\mathrm{hPa})$ at height $z$. A negative value of $R i$ represents unstable or convective conditions, a value of zero represents neutral, and positive values signify a stable flow. The $R i$ represents the ratio of turbulence generation by buoyancy to shear. The buoyancy term, $g / \bar{\theta}(\overline{\partial \theta / \partial z})$, contributes 
to turbulence generation when it is negative (convective) and acts as a damper when it is positive (stable).

In a well-mixed, homogeneous atmospheric boundary layer over flat terrain, only $\overline{u^{\prime} w^{\prime}}$ is generally non-zero because it represents the vertical flux (normally downward transport) of horizontal momentum. However, when turbulence contains motions that are both spatially and temporally organized (i.e., coherent), all three of the mean Reynolds stress components can be finite. Instantaneously, all three components can take on large positive or negative values. The passage of an organized patch of turbulence can be identified by using an anemometer capable of rapid, simultaneous measurement of multi-axis wind components (e.g., a sonic anemometer). Coherent turbulent structures can contain large velocity shears over small distances, as well as significant local vorticity. Moderate- to high-speed flows that are just weakly dynamically stable ( $R i$ small but positive) often contain small, energetic turbulent structures.

Figure 1-1 schematically demonstrates the relationship between the slope of the high-loading tail of an alternating load spectrum and $u_{*}$ and $R i$. It was found that the change in high-tail loading as a function of these parameters could be described by an exponential distribution whose shape parameter (slope) $\beta_{1}$ was given by

$$
N_{c y c}=\beta_{0} e^{-\beta_{1} L_{p-p}}
$$

where $\beta_{0}$ is a constant, and $L_{p-p}$ is the value of the peak-to-peak load cycle [2]. We found that the exponential tail distribution could be reasonably applied to all of the structural response variables that were correlated with the exception of the blade root edge bending moment. In this instance, it was found that the width or shape parameter $\gamma_{2}$ of the extreme value distribution

$$
N_{c y c}=\gamma_{0} \exp \left\{-\exp \left[-\left(\frac{L_{p-p} \gamma_{1}}{\gamma_{2}}\right)\right]-\left(\frac{L_{p-p}-\gamma_{1}}{\gamma_{2}}\right)+1\right\}
$$

provided a better correlation with the rate of decay of the spectrum high-loading tail. For example, more than $85 \%$ of the variation in $\beta_{1}$ and $\gamma_{2}$ was explained for root flapwise and edgewise bending loads, respectively, for both turbines. We also found that there was often significant correlation with $\beta_{1}$ and $\overline{u^{\prime} v^{\prime}}$, and $\overline{v^{\prime} w^{\prime}}$ for other response variables, such as the yaw drive torque, tower in-plane thrust, and with $\gamma_{2}$ for the root edgewise bending load, which signifies the sensitivity of the load responses to coherent turbulence.

The overall correlation with the excitation variables hub local friction velocity $u_{*}$ and the dynamic stability of the turbine layer and the root bending moment response variables $\beta_{1}$ and $\gamma_{2}$ for each of the turbines is shown in Figure 1-2. The relationships between the dynamic stability (abscissa axis) and the hub $u_{*}$ (left ordinate axis) values are plotted against $\beta_{1}$ and $\gamma_{2}$ (referenced to the right ordinate axis). The stability measure used $(z / L)$ is related to $R i$. For unstable (convective) conditions $z / L=R i$ and for stable flows it is greater than $R i$. Here 
the value of $u_{*}$ peaks when the flow is just slightly (weakly) stable at an equivalent $R i=$ +0.019 indicating the presence of significant vertical momentum transport and coherent turbulence. Here also reside the lowest values of $\beta_{1}$ and highest values of $\gamma_{2}$ signifying a shallow slope in the high loading tail and the occurrence of a greater number of large amplitude cycles that usually translates into increased fatigue damage (Figure 1-1).

\subsubsection{NWTC CWE300 Wind Eagle}

Whereas the Micon 65/13 is a relatively rigid or stiff design, the Carter CWE300 turbine is just the opposite. The turbine tested during May and early June 1998 was a pre-prototype design. It is a downwind, very flexible, two-bladed, stall-regulated turbine with a rotor diameter of $29.3 \mathrm{~m}$ and a hub height of $49.8 \mathrm{~m}$ and has a nominal power rating of $300 \mathrm{~kW}$ at $16 \mathrm{~m} / \mathrm{s}$ in the NWTC environment. The measurement program was limited, producing only 18 data runs over a reasonably diverse range of inflow conditions [3]. The data collected during this campaign provided comparison with the more rigid, Micon 65/13. While not installed within a multi-row wind farm, the CWE300 experienced periods of very energetic turbulence at relatively high wind speeds that is characteristic of the NWTC. Data from this program will be discussed in conjunction with our discussion of the role of coherent inflow in turbine structural response in Section 1.2.4.

\subsubsection{NWTC/LIST Program}

The Long-Term Inflow and Structural Testing Program (LIST) was established to obtain the necessary measurements to provide detailed information to:

- Compare measured spatial inflow properties important to wind turbine operations with those simulated by the SNLWIND-3D turbulence code [4], and establish any systematic differences as a function of atmospheric boundary-layer conditions

- Establish whether or not it is possible to modify the present formulation of the SNLWIND-3D code to bring the simulated properties into closer agreement with observations obtained by LIST field measurements up to heights of $60 \mathrm{~m}$ or more above the ground

- Establish sensitivities of turbine aeroelastic and structural response to a range of turbulence scaling parameters and compare with other turbines and operating environments.

During the period between late October 2000 and May 2001, detailed inflow turbulence inflow was collected from an upwind planar array of five sonic anemometers and other instruments time-synchronized with a range of aeroelastic and structural measurements on the NWTC ART turbine. The ART is a $600-\mathrm{kW}$ machine with a pitch regulated, teetered, two-bladed, upwind rotor $43 \mathrm{~m}$ in diameter and a hub height of $37 \mathrm{~m}$. A total of more than 1500 10-minute records are available during which the turbine operated continuously and the mean wind hub-height wind direction was within $\pm 45^{\circ}$ of the perpendicular to the planar array. Slightly more than 700010 -minute records of inflow information alone covering a wide range of conditions are also available [5].

Initial results have shown that turbulence-scaling relationships employed with the present turbulence simulation codes do not adequately generate the flow conditions seen in the 
stable, nocturnal atmospheric boundary layer above about $30 \mathrm{~m}(\sim 100 \mathrm{ft})$. Even in unstable, daytime conditions at higher elevations, the upper limits to which these scaling relationships can be applied acceptably for wind turbine operations is presently not known.

\subsubsection{Role of Coherent Inflow Turbulence in Inducing Turbine Aeroelastic and Structural Response}

In order to better understand the role of coherent or organized turbulence on the aeroelastic and structural response of wind turbines, we applied wavelet analysis in [6] to both the inflow turbulence (described by the Reynolds stress components measured at hub height) and turbine key rotor response parameter (root flapwise loads). Wavelet analysis has the advantage of being able to study more closely the time-varying spectral decomposition of short-period loading events associated with the interaction of coherent turbulence with a moving rotor blade. We used the facilities available to us to dissect important frequency domain information from loading events that typically last only the order of tens of seconds or less. From those results, we have examined the nature of the turbulence excitation and the forced responses of the turbine dynamics that result in large load excursions and potentially significant fatigue damage.

Our analysis [6] of both rigid (Micon 65/13) and flexible (CWE300) turbines showed that a coherent or broadband structural response occurs in a rotor ingesting a patch of turbulence. By broadband, as used here, we are describing a range of excitation frequencies that cover the dominant modal or natural frequencies associated with the turbine structure. In more general usage in the theory of vibration, the term broadband refers to excitation that covers a much wider frequency range and often is described by either a uniform or Gaussian process in which the phase spectrum is random as well as the amplitude. In keeping with the nomenclature of the theory of vibration or vibration analysis, our definition of a narrowband process includes a range of exciting frequencies equivalent to inflow turbulent eddy wavelengths that are limited to the corresponding band of the turbine resonant frequencies. In general, we found that events exhibiting narrowband characteristics are imbedded within the much more random flow, which can be truly thought of as a broadband excitation. Thus, the coherent turbulent structures (as identified by the Reynolds stress field) can be thought of as being superimposed on top of a more random background turbulence field.

We found that under coherent turbulence excitation, load peaks occur when the various modal frequencies occur in phase or in superposition. The first and second symmetric and asymmetric rotor modes appeared to be most susceptible to such excitation. It was also found that the constituent turbulent eddies of a coherent inflow structure, whose equivalent space scales are less than a quarter of a rotor diameter, play a major role in developing peak load responses.

An example of this process using the CWE300 turbine is presented in Figures 1-3 and 1-4. Here a 20 -s loading event is analyzed during a period in which the background conditions consisted of a hub-height mean wind speed of $18.33 \mathrm{~m} / \mathrm{s}$ and a turbine layer $R i$ of +0.031 (weakly stable). The top panel of Figure 1-3 plots the hub-height time series of the horizontal wind speed and in the panel below the three Reynolds stress components $\left[u^{\prime} w^{\prime}(t)\right.$, $u^{\prime} v^{\prime}(t)$, and $\left.v^{\prime} w^{\prime}(t)\right]$. The zero-mean root flapwise bending load time series is plotted in the third panel from the top. The two lowest panels present the analysis of the flapwise load 
signal using the continuous and discrete wavelet transforms (multiresolution analysis), respectively. While no distinct wind gusts were present, the period between about 2 and $11 \mathrm{~s}$ contains a coherent turbulent structure as revealed by the Reynolds stress components. It is during this time segment that a large envelope of flapwise load response is noted. The continuous transform panel presents the frequency distribution of dynamic stress energy in terms of color. The highest levels of stress are shown in the darkest red color. The multiresolution analysis in the panel below depicts the decomposition of the load into seven variable frequency bands whose widths vary by a factor of 2 . The frequency ranges associated with each of the bands is listed in Table 1-1 along with the associated turbine modal frequencies contained with the band. For example, the rotor first symmetric flapwise bending mode is contained in lowest frequency detail band B7. Figure 1-4 displays the time-frequency decomposition of the three Reynolds stress components along with the flapwise load signal using the continuous transform. An examination shows that turbulent energy existed in bands occupied by one or more of the turbine resonances at sometime during the period of the disturbance.

Figures 1-5 and 1-6 present similar analyses taken from the results of the simulation of CWE300 turbine by Wright and Kelley [7] using the same background inflow conditions. Again the there is no sharp edge gust, but the region of higher wind speeds between 6 and 12 s contains a coherent structure as evidenced by the Reynolds stress components. The flapwise load response envelope is contained wholly within the period of the coherent disturbance though the beginnings of another, smaller, response envelope is apparent about $18 \mathrm{~s}$ into the record when another, weaker, coherent structure appears. In the simulation, the stress energy is concentrated in the region of the disturbances. This is a consequence of the nature of the turbulent inflow simulation (the SNLWIND-3D code) that tends to spread the coherent disturbance over the entire rotor disk instead of in concentrated areas within the disk, which apparently happens in the real inflow. The effects of this can been seen in Figure 1-6. It does demonstrate that a coherent structure the size of the turbine rotor is capable of inducing strong responses. Also demonstrated is the fact that an intense turbulent structure containing an equivalent narrowband range of energy elicits a structural response in which modal energy is summed by superposition into a large loading event.

The conclusion reached is that it is important to identify processes in the atmospheric boundary layer that can spawn coherent turbulent structures, such as these, in terms of not only severity but frequency as well. It is also important that the turbulence simulation codes used faithfully reduce the range of disturbances encountered in those processes in order to provide the proper range of conditions to be seen by wind turbine designs.

\subsubsection{Atmospheric Sources of Coherent Structures Affecting Wind Turbines}

Organized turbulent motions exist at all scales within the Earth's atmosphere. We are, however, most concerned with those structures that have dimensions near turbine-rotor diameters currently in use and those that are projected for the future. Because wind turbines now and in the future will occupy the lower elevations of the atmospheric boundary layer, we will limit our search to this region.

Measurements of turbulence and turbine response in the California wind farm discussed previously indicated that the highest fatigue loads occurred in the late evening hours peaking 
between 20 and $22 \mathrm{~h}$ local time [8]. The diurnal variation in root flapwise equivalent $L_{e q}$ or fatigue equivalent loads is shown in Figure 1-7. This parameter is defined as

$$
L_{e q}=\left(\frac{\sum n_{i} L_{i}^{m}}{N_{e q}}\right)^{1 / m}
$$

where $n_{i}$ is the number of cycles in the $I^{\text {th }}$ load range, $L_{i}$ is the maximum value of each level in a bin, $N_{e q}$ is the equivalent number of constant-amplitude cycles, and $m$ is the slope of the material S-N curve. For these measurements, $N_{e q}$ was set to 1200 half-cycles, and $m$ was 12 (characteristic of some composite materials used in wind turbine rotors). On these plots, Rotor 1 was the machine with the NREL Thin Airfoil Blades, and Rotor 2 was the turbine with the original AeroStar blades.

The relationships between the turbine-layer $R i$, hub-height $u_{*}$, and $L_{e q}$ are shown in Figure 18. As was the case with Figure 1-1, the highest values of $L_{e q}$ occur under weakly stable conditions with $R i$ in the vicinity of +0.02 and corresponding values of $u_{*}$ above $1.6 \mathrm{~m} / \mathrm{s}$.

Figure 1-9 also demonstrates that $L_{e q}$ peaks larger than $20 \mathrm{kNm}$ occur between 18 and $24 \mathrm{~h}$ in the nocturnal boundary layer. The diurnal variation of $R i$ and $u_{*}$ is plotted in Figure 1-10, which clearly shows a very small variation in $R i$ between those hours with values staying between 0 and +0.05 . Thus, the turbulent structure present during this period is of paramount importance. The high values of $u_{*}\left(\sqrt{\overline{u^{\prime} w^{\prime}}}\right)$ indicate not only the presence of coherent turbulence, but also a strong downward transport of momentum $\left(\overline{u^{\prime} w^{\prime}}\right.$ is always negative under such conditions). The corresponding occurrence of large load peaks suggests the processes discussed above and demonstrated with the wavelet decompositions are likely at work.

Kelley et al. [9] simulated a full diurnal period of 10-minute operations of the Micon 65/13 turbine using nominal values of boundary conditions to scale the inflow turbulence generated by the SNLWIND-3D turbulence code. Kelley and Sutherland [10] calculated the fatigue damage from the simulated loads and compared them with 2.3 hours of measured data collected under similar environmental conditions. They found that the fatigue damage calculated from the 24 hours of simulated loads was considerably less than that from the measured loads acquired over a much shorter period of time. They concluded that the simulations lacked the large-amplitude load cycles that were present in the observed data. Just why that was the case was not known at the time, but what we expect to be the reason will be addressed later in this report.

While it is likely coherent turbulent conditions of the proper scale can be expected to occur occasionally during daylight hours, particularly during periods associated with large-scale disturbances such as gust fronts (these have been observed at the GE Wind Tower in southeast Colorado), it is likely that conditions associated with the nocturnal boundary layer (i.e., weakly stable flows and vertical wind shear) will potentially have a greater impact on wind turbine operations simply because of the much higher frequency of occurrence. One possible example of this is the comparison of the diurnal wind shear and corresponding number of hours observed when the turbines were offline because of fault conditions (shutdown by the control system because of out-of-tolerance events) at a wind farm in Big 
Spring, Texas, monitored under the Department of Energy - Electric Power Research Institute (DOE-EPRI) Wind Turbine Verification Program (TVP). The diurnal variation of these parameters based on a 1-year record analyzed by Smith et al. [11] is presented in Figure 1-11. While the fault time was found to have the highest frequency during the early morning hours when high values of shear were present, not enough information is available to make a definitive connection. Also, the availability of operating personnel to correct faults before to midnight may also influence the curves of Figure 1-11. No measurements of atmospheric stability were available, so no conclusions can be drawn there. An experience at a wind farm in the northern Great Plains was similar. Here a significant increase $(\sim 85 \%)$ in the number of excessive vibration faults was noted during the month of August, whereas in May, June, and July only a relatively few cases were reported by the site monitoring system. Most of the faults in August occurred between 18 and $04 \mathrm{~h}$ local time, whereas during the previous 3 months the time of occurrence was more or less uniformly distributed over 24 hours.

Given these indicators, clearly we must look at atmospheric processes that are capable of producing frequent coherent turbulent structures in nocturnal sheared flows at the heights that the LWST designs will be expected to operate. We know that the dynamic stability expressed by $R i$ usually is positive (stable) at night; the evidence from [11] that large wind shears are common across the Great Plains suggests that weakly stable conditions may frequently exist. We also know that low-level jet streams are common, particularly in the warmer months, which can serve to increase the wind shear. This topic will be addressed later in this report. Eggers et al. [12] have performed an exploratory study of the impacts of high shear alone and found that high values of shear can substantially increase out-of-plane bending loads and others related to them. They believe control measures may help relieve them. They also point out that a body of reliable test data on high wind shear and turbulence is needed in order to improve the modeling of such effects.

\subsection{Objectives}

The primary objectives of the Lamar Low-Level Jet Project (LLLJP) include the following:

1. Obtain detailed wind fields turbulence measurements using tower-based measurements, SODAR-derived wind profiles, and high-resolution Doppler LIDAR at heights in the nocturnal boundary that is currently, or expected to be, occupied by wind turbine rotors, in order to establish the frequency and severity of coherent turbulent motions at an identified wind resource area where low-level jet streams are expected to occur relatively often.

2. Utilize the information collected to improve the capabilities of current turbulence simulation codes such as SNLWIND-3D, in order to produce the necessary threedimensional flow structures needed to induce the large amplitude structural loads seen on operating wind turbines.

\subsection{Scope}

This interim report discusses our initial progress in identifying the atmospheric conditions responsible for increased fatigue damage on wind turbine components and applying this knowledge for improving turbulence simulation capabilities. We do that by presenting 
statistical summaries of important meteorological variables collected over a 1-year period at a representative Great Plains wind energy resource area, performing a detailed analysis of three representative case studies, and by incorporating the simulation of an atmospheric coherent flow structure common in the nocturnal boundary layer and often associated with the presence of low-level jet streams.

Table 1-1. Multiresolution Analysis Detail Frequency Ranges for CWE300 Turbine

\begin{tabular}{|c|c|c|}
\hline $\begin{array}{l}\text { Detail } \\
\text { Band }\end{array}$ & $\begin{array}{l}\text { Cyclic } \\
\text { Frequency } \\
\text { Range (Hz) }\end{array}$ & Characteristic Modal Responses \\
\hline B1 & $15.0-30.0$ & Rotor $1^{\text {st }} / 2^{\text {nd }}$ torsion bending; $3^{\text {rd }}$ symmetric lag bending \\
\hline B2 & $7.5-15.0$ & Flexbeam $2^{\text {nd }}$ flap bending; blade shell $4^{\text {th }}$ flap bending \\
\hline B3 & $3.75-7.5$ & $\begin{array}{l}\text { Rotor } 3^{\text {rd }} \text { symmetric and asymmetric bending; } 2^{\text {nd }} \text { asymmetric lag } \\
\text { bending; blade shell } 2^{\text {nd }} \text { flap bending }\end{array}$ \\
\hline B4 & $1.875-3.75$ & Rotor $2^{\text {nd }}$ asymmetric flap bending; blade shell $1^{\text {st }}$ flap bending \\
\hline B5 & $0.938-1.875$ & $\begin{array}{l}\text { Rotor } 1^{\text {st }} \text { asymmetric flap bending; rotor } 2^{\text {nd }} \text { symmetric flap bending; } \\
\text { tower } 1^{\text {st }} / 2^{\text {nd }} \text { fore/aft and side/side bending; drive train } 1^{\text {st }} \text { bending; } \\
\text { blade shell } 1^{\text {st }} \text { flap bending }\end{array}$ \\
\hline B6 & $0.469-0.938$ & Rotor $1^{\text {st }}$ asymmetric lag bending; $1-\mathrm{P}$ \\
\hline B7 & $0.234-0.469$ & Rotor $1^{\text {st }}$ symmetric flap bending \\
\hline
\end{tabular}




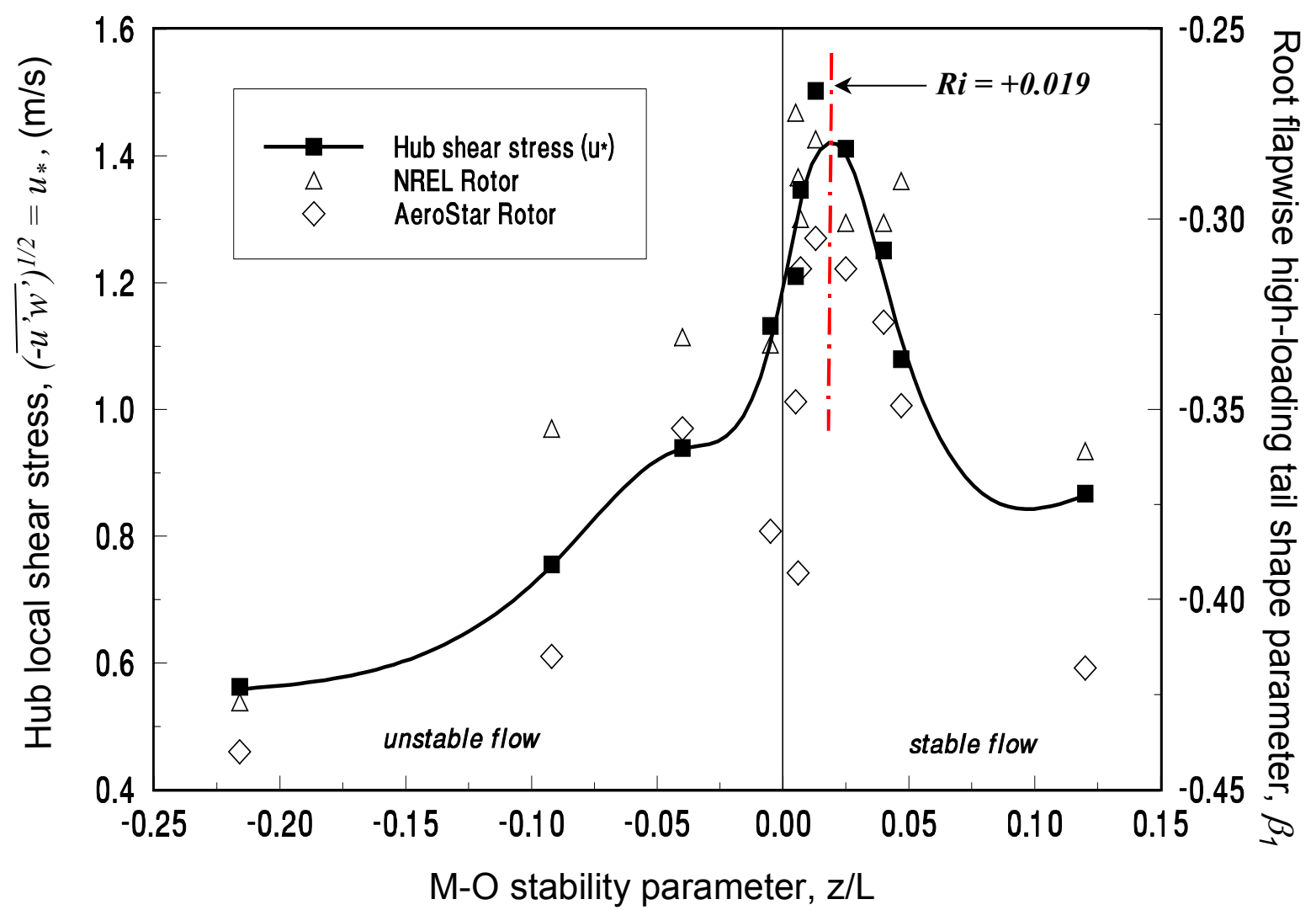

Figure 1-1. Variation of hub-height local shear stress $\left(u_{*}\right)$ and root flapwise high-loading tail shape parameter for Micon 65/13 turbines in San Gorgonio Pass wind farm 


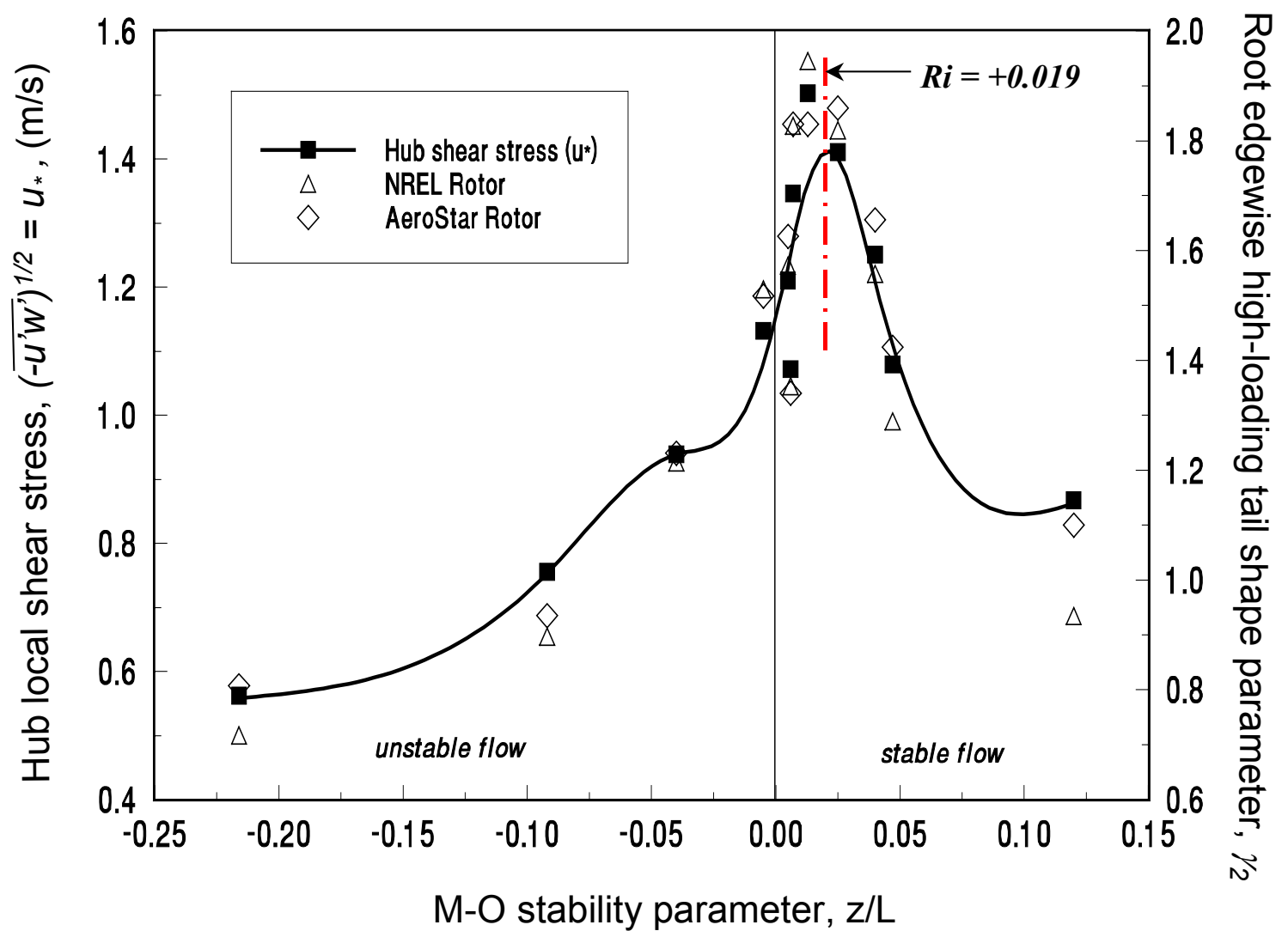

Figure 1-2. Variation of hub-height local shear stress $\left(u_{*}\right)$ and root edgewise high-loading tail shape parameter for Micon 65/13 turbines in San Gorgonio Pass wind farm 


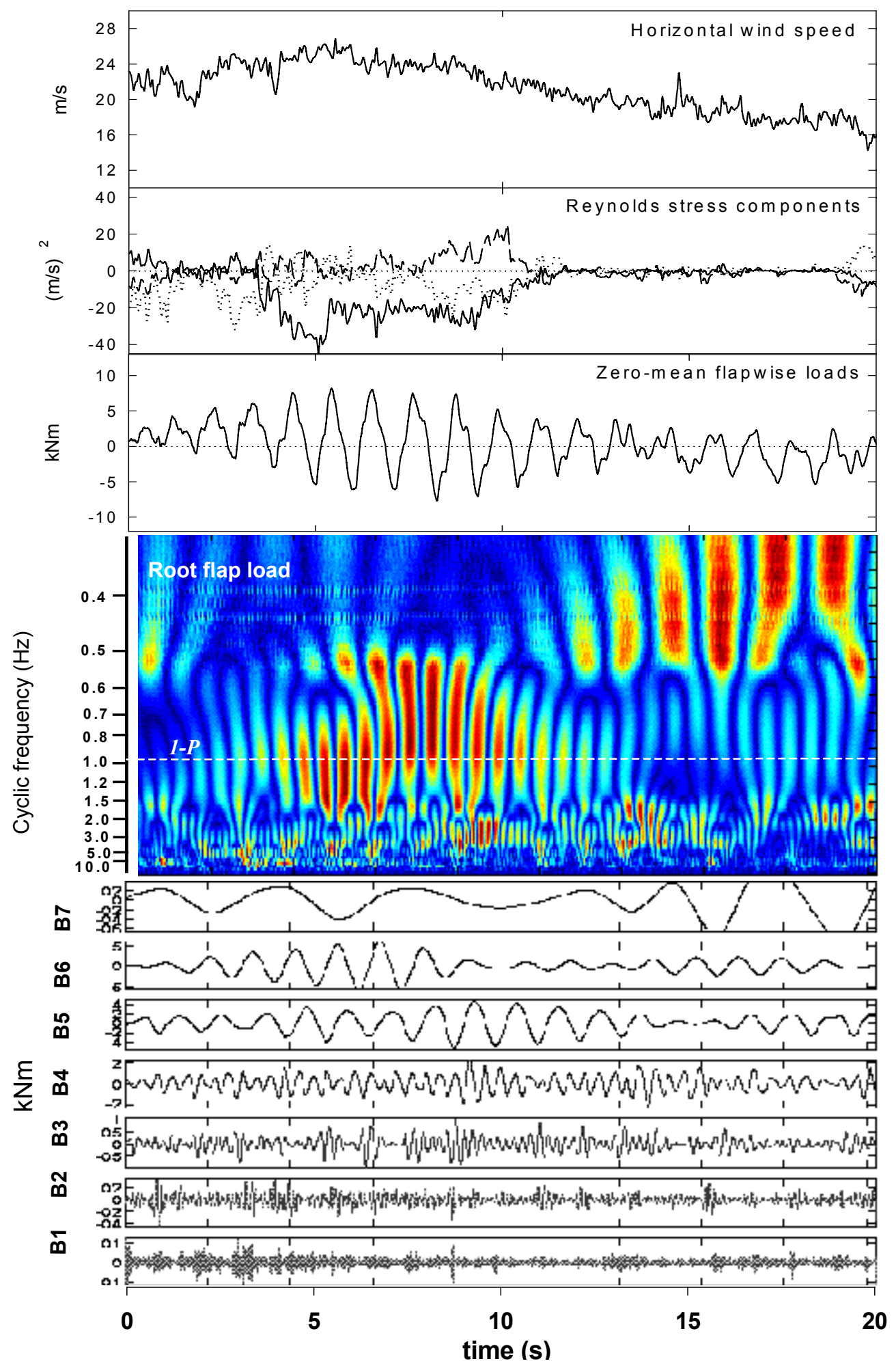

Figure 1-3. Time and wavelet analysis of CWE300 turbine turbulence response 

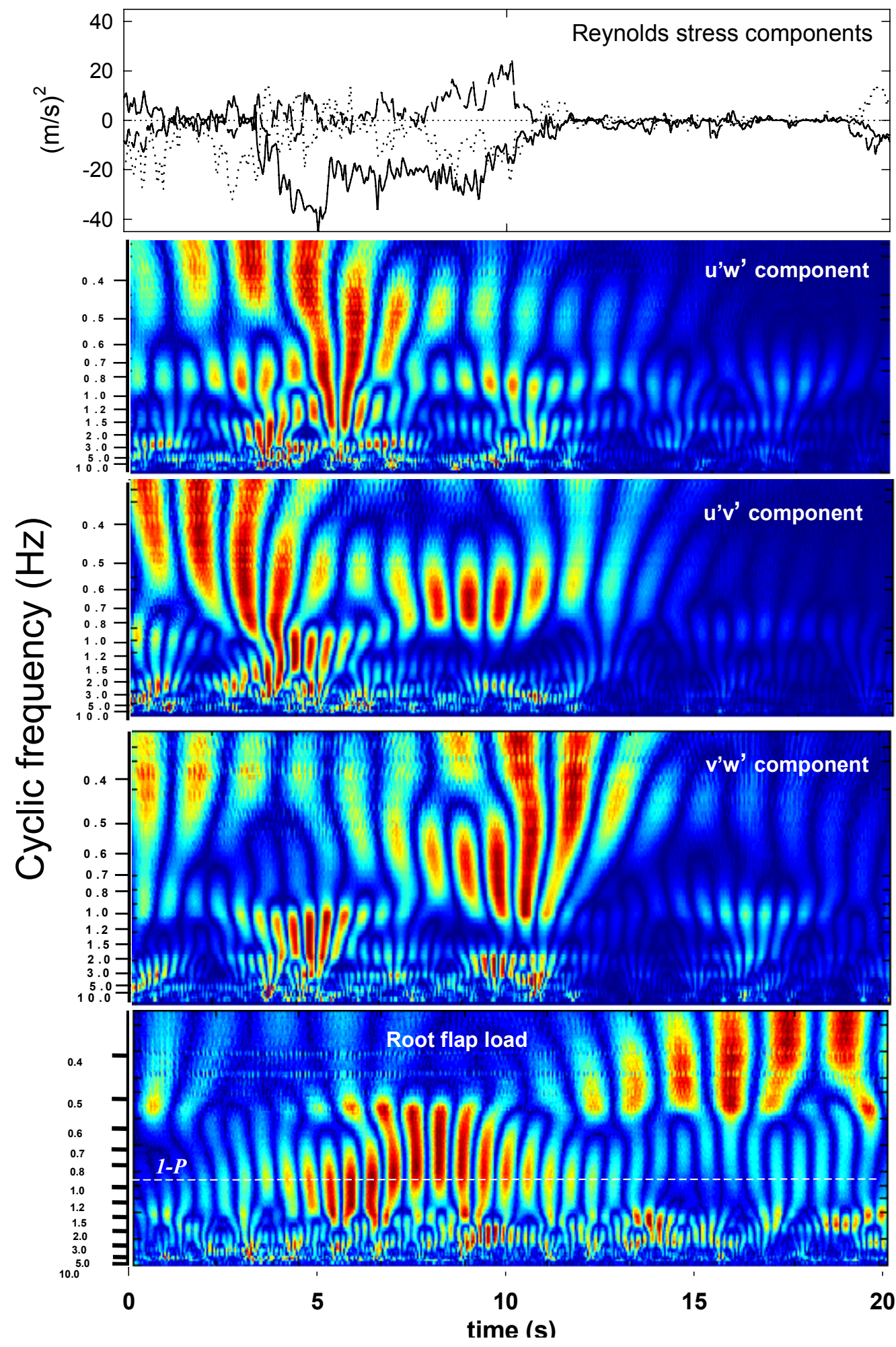

Figure 1-4. Continuous wavelet transform analysis of turbulence excitation for CWE300 turbine 


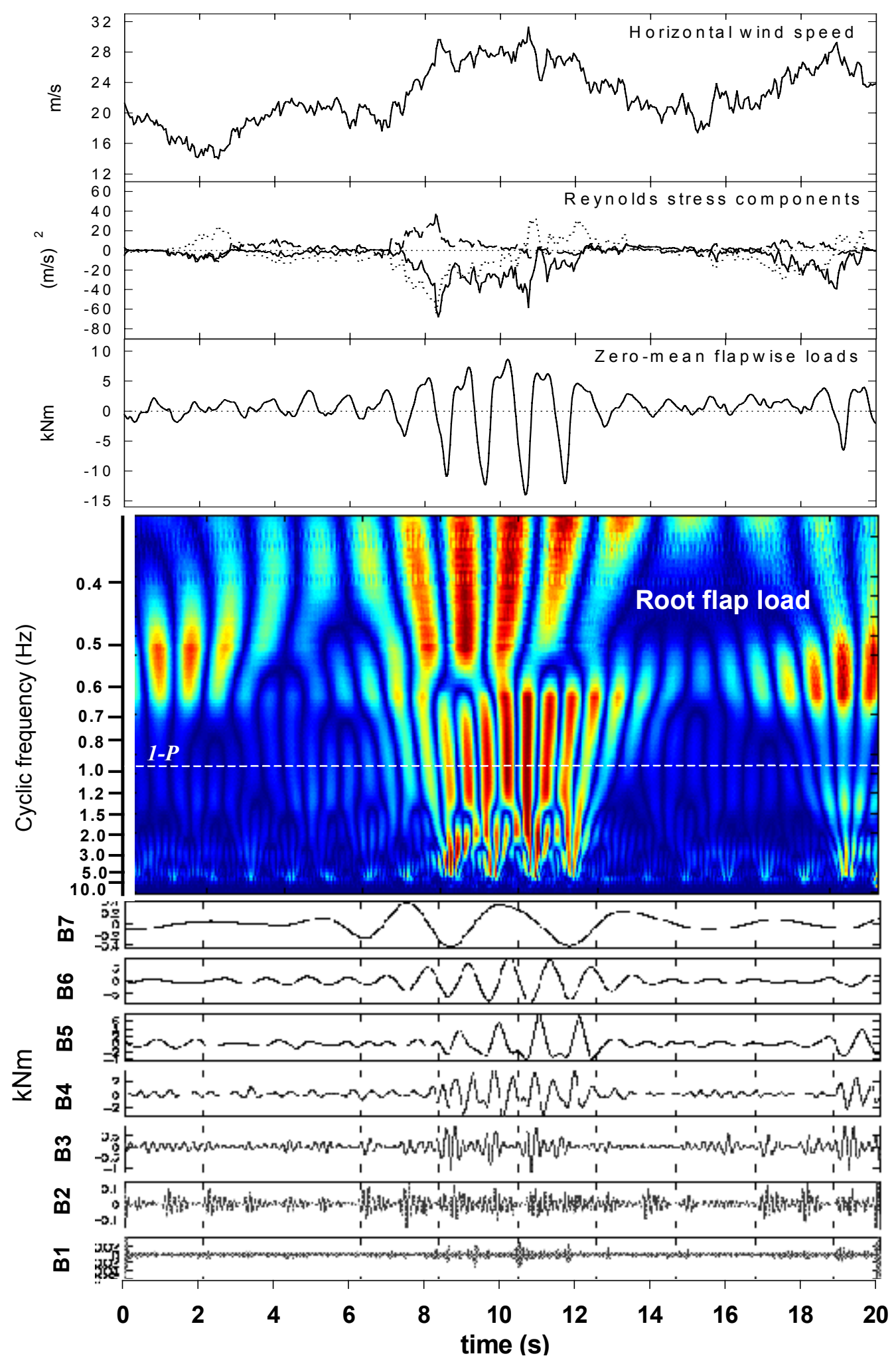

Figure 1-5. Time and wavelet analysis of simulated CWE300 turbine turbulence/rotor interaction 

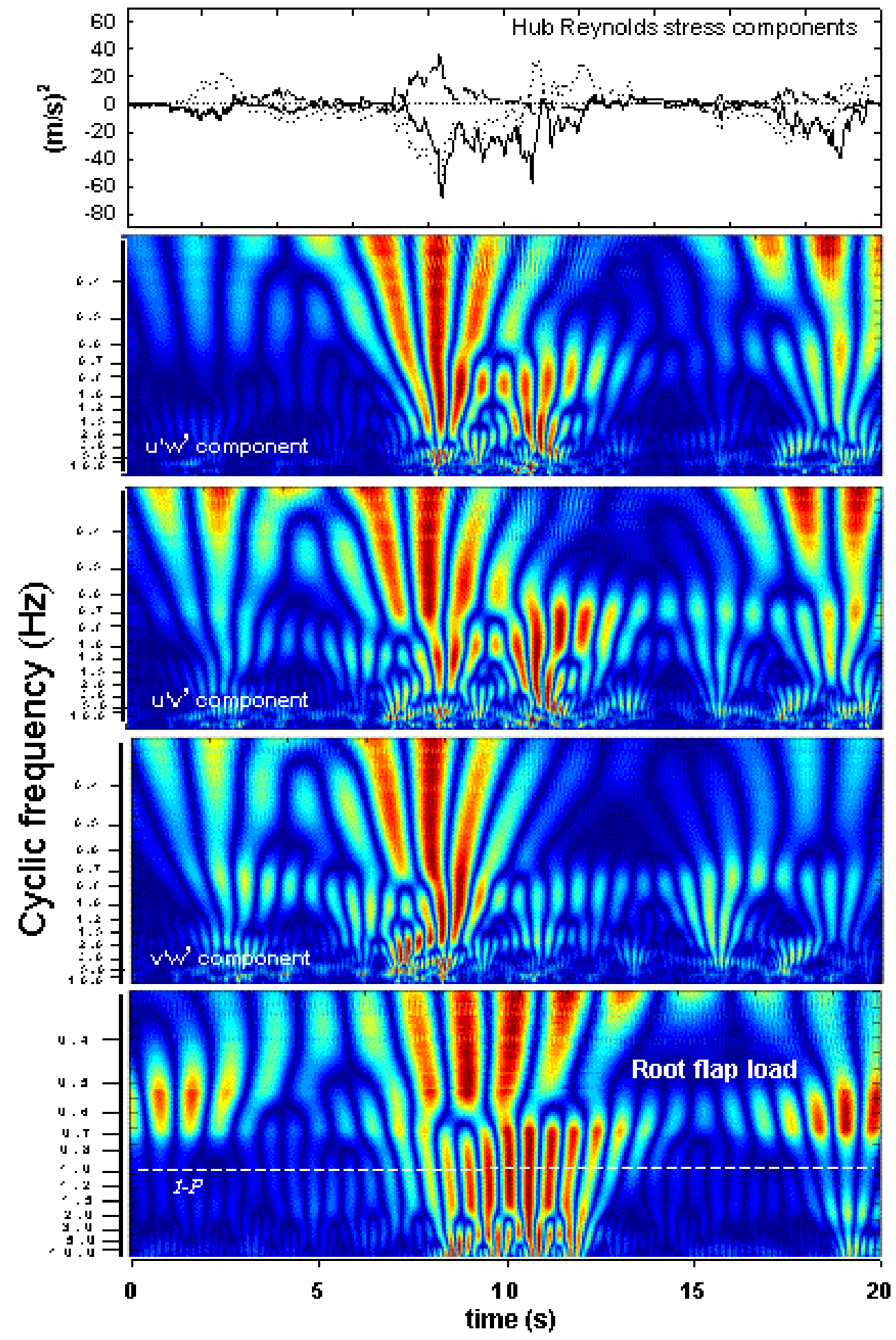

Figure 1-6. Continuous wavelet transform analysis of simulated CWE300 turbine turbulence/rotor interaction 


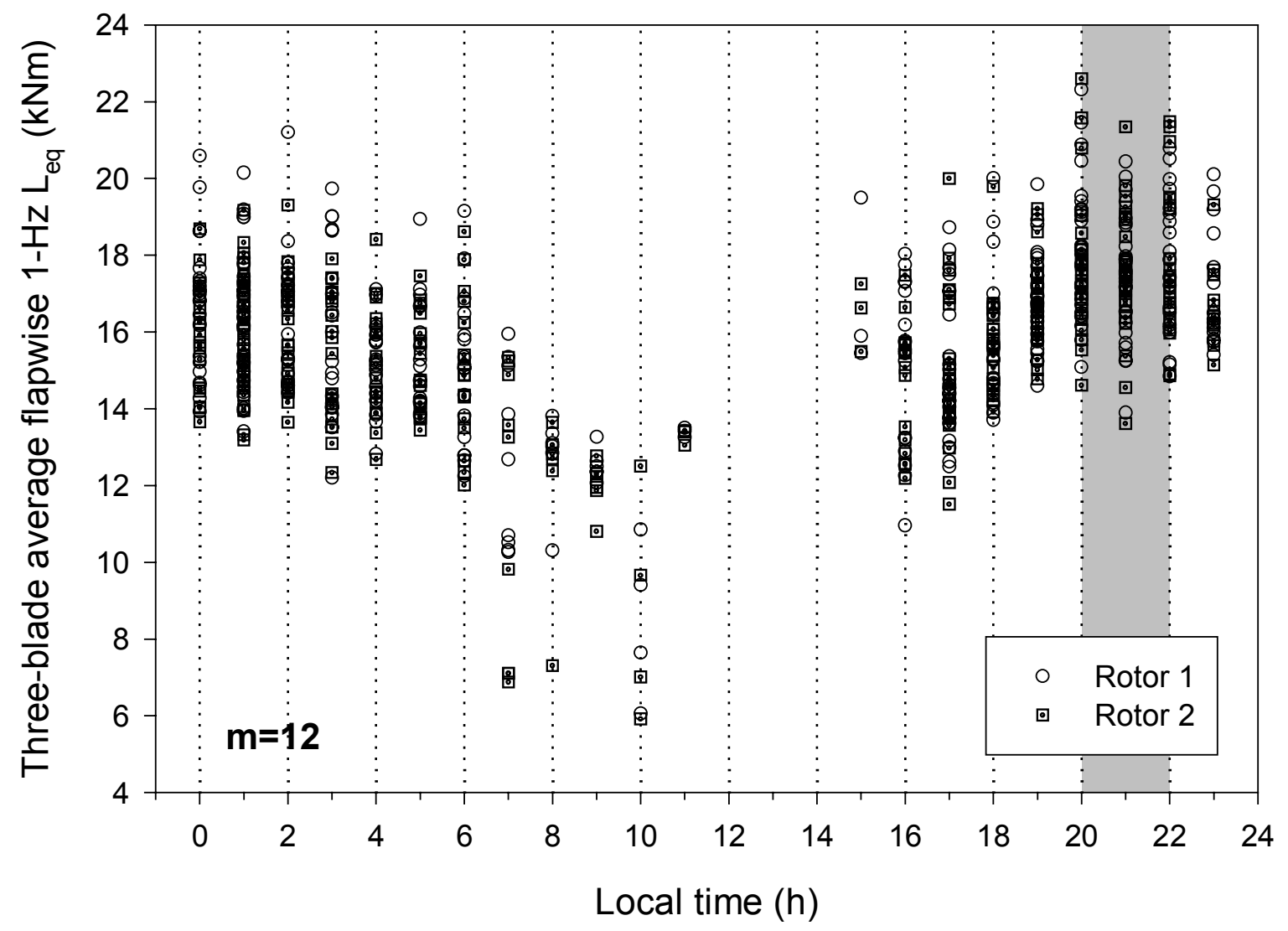

Figure 1-7. Diurnal variation of three-blade average flapwise root equivalent loads $L_{e q}$ for Micon 65/13 turbines in San Gorgonio Pass wind farm 


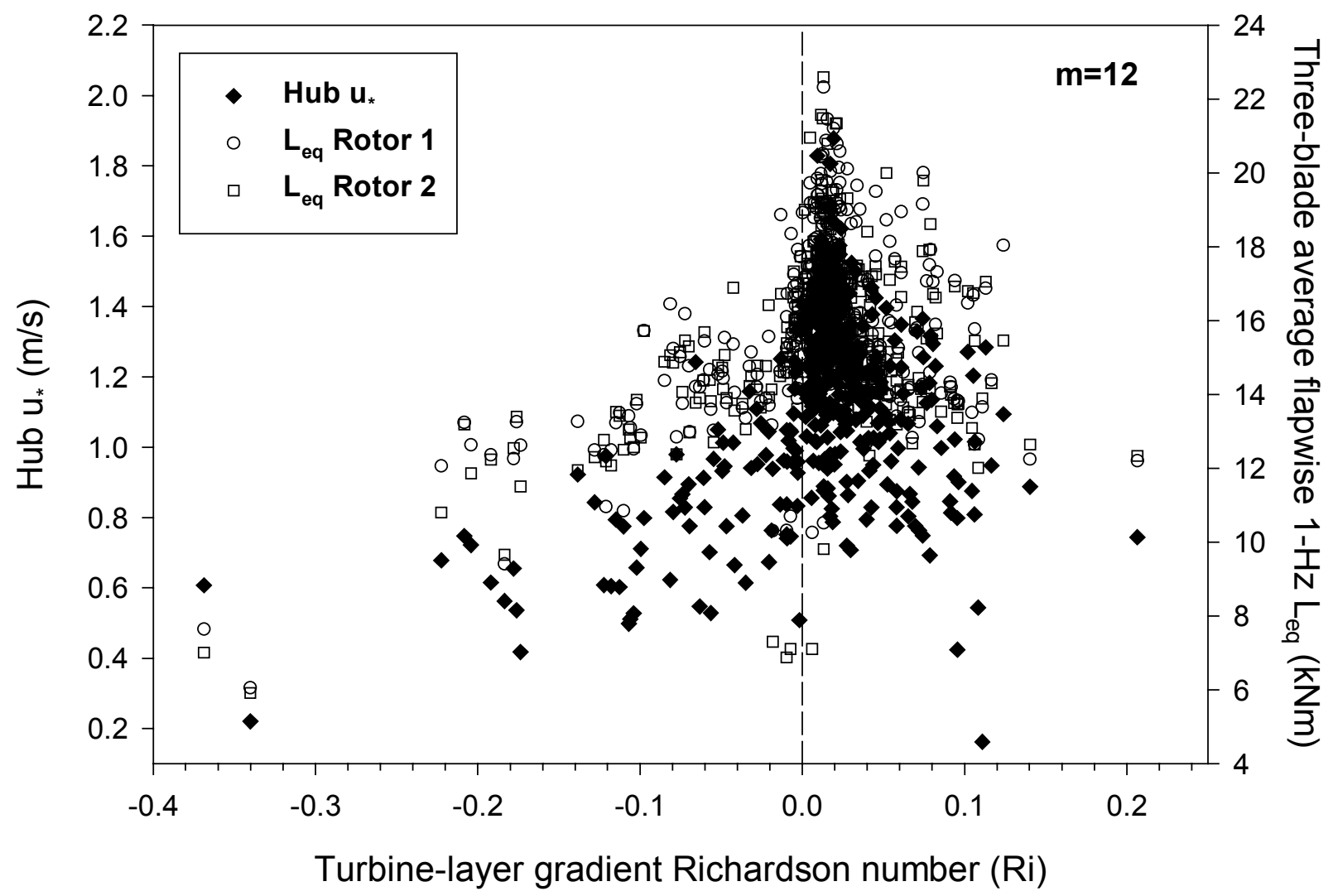

Figure 1-8. Variation of hub-height friction velocity $u_{*}$ and three-blade average flapwise equivalent loads $L_{e q}$ versus turbine layer gradient Richardson number (Ri) for Micon 65/13 turbine in San Gorgonio Pass wind farm 


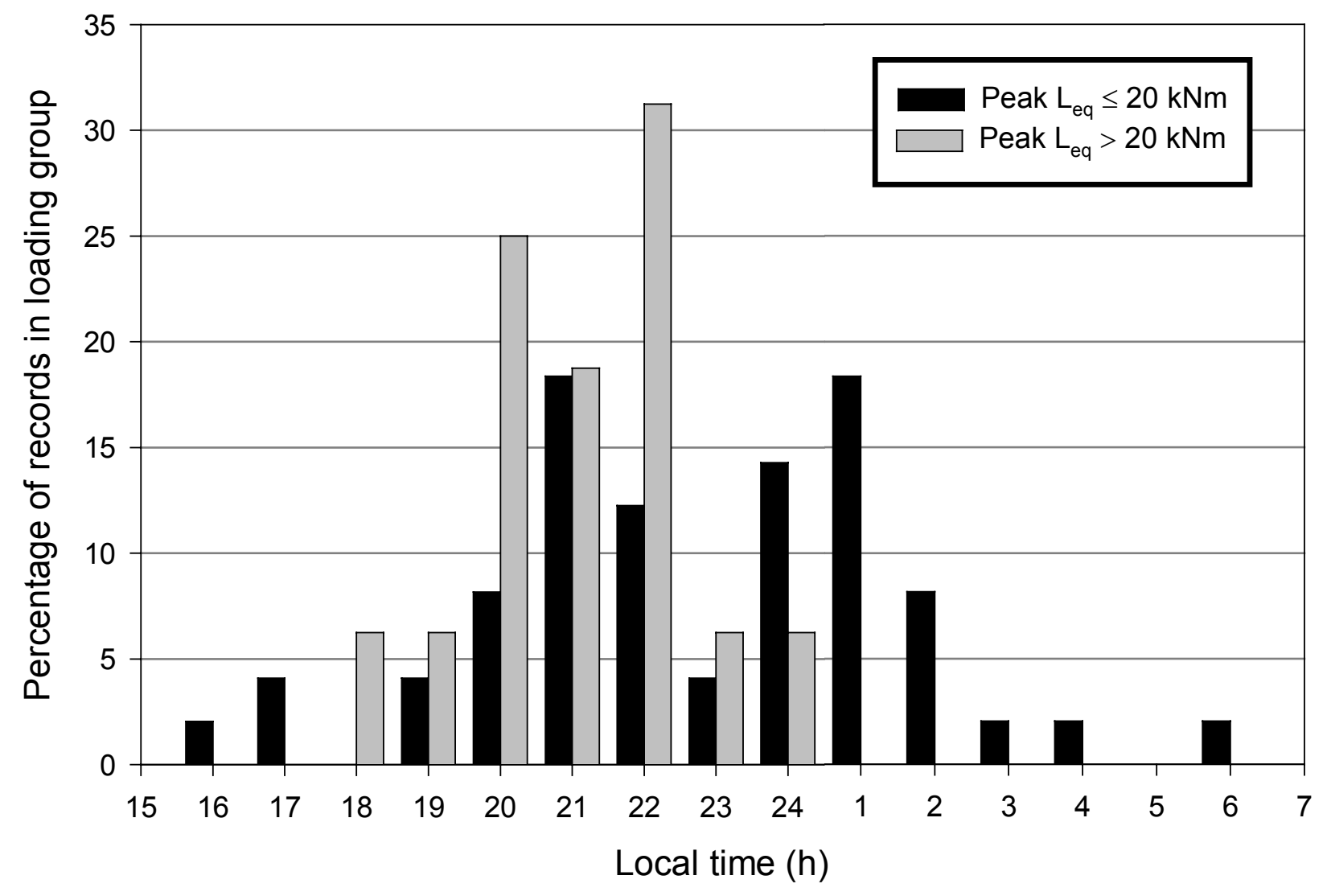

Figure 1-9. Time distribution of Micon 65/13 turbines peak loads by indicated groups 


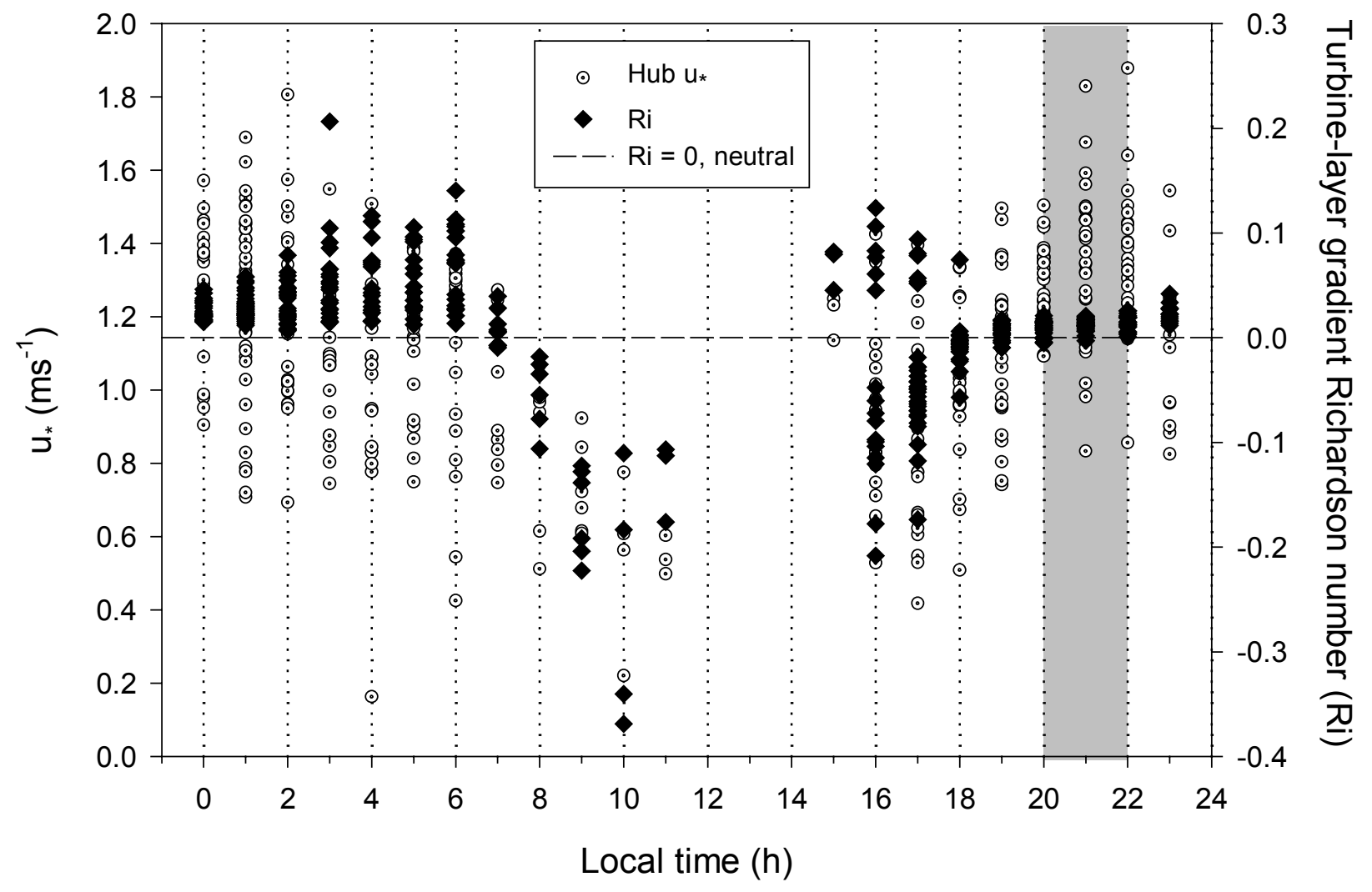

Figure 1-10. Diurnal variation of inflow Richardson number $(R i)$ and hub-height friction velocity $u_{*}$ for Micon 65/13 turbines in San Gorgonio Pass wind farm 


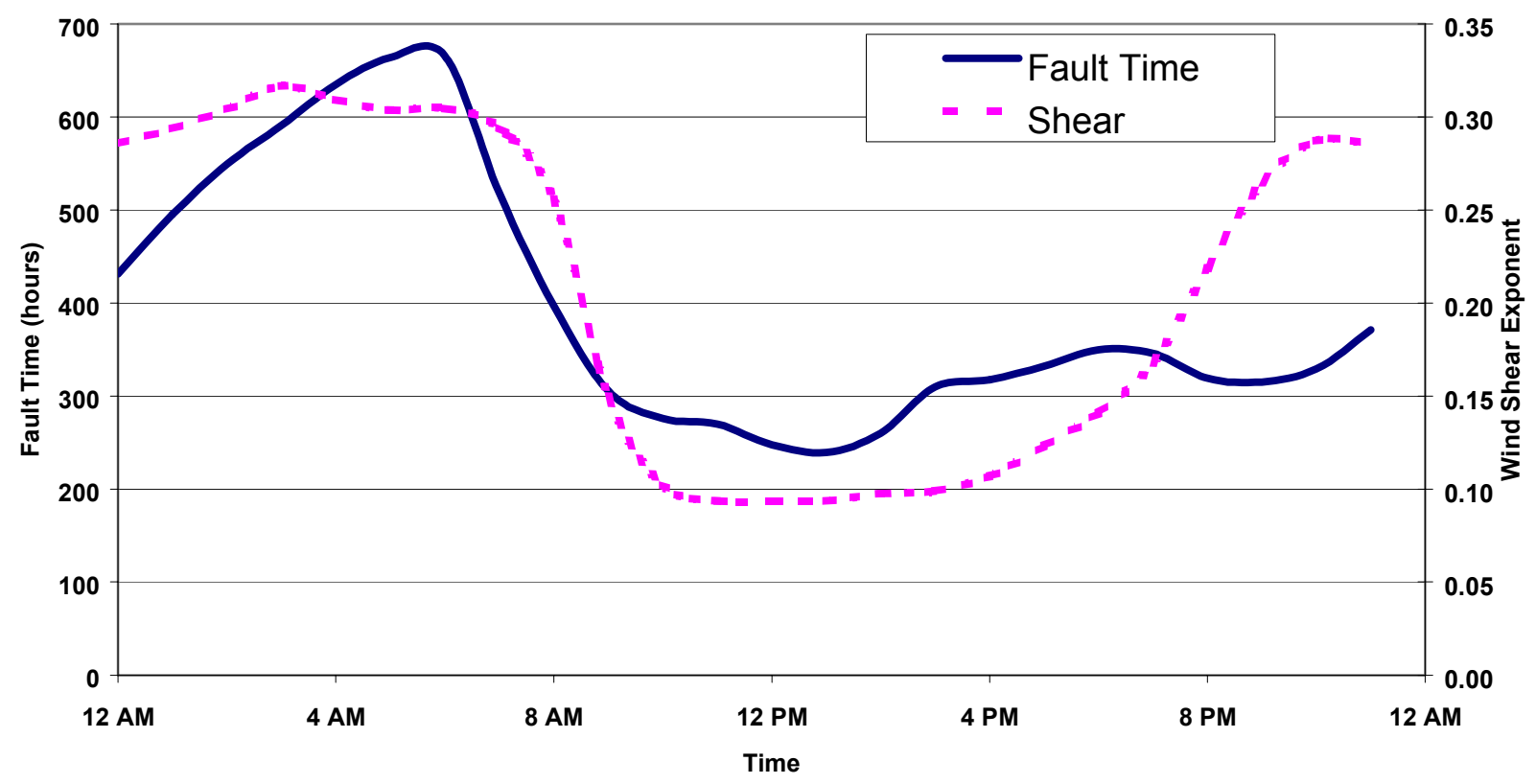

Figure 1-11. Diurnal fault and shear patterns at Big Spring, Texas (Source: $K$. Smith et al. [11]) 


\section{An Overview of the Atmospheric Boundary Layer}

Wind turbines operate in the lower portion of the atmospheric boundary layer (ABL). This is the layer of the atmosphere that is directly influenced by the land surface. During the day, the height of the boundary layer can extend up to $1-2 \mathrm{~km}$, but at night it becomes much thinner. Before dawn, it may only be $300 \mathrm{~m}$ deep and sometimes even less depending on surface conditions. There are two sub-layers that are of importance to wind-turbine operations. The surface layer occupies a relatively thin region near the ground, extending up to perhaps $100 \mathrm{~m}$ during the day, and is much lower at night. It is characterized by strong vertical gradients in wind, temperature, and moisture. The characteristics of the surface layer gradually modify with height and eventually become the mixed (or mixing) layer that extends almost all the way to top of the ABL. Here, the mean vertical gradients are much weaker. Thus wind turbines, particularly large ones, can operate in both regimes, sometimes simultaneously.

The surface layer is sometimes referred as the "constant flux layer" because the vertical transport or flux of momentum, heat, and moisture is more or less constant with height. These properties lead to what is known as Monin-Obukhov (M-O) similarity (or surface layer) scaling in which the turbulence properties scale with only a few variables. These variables include velocity, temperature, and length scales, given respectively by

$$
\begin{gathered}
u_{*}=\sqrt{\tau_{0} / \rho}, \\
T_{*}=-Q_{0_{v}} / u_{*} \\
L=-\frac{u_{*}^{3} \theta_{v}}{k g Q_{0_{v}}} \\
z
\end{gathered}
$$

where $\tau_{0}$ is the surface stress; $\rho$ is the air density; $Q_{0}$ is the surface heat flux; $k$ is a constant, usually given as $0.4 ; g$ is the gravitational acceleration; $\theta_{v}$ is the potential or thermodynamic temperature, which includes the effects of moisture; $L$ is the Monin-Obukhov length scale; and $z$ is height above the ground. Vertical motions in the surface layer generally obey similarity scaling, but horizontal motions do not, and the appropriate scaling relationships must be arrived at empirically. In similarity scaling, surface flow properties are non-dimensionalized by $u_{*}, T_{*}$ and $z$ and then become universal functions of $z / L$. The parameter $z / L$ is a measure of stability and, because of M-O similarity, is related to $R i$. The functional relationships between $u_{*}, T_{*}$ and $z$ have been determined from extensive field measurements. The SNLWIND-3D code employs surface-layer scaling to arrive at the target spectral models that are transformed into the time domain to produce the velocity components $u(y, x, t), v(y, z, t)$, and $w(y, z, t)$ used as input to turbine design code models [4]. Because temperature fields are not needed, only the $u_{*}, z$, and $z / L$ scales are used. For the neutral stability conditions associated with the Normal Turbulence Model (NTM) specified by the International Electrotechnical Commission (IEC) Standard 61400-1 [13] only parameters related to $u_{*}$ and $z$ are used because $z / L=0$. 
There is no consensus in the development of scaling relationships for mixed layer turbulence. Some workers have suggested a form of local scaling, using parameters similar to those used in the surface layer, but varying as a function of height (but not necessarily referenced to the ground) when moderate winds and slightly stable conditions exist. It is an objective of this program to utilize the long-term turbulence records collected to assess the desirability of this approach and make the necessary modifications in the SNLWIND-3D code to accommodate it.

\subsection{Daytime or Convective Boundary Layer}

The daytime boundary layer is characterized by rising air being heated from the surface below. Buoyant plumes of air establish convective, turbulent motions that can extend to the top of the ABL, where a capping inversion (stable layer) damps the motions. The scales of turbulent motions associated with the convective boundary layer are large relative to wind turbine dimensions (i.e., on the order of $1-2 \mathrm{~km}$ ). The mixing associated with the convective eddies largely smoothes out vertical gradients of wind and temperature. Shear exponents in a wellmixed convective $A B L$ are generally less than $1 / 7$. Surface-layer turbulence scaling is usually valid up to heights of $100 \mathrm{~m}$, and sometimes more.

\subsection{Neutral Boundary Layer}

If the vertical transport of surface heat and moisture throughout the ABL is negligible, the thermodynamic or potential temperature is invariant with height. Under such conditions, turbulence generation takes place only through the action of wind shear because there are no buoyancy effects. The real ABL is likely never truly neutral (except perhaps locally within some distinct layers) because some small amount of heat and moisture vertical transport always exists.

\subsection{Nocturnal or Stable Boundary Layer}

At a point in the late afternoon, the surface heat flux changes sign from positive to negative (i.e., heat moving away from the Earth to heat moving toward the Earth as the surface cools from radiative heat loss). Gradually, the air closer to ground becomes cooler than the air above. When this occurs, the resulting negative buoyancy damps the largest turbulent eddies, leaving the smaller ones largely unaffected. Without the large vertical motions associated with convection, the stable boundary layer stratifies into layers within which large vertical gradients of wind and temperature can exist. As compared with the convective boundary layer, the characteristic scale of turbulent eddies in the stable boundary layer is much smaller and becomes closer to the dimensions of wind turbines. The dynamic stability of the layers can vary depending on the gradients of temperature and wind speed involved. In cases of strong wind shear and weak temperature gradient, atmospheric wave motions can form, which in turn can generate organized turbulence as they become unstable (locally unbounded growth) and breakdown.

\subsection{Nocturnal Low-Level Jet Streams}

A common feature of the nocturnal boundary layer and one of great importance to wind energy operations is the low-level jet stream (LLJ) that forms in the stable mixed layer.

An example of the evolution of a LLJ as measured by an acoustic wind profiler is shown in Figure 2-1. The times of the indicated profiles are in local standard time. Clearly one of the main features of the LLJ is the significant shear below the wind speed maximum. Strong shears not only can have operational impacts on wind turbines as discussed by Eggers et al. [12], but are 
an important ingredient in the development of atmospheric wave motions and other non-linear flow phenomena that are sources of coherent turbulence in the nocturnal ABL.

\subsubsection{Low-Level Jet Climatology}

The relationship between the frequency of the LLJ and the available wind resource potential for the eastern two-thirds of the United States in plotted in Figure 2.2. The isopleths on this chart represent the number of jets observed over a 2-year period from a 1968 study by Bonner [14]. The blank area on the left of the figure reflects Bonner's not using weather stations in and near the Rocky Mountains because of their high elevations and expected small-scale wind systems. He used twice-daily rawinsonde (balloon-borne measurements of temperature, humidity, pressure, and the wind vector) observations ( 6 and $18 \mathrm{~h}$ Central Standard Time) to identify the presence and strength of low-level jets. More recent studies conducted by Mitchell et al. [15] and Whiteman et al. [16] used newer wind profiler technology and higher frequency rawinsonde observations (up to eight per day). While the general character of the spatial distribution of observed jets agreed well with Bonner's results shown in Figure 2-2, Whiteman found the jets tended to be stronger with the wind speed maximum nearer to the ground. Mitchell defined a warm season for jets from April to September. Whiteman found both southerly and northerly jets occur the year around. The southerly jets are more frequent with the maximum number occurring in the summer and at night. They display a distinct diurnal clockwise turning in wind direction and an oscillation in speed. Northerly jets are generally associated with cold air outbreaks behind southward-moving cold fronts, and their occurrence is more evenly distributed diurnally and do not exhibit a clockwise turning with time. Recently, Banta et al. [17] studied the low-level jet at a site in southern Kansas during October 1999. They found a high frequency of jets forming below $300 \mathrm{~m}$ with ones having higher wind speed maximums generally occurring at higher elevations.

Figure 2-2 shows a close correlation between the frequency of low-level jets and the wind resource potential in the Great Plains. It is, therefore, important to understand the linkage between the two and what operational impacts, if any, beyond the high vertical shears that may be encountered.

\subsubsection{Theory of Low-Level Jet Formation}

As the Earth's surface cools, the frictional drag on the wind imposed by the rising convective air currents disappears, allowing the wind speed to increase. Blackadar [18] proposed a mechanism that relates the acceleration of the wind to the degree of imbalance of forces resulting from the loss of the frictional drag when the surface heat flux reverses during the day-night transition. This acceleration causes the higher winds above the ground to separate or decouple from the air nearer the surface while increasing the wind shear. The accelerating air is turned to the right by the rotation of the earth (Coriolis force). This initiates an oscillation in the wind speed with a period of $\pi / \Omega \sin \phi$, where $\Omega$ is the angular rotation rate of the earth and $\phi$ is the latitude. The period of this oscillation (called an inertial oscillation) at the latitude of the LLLJP Site in southeastern Colorado is $18.4 \mathrm{~h}$. Thus, if the process started with the change in sign of the vertical heat flux with the accompanying sudden reduction in friction, the wind speed would reach a peak at a time equivalent to one-half of the inertial period or, in this case, about $9 \mathrm{~h}$ later. This is the time for the inertial oscillation velocity to align with the mean wind, and the observed speed is then the sum of the two. Further south this peak would occur later, and further north it would occur earlier. If the heat flux reversal was at $18 \mathrm{~h}$ local time and the oscillation actually 
began then, the peak in the wind speed would be observed near $03 \mathrm{~h}$ the next morning. Lundquist [19] recently completed a study of inertial oscillations, as well as their relationship to low-level jets. She found that velocities associated with inertial oscillations in the highly stable layer below $600 \mathrm{~m}$ contributed a large percentage of the observed jet wind speeds. She was also able to confirm the theory that nocturnal low-level jets tend to have inertial oscillations at the height of their peak wind speed. The example, LLJ shown in Figure 2-1 exhibits many of the characteristics discussed here (i.e., reaching the peak velocity between 03 and $04 \mathrm{~h}$ local time [about 8-9 hours after local sunset] and the clockwise turning of the wind as the jet evolves).

\subsection{Atmospheric Wave Motions}

The stably stratified nocturnal ABL is capable of supporting and propagating atmospheric wave motions. This is particularly true in the high-shear regions below LLJ maximums, as pictured in Figure 2-1a. In a stably stratified atmosphere, a parcel of air that is displaced upward will encounter a negatively buoyant restoring force that acts to return it to its original level. However, if the parcel accelerates downward and below its original height it will then be warmer 7and more buoyant than its surroundings. A positive buoyant force then attempts to restore it back up to its original level. Should this process continue, an oscillatory behavior results. This type of motion, under the right environmental conditions, creates atmospheric waves called gravity or buoyancy waves. Gravity waves can transport energy.

It can be shown [20] that the equation of motion for an air parcel displaced a small vertical distance $\delta z$ from its original height in a stably stratified boundary layer is given by

$$
\frac{d^{2}(\delta z)}{d t^{2}}=-\frac{g}{\theta} \frac{\partial \theta}{\partial z} \delta z
$$

where $\theta$ is the potential or thermodynamic temperature given by

$$
\theta=T_{a}\left(\frac{1000}{p}\right)^{0.286}
$$

where $p$ is the atmospheric pressure and $T_{a}$ is the air temperature at the original height.

If an air parcel is displaced vertically and released, its oscillatory motion is described by

$$
\delta z(t)=A e^{i N t}+B e^{-i N t}
$$

where

$$
N=\sqrt{\frac{g}{\theta} \frac{\partial \theta}{\partial z}} .
$$

If $N$ is real, it is the frequency of oscillation of the air parcel that is called either the buoyancy or Brunt-Väsällä frequency. Its units are in radians/s. Wave motion is possible only when the atmosphere is stably stratified (i.e., $\partial \theta / \partial z>0$ ). $N$ represents the maximum frequency for vertically propagating gravity waves. If $N$ is imaginary or $\partial \theta / \partial z<0$ the growth of the displacement is unbounded and is usually referred to as convective instability. 
Various types of instabilities can develop in the stably sheared flow underneath the LLJ jet maximum as described by Blumen et al. [21]. Additional references related to atmospheric wave motions may be found in [22, 23, 24, and 25]. One of the most common is Kelvin-Helmholtz $(\mathrm{KH})$ instability. Some investigators believe this mechanism is a major, if not the dominant, contributor to turbulence generation in the nocturnal boundary layer. Linear stability theories of stably stratified parallel shear flows demonstrate that a necessary condition for the development of $\mathrm{KH}$ instabilities is $R i<0.25$. The gradient Richardson number equation may be alternately written as

$$
R i=\frac{g}{\theta}\left(\frac{\partial \theta}{\partial z}\right)\left(\frac{\partial U}{\partial z}\right)^{-2}=N^{2}\left(\frac{\partial U}{\partial z}\right)^{-2} .
$$

Equation (2-9) emphasizes the relationships between shear and buoyancy oscillations. KH instabilities form between two much-more stable layers that act as lids, which do not let the wave motions propagate away from the layer in which they are generated. This type of wave motion is called non-propagating or trapped. Because of these limiting boundaries, the wave cannot grow vertically outside the boundaries and rolls over instead. As a result, they are called K-H billows. At some point the billow circulation becomes unstable as some of the original, warmer and less dense air at the top of the boundary is transported downward while colder, denser air replaces it, thus creating a local convective instability (i.e., the local $R i$ becomes negative). Turbulence is then generated in these convective regions even though the background is stable. Eventually the whole billow breaks down into small, intense coherent circulations that act to smear out or at least reduce intensity of the original vertical shear.

Figures 2-3a, b, c present a numerical simulation of the evolution of the non-dimensional temperature field associated with the lifecycle of a stationary $\mathrm{KH}$ billow. This simulation was created by the National Center for Atmospheric Research (NCAR) using their Large-EddySimulation (LES) Code [26] and is based on the work of Werne and Fritts [27]. The areas of equal temperature approximate streamlines in this presentation. The simulation used a computational domain whose dimensions were $(180,60,180)$ nodes in the $(x, y, z)$ dimensions representing non-dimensional dimensions of $(4 \pi, 4 / 3 \pi, 25)$. Periodic boundary conditions are imposed on the horizontal directions $x$ and $y$. The domain was initially stress free with fixed temperatures imposed at the top and bottom. The streamwise background flow is defined as

$$
u=U_{0} \tanh (z / h)
$$

where $U_{0}$ and $h$ are constant velocity and length scales and $z$ is the vertical direction. The background temperature is linear $T=\beta z$, where $\beta$ is the mean vertical gradient. A total of 6000 time steps were used to describe the process.

The graphs of Figures 2-3a, b, c present the non-dimensional temperature field in the $x$ - $z$ plane at $y=30$ and time step increments of 200 up to time step 5801. The contours of non-dimensional temperature are equivalent to streamlines and reveal the basic flow structure as it evolves. At the first time step, the flow is parallel with the coldest temperature (dark blue) at the bottom of the layer the warmest (dark red) at the top. A disturbance has triggered the billow development at time step 201. By time step 401 the circulation has begun to bring colder air above warmer increasing the instability. The flow begins to show the development of secondary instabilities at the boundary of warmer and colder air at time step 801, which intensify at time step 1001. Much larger turbulent structures are evident at time step 1201 that spread out by step 1401. The billow 
begins to breakdown at time step 1801 and becomes fully turbulent by time step 2401 in Figure 2-3b. Between time steps 2601 and 3801 the flow remains very turbulent with distinct structures evident particularly near the upper and lower boundaries. These structures reach a peak between time steps 4001 and 4401, when a re-stratification begins. At time step 4601 through 5801, a more distinct stratification forms with secondary instabilities now forming near the top and bottom of the main billow remnants. Thus, the turbulent structures associated with lifecycle of a $\mathrm{KH}$ billow range in size from large-scale vortex circulations of the billow depth to much smaller, intense coherent structures resulting from secondary instabilities.

Highly stratified shear flows do not always lead to active KH billow formation. Flows with high shears may remain stable if the value of the layer $R i$ remains too large. Situations in which a very intense shear is coupled with small values of $R i$ (weakly stable) are likely to produce strong, rapidly growing $\mathrm{KH}$ wave activity and subsequent coherent turbulence generation. Thus wind turbine rotors encountering active KH billows are exposed to a wide range of coherent turbulent structures that likely will excite the natural frequencies (sometimes simultaneously) of the turbine structure resulting in a narrowband response. 

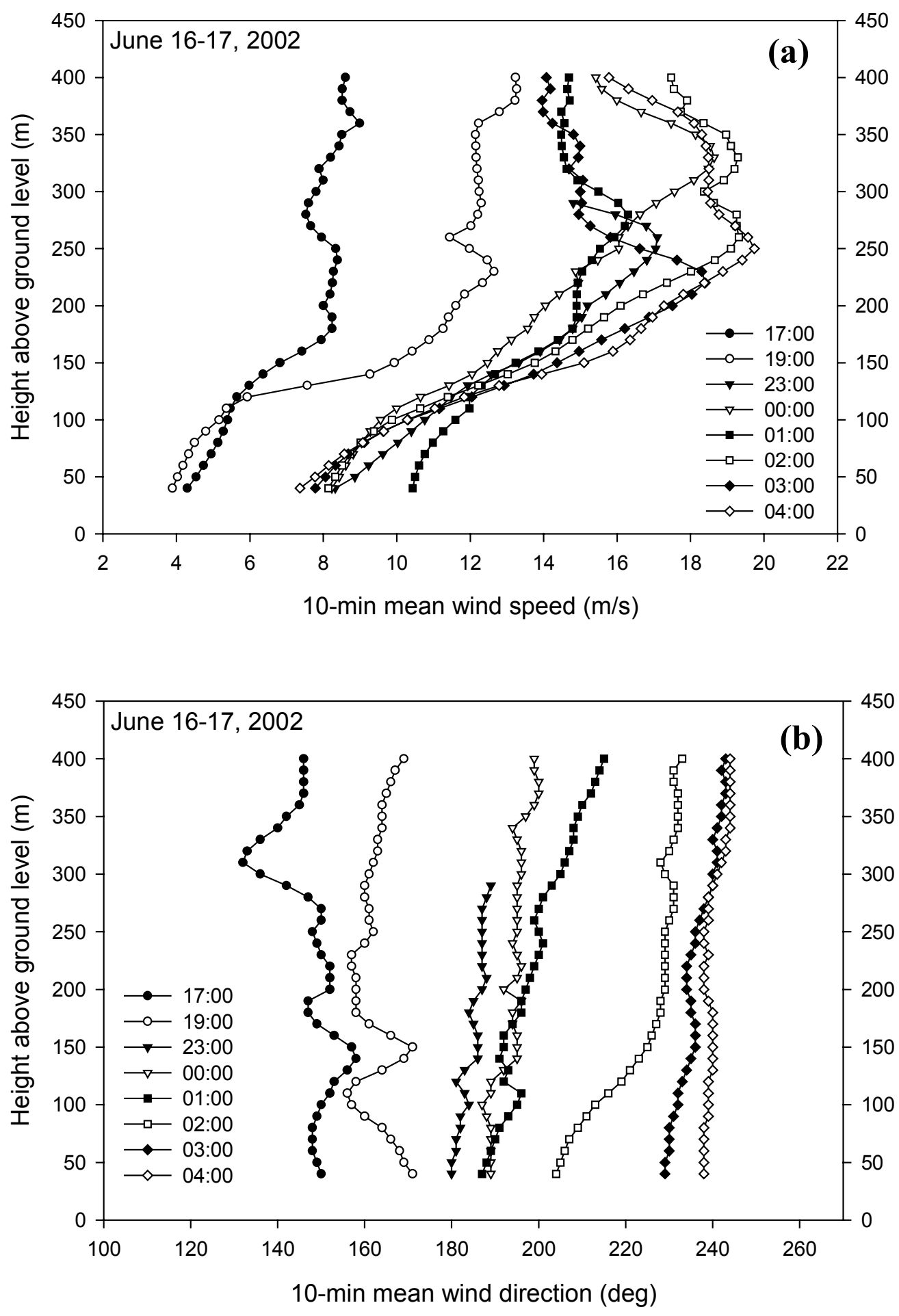

Figure 2-1. Example of low-level jet evolution from SODAR-derived 10-minute mean (a) wind speed and (b) direction for LLLJP Site on 16-17 June 2002 


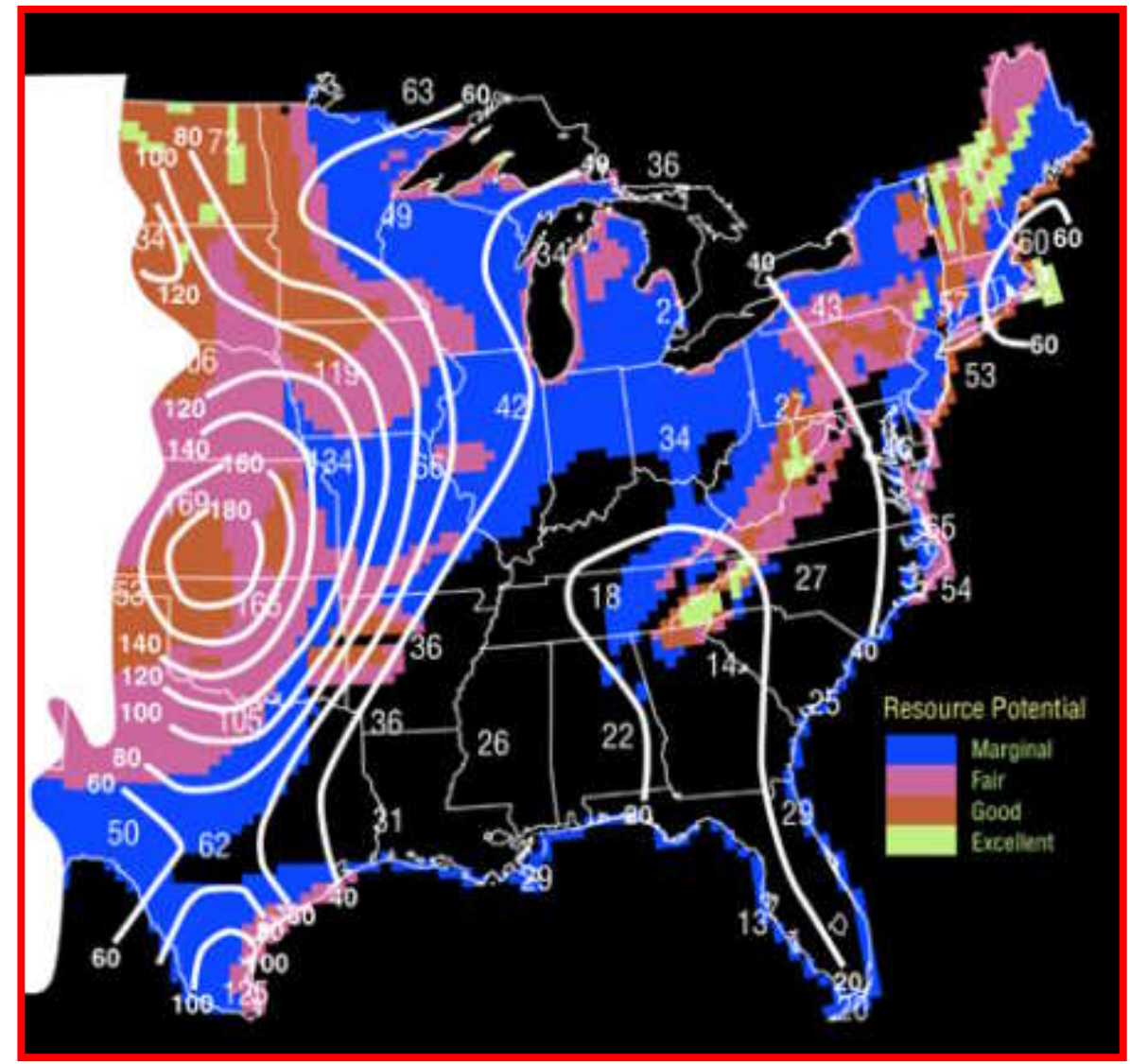

Figure 2-2. Overlay of biannual low-level jet frequency and wind resource map for eastern two-thirds of the United States (Low-level jet data source: Bonner [14]) 

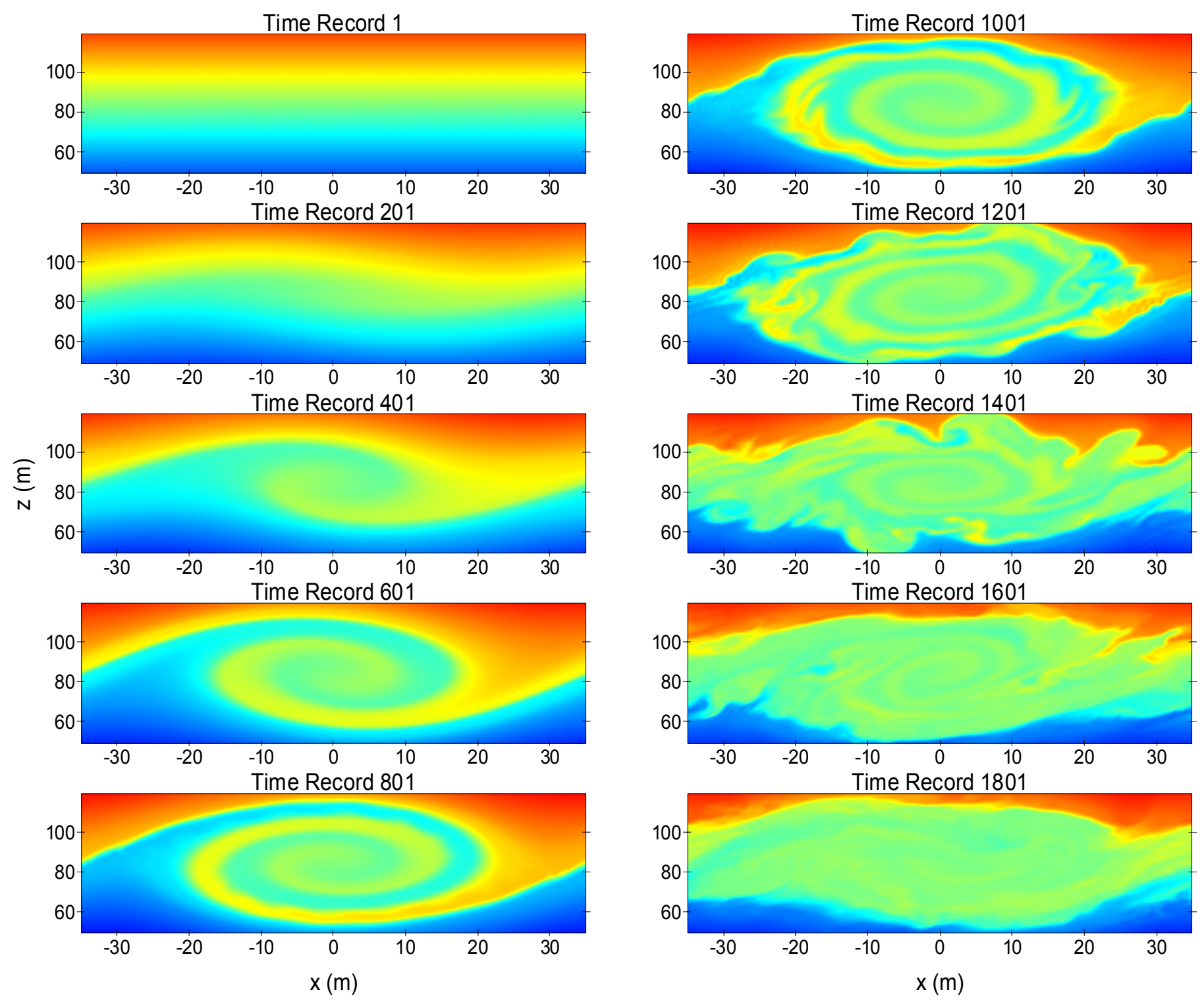

Figure 2-3a. Non-dimensional temperature field of KH billow life cycle 

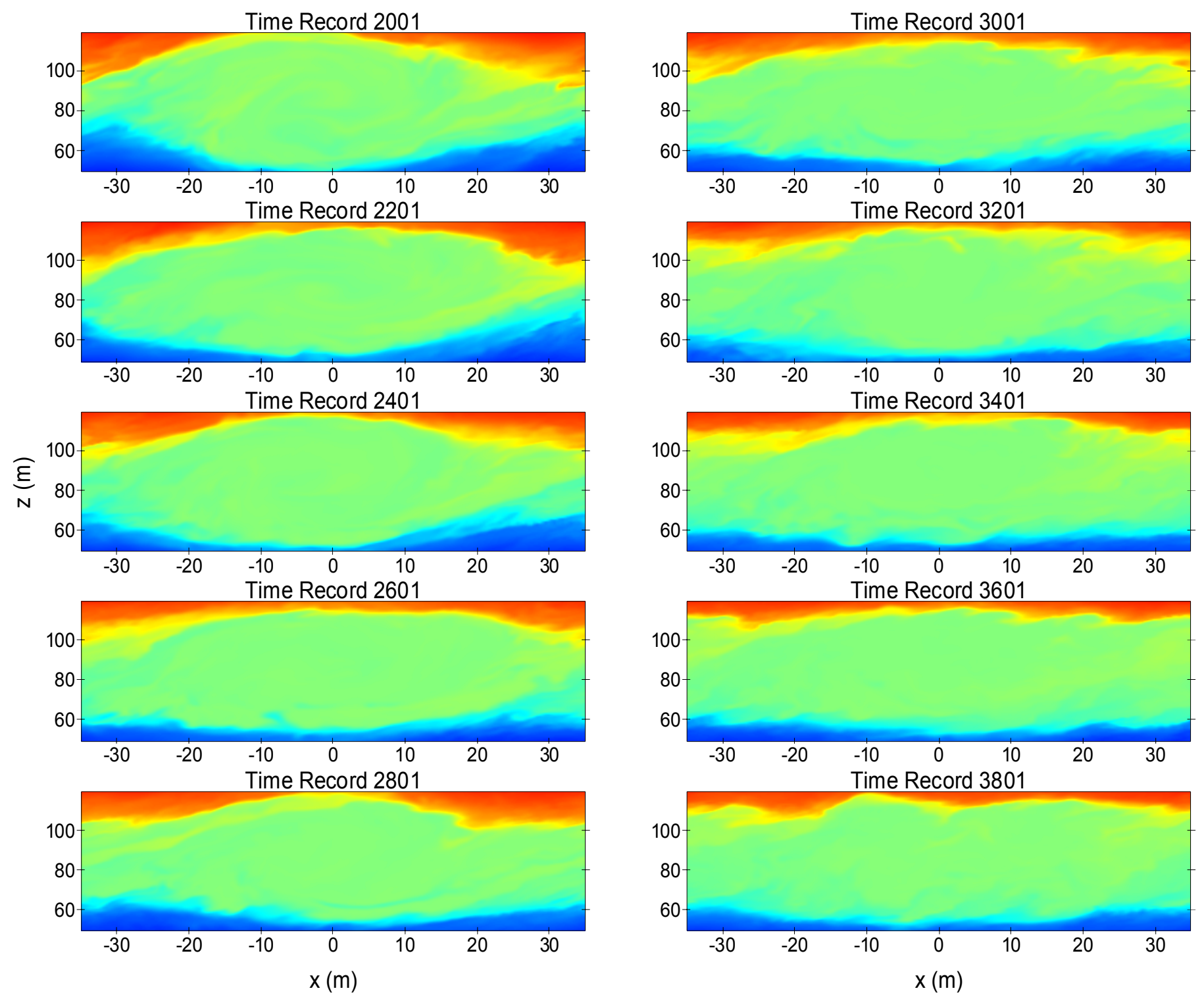

Figure 2-3b. Non-dimensional temperature field of KH billow life cycle 

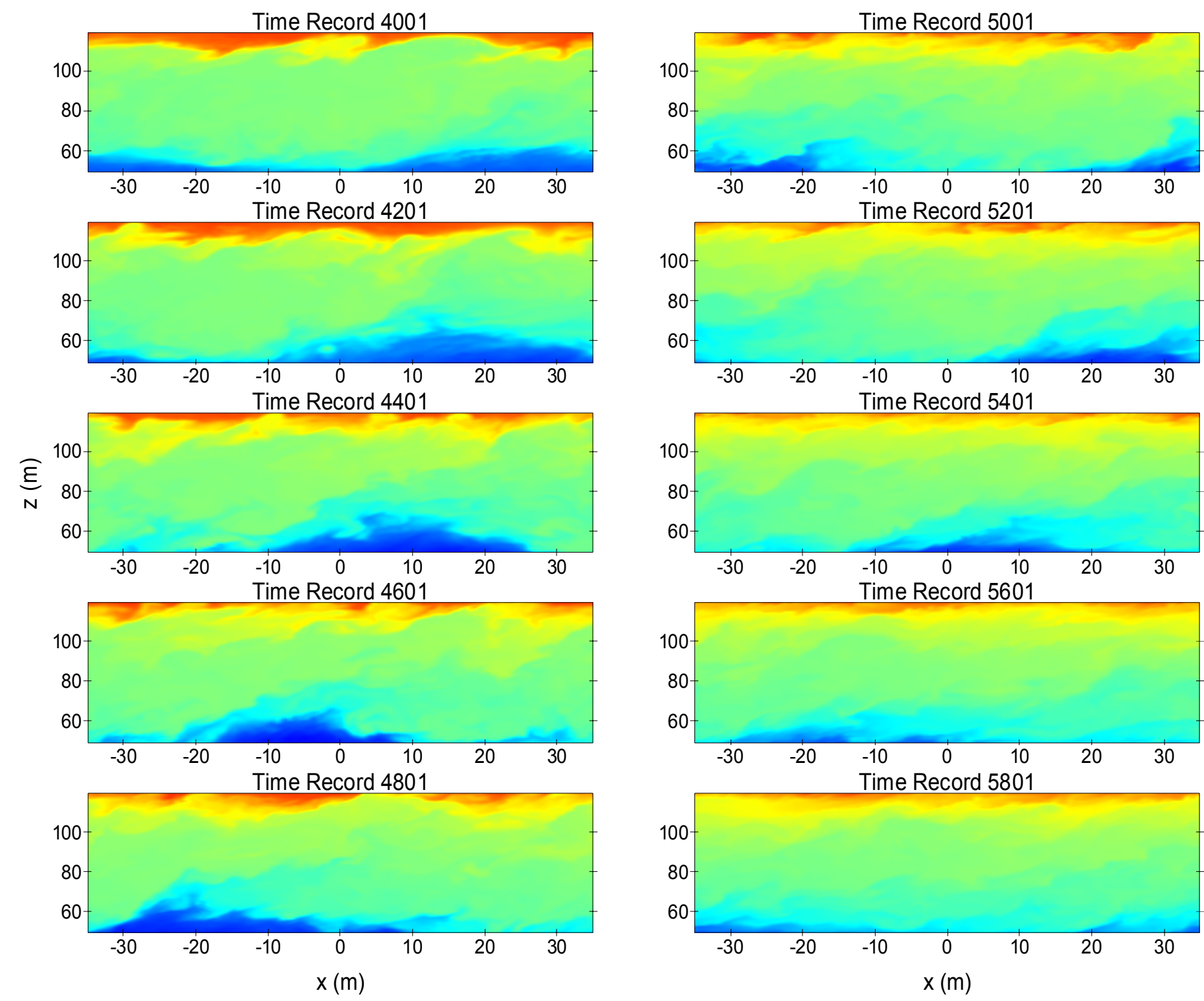

Figure 2-3c. Non-dimensional temperature field of KH billow life cycle 


\section{Analysis of NWTC/LIST Program Results}

In order to better interpret the conditions in terms of potential wind operational impacts seen at the Lamar Low-Level Jet Project (LLLJP) Site in southeastern Colorado, we performed a limited analysis on the available data from the NWTC/LIST Program. A total of 1570 10-minute records exist in which the ART turbine operated continuously and the hub-height mean wind direction was within $\pm 45^{\circ}$ of the perpendicular to the upstream sonic anemometer measurement array. Our objective is to use the ART results to estimate what it may encounter if it was operating in the LLLJP environment.

The wind conditions at the NWTC have been frequently described as "rigorous." While the annual mean wind speed is low $(\sim 3-4 \mathrm{~m} / \mathrm{s})$, the site is often buffeted by strong downslope flows emanating in the lee of the Front Range of the Rocky Mountains immediately to the west. The reader is referred to articles by Lilly and Zipser [28, 29] and Banta et al. [30] for discussions of the characteristics of the energetic flows experienced at the NWTC. Monti et al. [31] discuss the results of an observational study of the flow characteristics and accompanying turbulence over slope under nocturnal boundary conditions.

\subsection{A Simple Linear-Regression Model of the ART Turbulence-Induced Response}

In order to assess the potential impact of the LLLJP Site environment on the operation of 1.5MW turbines to be installed in the future, we employed a simple modeling process based on stepwise multiple linear regression to identify a minimum set of bulk statistical parameters that can serve as independent or excitation variables of the statistical response of the ART turbine. These variables then would then collectively explain a significant portion of the observed variance in the ART turbine response distribution. We will then use the resulting model with the same independent variables measured at the LLLJP Site to estimate what response the ART turbine would experience if it were installed in that environment.

\subsubsection{Identification of Inflow (Excitation) and Response Variables}

We chose five candidate inflow variables to serve as excitation variables and four as response variables related to the blade root flapwise bending, including the equivalent load $L_{e q}$, the maximum alternating load cycle $L_{p-p}$, the extreme load $L_{p k}$, and the extreme or peak zero-mean load $\hat{L}_{p k}$. The five inflow excitation variables chosen include:

- Hub-height horizontal mean wind speed, $\overline{U_{H}}$

- Hub-height vertical wind speed standard deviation, $\sigma_{w}$

- Turbine-layer gradient Richardson number, $R i$

- Hub-height peak coherent turbulence kinetic energy, $E_{c o h}$

- Turbine layer buoyancy scale, $l_{b}$.

The mean horizontal wind speed is defined as

$$
\overline{U_{H}}=\left(\overline{u^{2}+v^{2}}\right)^{1 / 2}
$$


where $u$ and $v$ are the streamwise (longitudinal) and cross-wind (lateral) wind components measured at hub-height. The turbine layer $R i$ is defined as the gradient Richardson number calculated over the layer from $3 \mathrm{~m}$ to just above the top of the turbine rotor $(61 \mathrm{~m})$. We did not include the hub-height turbulence intensity $\left(T I=\sigma_{H} / U_{H}\right)$ as a predictor candidate because previous experience [2] and other analyses found its relationship to root flapwise loads was of low significance and the correlation was actually negative (i.e., high values of $T I$ were correlated with low load excursions and vice versa). We believe this behavior is the result of $\sigma_{H}$ increasing at a slower rate than the corresponding increase in $U_{H}$ resulting in smaller values of $T I$ in higher wind speeds. We found [2] significant sensitivities to $\sigma_{H}$ in the Micon 65/13 turbines tower axial bending and nacelle inplane thrust loads, but the Reynolds stress components $U_{H}$, and $R i$ provided the best correlations with the observed load parameters.

Because we believe that coherent turbulent energy plays a major role in turbine response, we define the variable coherent turbulent kinetic energy as

$$
E_{c o h}=1 / 2\left[\left(u^{\prime} w^{\prime}\right)^{2}+\left(u^{\prime} v^{\prime}\right)^{2}+\left(v^{\prime} w^{\prime}\right)^{2}\right]^{1 / 2}
$$

which is similar to the more common definition of turbulent kinetic energy (TKE)

$$
E=1 / 2\left(u^{\prime 2}+v^{\prime 2}+w^{\prime 2}\right)
$$

where $u^{\prime}, v^{\prime}$, and $w^{\prime}$ respectively represent the streamwise, crosswind, and vertical turbulence or eddy wind components in a coordinate system aligned with the mean streamline and in the direction of the mean shear. As defined, $E_{c o h}$ represents the portion of the available TKE that has a coherent structure associated with it. If the turbulent field is homogenous isotropic (i.e., fully random with no correlations between the components), then $E_{c o h}=0$. Also $E_{c o h} \leq E$ with the instantaneous ratio of $E_{c o h} / E$ typically 0.5 or less in flows with coherent elements. $E_{c o h}$ provides a convenient variable to compare turbulence response with and, in this case, we use the maximum or peak value of $E_{c o h}$ seen in a 10 -minute record. Like $E, E_{c o h}$ has the units of energy per unit mass or $\mathrm{m}^{2} / \mathrm{s}^{2}$ associated with it.

We hypothesize that instabilities associated with stably sheared flows that are present in the atmosphere below nocturnal jet maxima are a significant source of coherent turbulent structures and may have important operational impacts on turbines. We also suspect that KH instability may frequently be present, and we need a measure related to it to include in our regression model. From our previous discussion in Section 2.5, we know the gradient Richardson number being in the critical range (i.e., $0<R i<0.25$ ) is a necessary condition for $\mathrm{KH}$ instability, but it does not provide any insight into the size or space scales the KH billows may take place. A parameter that indicates the spatial scale over which the degree of suppression of vertical motions by static stability is the buoyancy length scale or $l_{b}$ is given by

$$
l_{b}=\frac{\sigma_{w}}{N}
$$

We refer to $l_{b}$ as the either the turbine layer or rotor layer buoyancy scale depending on whether it is calculated from near the ground to the top of the rotor or only over the heights occupied by the rotor respectively. According to Stull [32], it can range from a couple of hundred meters in 
weak stratification (small $N$ or small $R i$ ) to less than a meter in strong stratification when the buoyant damping is substantial. Thus, a weakly stable stratification (small $R i$ ) and a large value of $\sigma_{w}$ indicate the likely presence of large coherent motions. When plotting, we normalize the value of $l_{b}$ by the ART rotor diameter $(D=43 \mathrm{~m})$. In this way we have a measure of the scale of the coherent disturbances relative to the turbine rotor dimensions.

Table 3-1 summarizes the mean values for the five inflow predictors and four response variables by assigning a stability class to each of the 10-minute 1540 records. While only four classes are shown ( 2 through 5), Class 1 would include records in which $-1 \leq R i$, none of which occurred in this particular data set. The table indicates a predominance of records occur in Stability Class 3, which can be considered weakly stable conditions.

\subsubsection{The Stepwise Regression Results}

We applied stepwise multiple linear regression to each of the response variables individually, initially including all five of the independent variables or predictors. The object was to obtain a relationship with the fewest number of predictors consistent with the highest statistical significance. Measures of statistical significance included the $F$-statistic and the coefficient of multiple determination (multivariate correlation coefficient $r^{2}$ ) for the model, and the $t$-value and significance level for each predictor. The $F$-value was calculated from the ratio of the mean square of the regression to the mean square of the residuals. The $t$-value is used to test the null hypothesis that the associated coefficients are equal to zero (i.e., no influence on or correlation with the response variable). The significance level for each coefficient is then used to gauge whether the estimated coefficient may have occurred by chance alone. The significance level of each coefficient indicates the probability of encountering a $t$-value as large or larger in magnitude than the one associated with the null hypothesis, which states that the coefficient is zero. A very small significance level is desirable and indicates the predictor is highly correlated with the response variable. This technique will fail if there is a high degree of correlation (colinearity) between the predictor variables. Ideally, it is desirable for the predictor variables to be independent of one another, but that is rarely possible with meteorological variables. We successively reapply the regression until we are left with those predictors that exhibit very low values of the significance level (typically 0.0001 , but less than 0.05 is required) coupled with maximums of the $F$-statistic, $t$-value, and $r^{2}$.

The linear correlation coefficient matrix $R_{i j}$ for the five inflow predictors is shown in Table 3-2. The linear correlation coefficient is defined as

$$
R_{i j}=\frac{\overline{x_{i} x_{y}}}{\sigma_{i} \sigma_{j}}
$$

where $x_{i}$ and $x_{j}$ are two variables being compared with one another respectively. $R_{i j}$ can vary over the range of \pm 1 , where a value of +1 indicates a perfect positive correlation between $x_{i}$ and $x_{j}$ and a value of -1 a negative one. It allows conclusions regarding the existence of a linear relationship between variables, but does not indicate which variable influences the variation of the other. In comparison, the coefficient of determination (multivariate correlation coefficient) is defined as 


$$
r^{2}=1-\frac{S S_{r e s}}{S S_{t o t}}
$$

where $S S_{\text {res }}$ is the sum of the squared deviations from the fitted regression line, and $S S_{\text {tot }}$ is the sum of squared deviations from $\bar{x}_{i}$ or a measure of its total variation. It describes how well a straight line fits the variation between $x_{i}$ and $x_{j}$ with 0 being none to +1 being perfect (i.e., a true linear relationship).

In Table 3-2, with the exception of $\sigma_{w}$ and $E_{c o h}$, matrix elements $R_{i j}$ are not highly correlated with one another. We believe the high correlation of $\sigma_{w}$ and $E_{c o h}$ results from the vertical velocity field being associated with atmospheric wave motions (which are often largely twodimensional [i.e., dominant dimensions in the horizontal and vertical]) in a stable atmospheric layer, as well as turbulent motions in the convective boundary layer. The linear correlation coefficients $R_{i j}$ between the five inflow predictors and the four response variables are shown in Table 3-3. Because they are all derived from the same basic root parameter (i.e., the flapwise bending load), there are only slight variations with each response variable for each of the inflow predictors. The exception is the lower correlation value between $U_{H}$ and the peak zero-mean load $\hat{L}_{p k}$. We interpret this to mean the effect of the mean angle of attack has been removed when the mean load is removed and what correlation remains is a result of turbulent fluctuations.

Table 3-4 summarizes the relative sensitivities of each of the response variables to the five inflow predictors. Clearly, $\sigma_{w}$ is an important parameter in characterizing the root flapwise response for this data set. This is not surprising if, as we believe, coherent motions associated with KH instability are dominating the large number of data records (listed in Table 3-1 as 1079 or $69 \%$ of the total) collected when the turbine layer dynamic stability was weakly stable (i.e., 0 $<R i \leq 0.10$ ). KH billow structures tend to be largely two-dimensional (at least early in their lifecycle) with most of the energy in the $u$ and $w$ wind components associated with the waveinduced shearing stress $\rho u$ ' $w^{\prime}$. Figure 3-1 plots the observed relationships between $\sigma_{w}, R i, l_{b} / D$, and peak $E_{c o h}$ for stable flows $(R i>0)$. The strong correlation between $\sigma_{w}$ and peak $E_{c o h}$ is obvious. Figure 3-2 plots the observed variation of flapwise $L_{e q}$ against $U_{H}, \sigma_{w}, R i$, and $l_{b} / D$ also for stable flows. Again, the strong correlation between flapwise $L_{e q}$ and $\sigma_{w}$ is well marked.

\subsection{Interpretation of NWTC/LIST Results}

The relationship between flapwise $L_{e q}, \sigma_{w}$, and peak $E_{c o h}$ for the full range of observed $R i$ is plotted in Figures 3-3a and $b$. Locally weighted regression was employed to identify the underlying variation of these variables with stability. Figure 3-4a plots the resulting smoothed relationships for the whole operating range of conditions, whereas Figure 3-4b presents the same information when the turbine was operating in below-rated conditions (i.e., there was no pitch control activity because the wind speed was below rated level). The unstable to stable transition is much more pronounced when the records with higher wind speeds are included. There is a dramatic increase in flap $L_{e q}$ accompanied by similarly rapid increases of $\sigma_{w}$ and peak $E_{c o h}$ when $R i$ decreases below +0.1 and reaches a peak below +0.05 for the inclusive case (Figure 3-4a). There is a dramatic drop as the $R i$ becomes negative (convective). For the below-rated records, the transition is much more gradual, but again it follows a similar pattern with the peak value of flap $L_{e q}$ occurring at $R i$ between 0 and +0.05 . Thus, increased fatigue can be expected when the 
turbine is operating in above-rated conditions in this range of weak stability as a result of the high values of $\sigma_{w}$ and peak $E_{c o h}$ (coherent turbulence) present. It is precisely this range of dynamic stability that produces the most energetic levels of $\mathrm{KH}$ instability and the coherent turbulence generation that accompanies it. The plot of the rotor-diameter normalized buoyancy length scale $l_{b} / D$ as a function of $R i$ in Figure 3-4 indicates that the dominant size of wave structures is the equivalent of 1 to 2 rotor diameters, which would place the corresponding dimensions of the secondary instabilities seen in Figures 2-3b and c equivalent to the rotor disk and smaller. The corresponding peak in the flapwise $L_{e q}$ response in this stability range confirms that the turbine is being affected.

An example of a response resulting from the ART rotor encountering a coherent turbulent structure in stable flow is shown in Figure 3-5. The background turbine layer $R i$ was +0.016 for this record and within the range discussed above. The excitation variables shown are the hubheight mean wind speed $U_{H}$, the three components of the Reynolds stresses, and TKE $(E)$. The response variables include displacements and angular rates measured by an inertial measurement unit (IMU) mounted atop the low-speed shaft forward support bearing and immediately behind the rotor. The displacements are the fore-aft $(x)$ and lateral $(y)$, and the angular rates are about the pitch and yaw axes. The zero-mean root flapwise bending completes the response. The plot shows the turbine encountering a coherent structure embedded in a gust structure near 500 seconds into the record that included a significant burst of TKE. The IMU displacement and angular rate traces clearly indicate strong peak-to-peak oscillatory (resonant) responses of more than $0.5 \mathrm{~mm}$ and 4 degrees, respectively, on the turbine nacelle over a period of about 10-12 seconds. At the same time, the blade root flapwise direction experienced a single load cycle of $560 \mathrm{kNm}$. Figure 3-6 shows the simultaneous measurements of vertical flux (transport of TKE across the turbine rotor disk). There is a strong downward flux of TKE at the rotor top (58 $\mathrm{m})$ that becomes smaller at the hub $(37 \mathrm{~m})$ and is largely damped out by the time it reaches the bottom of the rotor $(15 \mathrm{~m})$ because of vertical motion and turbulence damping as the result of increasing stability nearer to the ground. It is likely that the downward flux seen at the top of the rotor is the result of $\mathrm{KH}$ instability and breaking waves at or above the turbine. This is an example of the response of wind turbine rotors and structures to coherent turbulence and also demonstrates that other members of the turbine structure are involved and not only the blade roots.

In this section, we have used a selected subset of inflow parameters expressed as bulk statistics to relate the response of the root flapwise bending on the NWTC ART turbine. Using these statistics derived from more than 1500 10-minute records, we constructed a simple linear regression model in order to identify the inflow parameters with the highest correlation with root bending load response. We found that conditions within a narrow range of the dynamic stability in the atmospheric layer occupied by the wind turbine produced a large flapwise response in the blade root. Within this narrow stability range, large values of the standard deviation of the vertical wind speed and coherent turbulent energy accompanied the turbine response. We also found that the length scales of atmospheric wave motions in this stability range correspond to dimensions similar to that of the turbine rotor that would ensure the coupling of turbine higher order mode shapes with coherent turbulent structures of similar dimensions. We will use this simple regression model to estimate how the ART turbine would respond to conditions seen the LLLJP Site in southeastern Colorado. 
Table 3-1. NWTC/LIST Inflow and Response Summary by Dynamic Stability Category

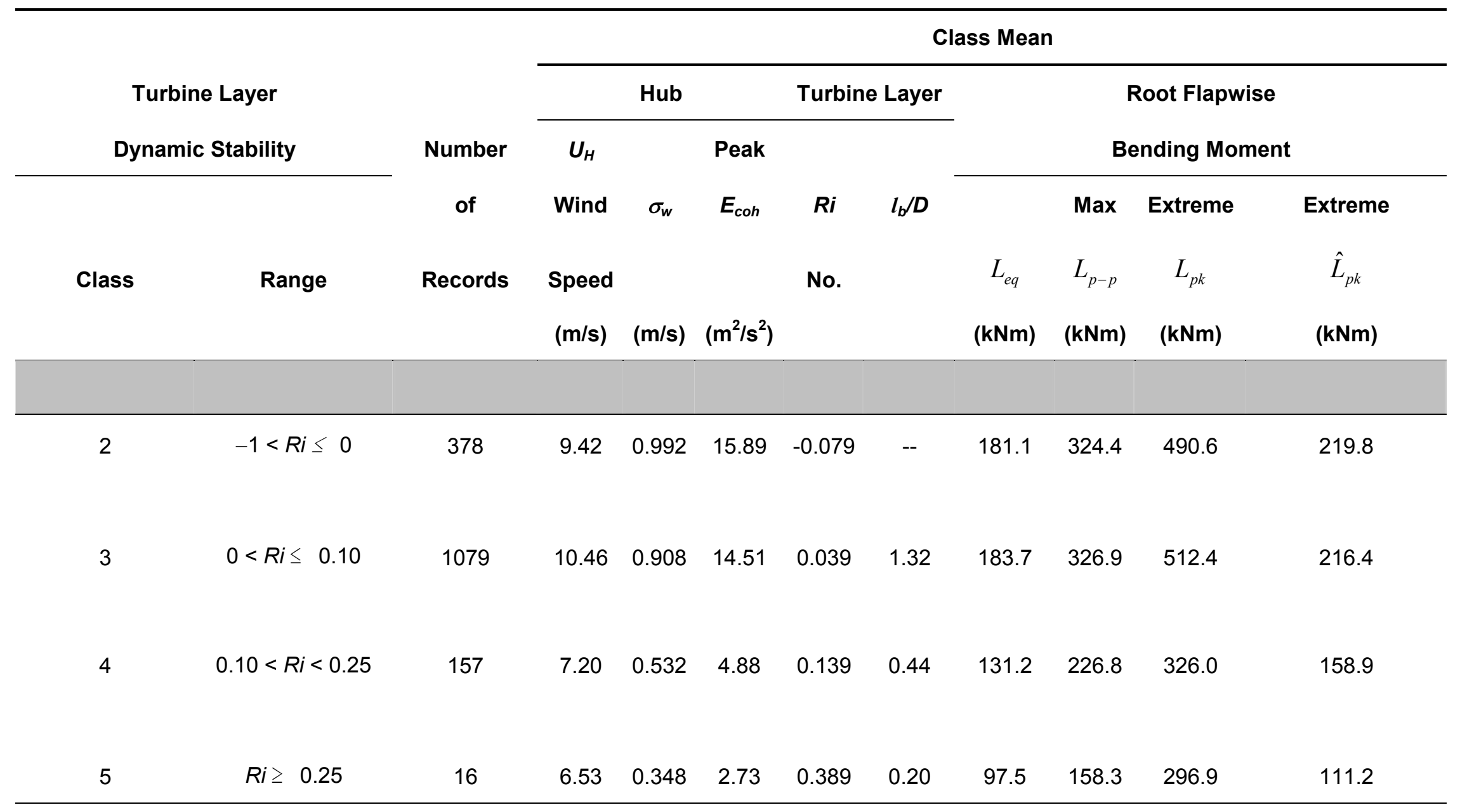


Table 3-2. Inflow Predictor Linear Correlation $\left(R_{i j}\right)$ Matrix

\begin{tabular}{cccccc}
\hline & $\boldsymbol{U}_{H}$ & $\sigma_{\mathrm{w}}$ & $\boldsymbol{R i}$ & $\boldsymbol{l}_{\boldsymbol{b}}$ & $\boldsymbol{E}_{\text {coh }}$ \\
\hline $\boldsymbol{U}_{\boldsymbol{H}}$ & & 0.541 & -0.113 & 0.170 & 0.418 \\
$\sigma_{\mathrm{w}}$ & 0.541 & & -0.421 & 0.268 & 0.822 \\
$\boldsymbol{R i}$ & -0.113 & -0.421 & & 0.092 & -0.244 \\
$\boldsymbol{l}_{\boldsymbol{b}}$ & 0.170 & 0.268 & 0.092 & & 0.242 \\
$\boldsymbol{E}_{\text {coh }}$ & 0.418 & 0.822 & -0.244 & 0.242 & \\
\hline
\end{tabular}

Table 3-3. Inflow/Response Variable Linear $\left(R_{i j}\right)$ Correlations

\begin{tabular}{cccccc}
\hline & $\boldsymbol{U}_{\boldsymbol{H}}$ & $\sigma_{\mathbf{w}}$ & $\boldsymbol{R i}$ & $\boldsymbol{l}_{\boldsymbol{b}}$ & $\boldsymbol{E}_{\text {coh }}$ \\
\hline$L_{e q}$ & 0.615 & 0.867 & -0.283 & 0.297 & 0.717 \\
$L_{p-p}$ & 0.584 & 0.835 & -0.283 & 0.281 & 0.693 \\
$L_{p k}$ & 0.718 & 0.749 & -0.249 & 0.256 & 0.614 \\
$\hat{L}_{p k}$ & 0.348 & 0.746 & -0.274 & 0.239 & 0.684 \\
\hline
\end{tabular}

Table 3-4. Inflow Parameter Sensitivity

\begin{tabular}{cccccc}
\hline & $\boldsymbol{U}_{\boldsymbol{H}}$ & $\boldsymbol{\sigma}_{\mathrm{w}}$ & $\boldsymbol{R i}$ & $\boldsymbol{l}_{\boldsymbol{b}}$ & $\boldsymbol{E}_{\text {coh }}$ \\
\hline$L_{e q}$ & $\checkmark$ & $\checkmark$ & $\checkmark$ & & \\
\hline$L_{p-p}$ & $\checkmark$ & $\checkmark$ & $\checkmark$ & & \\
\hline$L_{p k}$ & $\checkmark$ & $\checkmark$ & & & \\
\hline$\hat{L}_{p k}$ & & $\checkmark$ & & $\checkmark$ & $\checkmark$ \\
\hline
\end{tabular}


Table 3-5. Regression Results Summary

\begin{tabular}{|c|c|c|c|c|}
\hline & F-value & $r^{2}$ & std err of regr & \\
\hline & & & $(\mathrm{kNm})$ & \\
\hline$L_{e q}$ & 1993.92 & 0.786 & 17.71 & \\
\hline$L_{p-p}$ & 1434.68 & 0.725 & 39.55 & \\
\hline$L_{p k}$ & 1901.62 & 0.699 & 49.07 & \\
\hline \multirow[t]{2}{*}{$\hat{L}_{p k}$} & 730.19 & 0.573 & 32.73 & \\
\hline & t-value & $\begin{array}{c}\text { Significance } \\
\text { Level }\end{array}$ & $\begin{array}{c}\text { Fitted } \\
\text { Coefficients }\end{array}$ & $\begin{array}{l}\text { Std } \\
\text { Dev }\end{array}$ \\
\hline \multicolumn{5}{|l|}{$L_{e q}$} \\
\hline$U_{H}$ & 14.115 & 0.0001 & 2.786601 & 0.197 \\
\hline$\sigma_{w}$ & 52.433 & 0.0001 & 114.124329 & 2.177 \\
\hline$R i$ & 5.669 & 0.0001 & 33.136495 & 5.845 \\
\hline & & & & \\
\hline \multicolumn{5}{|l|}{$L_{p-p}$} \\
\hline$U_{H}$ & 11.318 & 0.0001 & 4.991084 & 0.441 \\
\hline$\sigma_{w}$ & 44.701 & 0.0001 & 217.330117 & 4.862 \\
\hline$R i$ & 4.111 & 0.0001 & 53.679858 & 13.057 \\
\hline & & & & \\
\hline \multicolumn{5}{|l|}{$L_{p k}$} \\
\hline$U_{H}$ & 27.442 & 0.0001 & 14.841126 & 0.541 \\
\hline$\sigma_{w}$ & 31.592 & 0.0001 & 171.921519 & 5.442 \\
\hline & & & & \\
\hline \multicolumn{5}{|l|}{$\hat{L}_{p k}$} \\
\hline$\sigma_{w}$ & 19.491 & 0.0001 & 105.278001 & 5.401 \\
\hline$l_{b}$ & 2.218 & 0.02672 & 0.03696 & 0.017 \\
\hline$E_{c o h}$ & 7.626 & 0.0001 & 1.087368 & 0.143 \\
\hline
\end{tabular}




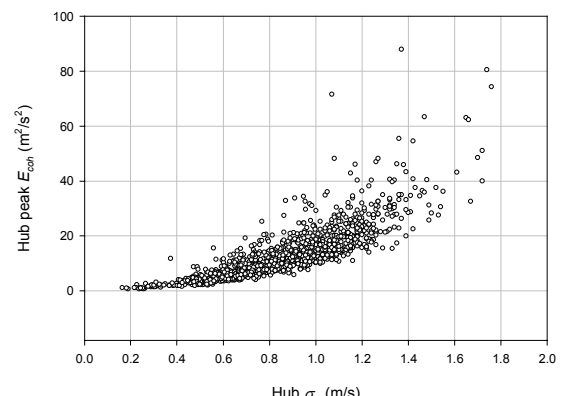

Hub peak $E_{c o h} \mathrm{vs} \sigma_{w}$

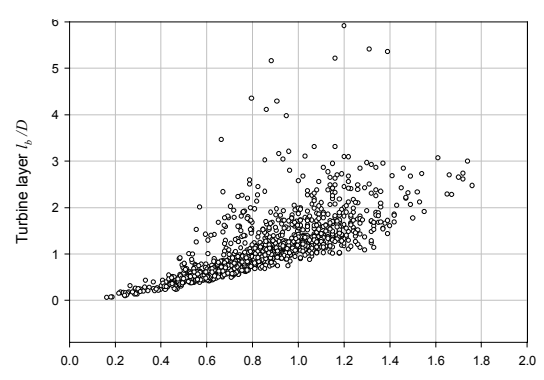

Turbine layer $l_{b} / D$ vs Hub $\sigma_{w}$

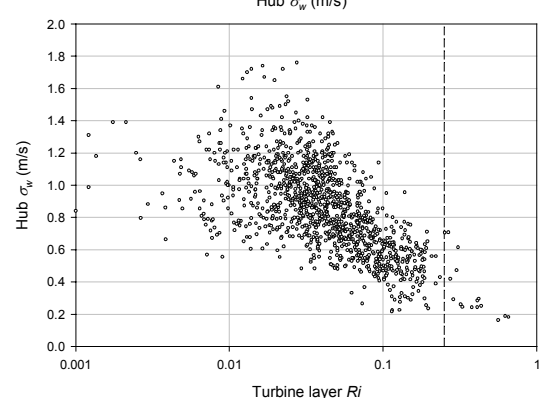

Hub $\sigma_{w}$ vs Turbine layer $R i$

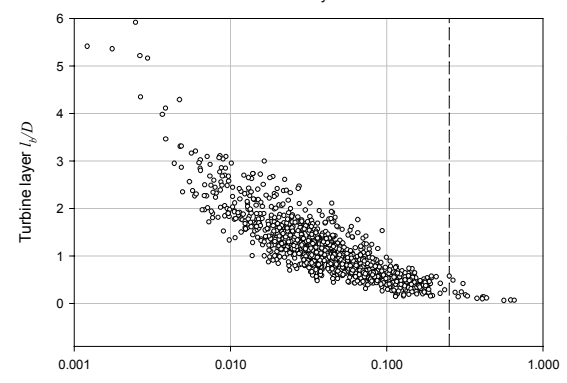

Turbine layer $l_{b} / D$ vs Turbine layer $R i$

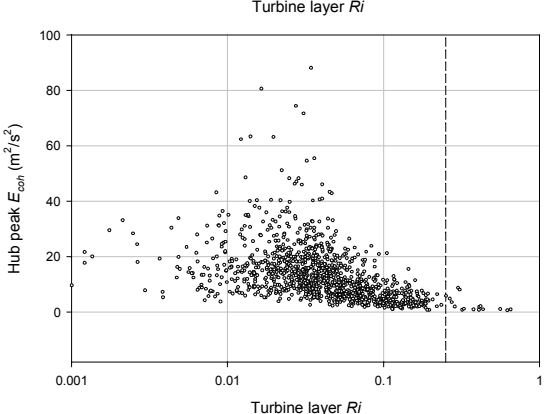

Hub peak $E_{c o h}$ vs Turbine layer $R i$

Figure 3-1. Variation of observed inflow parameters during NWTC/LIST experiment October 28, 2000, to May 17, 2001 


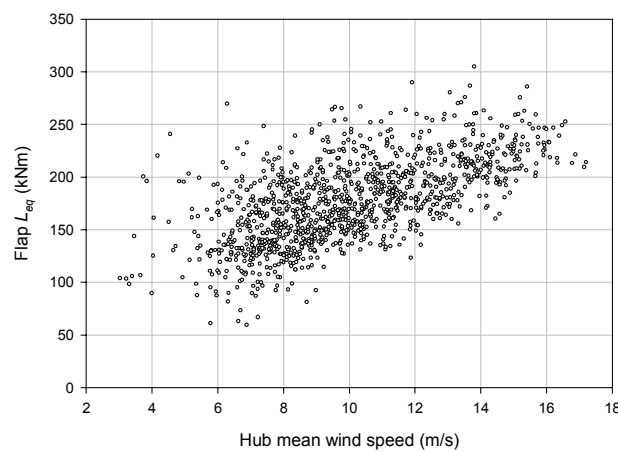

Flap $L_{e q}$ vs Hub $U_{H}$

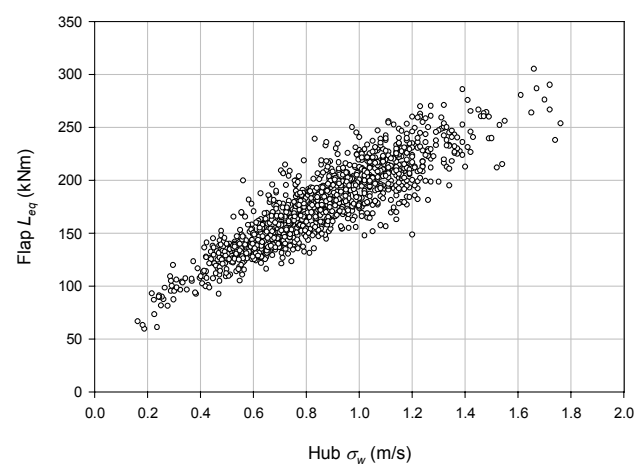

Flap $L_{e q}$ vs Hub $\sigma_{w}$

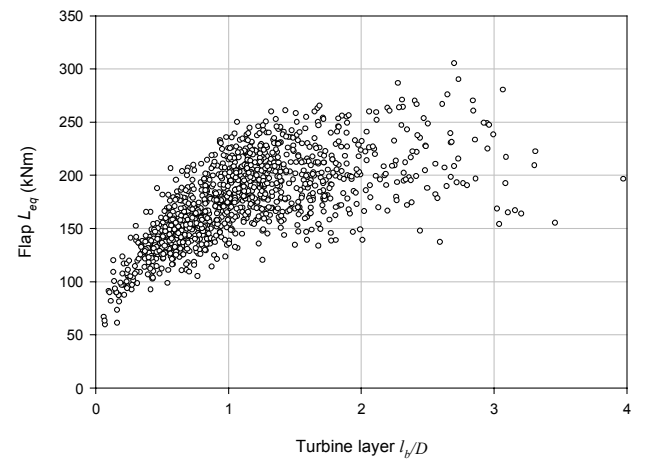

Flap $L_{e q}$ vs Turbine layer $l_{b} / D$

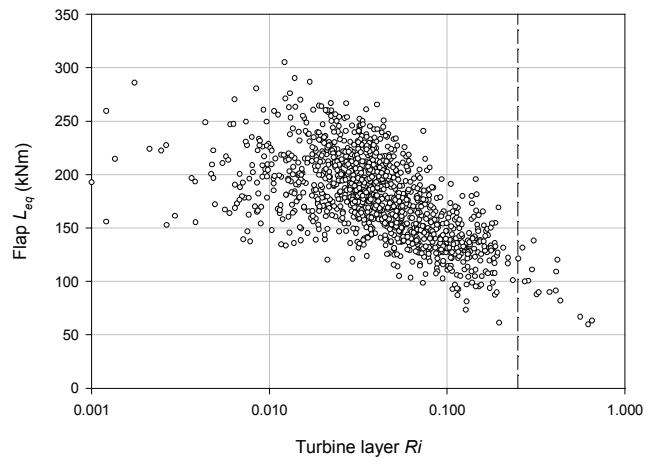

Flap $L_{e q}$ vs Turbine layer $R i$

Figure 3-2. Variation of observed flapwise $L_{e q}$ versus inflow parameters during NWTC/LIST experiment 

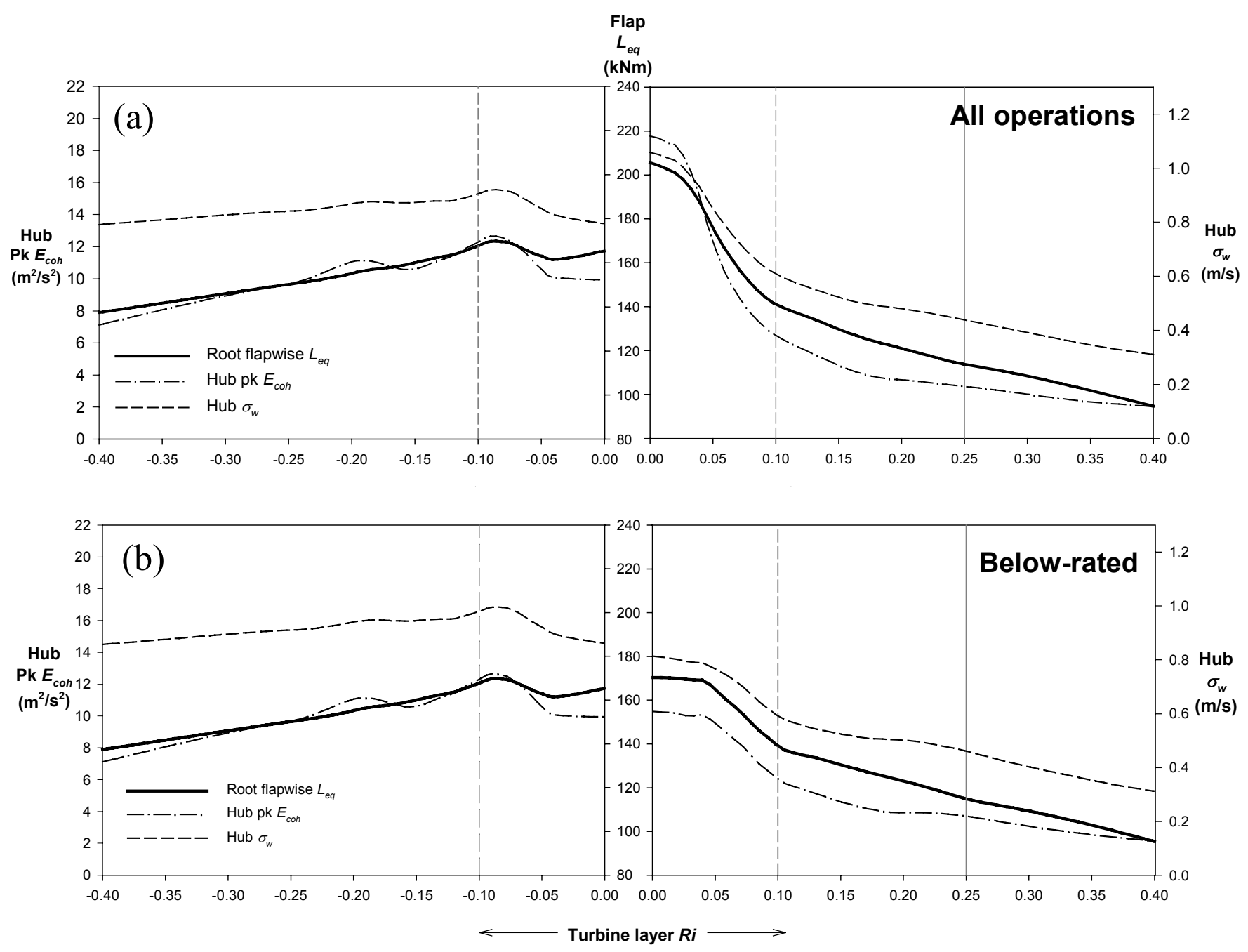

Figure 3-3. Variation of ART turbine root flapwise equivalent loads $L_{e q}$ and hub-height vertical wind speed standard deviation $\sigma_{w}$ and peak coherent turbulent kinetic energy $E_{c o h}$ as a function of the turbine-layer gradient Richardson number Ri for NWTC/LIST experiment; (a) all operations, (b) below-rated operations only 


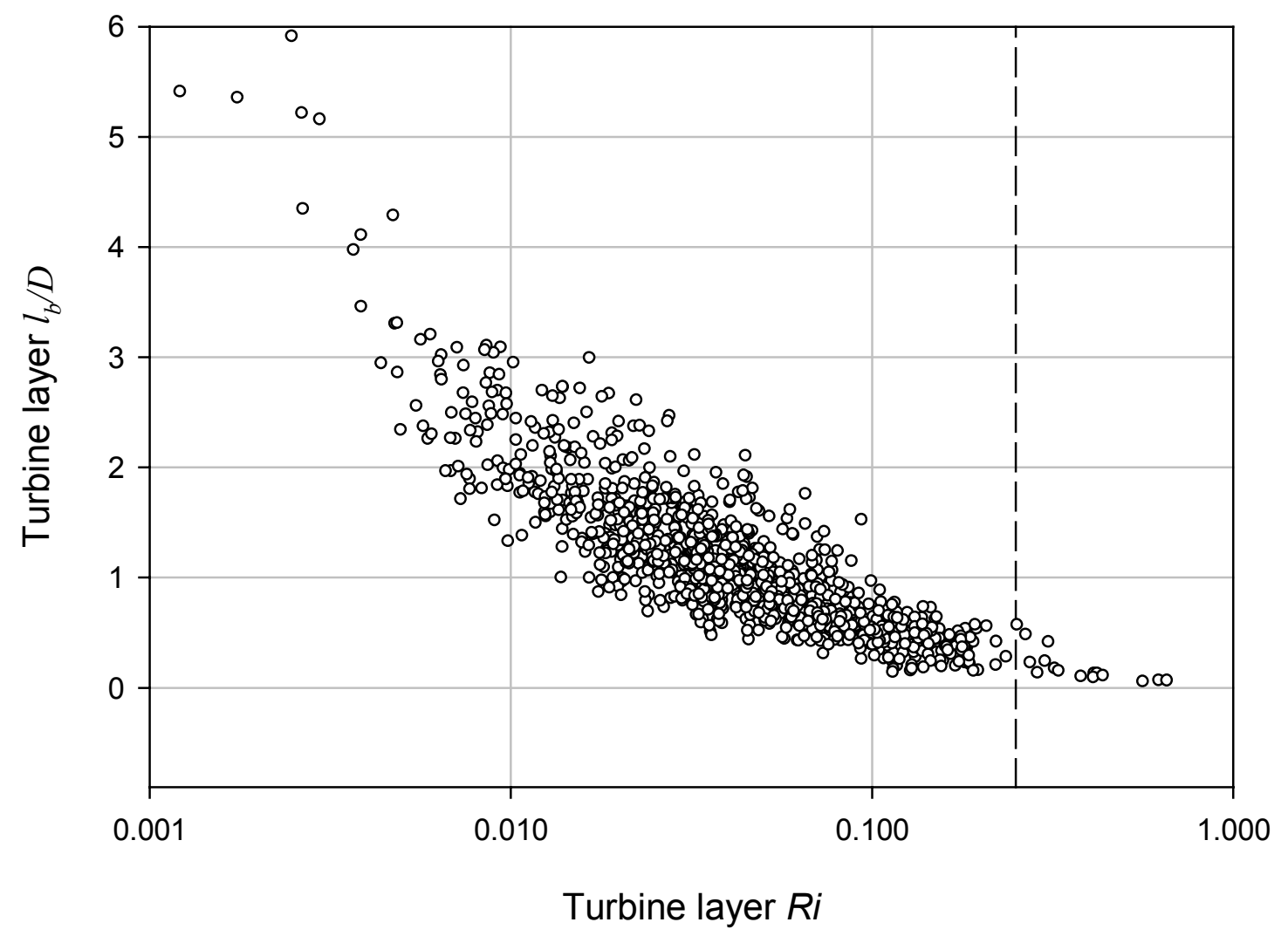

Figure 3-4. Variation of turbine-layer buoyancy length scale $I_{b}$ normalized by the ART turbine rotor diameter $D\left(I_{b} / D\right)$ for NWTC/LIST experiment 

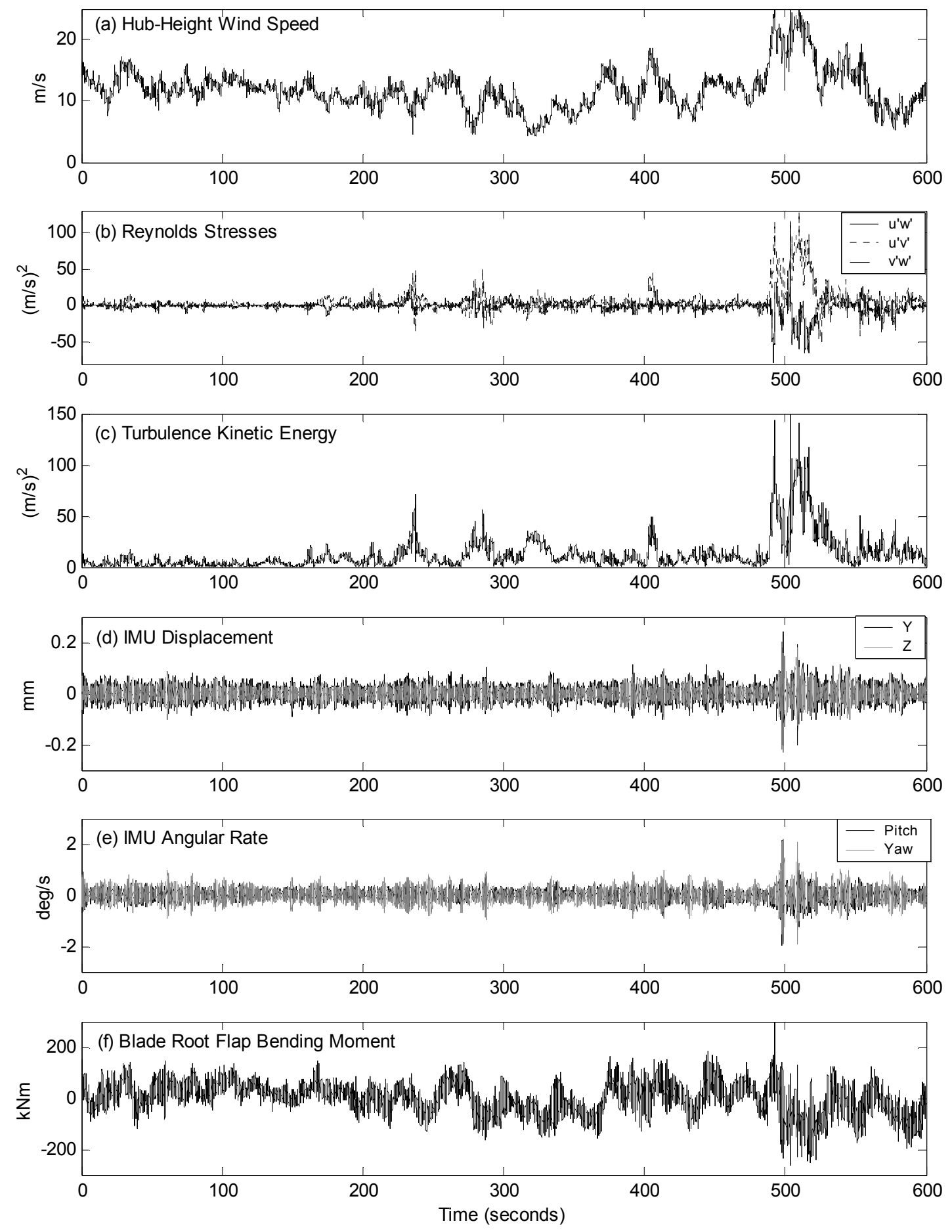

Figure 3-5. Time history example of a coherent turbulent structure in a nocturnal stably stratified flow and the corresponding response of the ART turbine 

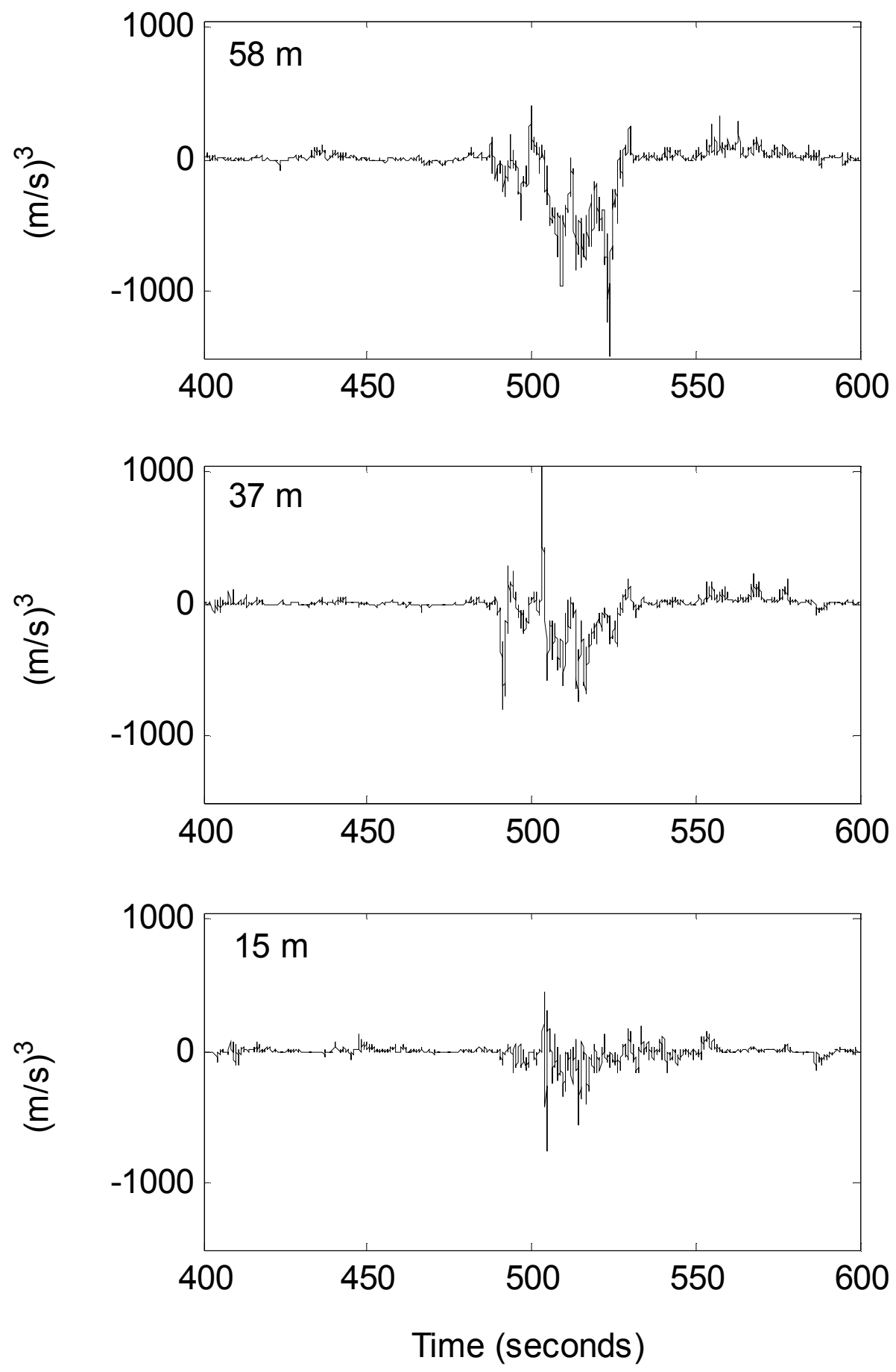

Figure 3-6. Example of variation of the downward flux of turbulent kinetic energy $E$ recorded by the NWTC/LIST upwind array of sonic anemometers 


\section{The Lamar Low-Level Jet Program}

The Lamar Low-Level Jet Program (LLLJP) was established as a joint effort between the U.S. Department of Energy (DOE), NREL's National Wind Technology Center, and General Electric Wind Energy. The objective of this program was to characterize the turbulence environment at a representative Great Plains wind resource site at heights in the atmosphere where new designs of low-wind speed turbines (LWST) will be installed over the next few years. Of particular concern was the nocturnal low-level jet stream that forms frequently over the Great Plains, particularly during the warmer months. It is a program objective to develop an understanding of the role of the jet in producing lower level shear and turbulence where wind turbines will be operating. The information gathered is being used to improve the turbulence simulation codes applied as an important tool in the turbine design process.

To acquire the necessary information to meet the program goals, General Electric Wind Energy (GE Wind) installed a 120-m (400-ft) meteorological tower at the site of a planned wind farm development south of Lamar, Colorado. NREL instrumented that tower with several levels of sensitive instrumentation to measure the turbulence environment. In addition, NREL installed an acoustic wind profiler (SODAR) nearby to locate and quantify low-level jets seen over the site and correlate that information with turbulence data collected on the tower. A collaborative program with the National Oceanic and Atmospheric Administration's Environmental Research Laboratory (NOAA/ETL) has been established and a brief field measurement program at the LLLJP Site was executed in early September 2003. This effort employed their high-resolution Doppler LIDAR (HRDL) in conjunction with the tower-based measurements and the SODAR.

\subsection{Instrumentation and Data Collection}

The LLLJP site is located about $32 \mathrm{~km}$ (20 miles) south of Lamar, Colorado, on the Emick Ranch Property in southern Prowers County. It is situated on a high plateau south of the Arkansas River Basin. Locally, the terrain is flat and homogenous, but with more complex elements to the west and north. The geographical coordinates of the tower are $37^{\circ} 40.099^{\prime} \mathrm{N}$ and $102^{\circ} 39.825^{\prime} \mathrm{W}$. Its base is at an elevation of $1357 \mathrm{~m}(4451 \mathrm{ft})$ above mean sea level. The tower is a custom triangular-guyed design built by the Rohn Company to NREL and GE Wind specifications to provide a high degree of torsional stiffness. This was necessary to minimize the lateral movements of the instrument support arms that extend more than $5 \mathrm{~m}$ (18 ft) away from the tower base. The tower sides are $1.04 \mathrm{~m} \mathrm{(41} \mathrm{in.)} \mathrm{wide} \mathrm{at} \mathrm{the} \mathrm{base.} \mathrm{The}$

tower is the tallest structure in the vicinity and, therefore, susceptible to lightning strikes and corona discharges. A grounding grid was installed underneath the site, connecting the tower base and all guy anchors and to which all the electrical service is referenced. Figure 4-1 pictures the tower installation.

\subsubsection{Tower-based Instrumentation}

The tower-mounted instrumentation included three-axis sonic anemometers installed at the 54-, 67-, 85-, and 116-m heights. The sonic anemometers were attached to custom-designed support arms (designed and constructed by Martin/Martin Consulting Engineers) that allow the placement of the sensing heads five tower widths $(\sim 5 \mathrm{~m})$ away from the tower itself to minimize local flow distortion caused by the presence of the structure. The support arms 
were designed to damp all motions below $10 \mathrm{~Hz}$, the upper limit of the velocity frequency range of interest. The support arms were mounted facing toward $300^{\circ}$ with respect to True North to capture the prevailing winds from both summer and winter with minimal flow distortion from the tower. Figure 4-2 provides a close-up view of the instrument arm and sonic installation. The anemometers were supplied by Applied Technologies, Inc. (ATI) Model SATI $/ 3 \mathrm{~K}$. These instruments had a $15-\mathrm{cm}$ path length and provided velocity measurements from three orthogonal axes and sonic air temperature at a rate of 20 samples per second and a resolution of $1 \mathrm{~cm} / \mathrm{s}$. A three-axis, force-balance accelerometer was attached inboard of the sonic anemometer at the $85-\mathrm{m}$ level. This accelerometer is used for detecting large lateral movements of the support arm due to torsional movements of the tower that are sensed incorrectly as wind velocities. A dynamic analysis of the tower system performed when the support arms were being designed suggested that significant motions of the tower itself could occasionally be present under gust loading because there were no stabilizing guy wires nearby.

Wind speed and direction are measured using Met One WS-201 cup anemometers and highly damped direction vanes that were mounted at the 3-, 52-, and 113-m levels. Air temperatures are measured at heights of 3,52, and $113 \mathrm{~m}$, with precision temperature differences measured between 52 and $83 \mathrm{~m}$ and between 83 and $113 \mathrm{~m}$. All temperature measurements are acquired with four-wire platinum (Pt100) resistance temperature detectors (RTD's) placed within mechanically aspirated radiation shields. Dew-point temperatures are measured at 3, 52, 85, and $113 \mathrm{~m}$ using Met One Model DP200B Lithium-Chloride (LiCl)/RTD dew-point hygrometers. Barometric pressure is measured at a height of $3 \mathrm{~m}$ using a Vaisala PTB100 pressure transducer that is connected to an external omni-directional sampling head on the exterior of the building.

\subsubsection{Acoustic Wind Profiler (SODAR)}

An acoustic wind profiler (SODAR) was installed in late May 2002 at location southeast of the tower base. This Scintec Model MFAS mid-range sodar had a vertical measurement range from 20 to $1000 \mathrm{~m}$ in $10-\mathrm{m}$ increments. It provided wind profiles of the horizontal wind speed and direction, the north-south, and east-west components, and the vertical component at the 10-m vertical resolution. This instrument utilizes a phased-array antenna that can provide nine electronically steerable beams emitting up to 10 frequencies (Figure 43 ). The antenna is installed within an octagonal acoustic enclosure designed to reduce environmental noise at the antenna itself. The system works in conjunction with a personal computer to provide online data processing and recording.

The SODAR antenna is located $109 \mathrm{~m}(358 \mathrm{ft})$ to the southeast of the tower base. This location was chosen to be as close as possible to the tower in order to obtain a better comparison of profiler-derived winds with those directly measured by instruments on the tower. Using the antenna radiation pattern furnished by the manufacturer, we chose the location shown in Figure 4-4 so that the main and principal side lobes of acoustic energy that radiated in the cardinal directions would not illuminate the tower.

In December 2002, a Scintec representative visited the site and agreed with our location of the antenna. 


\subsubsection{Data Acquisition}

The tower-based measurements were collected using two recording systems. A subset of the measurements (not including the sonic anemometers) were collected continuously as statistics (means and standard deviations for wind-related parameters) over a 5-minute period by a Campbell-Scientific 23X data logger. This information was stored and then transferred to NREL every 3 hours via landline. In addition to the meteorological data, parameters related to site security, the status of electrical service (commercial power or an uninterruptible backup), and equipment and building temperatures were also included for remote monitoring purposes. The information received at NREL was placed on an internal web page that provides both text and graphical data capabilities.

A second system was employed to collect the high-resolution sonic information in addition to the other tower data. This system is PC-based and uses National Instruments (NI) interfacing modules and its LabVIEW ${ }^{\odot}$ operating software to collect the sonic and other tower data. Data were collected at 20 samples per second on all data channels from 16 to $08 \mathrm{~h}$ local standard time each day to observe conditions primarily in the nocturnal boundary layer. The data is stored locally on both multiple hard disk drives and a removable magneto-optical disk. The latter was removed and shipped to NREL once a week. Between 08 and $15 \mathrm{~h}$, some or all of the most recent data collected was transferred to NREL via an Internet satellite link. This allows quality control examination within a day or so after the data is collected to identify any issues that may be developing.

We operated the tower low-speed data collection system continuously from October 5, 2001, through September 16, 2003. The high-speed system was operated for a period of 1 year beginning in late March 2002 and ending at the beginning of April 2003 and again briefly during the measurements with the NOAA Lidar. We have collected more than 30,000 10minute records that monitored the turbulence environment of the nocturnal boundary layer over this period

The SODAR system operates with its own dedicated PC, but has recording capabilities similar to the high-speed tower system (i.e., multiple hard disks and a removable magnetooptical drive). As a precaution, we record both the processed and raw spectral data. By saving the later, we can perform a certain level of re-processing using later software releases from the manufacturer. While we cannot change parameters such as the number of emitted beams, their frequencies, pulse lengths, and integration periods, we can take advantage of improved signal processing capabilities available with later software releases. The negative side is that the data storage requirements are much larger, but the ability to apply better software routines to existing data sets is a distinct advantage.

We employed a number of system configurations to achieve better results. The system operated more or less continuously from the end of May 2002 through early November 2003, when it was shut down and returned to the factory in Germany to locate and solve an internal power-line noise issue. During this period, we used a 10-minute integration period coupled with a measurement height range from 40 to $500 \mathrm{~m}$. We tried various approaches to improve the data capture capabilities of the system, including ranging from multiple frequencies and the maximum number of emitted beams (9) to a single frequency using emissions from only the west, south, and vertical beams (the minimum necessary to obtain horizontal and vertical 
wind speeds). We also experimented with varying the pulse length and, to some extent, its repetition frequency. In the end, we found that we achieved more or less the best combination by using a long (100-ms) $2022-\mathrm{Hz}$ pulse toward the west, south (away from the nearby tower), and vertical directions. However, as the weather cooled during the fall, this approach did not work as well. In spite of this, the system has obtained excellent data regarding the height of the low-level jet and the vertical shear below. We also have the tower measurements for those periods and three examples of such conditions will be discussed in a later section.

We have recently been working closely with the manufacturer to help improve the capabilities of this instrument. A considerable amount of progress has been accomplished over the past 2 or 3 months principally through improved acoustic signal processing algorithms used to extract the wind speeds from the backscattered, Doppler-shifted acoustic energy. Our plan is to use the improved software to re-process the raw data collected last year in order to obtain the maximum information possible about the characteristics of the LLJ.

\subsection{Known Data Limitations}

In general, the tower-based measurement systems functioned very well over the past 1-1/2 years. As discussed above, the SODAR was another situation entirely. We will discuss known limitations for each below.

\subsubsection{Tower-based Measurement Limitations}

Problems associated with the tower measurement data collection fall into two categories: (1) periods of temporary data loss as the result of weather events or system component failure; and (2) systemic problems that cannot be removed and that, therefore, in some way degrade the final results. Fortunately, we did not suffer extensive data losses with events in the first category, and we know of only one issue in the second at the present time. It is certainly possible that as the collected data are processed additional issues may arise.

\subsubsection{Temporary tower data losses}

We knew that lightning and corona discharge would be a potential problem given the height of the tower and its isolation from other tall nearby structures. To minimize damage from lightning and corona as much as possible, we installed a grounding system to which the tower and its anchors, the data building, the SODAR, and the electrical power system were all attached. All signal wires entering the data building from the tower, SODAR, and telephone lines pass through one or more lightning protection circuits.

In June 2002 we noticed the appearance of what appeared to be high-frequency noise in the sonic anemometer velocity and temperature measurements. An analysis revealed that this noise appeared during periods of nearby thunderstorm activity. The problem was most acute on the topmost sonic anemometer, though the lower ones exhibited it on occasion. We concluded that because the highest anemometer was located so far away from the tower, it extended beyond the so-called "cone of protection" afforded by the cone-shaped zone with the lightning rod at the tower top as the vertex. To counter this situation, we designed a small rigid copper corona point that could be mounted to the anemometer in such a way as to 
not induce any appreciable flow distortion in the measuring paths. This device was attached to a large-gage copper wire that was then connected to the tower main grounding system. As a further precaution, we mounted aircraft-type static discharge devices on the instrument support arms to relieve the local field if it should build up. We found that these devices definitely helped reduce the noise in the sonic data from corona discharges except in only the most violent thunderstorms.

We suffered only two major lightning episodes in which some damage occurred. The first occurred in early August 2002 when the site was involved in a continuous lightning storm that the rancher describes as lasting more than 6 hours. He also witnessed the tower suffer direct strikes at least three times. In this storm, we lost two of our temperature measurements and the topmost sonic anemometer. The SODAR stopped functioning, but it only required being re-initialized. An examination of the tower revealed that the lightning strike had missed the lightning rod and hit a nearby climbing apparatus, causing the charge not to completely travel through the grounding system, but through the structure itself. We have since doubled the height of the tower-top lightning rod.

An examination of the topmost sonic anemometer revealed that the tip of the corona point had been completely melted, indicating that it did its job. The sonic anemometer had not been damaged and, like the SODAR, only needed to have its digital circuitry reset.

None of the temperature probes or wiring was damaged on the tower, and the source of the problem was found to be a particular integrated circuit device in each of the temperature translator units. The data outage lasted for only a few days. We recently suffered an outage from a similar storm, but only the same integrated circuits were involved. In general, we believe the precautions we have taken have held lightning and corona discharge damage to a minimum given the number of thunderstorms passing over the site during the last year and a half.

The only other major data outage (other than have a sonic anemometer fail and being without it for a few days) has been when the radiation shield was loosened by high winds in late May and slipped down, exposing the temperature measuring probes to the direct sun.

Unfortunately, we did not identify this situation until early July because there was an almost constant daytime cloud cover and frequent storminess before that. Excessive temperatures were then noticed on days when only a few clouds were present. The loss of this temperature measurement prevents us from calculating the stability in the layers between 52 and $113 \mathrm{~m}$ and 85 and $113 \mathrm{~m}$. Thus temperatures and stability are only available after sunset and before sunrise (nominally 19 to $05 \mathrm{~h}$ local standard time) for this approximately 6 -week period. The loss related to the strike in June 2003 lasted only a couple of days however.

\subsubsection{Systemic tower measurement issues}

An examination of all of the sonic anemometer velocity signals (the vertical velocity signal from the 85-m level in particular) revealed what appeared to be high-frequency noise that was not related to atmospheric electrical activity. A further examination showed a similar situation in some of the signals derived from the close-by accelerometer, suggesting that the problem was not electrical but mechanical. A spectral analysis of the accelerometer signals revealed that the support arm was oscillating at about $8.5 \mathrm{~Hz}$, primarily in the lateral and vertical directions. A cross-spectral analysis between the accelerometer signals and the sonic vertical velocity signal confirmed that the "noise" velocities had the same frequency as the 
support arm, indicating that the movement of the support was inducing a false velocity in to the wind component signals.

Because $8.5 \mathrm{~Hz}$ is much higher than would be expected from natural frequencies associated with the tower and the instrument arm was damped at this frequency by design it is not certain what the source of the vibration was. A review of the structural elements comprising the tower and their dimensions revealed that it was constructed wholly of circular elements or tubes (i.e., large circular vertical apex legs and smaller circular cross bracing members).

Because these members were circular, the possibility of Aeolian vortex shedding was considered. The vertical main structural members have a diameter of $9 \mathrm{~cm}(3.5 \mathrm{in}$.) near the base of the tower and $8 \mathrm{~cm}$ ( $3 \mathrm{in}$.) at the top. The cross-members have a diameter of $4 \mathrm{~cm}$ (1.5 in.) At an incident wind speed of $10 \mathrm{~m} / \mathrm{s}$, the vortex shedding frequency is about $4.5 \mathrm{~Hz}$ for the large leg and $8.5 \mathrm{~Hz}$ for the cross member. This seemed to be the source of the problem (i.e., the tower was vibrating at $8.5 \mathrm{~Hz}$ when the wind was near $10 \mathrm{~m} / \mathrm{s}$ [which was confirmed by our tower data] causing the observed induced velocities in the sonic-measured wind speeds). In September, the sonic anemometer at the $85-\mathrm{m}$ level began producing large random noise spikes. Subsequent repair efforts could not find the problem; the whole internal electronics package was replaced though it could not be confirmed the problem had been solved.

We decided to make sure the instrument was actually repaired before using it to replace another unit that had failed. We decided to mount the anemometer and its support arm on a computer-controlled vibration shaker to verify that the repair was successful and thus confirm the high-frequency velocity measurements we had seen in the data collected on the tower. We mounted the anemometer cantilevered from its support arm as it was on the tower and excited it with a constant-acceleration 5- to $-1000-\mathrm{Hz}$ sine-wave sweep. We found the actual resonance of anemometer and support was $11 \mathrm{~Hz}$ in the lateral direction and slightly lower in the vertical. With this knowledge, it became clear that $8.5-\mathrm{Hz}$ noise signals in our data collected from the tower accelerometer and sonic anemometer were the result of the 11 $\mathrm{Hz}$ vibration motions being aliased or folded back as a result of the $20 \mathrm{-Hz}$ sampling rate (i.e., the 11-Hz oscillations were being under-sampled and appeared as $8.5-\mathrm{Hz}$ in the data). Internally, the sonic velocity signals were sampled at $200 \mathrm{~Hz}$ and then block averaged 10:1 to $20 \mathrm{~Hz}$. The frequency $(H(f))$ transfer function of this low-pass filter is plotted in Figure 4-5 against both a linear and logarithmic frequency axes. The Nyquist (folding frequency $f_{N}$ ) of $10 \mathrm{~Hz}$ and the ATI mounting arm are indicated. Thus, signal energy to the right of (higher frequency than) $f_{N}$ will be aliased or folded back and appear at a lower frequency in the recorded velocity time histories.

We also learned that the $11-\mathrm{Hz}$ resonance was relatively lightly damped and as little as a 0.25 -g excitation can produce large displacements of the sonic measuring head, which accounted for the motion-induced velocities seen in the sonic anemometer output signals. Figure 4-6 shows the relationship between the wind velocity and the vortex shedding frequency for both the tower large vertical legs and the cross members. It indicates that a wind speed in the neighborhood of $12 \mathrm{~m} / \mathrm{s}$ will produce a vortex shedding frequency of $11 \mathrm{~Hz}$ that appears in our data as a peak at $8.5 \mathrm{~Hz}$. This is a very common wind speed at the $85-\mathrm{m}$ level. The sonic anemometer manufacturer believes that the vibration likely caused a micro crack to develop in the internal circuit board and that, in turn, was responsible for the noise 
spikes seen in the data. Our only recourse to avoid this high-frequency noise is to remove frequency components in the sonic velocities signals above at least $7 \mathrm{~Hz}$ by low-pass filtering during final post-processing.

\subsubsection{SODAR Measurement Limitations}

How successful we will be in obtaining useable wind profile and other parameters that can be derived from the SODAR measurements collected between late May and early November will depend on the ability of the latest available Scintec signal processing software to handle the range of configurations used. Early indications are that this capability has been significantly improved, particularly with respect to the detection and elimination of fixed echoes being reflected from the nearby tower. However, it remains to be seen how effective the software will be when only long period $(100 \mathrm{~ms})$, single-frequency pulse was emitted in the west, south, and vertical directions. We used this arrangement for an extended period of time in late summer and early fall. Thus, our ability to fully characterize the presence and evolution of LLJs will depend to how much information can be obtained from reprocessing of the collected raw spectral data using the latest software.

The SODAR was shut down in early November because it could not produce usable results. A visit from a Scintec representative in early December found that there was $60-\mathrm{Hz}$ noise contaminating the backscattered signals. The source of the noise was found to be within the electronics incorporated in the antenna itself. Furthermore, it was determined that even with emitting energy away from the nearby tower (from the west, south, and vertical beams) there were fixed echo reflections being received because the antenna side lobes and the signal processing software was not adequately detecting and removing them. It was decided to return the system to Germany because special equipment would be required to track down and eliminate the power line noise contamination. The unit was not returned to us until May 2003, at which time it was re-installed at the LLLJP Site. While the revised software furnished at the time of re-installation significantly improved the fixed echo problem, a number of other issues remained. Working in coordination with the manufacturer, we have since tracked down this problem to signal processing issues. Significant progress has been achieved over the past 2 or 3 months.

The loss of the SODAR from November through April will unfortunately limit our ability to more fully understand the cold-season LLJ formation and wind-profile development. We do have the high-speed, detailed turbulence measurements from the tower-based instruments providing an incomplete picture.

\subsection{Future Measurements}

During the first 2 weeks of September 2003, we worked with the NOAA Environmental Technology Laboratory (ETL) to make a series of measurements with their High-Resolution Doppler LIDAR (HRDL) at the LLLJP Site. The objectives of this work include the following:

- Determining the usefulness of Doppler LIDAR for wind-energy resource assessment at heights up to $200 \mathrm{~m}$ above ground level 
- Determining the usefulness of Doppler LIDAR for characterizing turbulence events and for diagnosing periods when turbulence potentially damaging to wind turbines is likely to occur

- Investigating the horizontal distribution and dimensions of turbulence or wave packets in the nocturnal stable boundary layer

- Determining what information may be obtained by pointing the LIDAR beam into the wind in a similar manner in which a forward-looking LIDAR would be used with a wind turbine.

The Doppler LIDAR wind data will be compared with that measured on the 120-m tower and by the SODAR. We hope that the results of this effort will provide additional information about the turbulence conditions surrounding the presence of a low-level jet stream above the range of the tower measurements. Such information is needed as turbine rotors continue to rise higher into the boundary layer.

Some examples of observations collected by the NOAA HRDL during the CASES-99 Project (1999 Cooperative Atmospheric Surface Exchange Study) in southern Kansas are presented in Figure 4-7. Figure 4-7a displays a 30-s record associated with the day-night transition. Here the wind was blowing toward the LIDAR as indicated by the maximum of $-12 \mathrm{~m} / \mathrm{s}$ (green). Convective plumes of lower-speed air (yellow-brown) can be seen rising from the ground with fingers of higher speed (blue and dark blue) and probably more turbulent air reaching downward. The formation of a weak low-level jet at a height of about $200 \mathrm{~m}$ is shown in Figure 4-7b. Finally, Figure 4-7c displays a layer of gravity-shear waves (likely the result of some form of shear instability, KH perhaps) forming just below a LLJ at a height of about $50 \mathrm{~m}$. In this presentation, the wind was blowing away from the LIDAR and the highest wind speeds are now shown in red $(+10 \mathrm{~m} / \mathrm{s})$. Questions we would like to answer include:

- Do the wave motions extend laterally and, if so, how far?

- Do they cover the area of a large wind farm or more?

- Is it possible to use a single point measurement in or near a large wind farm as a detector of coherent turbulence activity? 


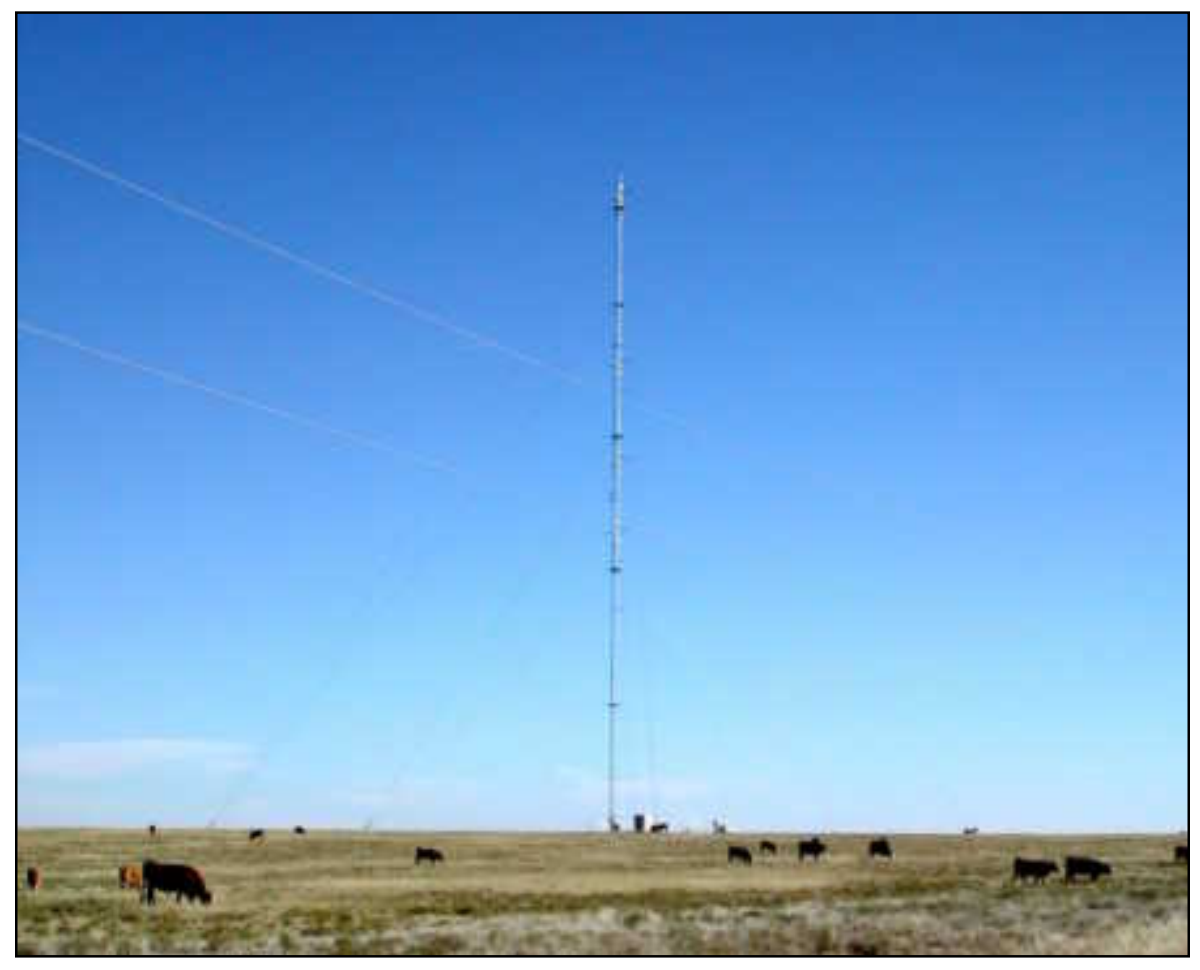

Figure 4-1. GE Wind 120-m met tower - southeast Colorado (LLLJP Site)

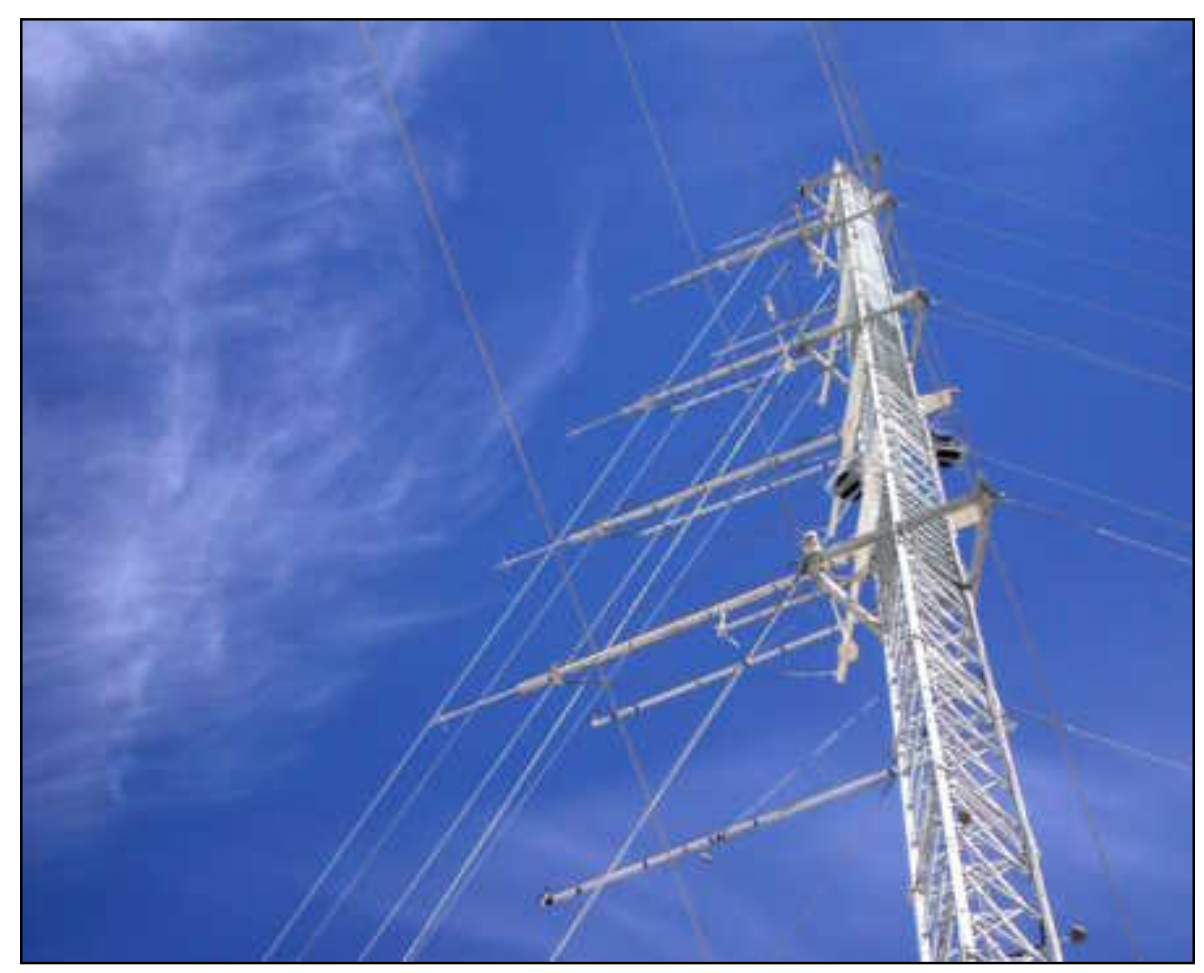

Figure 4-2. Close-up of NREL turbulence measuring instrumentation 


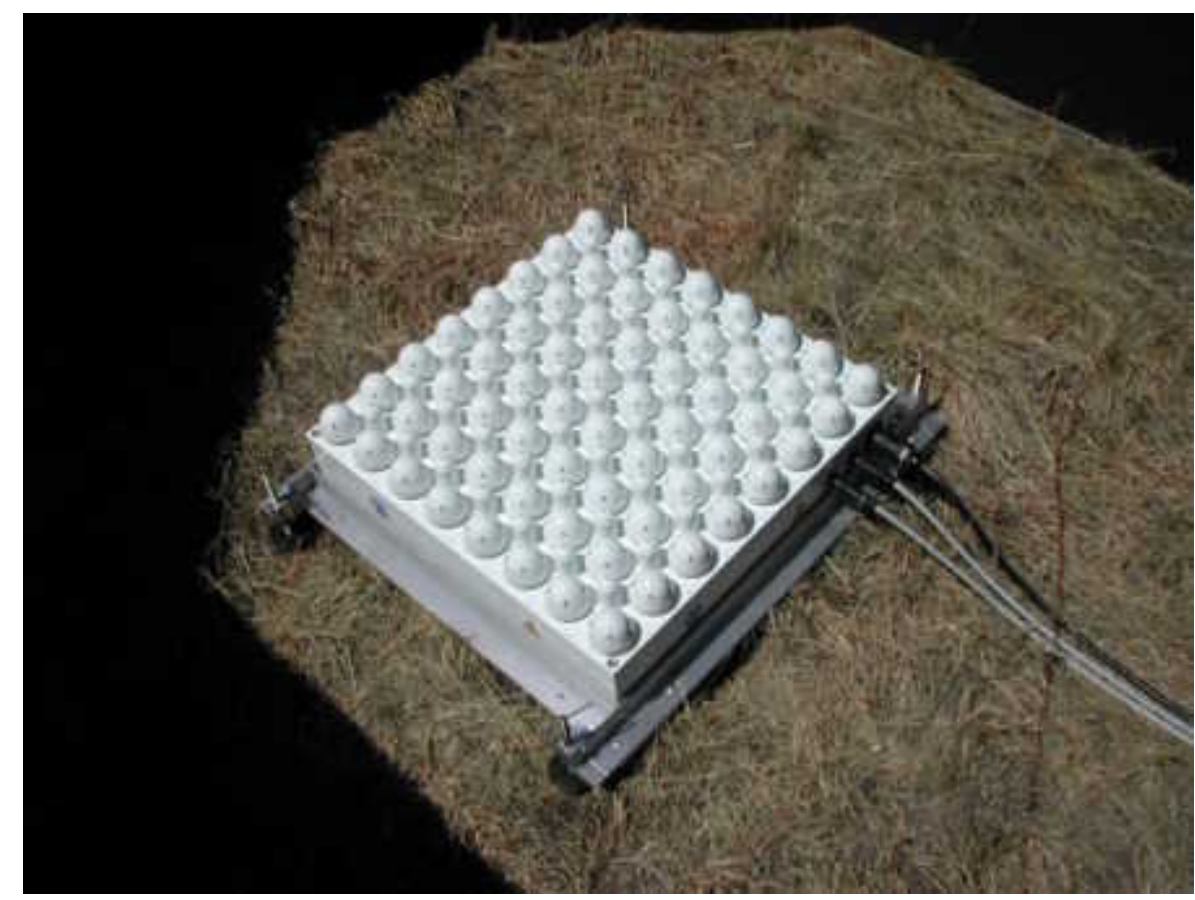

Figure 4-3. Scintec Model MFAS SODAR antenna 

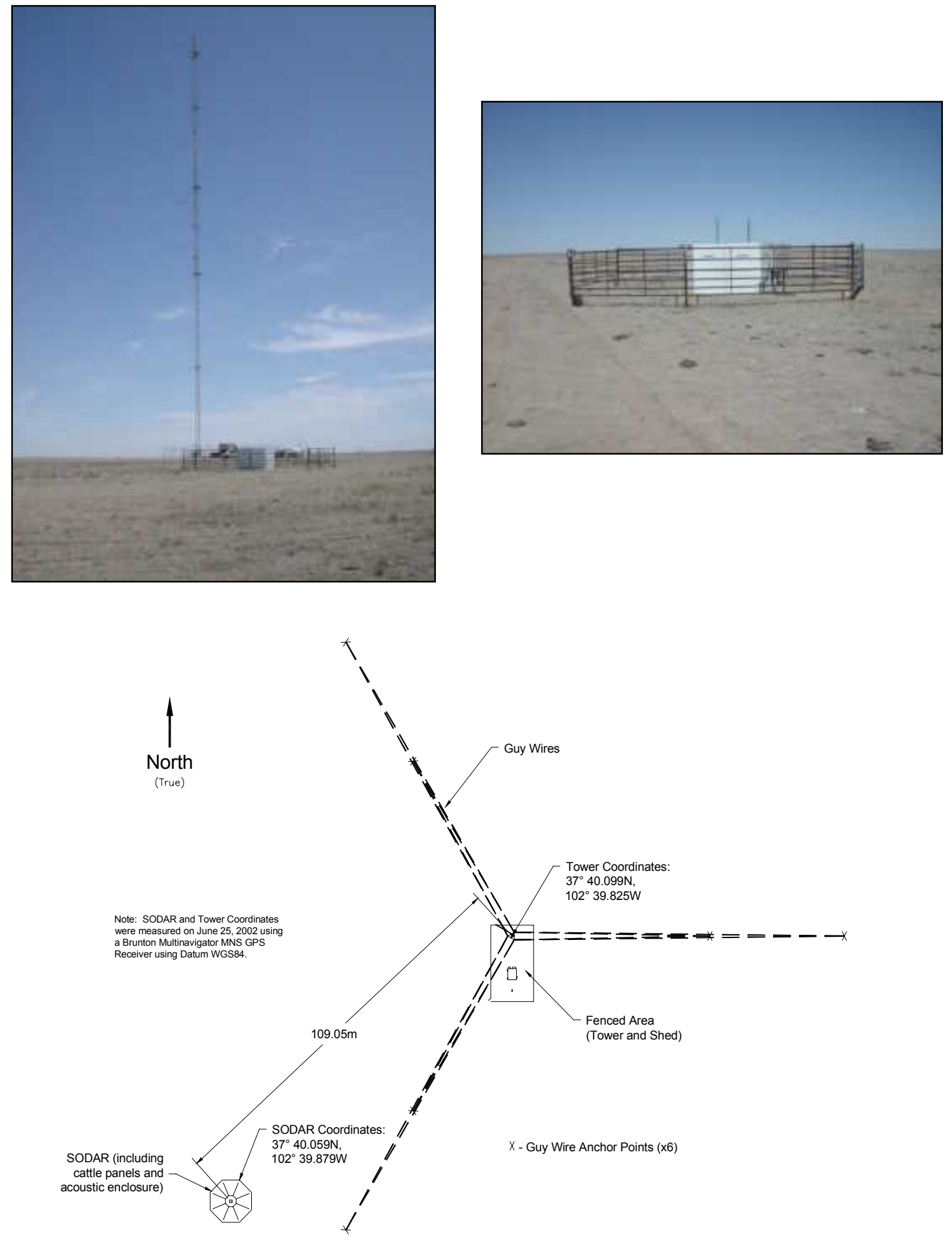

Figure 4-4. Installation and plan view of Scintec SODAR near GE Wind 120-m tower 

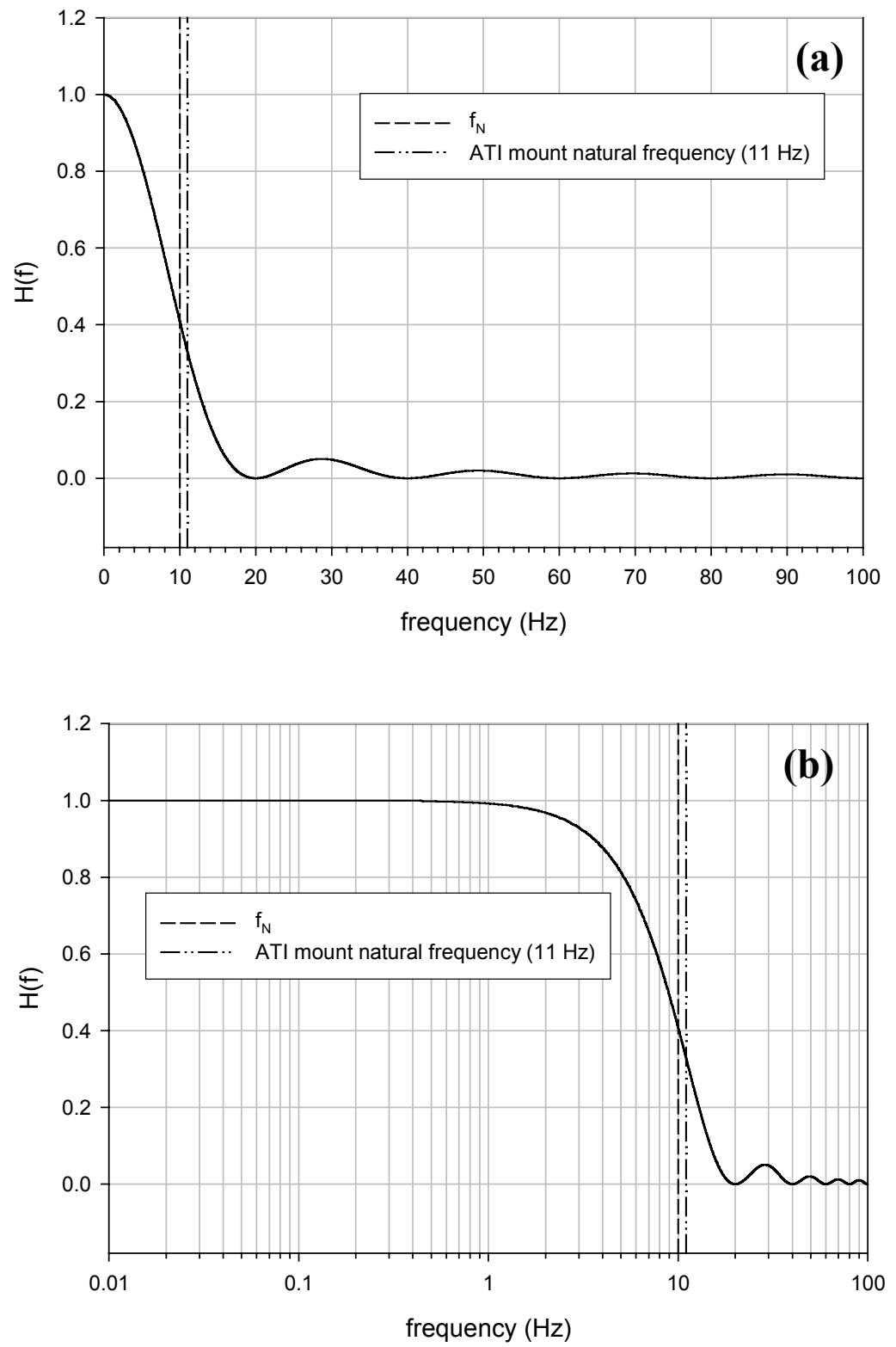

Figure 4-5. ATI sonic block-averaging filter frequency transfer function (200:20 frequency reduction) plotted in (a) linear and (b) semi-log coordinates 


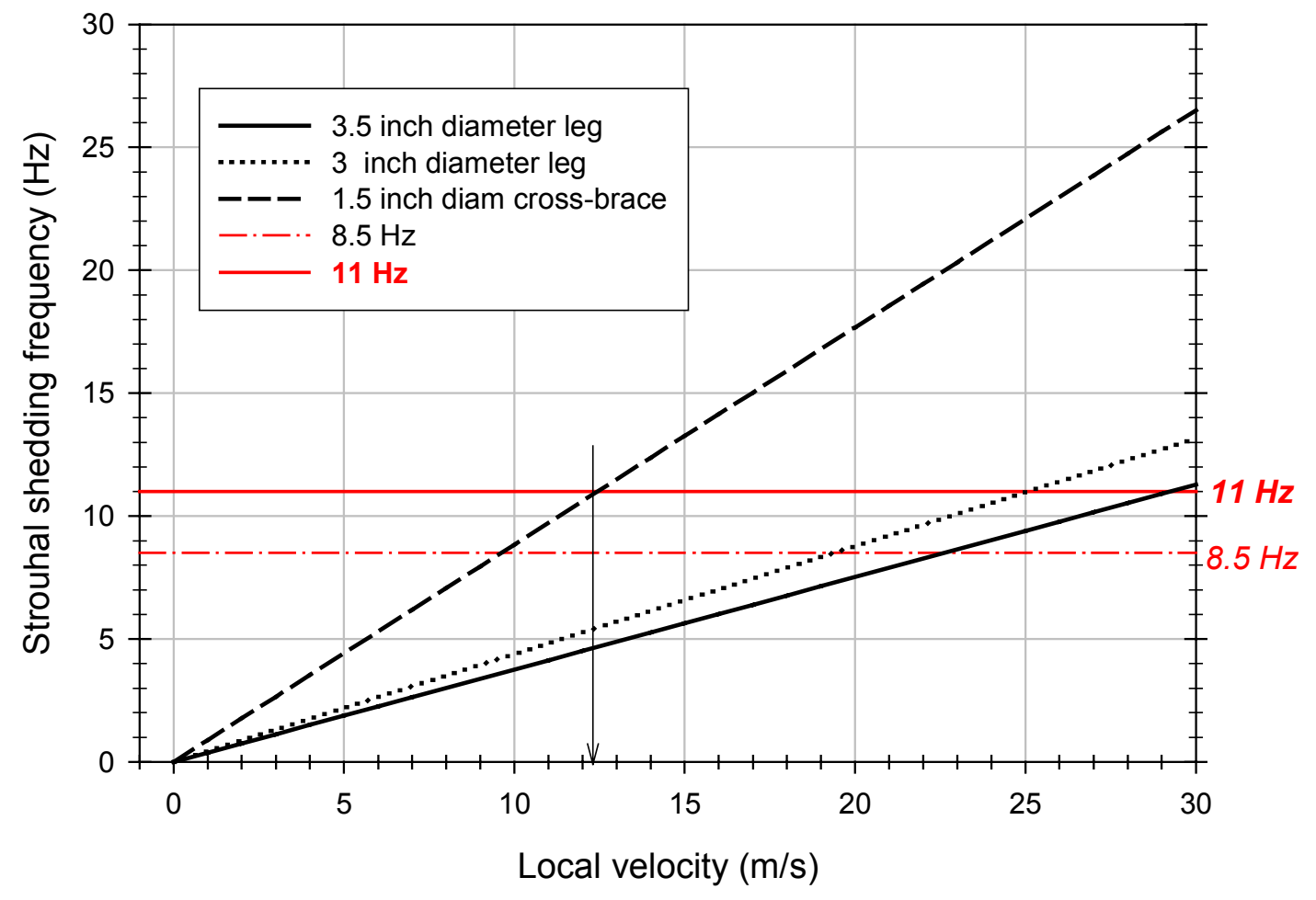

Figure 4-6. Aeolian vortex shedding frequencies as a function of wind speed from structural elements of GE Wind $120-\mathrm{m}$ tower 

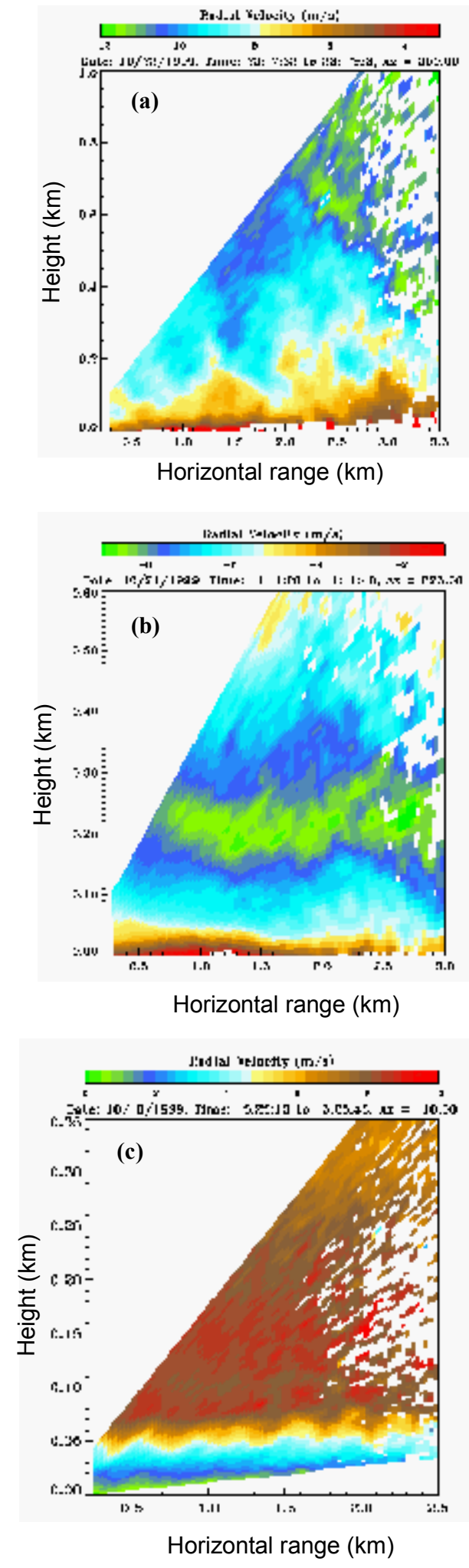

Example of NOAA LIDAR 30-Sec:

Observation of Day-Night Transition 17:07 CST - October 22, 1999

Observation of Weak

Low-Level Jet Formation 19:26 CST - October 21, 1999
Observation of Low-Level Jet With Shear-Gravity Waves 23:25 CST - October 6, 1999

Figure 4-7. Example of vertical wind structures as resolved by NOAA HighResolution Doppler LIDAR (HRDL) (Source: R. Banta, NOAA) 


\section{One-Year Statistical Summary}

In this section, we summarize the results of 1 year of data collection spanning the period October 5, 2001, through October 4, 2002. This data set allows us to document both the annual and seasonal variations of important operational parameters. For this summary, we have used the statistics based on the information continuously acquired every 5 minutes by the Campbell Scientific 23X data logger. We will discuss statistical summaries of monthly basic meteorological parameters and distributions of turbulence and gusts, vertical shear, and the dynamic stability of the 52- to 113-m layer that will eventually power the rotors of the GE Wind $1.5 \mathrm{~S}$ turbines installed at the LLLJP Site.

\subsection{Basic Meteorological Background Parameters}

\subsubsection{Atmospheric Thermodynamics}

The monthly mean air temperatures for the 3-, 52-, and 113-m heights, the mean temperatures for the 3- to -52-m and the 52- to 113-m layers, and the mean relative humidity for the 3-, 52-, and 113-m elevations are plotted in Figure 5-1. Clearly, during the colder months a distinct lowlevel temperature inversion (coldest at 3-m height and warmer above) was present, indicating strong static stability exists near the surface. During June through August, the mean 3-m air temperature was slightly warmer than those at the 52- and 113-m heights, signifying that the lower boundary layer was statically unstable in the mean. From April through August, the lower and upper (rotor) mean layer temperatures were almost identical, indicating very well mixed vertical conditions. The monthly vertical variation in relative humidity (Figure 5-1c) is indicative of the presence of vertical mixing during the warmer months when the differences were small. The large differences between the 3-m and upper heights during the colder periods were a result of the colder, statically stable air near the surface and the likely existence of an occasional evaporating snow cover.

Monthly mean values for air density (corrected for moisture) for the 3-, 52-, and 113-m levels and the 3- to 52-m and 52- to 113-m (rotor) layers are shown in Figure 5-2a,b. The International Civil Aviation Organization (ICAO) Standard Atmosphere temperatures for the 3-and 83-m heights (near hub elevation) are provided for comparison. The largest departures from standard values occurred during the warm-period (discussed in Section 2) months of April through September. The monthly variation in the mean surface $(3 \mathrm{~m})$ barometric pressure is plotted in Figure 5-2c. Again, the Standard Atmosphere value is provided for comparison, which indicates that, at least for this year, the surface pressure was higher than standard except for the month of August.

\subsubsection{Wind Energy, Shear, and Turbulence}

The monthly mean values of the energy density normalized by the percentage of the annual (24/7) mean are plotted in Figure 5-3a. This graph shows that the months of April, May, and June were the most energetic, with the months of October through January being the least energetic. This is generally consistent with the strong circulations present in the Great Plains during the spring months, though often March also had this characteristic. The increase in mean kinetic energy with height is demonstrated in Figure 5-3b. Typically, there was 3-4 times more energy available at the 52-m level (bottom of turbine rotor) than at the surface $(3 \mathrm{~m})$, and 4-8 
times as much at the 113-m height, with the largest differences occurring in the colder months. The increase seen between the 52- and 113-m heights was on the order of 1.5, with only a minor annual variation.

The variation in layer normalized monthly mean kinetic energy and accompanying mean vertical shear, as expressed by the shear exponent with mean wind direction, is shown in Figure 5-4. The 1/7 shear exponent is provided for reference. During the most energetic months (April through June), the mean wind direction was from the south-southeast with the mean shear exponent just slightly less than $1 / 7$. The colder surface temperatures and stable lower layer were occurring from October through March, with the higher shear values shown for the 3- to 52-m layer. During the summer months, when LLJ activity was more prevalent, the 52- to 113-m layer had a slightly larger mean shear value.

The dynamic stability of the rotor disk layer (52-113 $\mathrm{m}$ ) as expressed by the gradient Richardson number $(R i)$ exerts a significant influence on the inflow turbulence characteristics that turbines will operate in. This is well documented in Figure 3-3, where we noted that high root flapwise equivalent loads frequently occurred in weakly stable flows when the turbine layer $R i<+0.10$. At the LLLJP Site, because of the much higher hub height of the future GE 1.5S turbines $(80 \mathrm{~m})$, the layer occupied by the rotor disks ( 45 to $115 \mathrm{~m}$ ) is more appropriate. Our instrumentation provided a lowest elevation of $52 \mathrm{~m}$ (cup anemometer) or $54 \mathrm{~m}$ (sonic anemometer) and highest elevations of $113 \mathrm{~m}$ (cup anemometer) and $116 \mathrm{~m}$ (sonic anemometer). Thus, we will refer to the height range of 52-113 m or 54-116 m as the "rotor layer" depending on the source of the measurements involved (i.e., cup or sonic anemometers).

The relationship between the distribution of rotor layer mean wind speeds and the corresponding dynamic stability is, therefore, very important. A high frequency of turbine operations in nearto-above-rated wind conditions, when the dynamic stability of the rotor layer is in the weakly stable regime, will likely expose the turbines to increased coherent turbulence leading to higher loads and increased fatigue damage.

To assess this situation at the LLLJP Site, we have plotted the joint probability distribution of the rotor layer mean wind speed and the corresponding dynamic stability $(R i)$ in Figure 5-5 in which the critical stability range $\left(R i_{c}\right)$ has been indicated with a light cross hatch. While the most frequent combination of mean wind speed and stability occurs at about $11.5 \mathrm{~m} / \mathrm{s}$ and $R i=+0.19$, respectively, the wide range of mean wind speeds that have a significant probability within the critically stable range will result in significant operating time under these conditions. Perhaps more important, however, is the ridge-like peaks of higher joint probabilities that extend from near the rated wind speed up to a peak of more than $22 \mathrm{~m} / \mathrm{s}$ and just to the right of the vertical $R i$ $=0$ (neutral) scale line. This ridge line resides in a region where the $R i<+0.10$ (weakly stable) and indicates that a higher frequency of operations under such conditions can be expected. For example, in the extreme, the joint probability (according to Figure 5-5) of having a layer mean wind speed of $22 \mathrm{~m} / \mathrm{s}$ and a $R i=+0.06$ is about 0.005 or $0.5 \%$, but for a combination of $13.6 \mathrm{~m} / \mathrm{s}$ and $\mathrm{Ri}=+0.05$, the probably is about $0.5(5 \%)$ or an order of magnitude greater. Which combination contributes more fatigue damage from the increased frequency of encountering coherent turbulence depends on the relative frequency of occurrence of each of these conditions. 


\subsection{Turbulence Distributions}

\subsubsection{Turbulent Intensity Distributions}

The joint probability density distributions of turbulence intensity and mean wind speed for the 52- and 113-m heights (rotor layer) are presented in Figure 5-6. The distributions in these plots do not include data when the wind direction was such that the flow was coming through or near enough to the tower to cause a significant local flow distortion at the anemometer (i.e., wind directions $120 \pm 15^{\circ}$ ). The plots show that the characteristic wind speed at the $52-\mathrm{m}$ level is about $9.5 \mathrm{~m} / \mathrm{s}$, while it is $12 \mathrm{~m} / \mathrm{s}$ at the $113-\mathrm{m}$ level (the rated wind speed for the GE Wind $1.5 \mathrm{~S}$ turbine).

The joint probability distributions of turbulence intensity and the 52- to 113-m (rotor) layer gradient Richardson number (dynamic stability) are plotted in Figure 5-7. The critical Richardson number region $\left(R i_{c}\right)$ is also indicated by the cross hatched regions. Figure 5-7a shows that the highest turbulence intensities at the $52-\mathrm{m}$ level were associated with stability conditions in the upper end of the critical range (i.e., in the neighborhood of $R i=+0.2$ ). The same is true for intensities measured at the $113-\mathrm{m}$ height, but the intensity with the highest frequency is quite low (around 2\%). The distributions of Figure 5-7 show that while the intensities tend to be a bit higher at the 52-m level, there is a higher intensity tail at the $113-\mathrm{m}$ height (i.e., the intensity associated with the 0.02 density is about $18 \%$ at $52 \mathrm{~m}$, but $22 \%$ at 113 $\mathrm{m}$, indicating that occasional bursts of turbulence at these intensities occur with the largest values at the highest elevation).

\subsubsection{Turbulence Level (Wind Speed Standard Deviation) Distributions}

The joint distributions of the 52- and 113-m level wind speed standard deviations $\sigma_{U_{H}}$ or turbulence level as a function of the 52- to113-m (rotor) layer Richardson number are shown in Figure 5-8. Again, the critical $R i$ range $\left(R i_{c}\right.$ ) has been indicated. The highest probabilities again fall within the $R i_{c}$ range. The standard deviations associated with the highest probabilities tend to be associated with the larger values of $R i_{c}$. While of low-probability, the highest values of $\sigma_{U_{H}}(>2.0 \mathrm{~m} / \mathrm{s})$ are found near $R i=0$.

In Figure 5-9, we present the observed frequency distributions of $\sigma_{U_{H}}$ derived from cup anemometer measurements at the 3-, 52-, and 113-m heights as a function of the mean wind speed at those heights. We have also provided curves plotting the variation of $\sigma_{U_{H}}$ as defined by the IEC Standard [13]. While the observed distribution of $\sigma_{U_{H}}$ at the 3-m level (Figure 5-8c) did reach at least the IEC "B" turbulence level reasonably frequently, $\sigma_{U_{H}}$ at the 52 - and $113-\mathrm{m}$ levels reached either the IEC "A" or "B" levels only infrequently (i.e., less than 0.2 hours over the entire year). Thus, the background turbulence level at the LLLJP site rarely exceeded the IEC specifications. This can also be seen in the presentation of Figure 5-10 in which we plotted the characteristic variations in $\sigma_{U_{H}}$ for each height as a function of the mean wind speed at that height. Again, the background characteristic turbulence levels were much lower than the IEC design level. This would suggest, based on that criteria, that turbulence-induced loads should not be a major issue in this inflow environment. 


\subsubsection{Gust Distributions}

The probability distributions for peak gusts, as derived from the cup anemometers installed at the 3-, 52-, and 113-m levels, are plotted in Figure 5-11. An estimate of the distribution for the 80-m height (hub of GE Wind 1.5S turbine) has also been provided.

\subsection{Vertical Shear Distribution}

The statistics regarding the vertical shear of the wind speed can be calculated for two layers with the availability of cup anemometers at heights of 3,52 , and $113 \mathrm{~m}$. The magnitudes of the shears are expressed in terms of the shear exponent $\alpha$, which is defined by the power law wind speed profile

$$
U_{2}=U_{1}\left(z_{2} / z_{1}\right)^{\alpha}
$$

as

$$
\alpha=\ln \left(U_{2} / U_{1}\right) / \ln \left(z_{2} / z_{1}\right)
$$

where $U_{2}$ and $U_{1}$ are the mean wind speeds at the corresponding heights $z_{2}$ and $z_{1}$. The IEC Standard [13] defines the Normal Wind Profile (NWP) as having a shear exponent of 0.2. We have included this on many of the plots that follow. Frequency distributions of the shear exponents for the 3- to 52-m and 52- to 113-m layers have been calculated for each month from October 2001 through September 2002 and for the entire annual period. The 52- to 113-m layer closely corresponds to the layer in which the GE $1.5 \mathrm{~S}$ turbine rotors will reside (the actual height range will be 45 to $115 \mathrm{~m}$ ). Figure 5-12 plots the annual observed frequency distributions for the two layers when the mean wind speed at $52-\mathrm{m}$ was more than $3 \mathrm{~m} / \mathrm{s}$. It is clear that the shear distribution in the upper (rotor) layer is broader with and more noticeably skewed toward high positive shears. The dominant distribution peaks for the lower and upper layers occurred at 0.102 and 0.069 , respectively. Figures 5-13 and 5-14 display the frequency distributions of the 3- to 52-m and 52- to 113-m (rotor) layers, respectively, for the cold season (October - March). The warm season (April - September) distributions are similarly presented in Figures 5-15 and 5-16. The 3- to 52-m distributions have a tendency to be multimodal, whereas the 52- to $113-\mathrm{m}$ distributions are generally unimodal, with except perhaps during July through September when there is a slight secondary mode seen between shear exponents of 0.2 and 0.4 .

We found that the observed annual shear exponent frequency distributions could each be approximated by two Gaussian distributions with the point of overlap at $\alpha=1 / 7$, as shown in Figure 5-17. The probability density of the Gaussian distribution is given by

$$
p_{\alpha}=B \exp \left(-0.5((\alpha-C) / D)^{2}\right)
$$

where $B, C, D$ are constants that depend on the layer (3-52 $\mathrm{m}$ or $52-113 \mathrm{~m}$ ) and if $\alpha$ is $\leq 1 / 7$ or $\alpha$ $>1 / 7$. Table 5-1 lists the appropriate values for the constants.

By plotting the observed shear exponent distributions for the 52- to 113-m layer above and below the $\alpha=1 / 7$ overlap point against the Richardson number for that layer, we found shear exponents that are less than $1 / 7$ are only slightly correlated with $R i$. However, for exponents larger than $1 / 7$, they are frequently associated with $R i$ values within the critical range. In the 3to $52-\mathrm{m}$ layer, exponents greater than 1/7 that correspond to a distribution of $R i$ values, are very 
peaked within the critical range. For values less than $1 / 7$, however, the $R i$ values exhibit a more moderate peak within the weakly stable region of values less than +0.05 . These results are plotted in Figure 5-18.

We found that in the 52- to 113-m (rotor) layer, high mean vertical shears are less likely. This result is demonstrated by the data plotted in Figure 5-19. The graph shows the number of cumulative hours by month, season, and annually, when the 52- to 113-m layer shear exponent exceeded 0.2 for a range of mean wind speed at $52 \mathrm{~m}$ (at the lowest rotor elevation). Because the variation with wind speed on this graph is dominated by speeds below $10 \mathrm{~m} / \mathrm{s}$, we plotted the annual variation of the shear exponent distributions again as a function of the mean wind speed at $52 \mathrm{~m}$ over a higher, but narrower range (Figure 5-20). These graphs demonstrate that the turbine rotors will be experiencing shears larger than the IEC NWP value (0.2) most frequently at mean wind speeds below and just above rated operation $(12 \mathrm{~m} / \mathrm{s})$.

\subsection{Vertical Dynamic Stability}

Whiteman et al. [16] found the existence of both northerly and southerly low-level jets. The southerly ones are more frequent with the greatest number occurring during the warm season and at night. Also important are the conditions that produce coherent turbulent motions beneath jet maximums. We have seen in Section 2 that highly sheared, stable layers that exhibit gradient Richardson numbers in the critical range $(0<R i<0.25)$ can develop $\mathrm{KH}$ instabilities that, in turn, can generate bursts of coherent turbulent energy. Thus, the frequency of conditions in which the flow within the rotor layer $(52-113 \mathrm{~m})$ is dynamically unstable (within the $R i_{c}$ ) and are associated with wind directions that favor LLJ development (such as southerly winds during the warm season) are important to turbine operations.

Figures 5-21 and 5-22 display the joint probabilities of the 52- to 113-m layer $R i$ and the mean wind direction at $52 \mathrm{~m}$ for the cold season and warm seasons, respectively. The frequency distribution of $R i$ is also provided. The highest joint frequency is shown in deep red with the least in dark blue. These diagrams indicate the range of the wind directions that are occurring with high frequencies of critically stable flows within the future rotor disk.

Figure 5-21 demonstrates that, during October 2001, shear-flow instabilities could have occurred with any wind direction, though the preferred range was from the southeast through the northwest $\left(135\right.$ to $\left.315^{\circ}\right)$. A somewhat similar pattern was observed in November. There is a definite shift toward the north in December, with the most favored wind direction being northerly in January and February, though critical flow conditions can again occur from any wind direction. In March, the pattern returned to one similar to that seen in October, with the frequent critical flows occurring with winds from the east $\left(90^{\circ}\right)$ through northwest, with a southsouthwesterly direction $\left(225^{\circ}\right)$ being the most prevalent. This sequence of events agrees well with Whiteman's comments regarding the observations of low-level jets (i.e., jets occurring with cold air outbreaks following frontal passages).

The warm season distributions displayed in Figure 5-22 present a much different picture. As the weather became warmer, the range of wind directions associated with critically stable flows became increasingly narrower and eventually centered near a southerly direction $\left(\sim 170^{\circ}\right)$. This narrowing of the range of wind directions was accompanied by a significant increase in the frequency of critically stable flows, as indicated by the $R i$ distributions. This is consistent with the high frequency of southerly jets found by Whiteman during this season. It also during these 
months that there is highest likelihood of turbine rotors encountering shear flow instabilities in the nocturnal boundary layer and, as a result, the greatest exposure to coherent turbulent activity.

The cumulative number of hours that a turbine would have encountered $R i_{c}$ flow instabilities in terms of a series of mean wind speed categories is shown by month, season, and annually in Figure 5-23. During the cold season, turbines operating in wind speeds in the range of $8-13 \mathrm{~m} / \mathrm{s}$ would have encountered dynamic instabilities in the neighborhood of 400 hours, increasing to about 700 hours during the warm season. Annually the number of hours operating in such conditions was about 1100 hours.

We have shown in Sections 1 and 3 that the largest loading events occur in highly sheared flows that are weakly stable (i.e., $R i<+0.10$ ). In Figure 5-24, we provide the observed distribution of the number of hours in which weakly stable and critically stable $(R i<+0.25)$ flow conditions were observed by month, season, and annually. Clearly, the months of April, May, and June would have likely presented the greatest operational challenge for turbine operations. Conditions known to create large loading events occurred more than 300 hours $(14 \%)$ of the time during those 3 months. The number of hours in which weakly stable conditions existed doubled during the warm season compared to the cold (i.e., from 200 hours during the cold months to 400 hours during the warm). Over the entire year, critically stable flows were seen for a total of 1800 hours $(21 \%)$ with the more challenging weakly stable conditions occurred a third of that time $(7 \%)$ annually.

\subsection{Conclusions}

We have reached the following conclusions from the analysis of the 1-year statistical data set collected from October 2001 through September 2002:

- The highest available energy density at the LLLJP Site occurred in late spring and early summer (April through June)

- The highest turbulence intensities occurred in the warm season (April through September) with the highest mean values in the 52- to 113-m (rotor) layer (10-12\%) seen in June and July

- The highest turbulence intensities were seen when the 52- to 113-m (rotor) layer gradient Richardson number was within the critical range $(0<R i<0.25)$

- For all practical purposes, the observed mean turbulence levels (wind speed standard deviations) observed at the 52-113-m levels remained below both the IEC "A" and "B" specifications

- The 0.1-percentile peak gust or extreme velocity within the turbine rotor disk was $30 \mathrm{~m} / \mathrm{s}$

- The distribution of vertical shear over the rotor layer as expressed by the shear exponent had a positive skew with a significant tail, which frequently exceeds the IEC NWP value of 0.2

- The annual shear exponent density distributions can be approximated by two Gaussian distributions: one for shear exponents $\leq 0.143(1 / 7)$ and the other for exponents $>0.143$

- Shear exponents $>1 / 7$ in the 52- to $113-\mathrm{m}$ (rotor) layer were highly correlated with critical range stability $\left(R i_{c}\right)$

- Extreme values of shear decreased as the mean wind speed increased 
- The highest values of shears seen by operational turbines occurred most often within a hubheight mean speed range of $8-14 \mathrm{~m} / \mathrm{s}$

- The warm season (April through September) was dominated by southerly winds in which LLJs were known to form frequently (mostly at night)

- Flow conditions that were potentially challenging for turbine operations could be expected to occur up to $16 \%$ of the time annually when the layer was critically stable, the shear exponent was in excess of 0.2 , and the hub mean wind speed was between 8 and $13 \mathrm{~m} / \mathrm{s}$. 
Table 5-1. Gaussian Shear Distribution Coefficients

\begin{tabular}{cccc}
\hline $\begin{array}{l}\text { 52- to 113-m } \\
\text { Layer }\end{array}$ & B & C & D \\
\hline$\alpha \leq 1 / 7$ & 1.1672 & 0.18542 & 0.25172 \\
$\alpha>1 / 7$ & 3.0838 & 0.05154 & 0.07064 \\
\hline 3- to 52-m Layer & & & \\
\hline$\alpha \leq 1 / 7$ & 2.7706 & 0.18217 & 0.11569 \\
$\alpha>1 / 7$ & 5.6118 & 0.09845 & 0.03912 \\
\hline
\end{tabular}




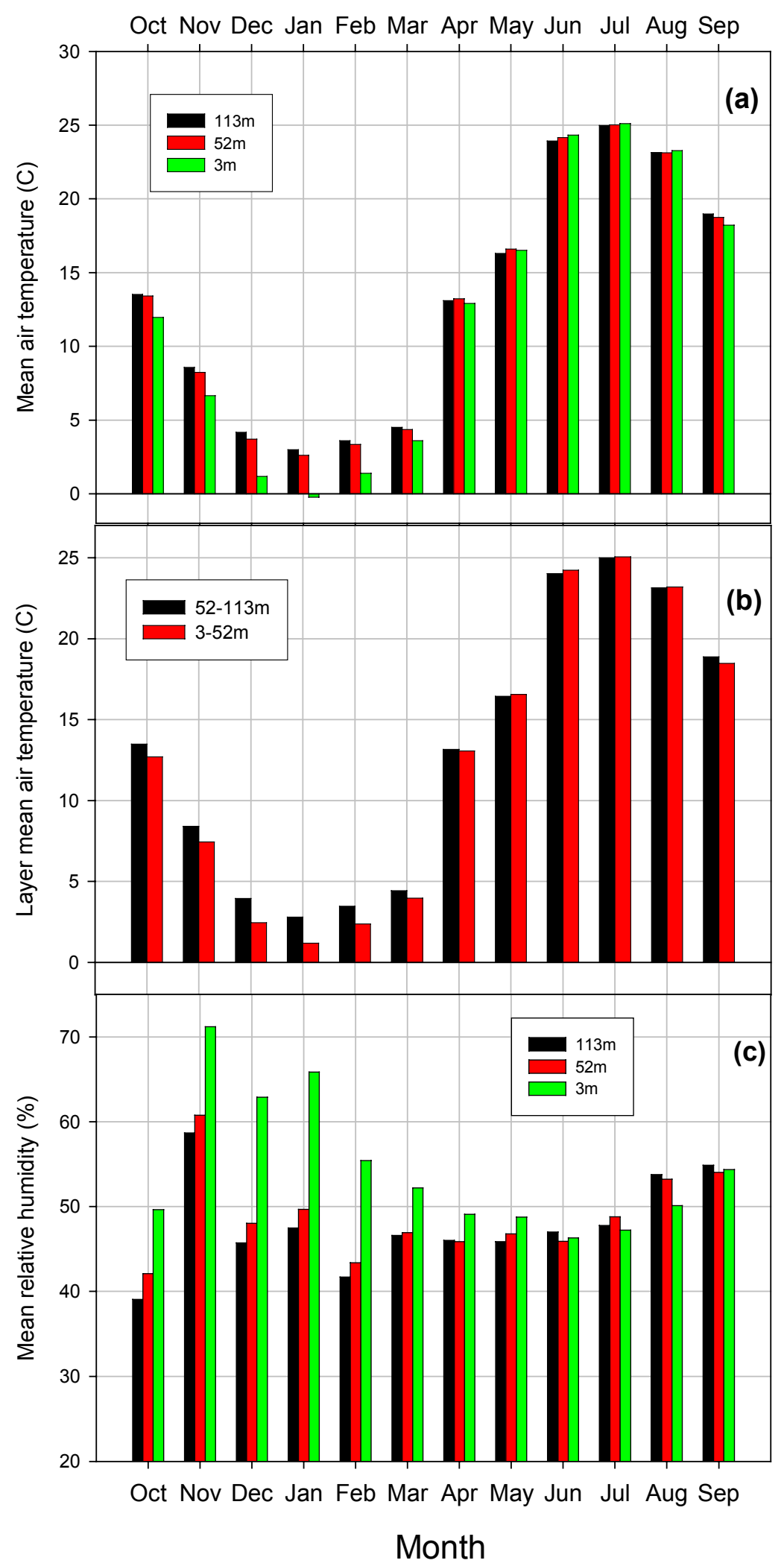

Figure 5-1. Annual variation of monthly mean temperatures, layer mean temperatures, and mean relative humidity from GE Wind $120-\mathrm{m}$ tower for the period of October 2002 through October 2003 


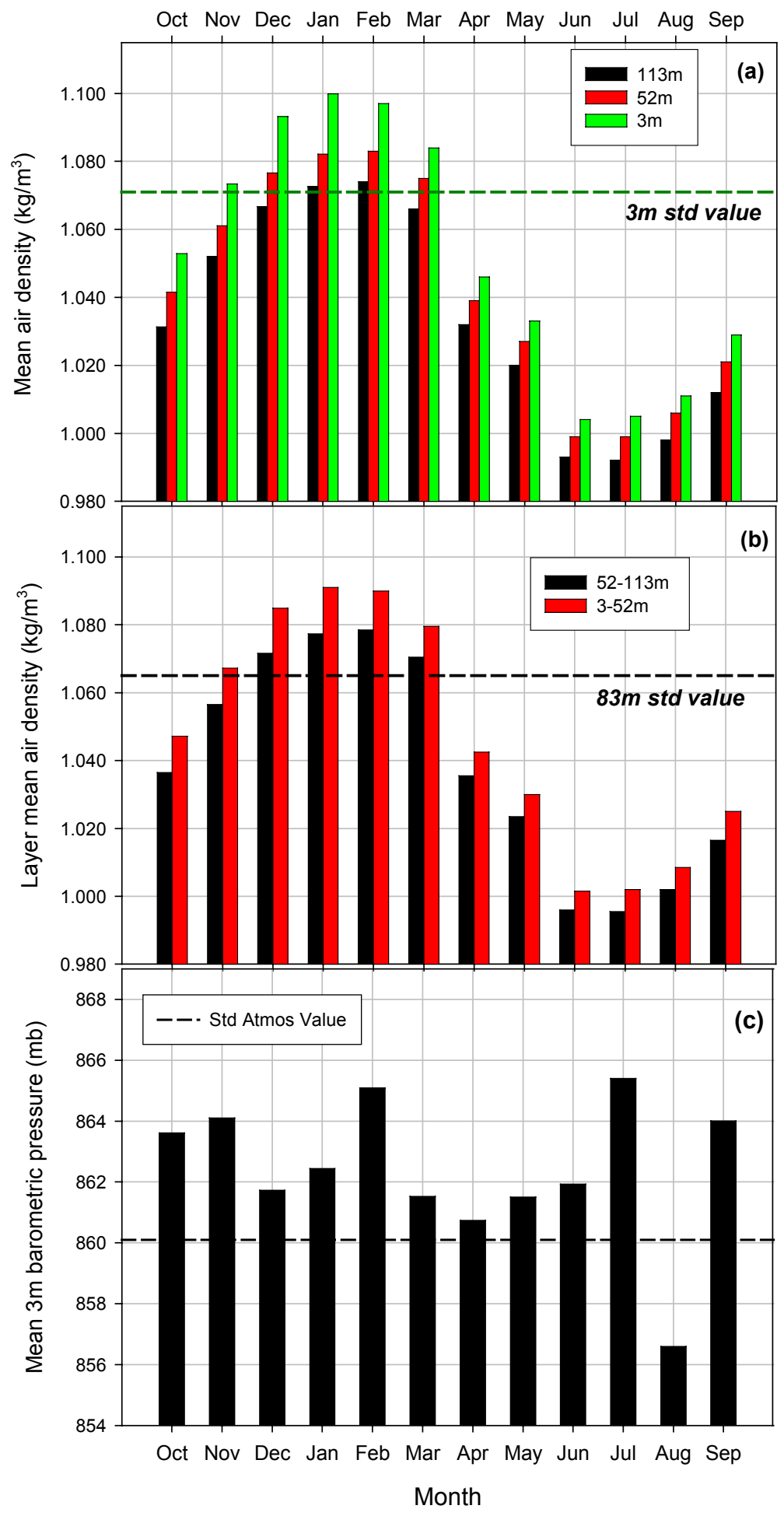

Figure 5-2. Annual variation of monthly mean air densities, layer mean air densities, and 3-m barometric pressure from GE Wind 120-m tower for period of October 2001 through October 2002: (a) air density, (b) layer mean air density, (c) mean barometric pressure 


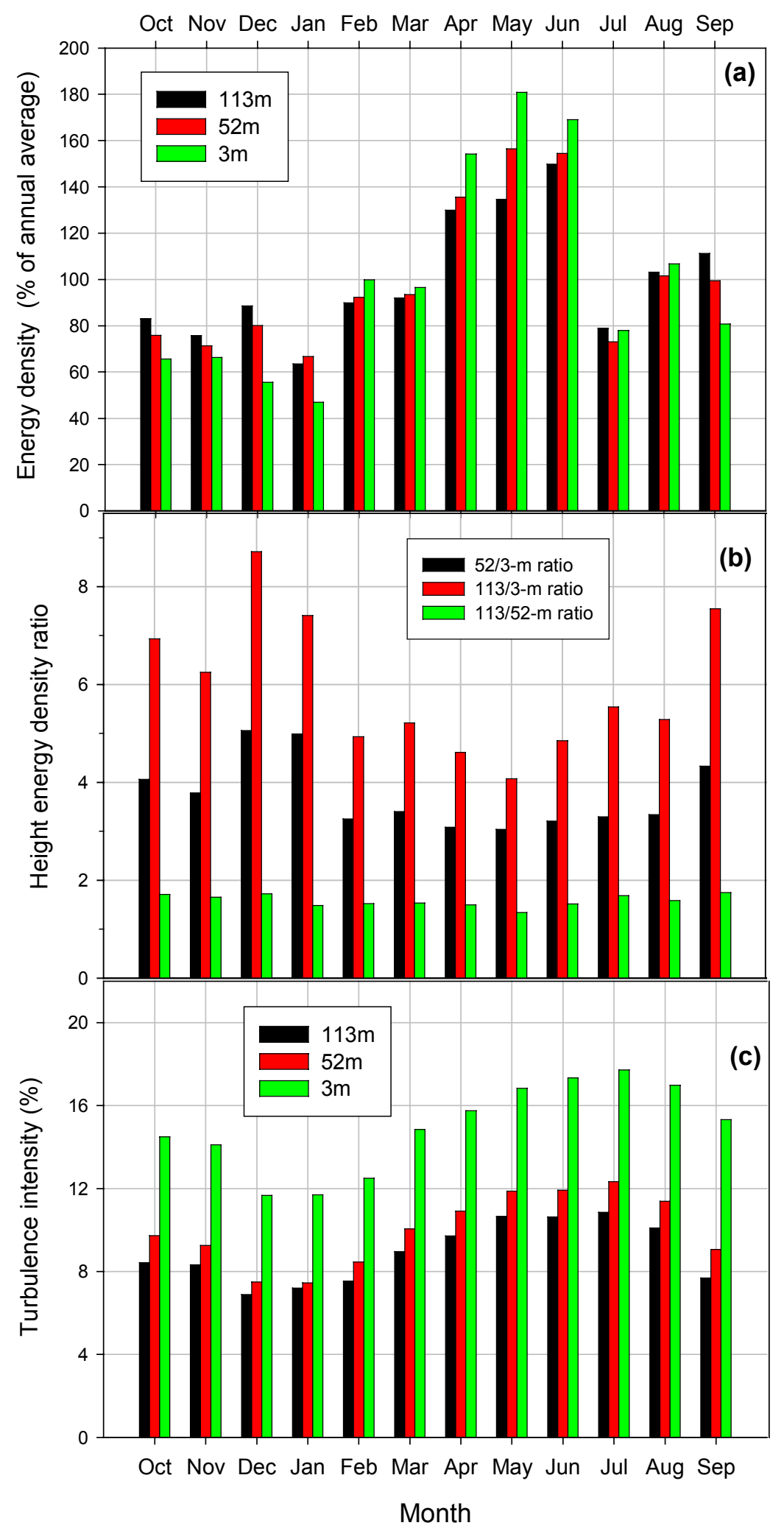

Figure 5-3. Annual variation of monthly normalized energy density, height energy density ratio, and turbulence intensity from GE Wind $120-\mathrm{m}$ tower for period of October 2001 through October 2002: (a) normalized energy density, (b) height/energy density ratio, (c) turbulence intensity 


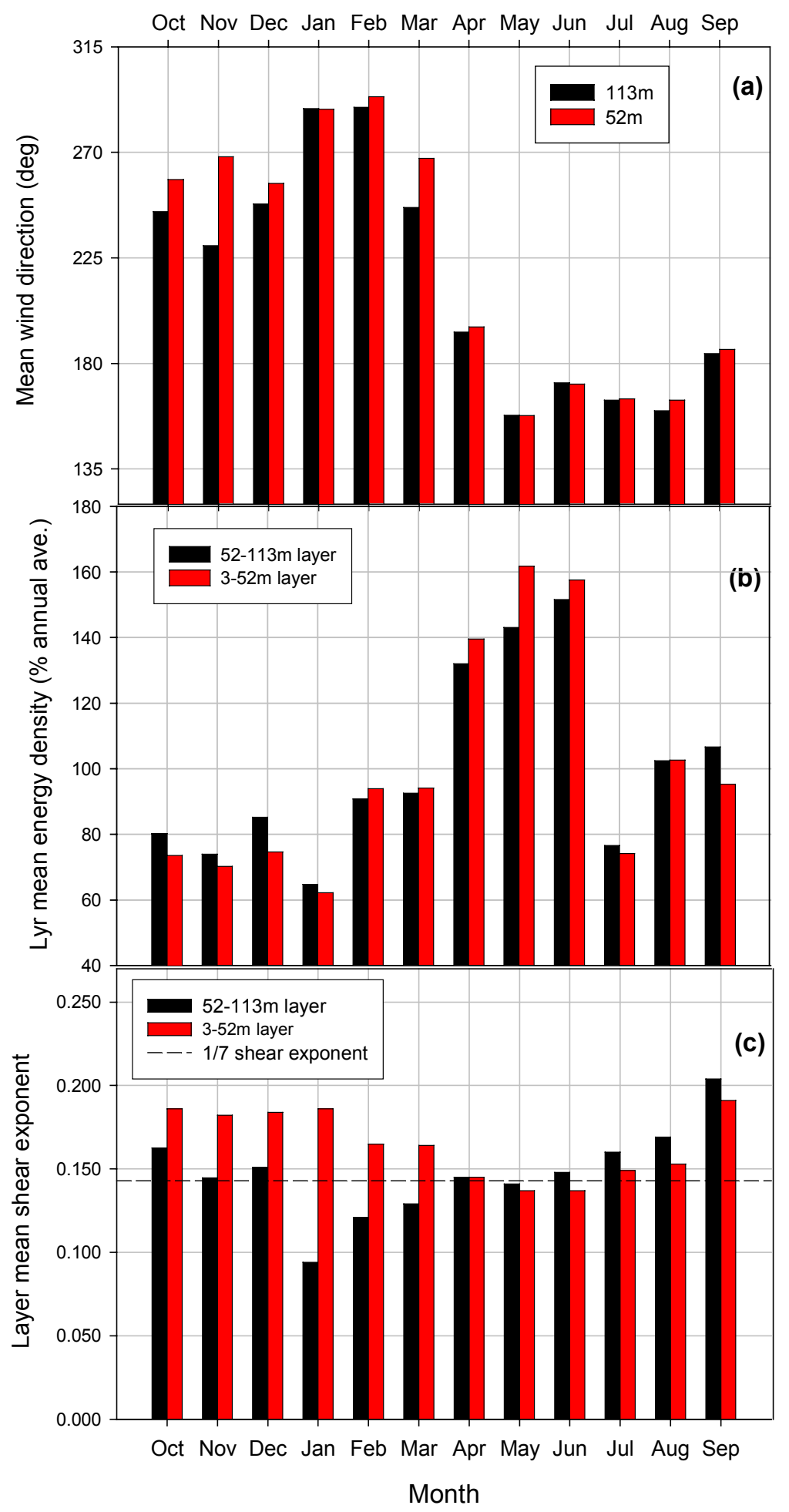

Figure 5-4. Annual variation of monthly mean wind directions, normalized layer mean energy densities, and layer mean shear exponents from GE Wind 120-m tower for period of October 2001 through October 2002: (a) wind direction (a) wind direction, (b) normalized energy density, (c) shear exponent 


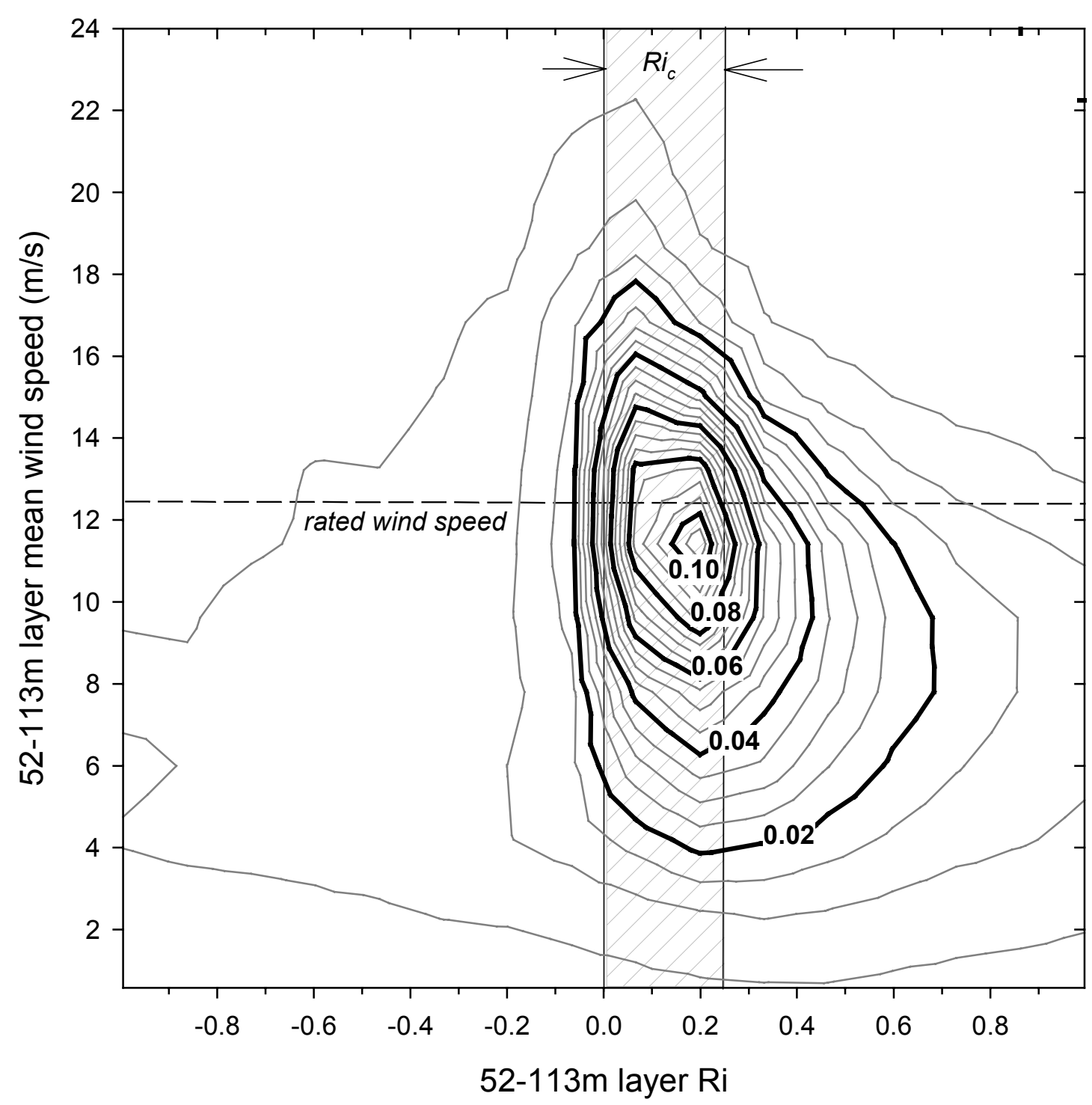

Figure 5-5. Annual joint probability distributions for the mean wind speed at the 52- to 113-m layer and the 52- to 113-m layer $R i$ 

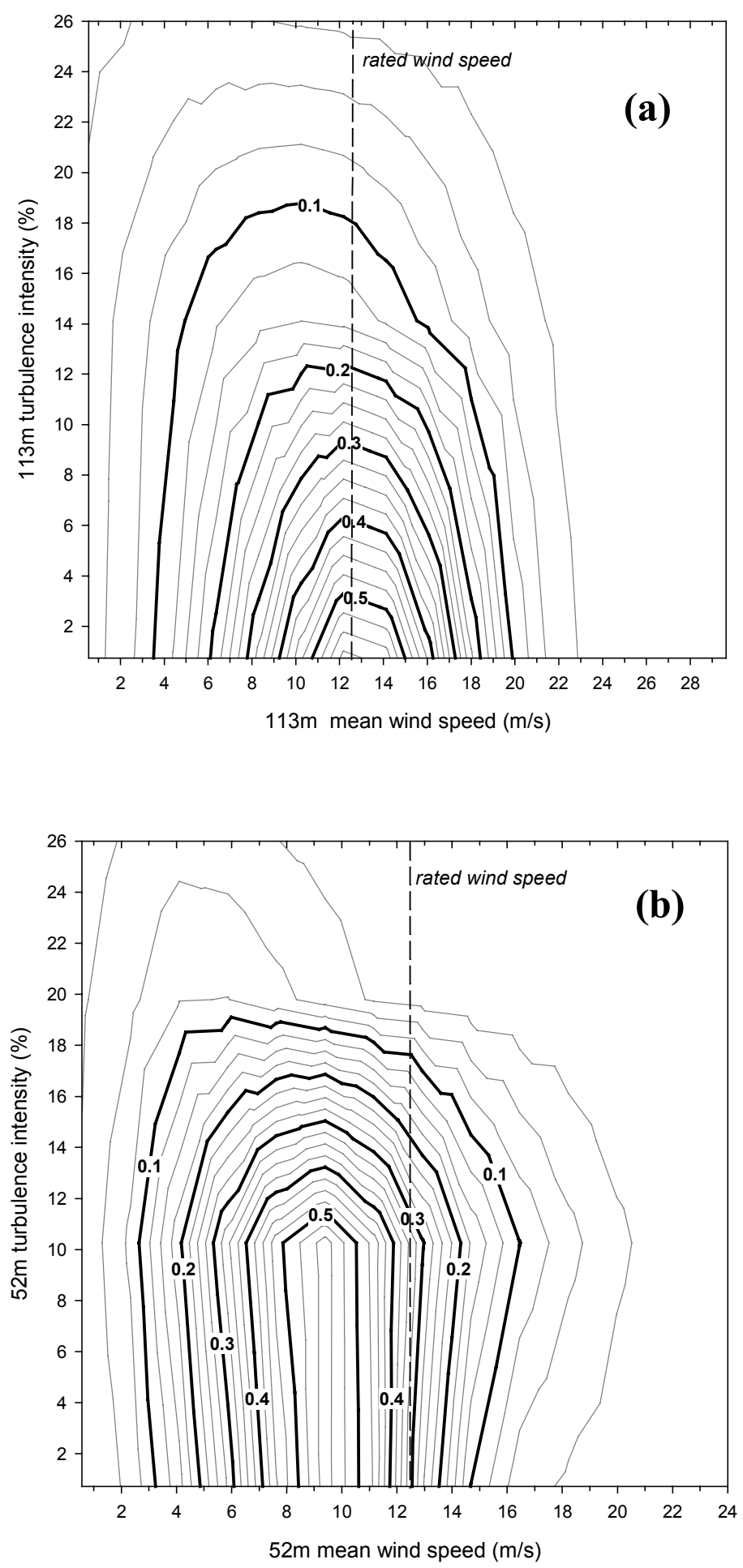

Figure 5-6. Annual joint probability distributions of turbulence intensities and mean wind speed: (a) $113 \mathrm{~m}$, (b) $52 \mathrm{~m}$ 

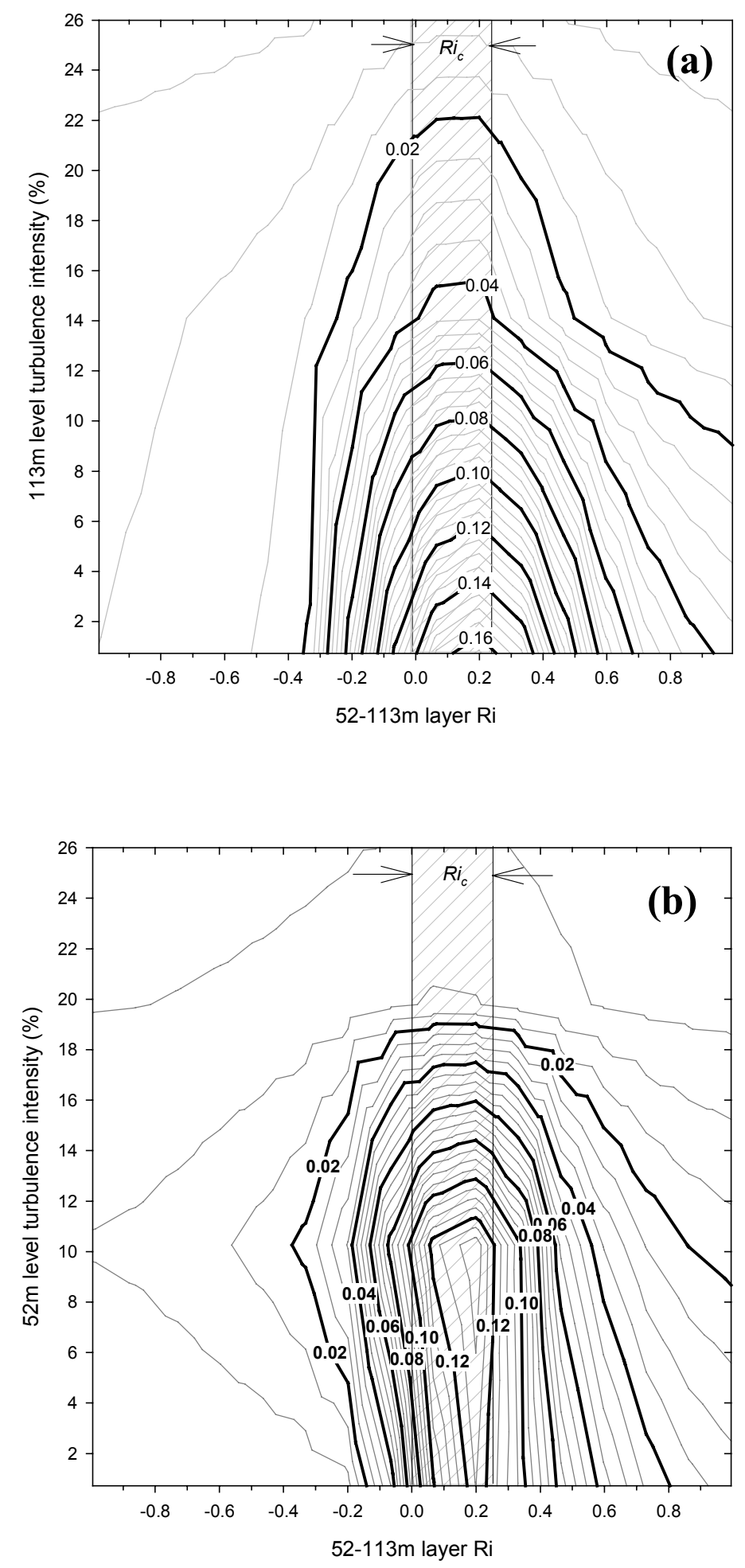

Figure 5-7. Annual joint probability distributions of turbulence intensities and the Richardson number Ri at the 52- to 113-m layer: (a) $113 \mathrm{~m}$, (b) $52 \mathrm{~m}$ 

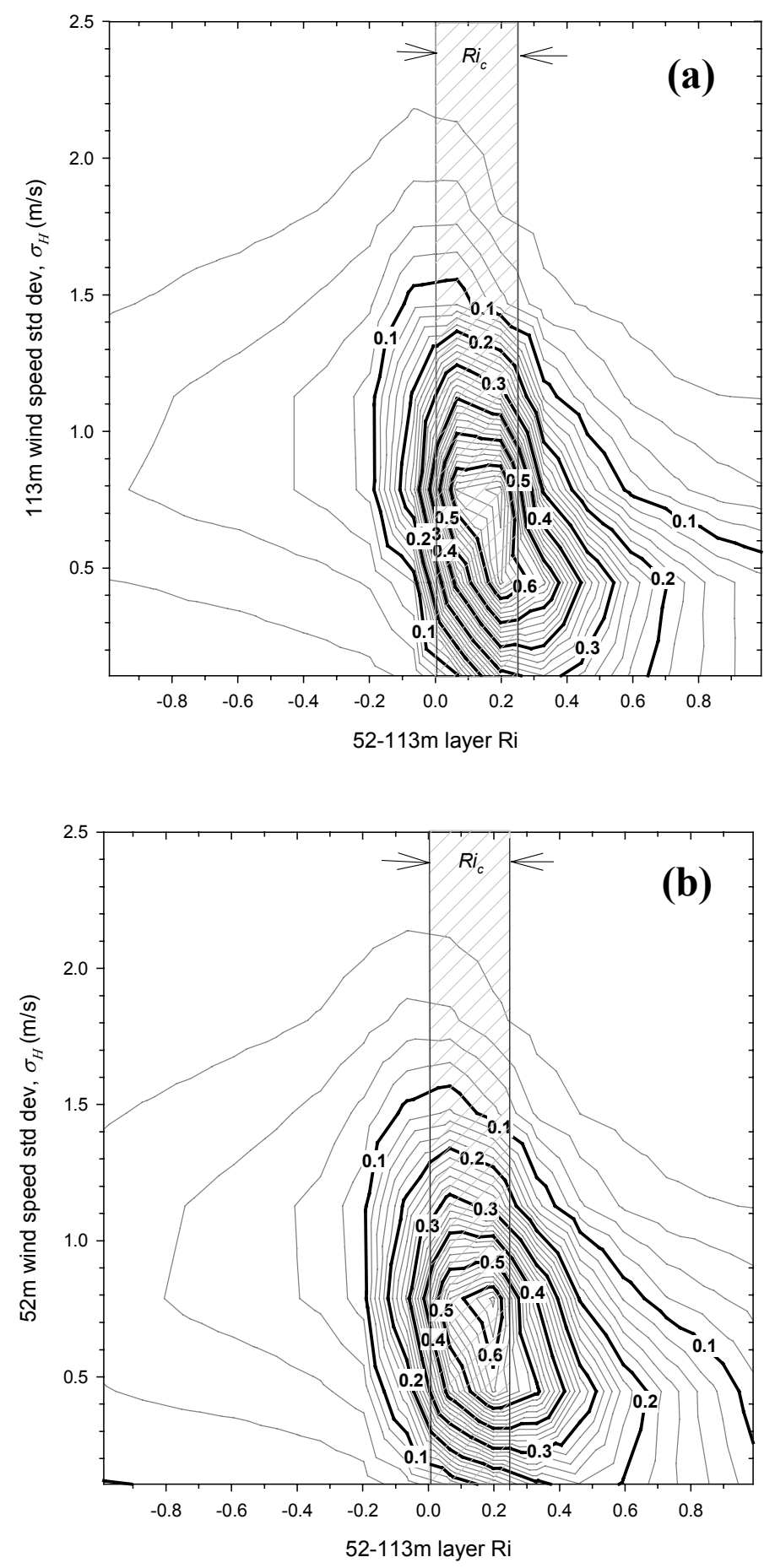

Figure 5-8. Annual joint probability distributions of wind speed standard deviation $\sigma_{H}$ and Richardson number $R i$ at the 52- to 113-m layer: (a) 113 $\mathrm{m}$, (b) $52 \mathrm{~m}$ 

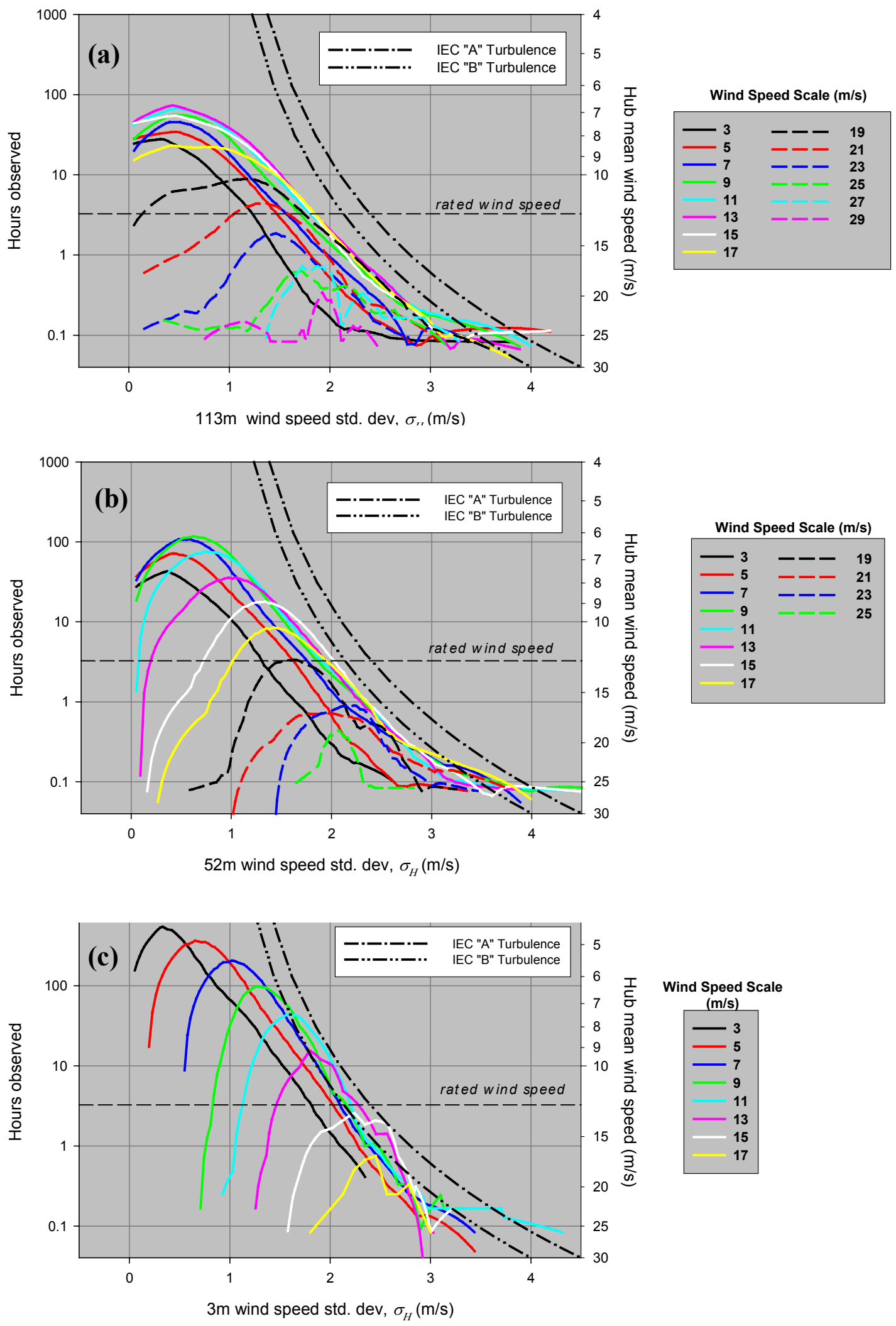

Figure 5-9. Annual probability distributions of wind speed standard deviations $\sigma_{H}$ at a height of (a) $113 \mathrm{~m}$, (b) $52 \mathrm{~m}$, and (c) $3 \mathrm{~m}$ 


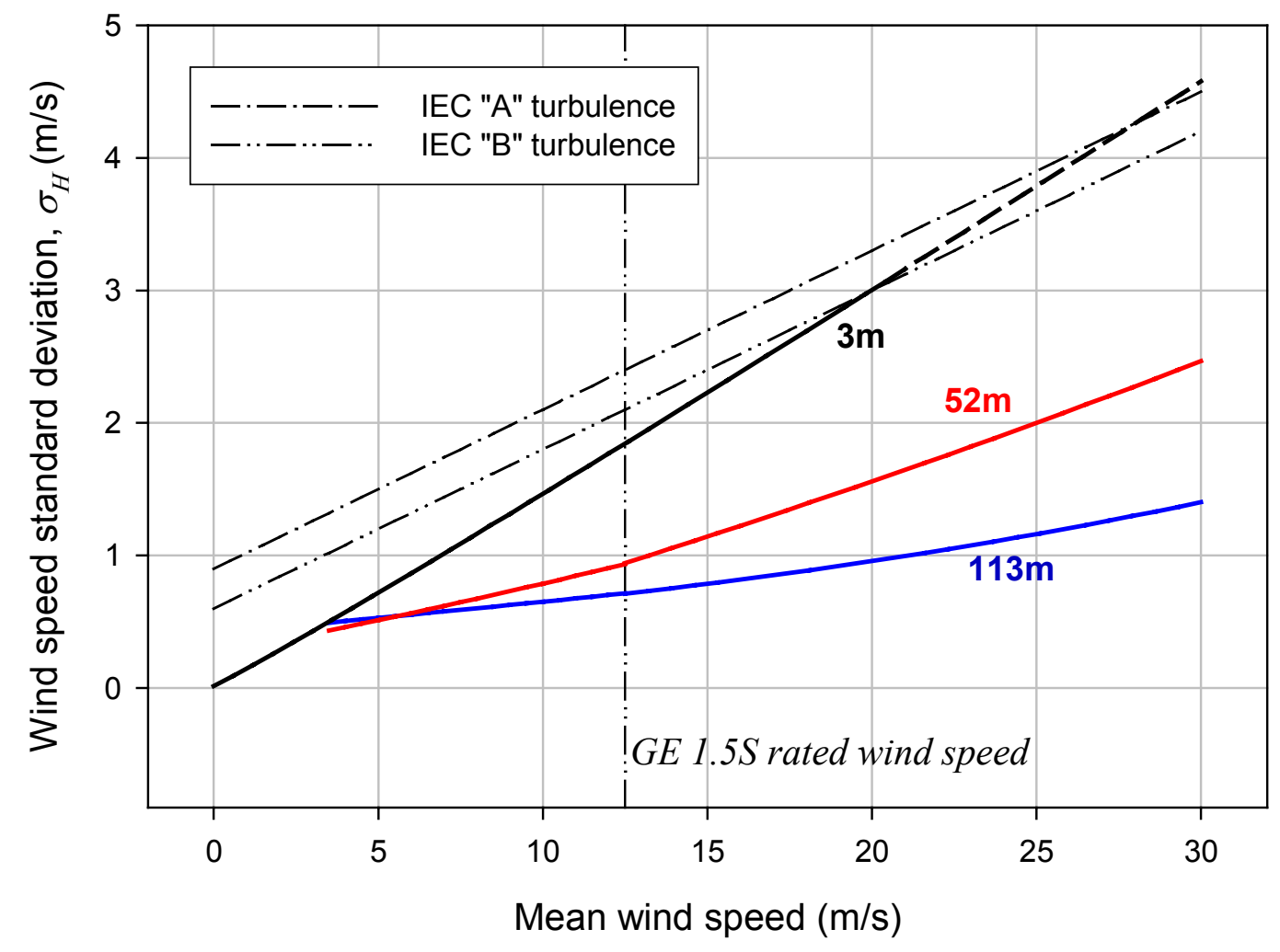

Figure 5-10. Population characteristic wind speed standard deviation $\sigma_{H}$ as a function of mean wind speed 


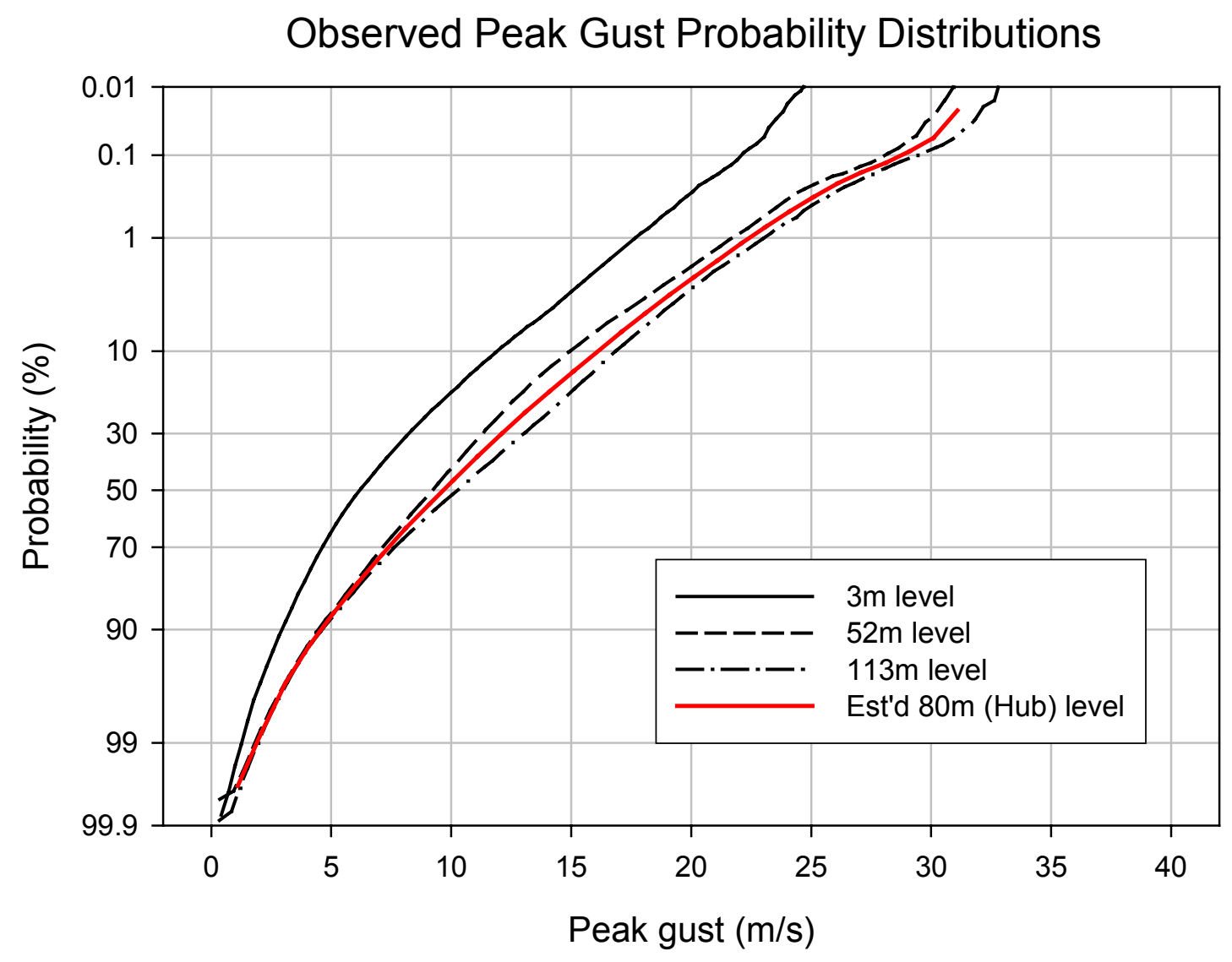

Figure 5-11. Annual probability distribution of peak gust velocities 


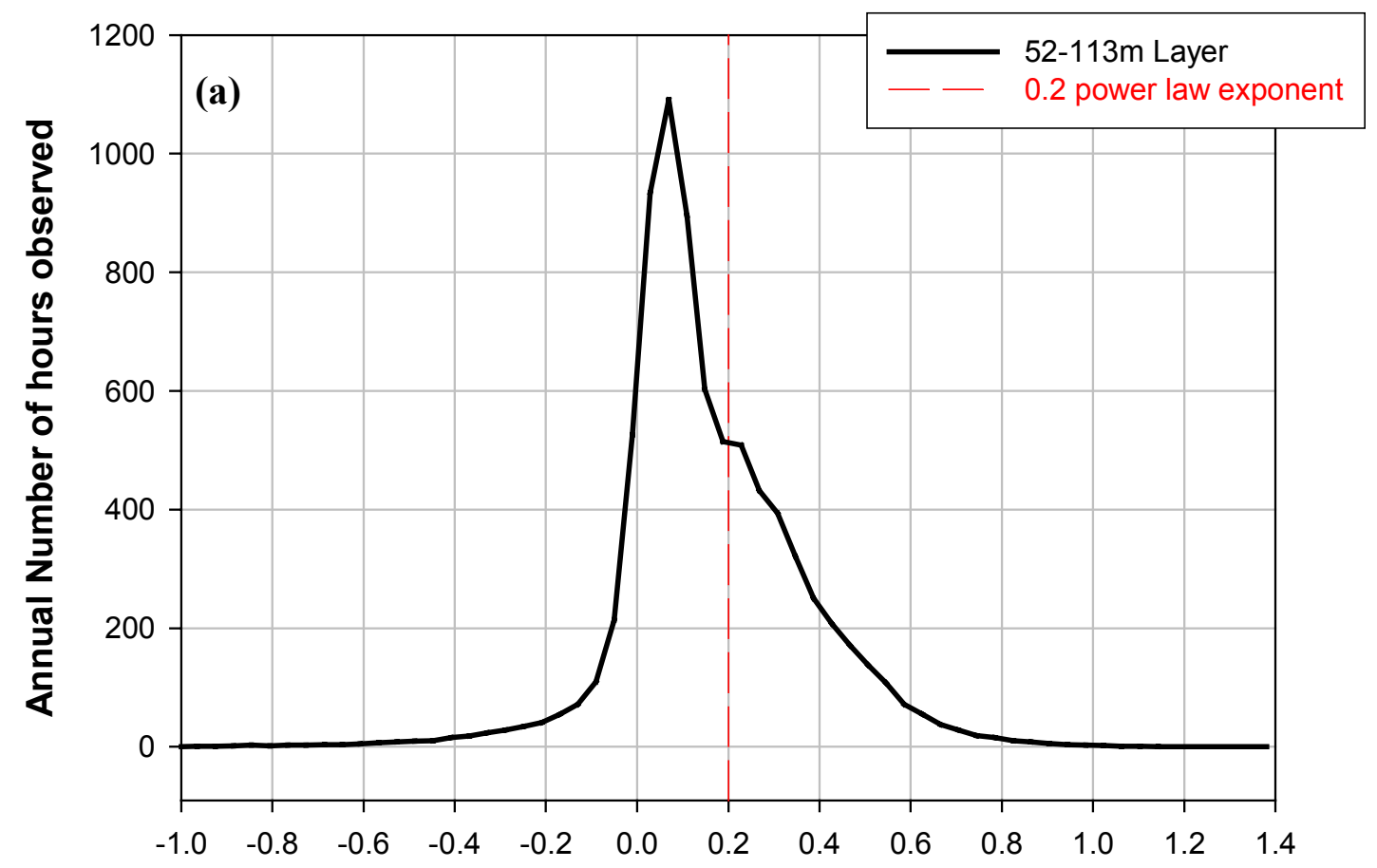

5-minute 52-113m layer shear exponent

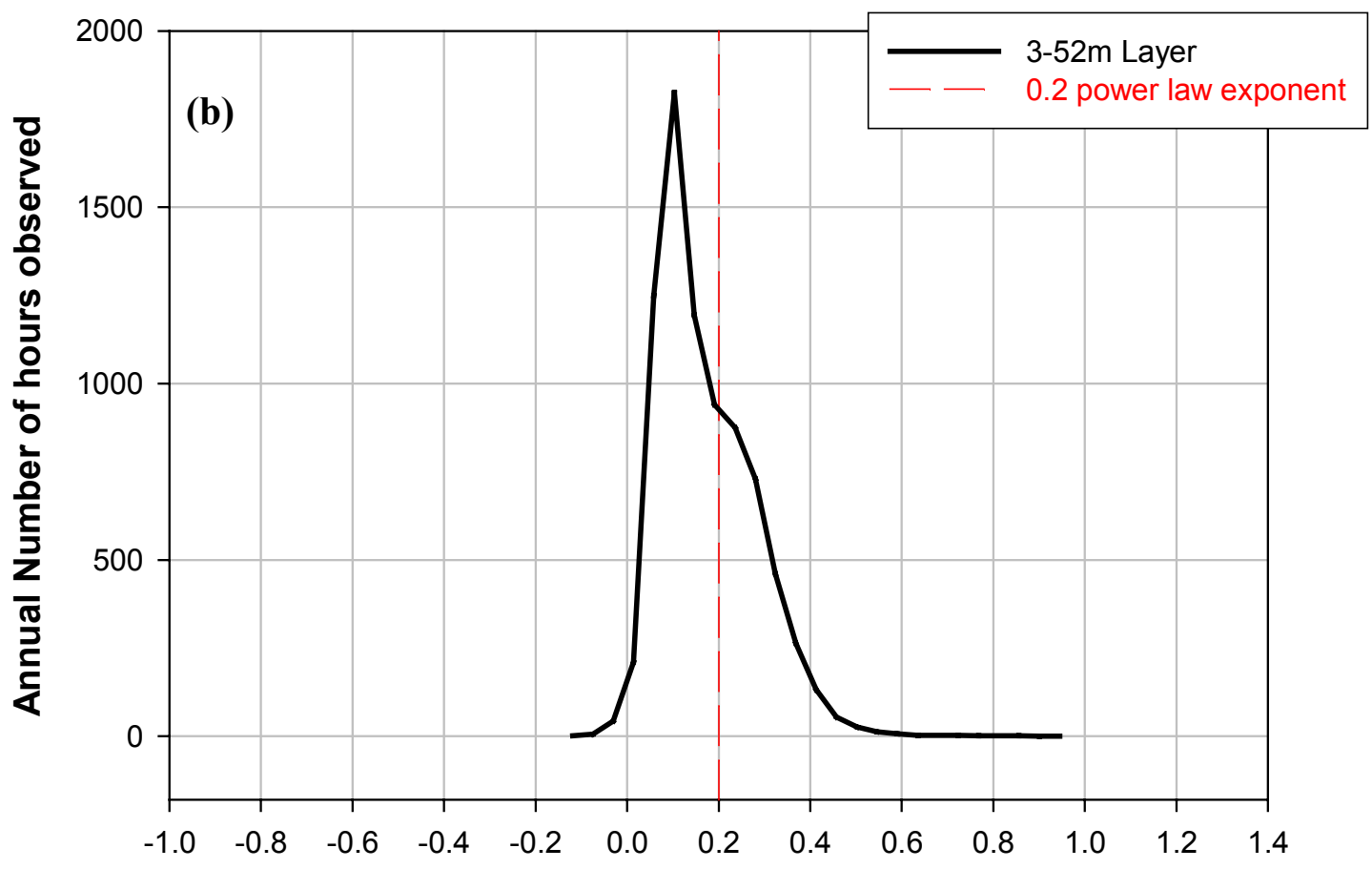

5-minute 3-52m layer shear- exponent

Figure 5-12. Annual probability density of 5-minute layer shear exponents at the (a) 52- to 113-m layer, (b) 3- to 52-m layer 

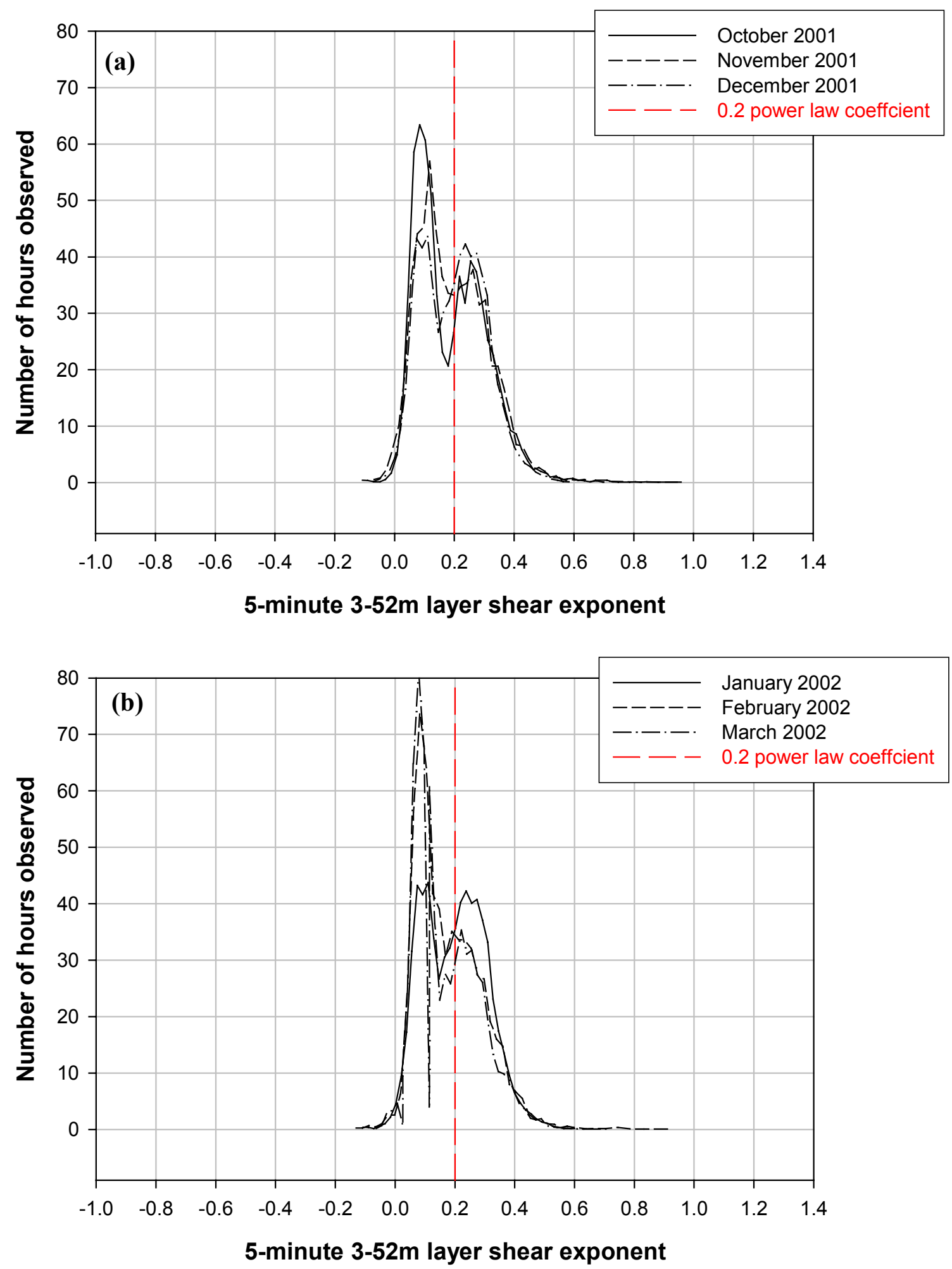

Figure 5-13. Cold season (October-March) probability density distributions of shear exponents at the 3- to 52-m, 5-minute layer: (a) Oct-Nov-Dec, (b) Jan-Feb-Mar 


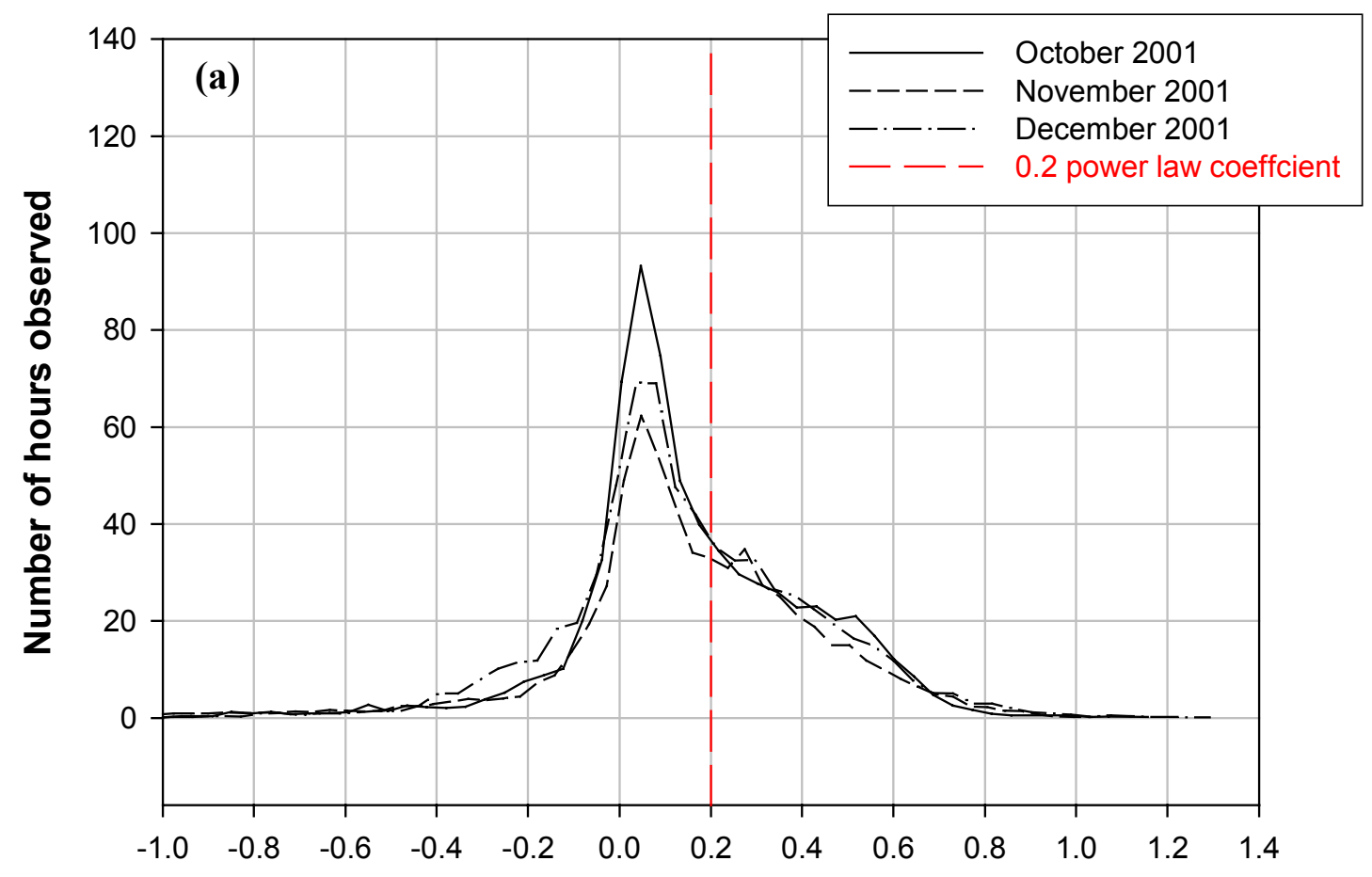

5-minute 52-113m layer shear exponent

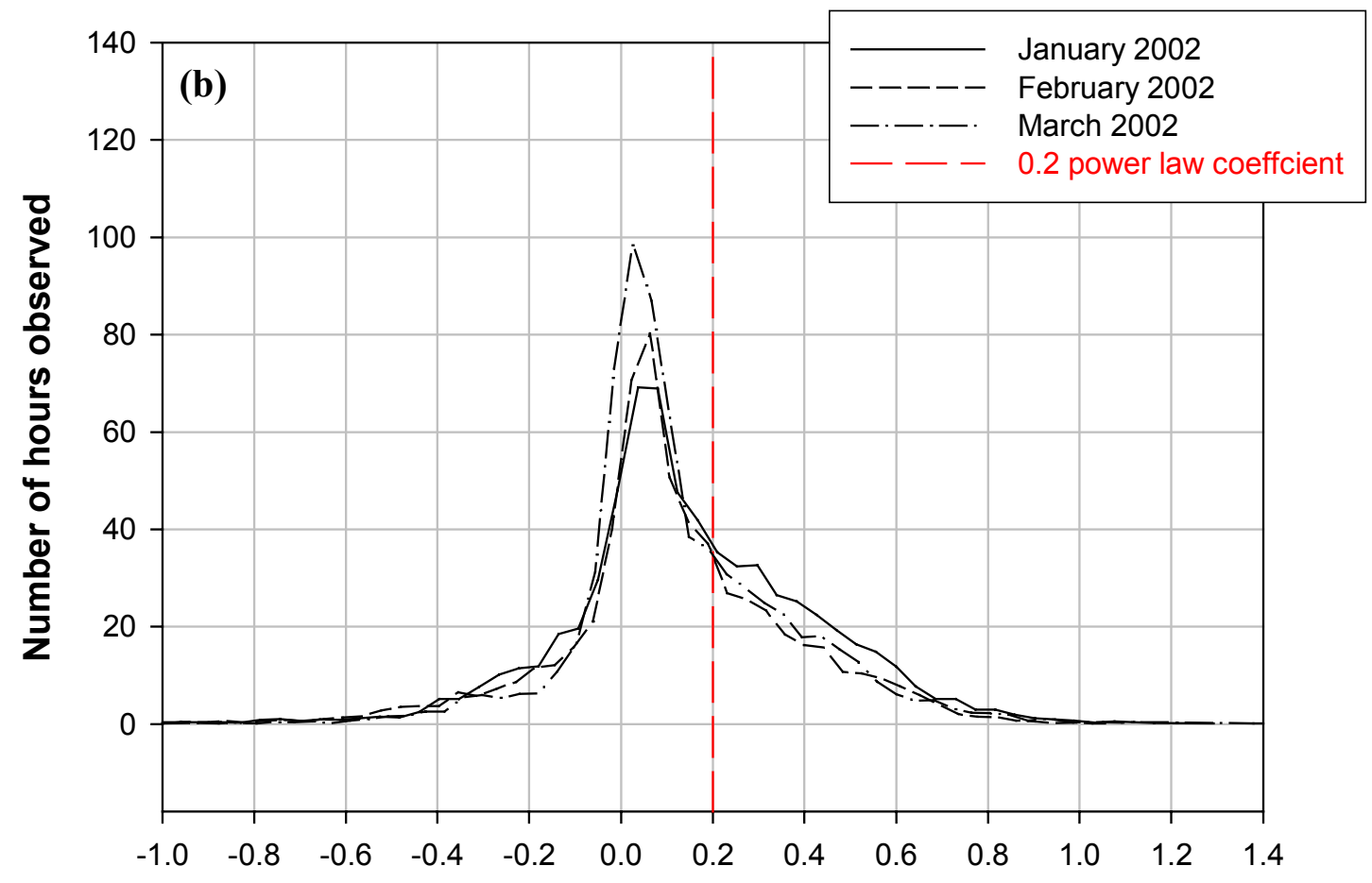

5-minute 52-113m layer shear exponent

Figure 5-14. Cold season probability density distributions at shear exponents at the 52- to 113-m, 5-minute layer: (a) Oct-Nov-Dec, (b) Jan-Feb-Mar 

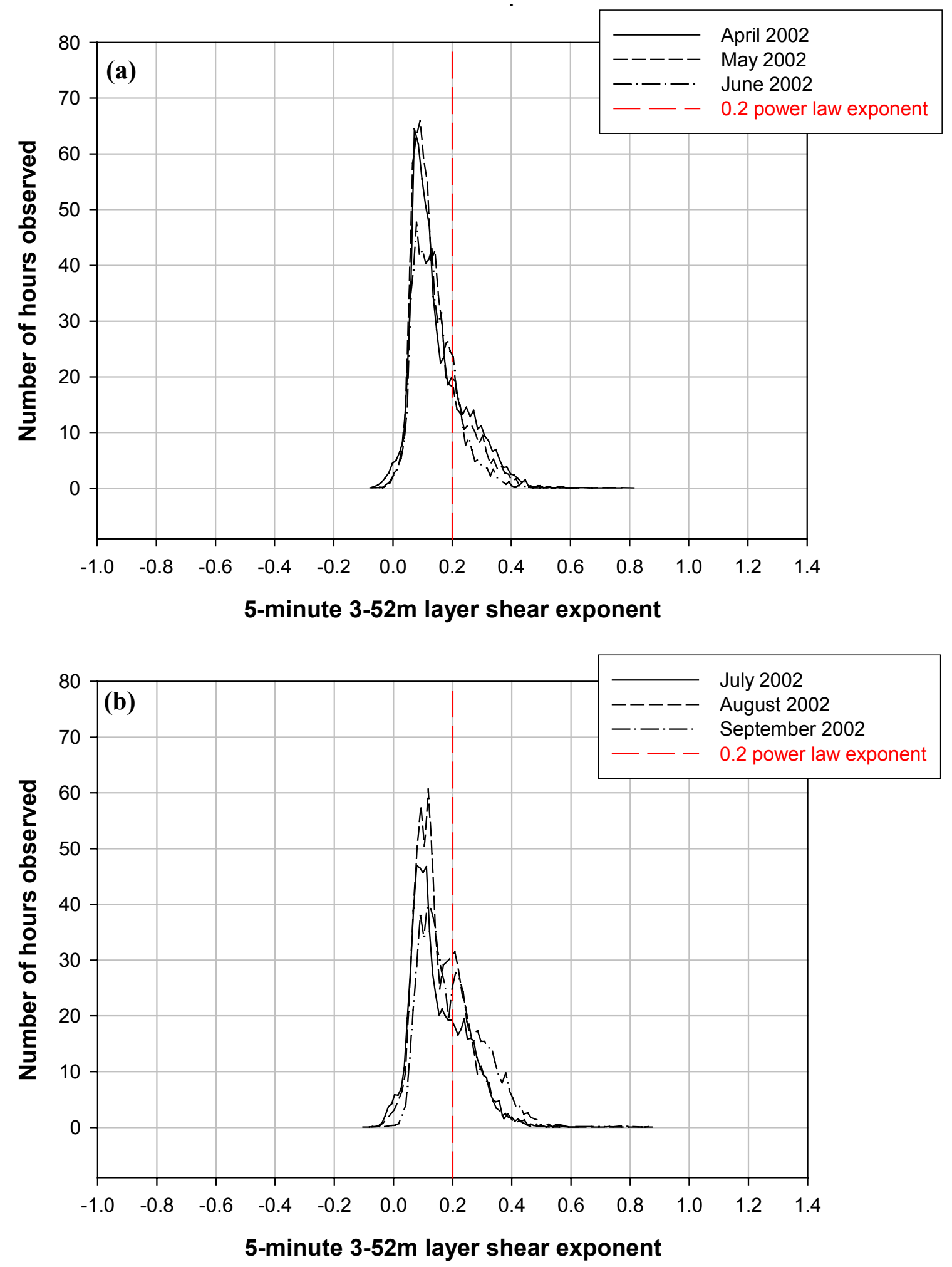

Figure 5-15. Warm season (April-September) probability density distribution of sheer exponents for the 3- to 52-m, 5-minute layer: (a) Apr-May-Jun, (b) Jul-Aug-Sep 


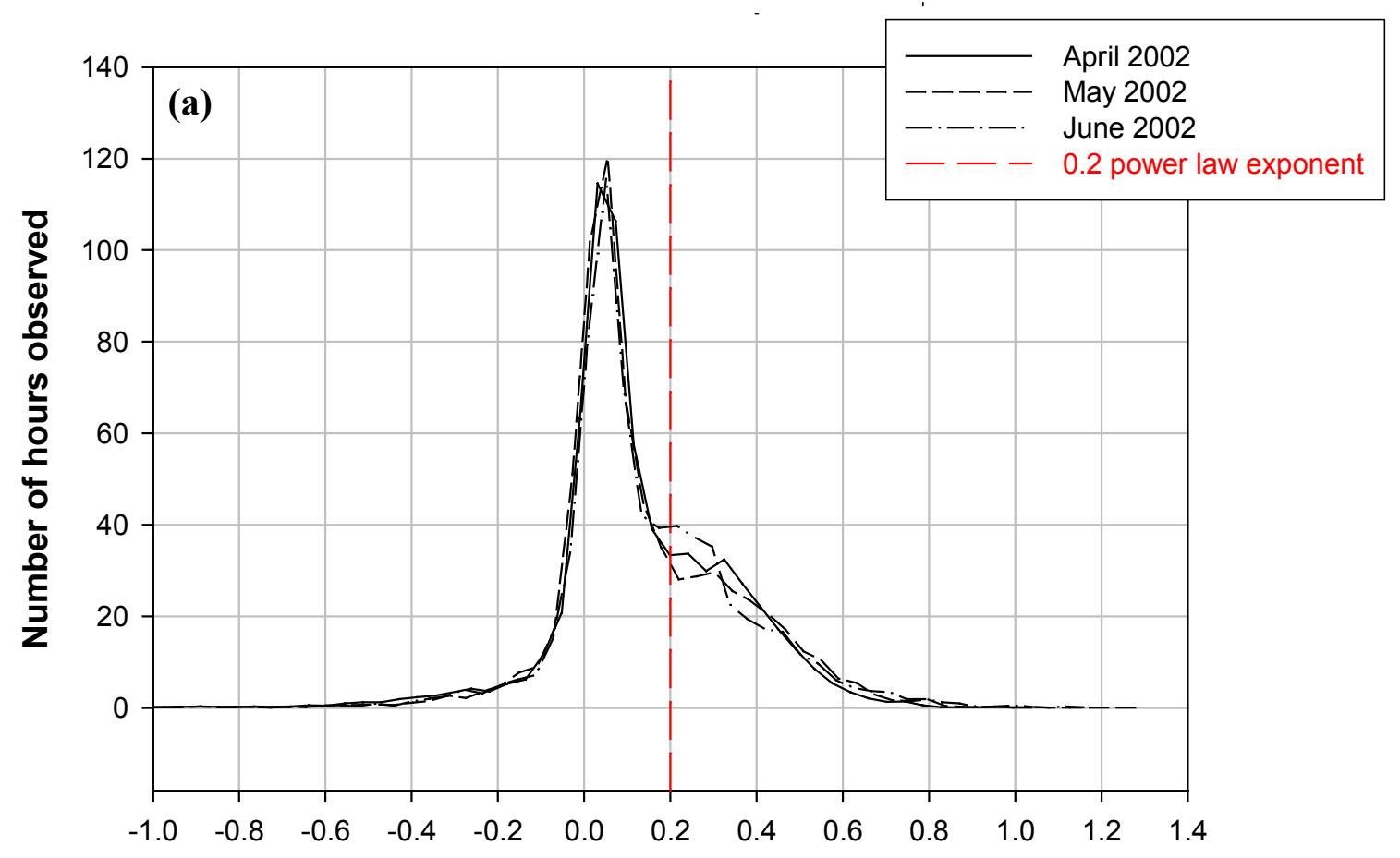

5-minute 52-113m layer shear exponent

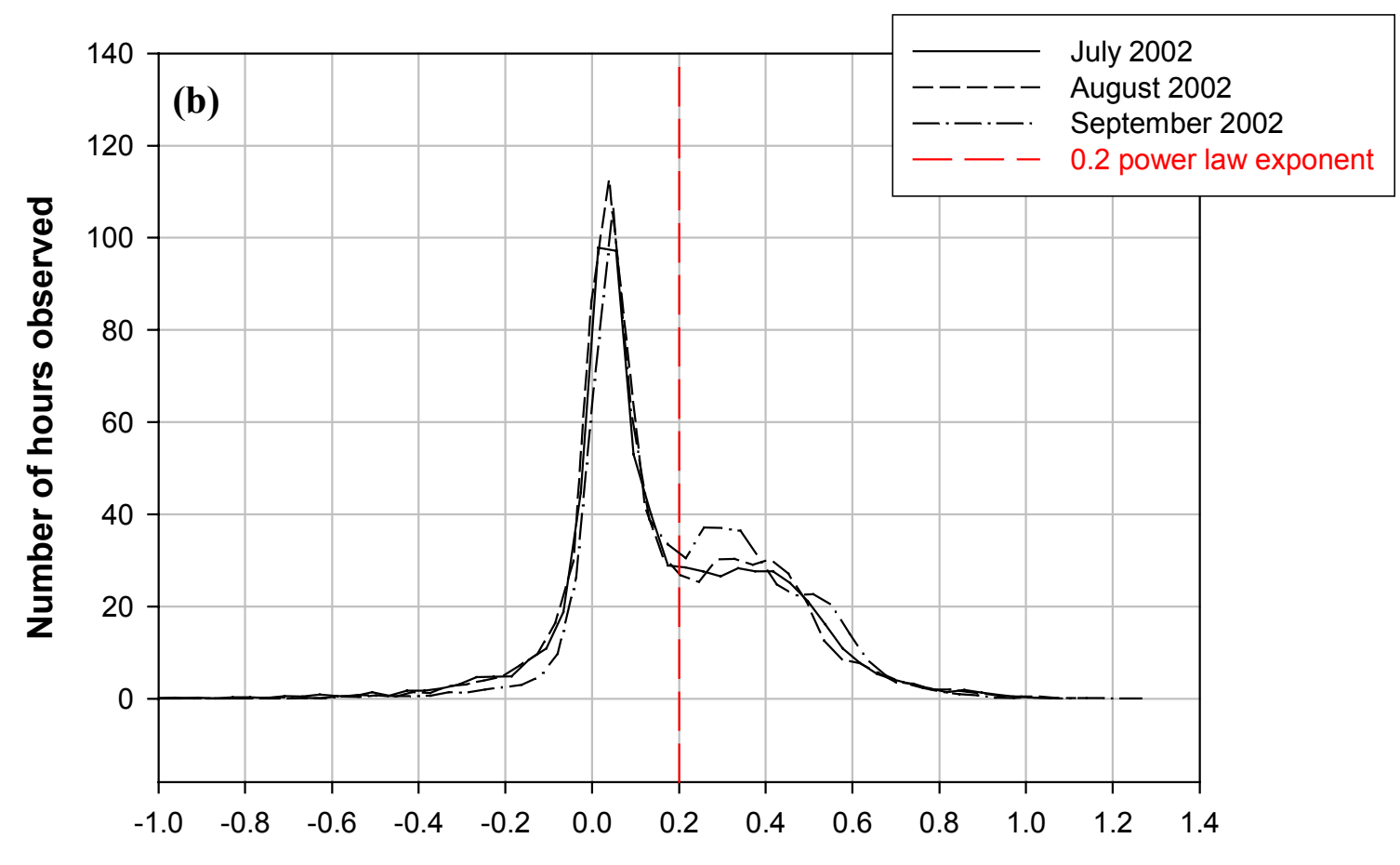

5-minute 52-113m layer shear exponent

Figure 5-16. Warm season probability density distributions of sheer exponents at the 52- to 113-m, 5-minute layer: (a) Apr-May-Jun, (b) JulAug-Sep 


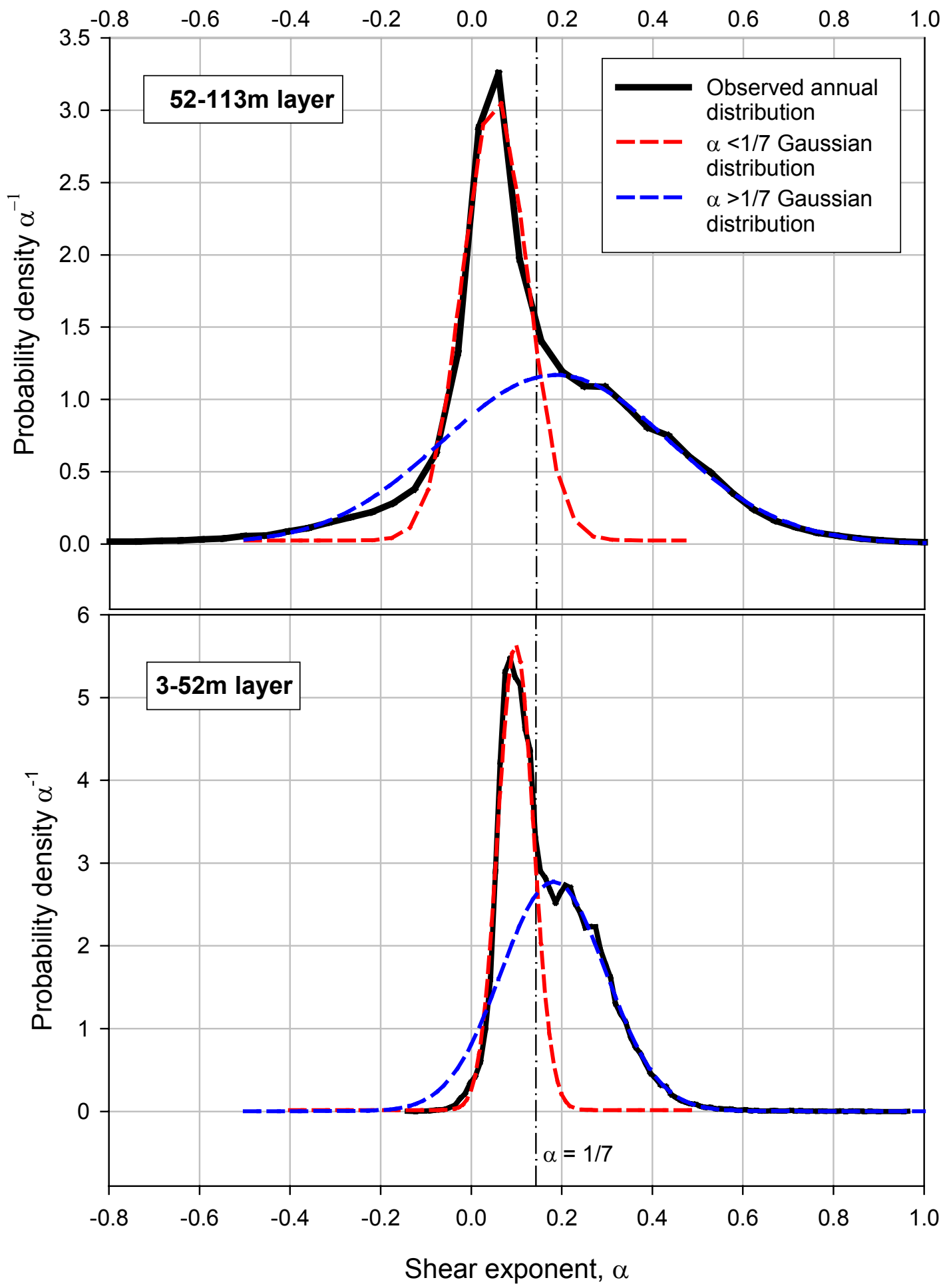

Figure 5-17. Gaussian fits of annual shear exponent probability density distributions 

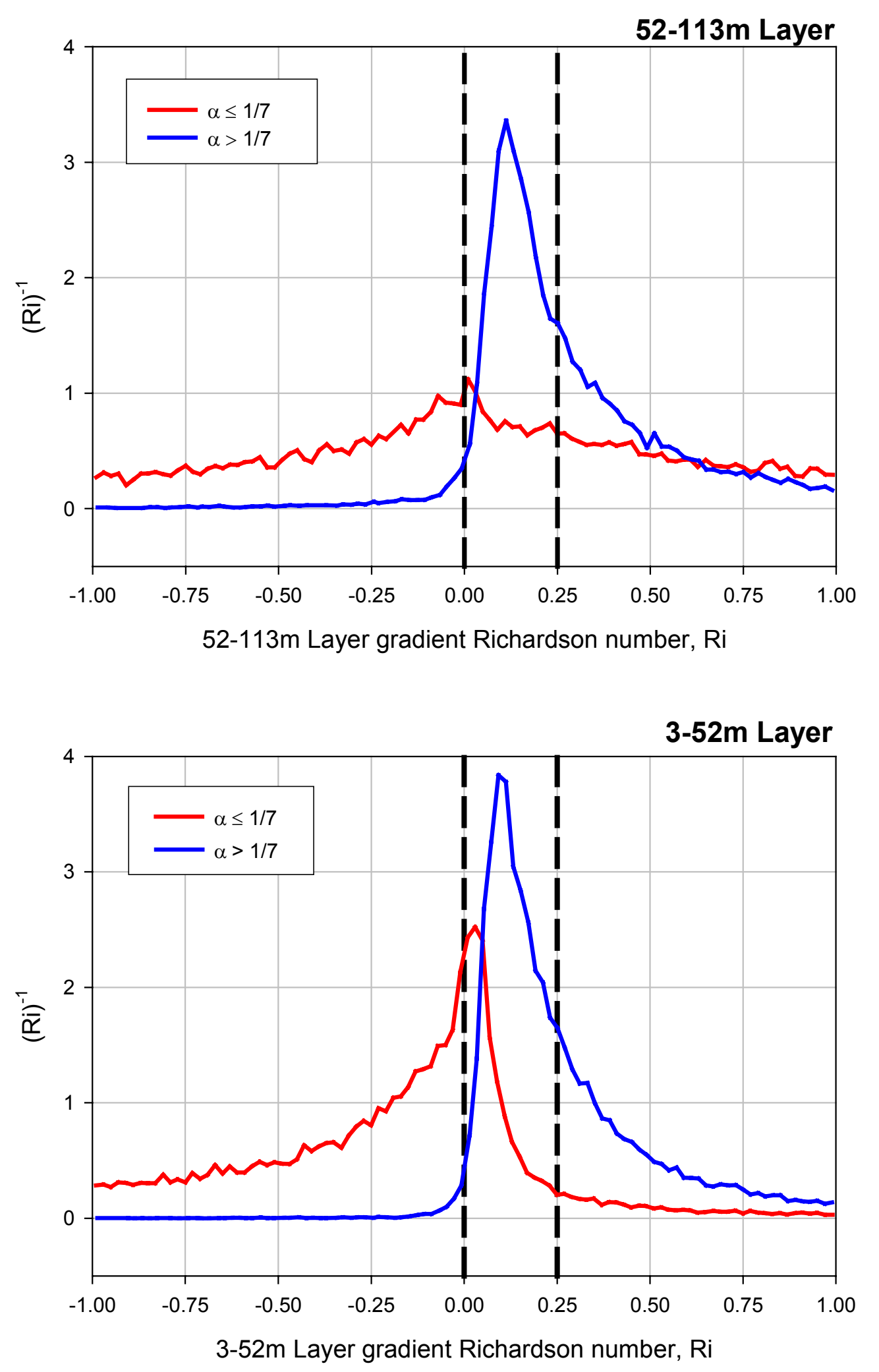

Figure 5-18. Probability density distributions of shear exponent distributions as a function of class and stability (Ri) 


\section{Cumulative Hours of 52m Mean Wind Speed when 52-113m Layer Shear Coefficient $>0.2$}
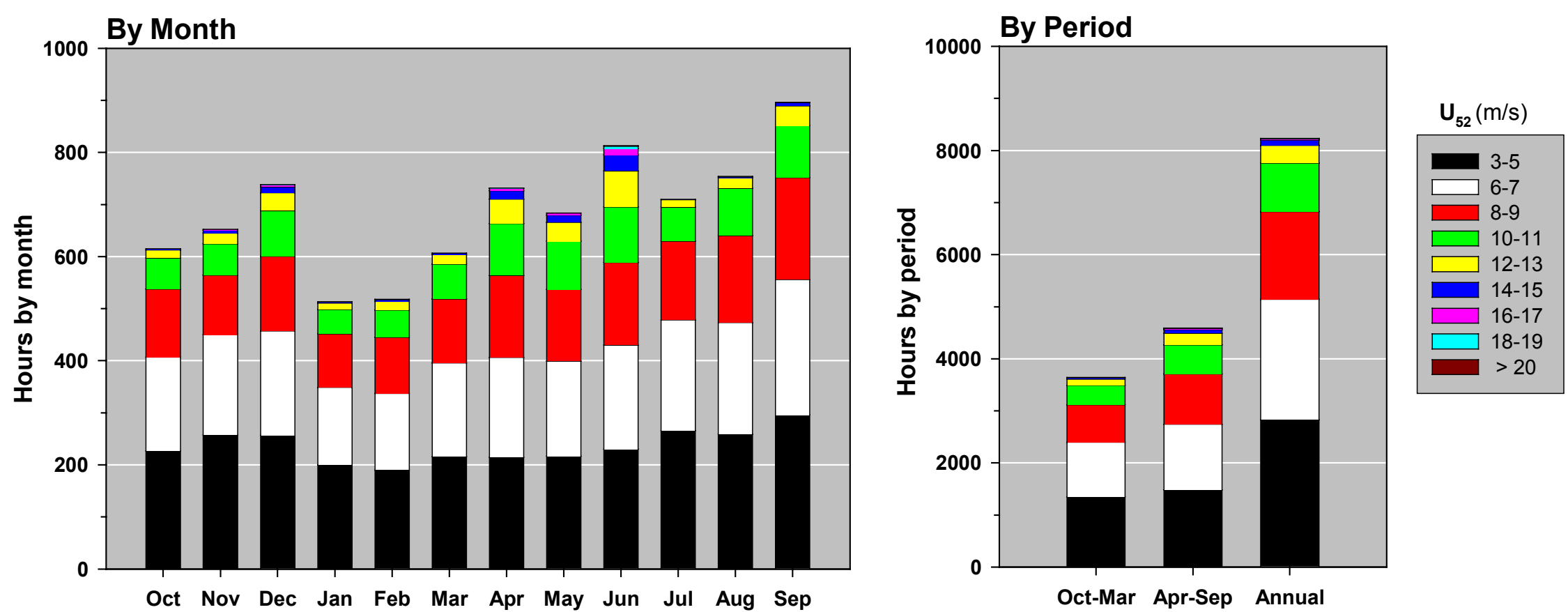

Figure 5-19. Cumulative hours of mean wind speed at 52-m when the 52- to 113-m layer shear exponent is $>0.2$ 

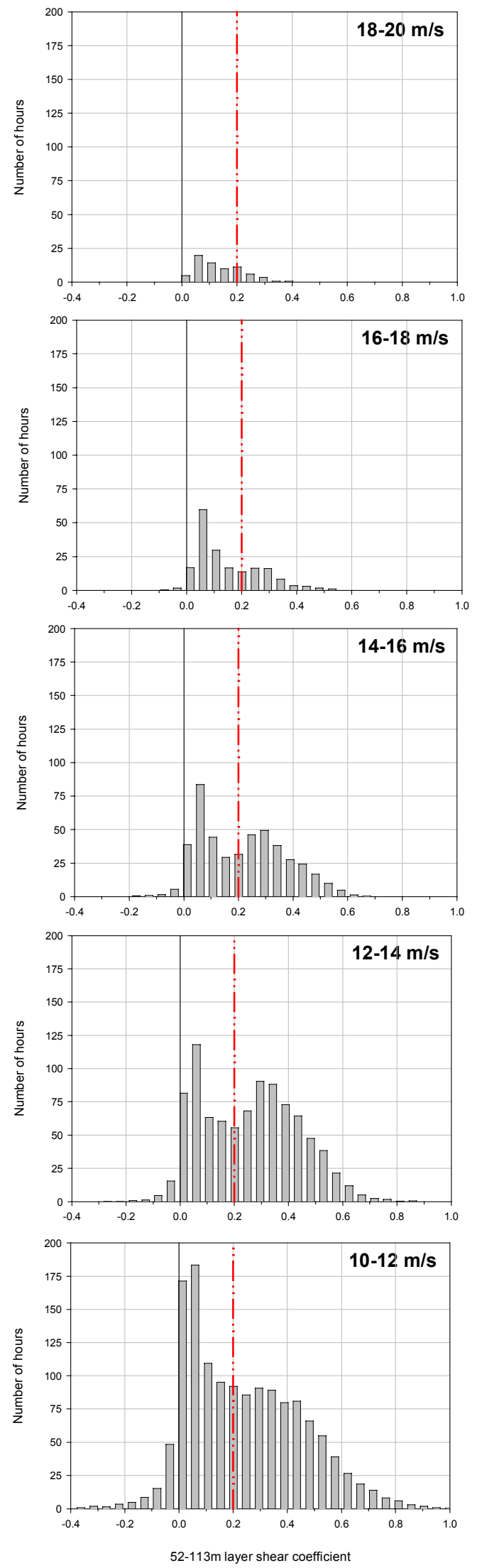

Figure 5-20. Annual distributions in hours of the sheer exponent at the 52- to 113-m layer as a function of the mean wind speed at 52-m 

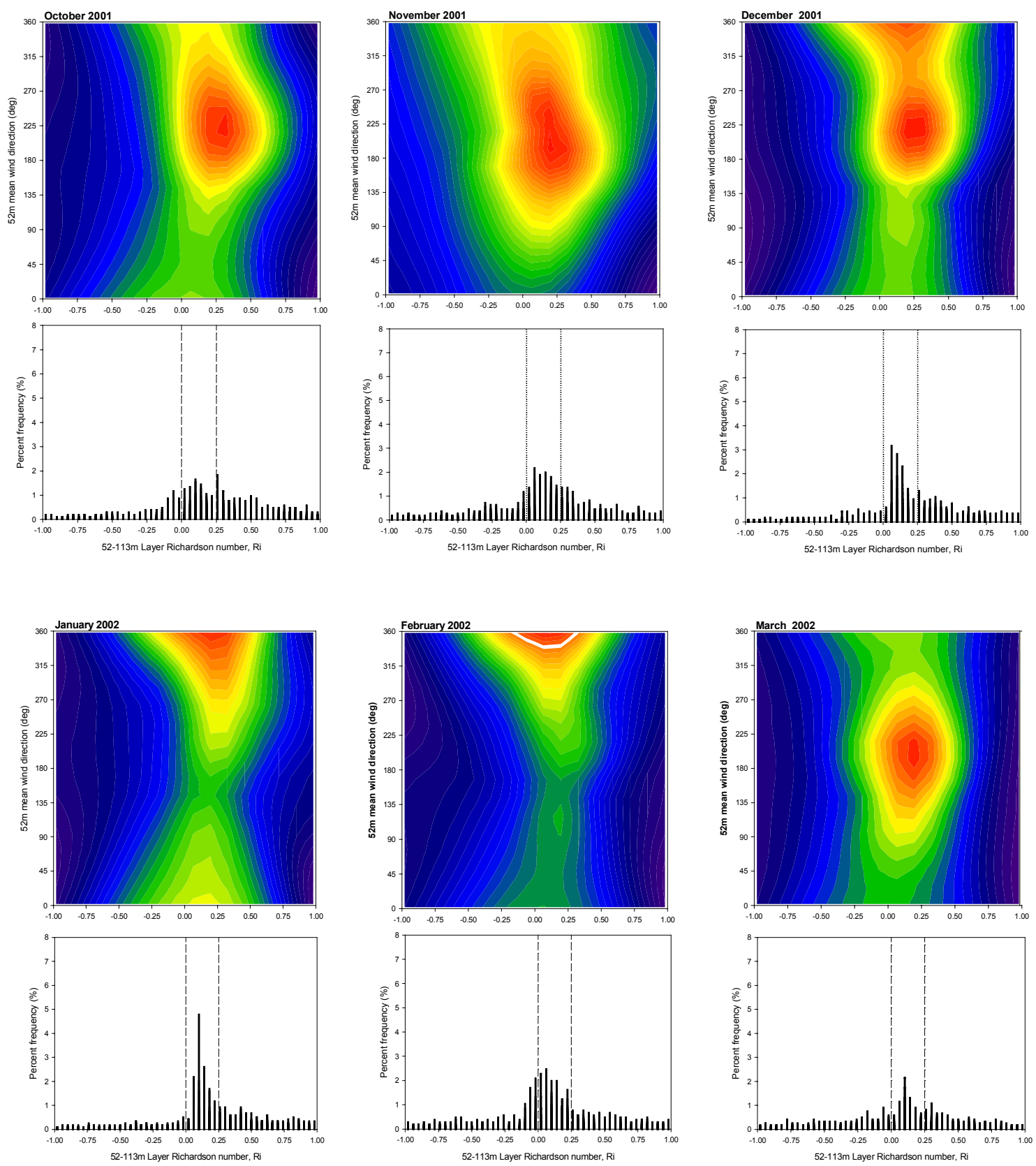

Figure 5-21. Cold season monthly joint probability distributions of the mean wind direction at 52-m and the gradient Richardson number (Ri) at the 52- to 113m layer, plus monthly frequency distributions of $R i$ 

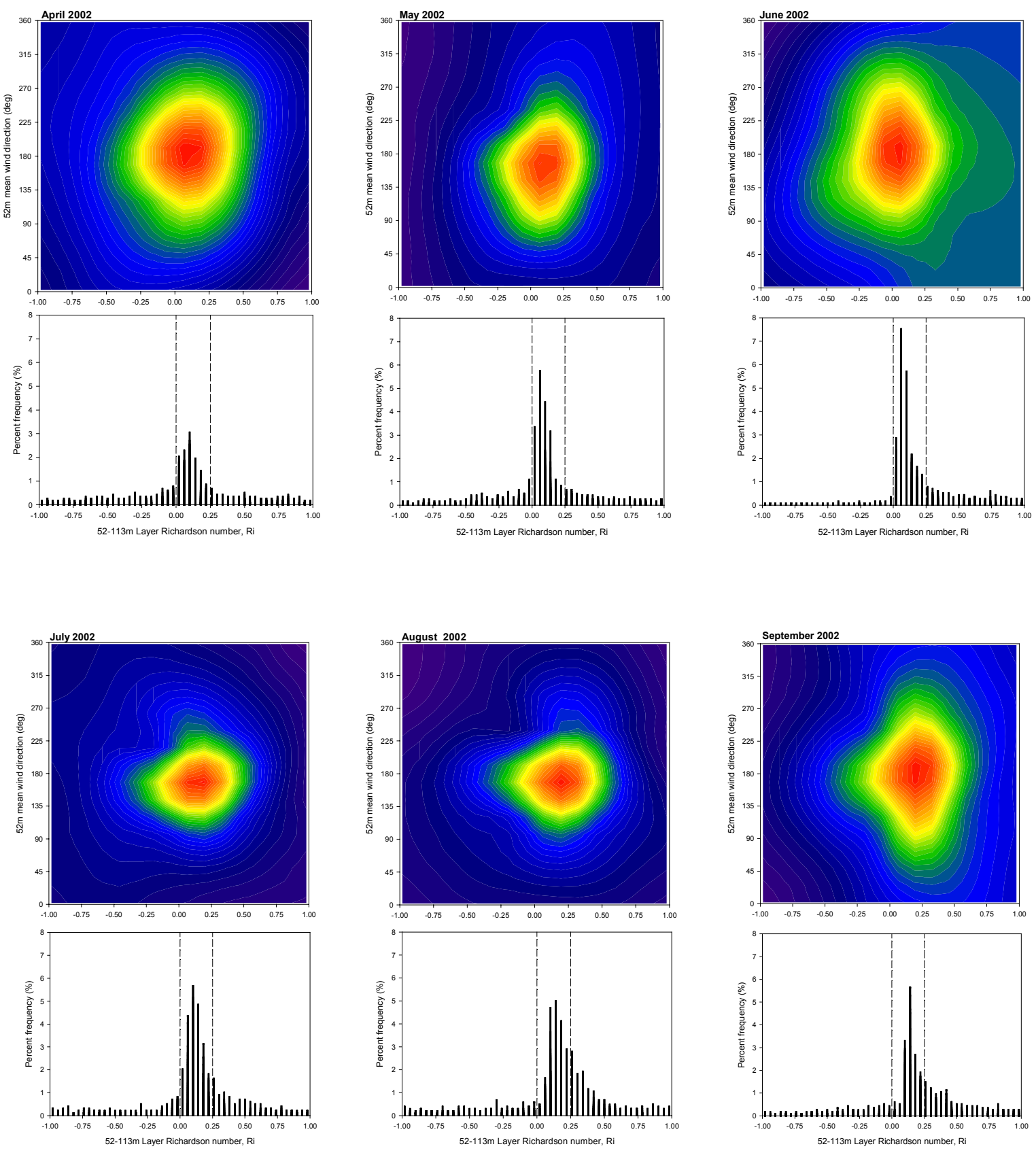

Figure 5-22. Warm season monthly joint probability distributions of the mean wind direction at 52-m and gradient Richardson number $(R i)$ at the 52- to 113-m layer, plus monthly frequency distributions of $R i$ 

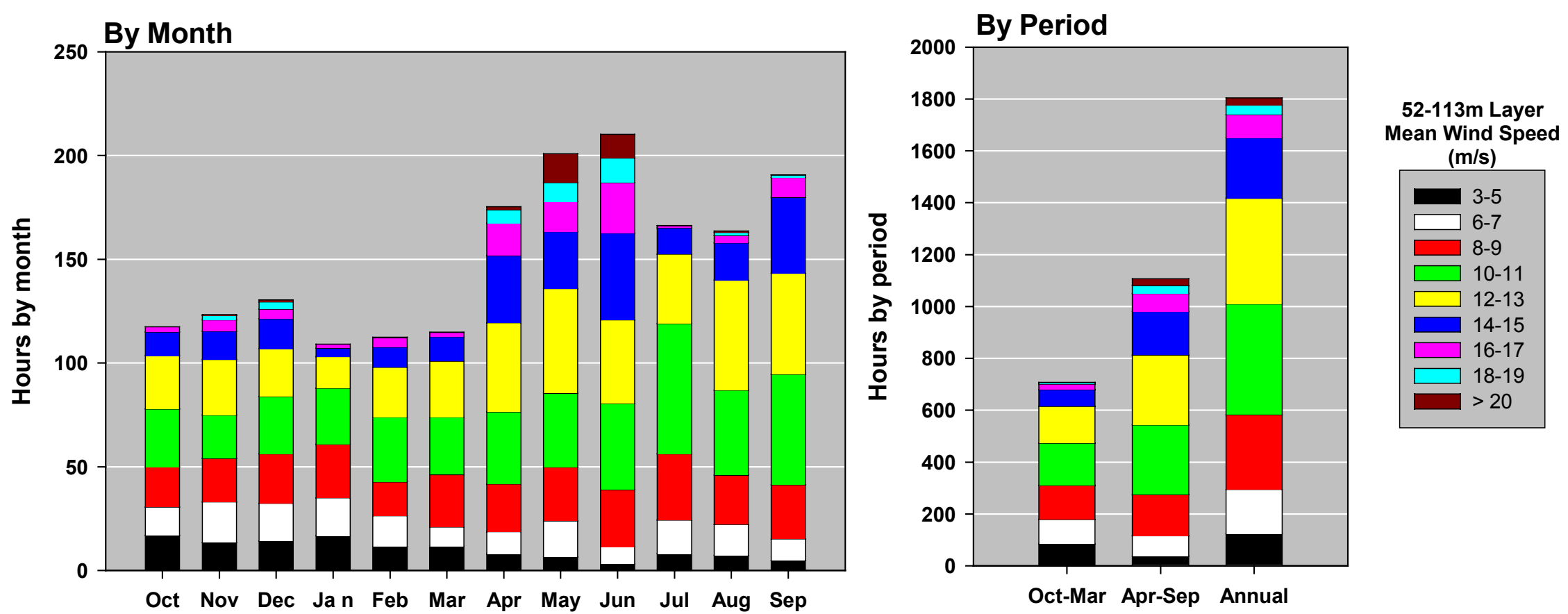

Figure 5-23. Cumulative hours of the mean wind speed at the 52- to 113-m layer when $R i$ is within critical range $(0<R i<0.25)$ 

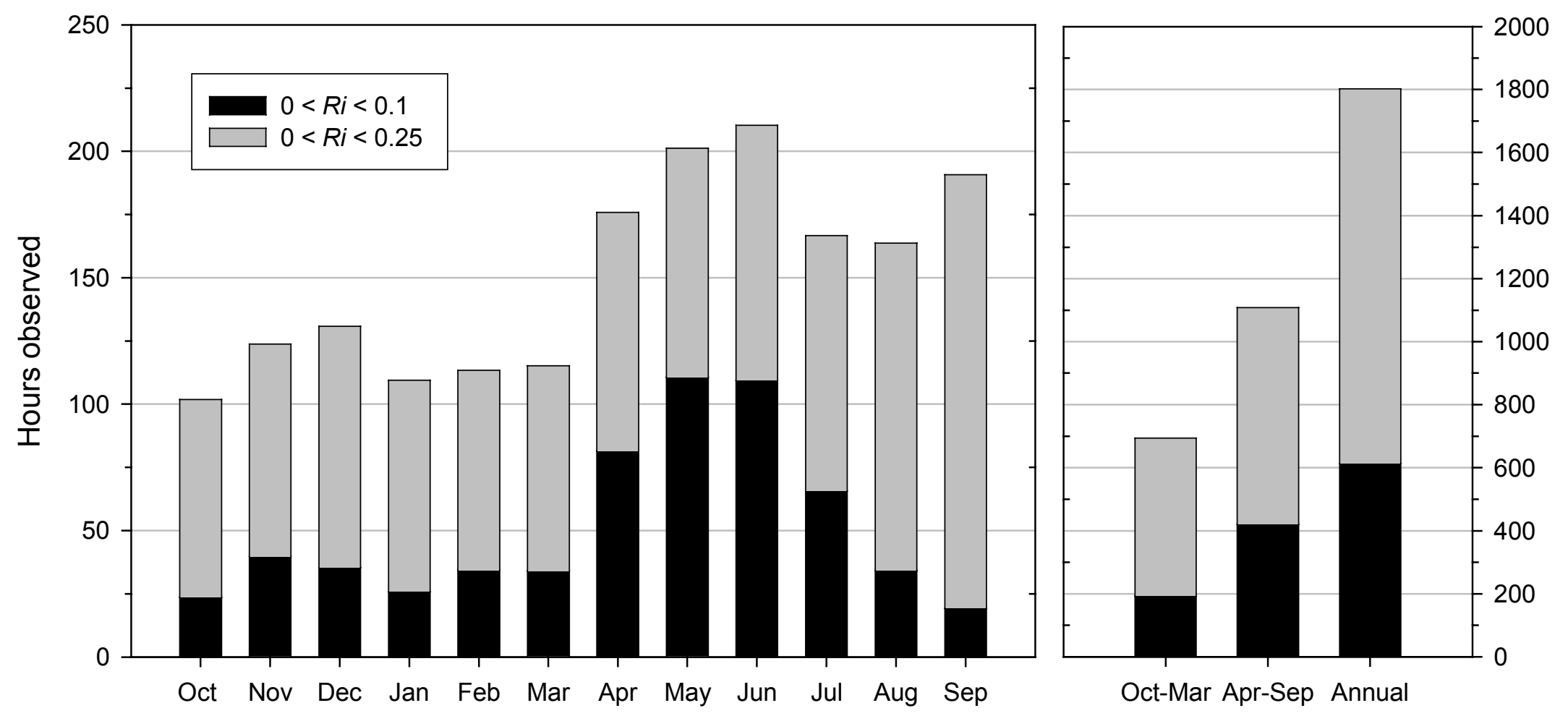

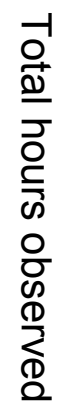

Figure 5-24. Observed monthly critical stability conditions distributions in the 52- to 113-m layer when mean wind speed at $52-\mathrm{m}$ is $>3 \mathrm{~m} / \mathrm{s}$ 


\section{Three Representative Case Studies}

In this section, we discuss what we believe are three representative cases studies collected during nocturnal boundary layers in June 2002. We will use the results of these studies to design the final data-processing procedures that will be applied to the entire 1-year record of more than 30,000 10-minute records of high-speed, detailed turbulence information collected between March 2002 and March 2003. The cases analyzed were chosen on the basis that they represent commonly occurring sequences of conditions that we have observed, and included reasonably complete SODAR wind profiles.

We believe that the meteorological conditions present within these study periods and those similar to them will be frequently encountered by future turbine operations not only at this site, but elsewhere, at least in the western Great Plains where low-level jets are known to form. They embody conditions associated with warm season (April through September) jet formation (i.e., predominately occurring with southerly winds and evidence of inertial oscillations influencing the heights and magnitudes of jet maxima and the severity of the vertical shears and turbulence conditions beneath them). The details associated with cold-season northerly jets and their influence on shear and turbulence conditions at the heights occupied by current and larger wind turbines (LWST) will be investigated. The loss of the acoustic wind profiler (SODAR) between essentially November 2002 and April 2003 for repairs limited the data set. Hopefully, enough information can be gleaned from available cold-season measurements and, when coupled with improved SODAR signal processing, will allow us to at least to identify several useable casestudy sequences for detailed analysis.

The three case studies discussed below consist of episodes in which a LLJ was present. The jet does the following:

1. Creates strong vertical shears in the layer below (including the rotor layer) after local midnight, but remains stable and does not breakdown into intense, coherent turbulence (June 17, 2002, AM case)

2. Occurs during the day-night transition period after local sunset, which then suddenly breaks down into two periods of energetic, coherent turbulence lasting 3 hours and then subsides after midnight (June 17, 2002, PM case)

3. Reaches its peak velocity before local midnight, but later decays into long-period wave motions that reach all the way to the surface, which create short bursts of coherent turbulence at the height of the future wind turbine rotors (June 23, 2002, AM case).

In each case, we will discuss the evolution of the LLJ and its influence on the shear and turbulence environment that would have been encountered by a GE $1.5 \mathrm{~S}$ turbine (and the ART turbine if its hub were at the equivalent height). Where appropriate, we discuss the interpretation of the vertical structure of the boundary layer and its influence on the observed shear and turbulence in the turbine rotor layer created by atmospheric wave motions, such as those associated with $\mathrm{KH}$ instability. Finally, in order to assess the potential severity to turbine operations at this location, we aggregate the conditions seen with these three cases and use our simple linear regression model of the ART root flapwise statistical response to estimate the reaction of this rotor if it were placed in similar circumstances. 


\subsection{June 17, 2002 - AM Case}

The first case involves the formation of a LLJ that, while it induces intense vertical shear below a maximum rotor height of $120 \mathrm{~m}$, it did not break into intense coherent turbulence (except briefly at the beginning of the observing period). We first discuss the jet evolution and then its impact on wind turbine operations. All time references are expressed in local standard time (LST) and all heights are above ground level (AGL).

\subsubsection{LLJ Morphology}

The period of interest for this case study was 00 to $04 \mathrm{~h}$ on June 17, 2002. A vector wind profile derived from the Scintec SODAR and display software is shown in Figure 6-1. Here the length of the arrows represent the 10-minute mean wind speed and the orientation of the arrow is parallel to the mean wind direction, while the arrow itself points toward the direction the wind is blowing. Immediately after midnight, the winds are near southerly from the height of the turbine rotor disk up to $400 \mathrm{~m}$, while increasing slowly. At about $01 \mathrm{~h}$, there is a noticeable increase in the wind speed and a shift toward a more southwesterly direction above about $250 \mathrm{~m}$. The speed continues to increase along with a gradual shift of wind direction to the southwest. The speed increase continues to move lower until it reaches the neighborhood of the upper limits of a GE Wind turbine rotor $(\sim 120 \mathrm{~m})$. Two hours after local sunrise (which was 4:29 h), the wind speeds above $120 \mathrm{~m}$ gradually become more westerly and begin to diminish. Finally, after $07 \mathrm{~h}$ the speeds decrease rapidly.

Details of the wind flow up to $500 \mathrm{~m}$ are shown as contours of SODAR-derived wind speed in Figure 6-2, and details of wind direction are pictured in Figure 6-3. The latter clearly defines the evolution of the jet maximum as it descends to a height of about $225 \mathrm{~m}$ after $2: 40 \mathrm{~h}$ and apparently begins to rise again about $04 \mathrm{~h}$. Figure 6-2 also shows that there was a brief maximum of winds from the south $\left(190^{\circ}\right)$ at a height of about $250 \mathrm{~m}$ that existed for about an hour between midnight and $01 \mathrm{~h}$ when it was displaced by the more energetic jet descending from above as the winds shifted to the southwest. The rapid veering of the wind to the southwest (Figure 6-1) is even more dramatic in Figure 6-3. The flow in the whole layer depth of 50 to 500 $\mathrm{m}$ became southwesterly by $3: 30 \mathrm{~h}$. This sequence of events clearly demonstrates the process of warm-season jet formation described by Whiteman et al. [16] and is likely based on the dynamics explained by Blackadar [18] (i.e., the role of the inertial oscillation as a key element). The effects of the oscillation-induced accelerations are mixed out as daytime convectional heating becomes well established after about $07 \mathrm{~h}$.

\subsubsection{Vertical Shear Characteristics}

The development of vertical shear below the LLJ maximum is better described by dividing the entire observational period into three segments. The first segment encompasses the period of the weaker jet and mostly southerly flow up to $1: 10 \mathrm{~h}$, the second included the period when the stronger jet evolved and began its descent (1:20 to 2:30 h), and the final period of 02:40 to $04 \mathrm{~h}$ was when the jet achieved its maximum velocity. The SODAR-derived wind profiles (10-m vertical resolution) that correspond to these three sub-periods are plotted in Figure 6-4. The weaker jet shown in Figure 6-4a reached a peak at 0:40 h. While significant, the shear below $200 \mathrm{~m}$ was not particularly exceptional below the top of an equivalent GE Wind 1.5S rotor (120 
$\mathrm{m}$, the layer outlined by the dashed lines). As the second jet descended in the second segment plotted in Figure 6-4b, the shear increased rapidly by the end of the period above $120 \mathrm{~m}$, but also below it as well. Finally, during the last segment pictured in Figure 6-4c, the shear below the jet velocity maximum reached its peak below $200 \mathrm{~m}$, but it is particularly severe between 120 and $150 \mathrm{~m}$. The entire wind speed profile evolution is presented in Figure 6-5 in which each individual profile has been slightly displaced in order to allow comparisons of the individual shapes.

Figure 6-6 shows the vertical shear profiles expressed in terms of shear exponents and calculated from the SODAR data over 20-m height increments below the nominal height of the jet maximum $(\sim 220 \mathrm{~m})$ for the three segments. Clearly, the region of strong vertical mean shear gradually descended between midnight and $04 \mathrm{~h}$ when it reached a peak of $\alpha=1.2$ after $03 \mathrm{~h}$ at an elevation of $120 \mathrm{~m}$ or right at the top of a GE turbine rotor. The mean wind speed at this height (Figure 6-4) remained just below rated (about $11.5 \mathrm{~m} / \mathrm{s}$ ), while the speed at the bottom of the rotor varied between 6 and $8 \mathrm{~m} / \mathrm{s}$. Obviously, if larger and taller turbines with rotor extending up to $200 \mathrm{~m}$ were present, they would be exposed to high shears for several hours. The steadiness of the shear below $200 \mathrm{~m}$ is demonstrated in Figure 6-7, which profiles averages for each of the three segments. The temporal details of the shear across a GE Wind 1.5S rotor are shown as shear exponent contours in Figure 6-8. After about $02 \mathrm{~h}$ and for at least the next 2 hours, the turbine experienced increasing shear over the upper half of its rotor, which reached a maximum after $03 \mathrm{~h}$. Currently, IEC specifications call out a design (Normal Wind Profile [NWP]) of 0.2 across the rotor disk. Not only would the criteria be exceeded over the entire disk in this situation, it would vary by more than six fold over that disk height for several hours.

\subsubsection{Turbulence Environment and Potential Turbine Impacts}

A summary of background inflow turbulence scaling characteristics calculated from 10-minute segments using the 120-m tower measurements are shown in Figure 6-9. Figure 6-9a plots the near hub-height wind speed of $85 \mathrm{~m}$ (the actual turbine hub-height is $80 \mathrm{~m}$ ) and the 52- to $113-\mathrm{m}$ layer gradient Richardson number $R i$. With the exception of a brief period at 0:30 h, the mean hub-height wind speed remained below rated throughout the period of interest. The dynamic stability of the layer occupied by the turbine rotor remained stable $(R i>0)$ for the entire period as well. Both the critical $\left(R i_{c}\right)$ and weakly stable $R i$ ranges $(R i<0.25$ and $R i<0.1$, respectively) have been indicated. Except for the period between 1:20 and 1:50 $\mathrm{h}$ when the weaker LLJ was being replaced by the stronger one (Figure 6-2), the rotor layer remained at or within the critically stable range (i.e., $0.25<R i$ ). Only for one brief 10 -minute period at 0:20 h did the $R i$ drop into the weakly stable regime $(0.1<R i)$, the remainder of time it was always larger.

The results of the higher wind speed at the beginning of the study period, when coupled with the observed stability stratification, can be seen in both Figures 6-9b and 6-9c. Both the hub-height vertical wind speed standard deviation $\left(\sigma_{w}\right)$ and peak coherent turbulence kinetic energy $\left(E_{c o h}\right)$ were at their highest values when the weaker LLJ was at its maximum velocity and were accompanied by at- or near-rated hub mean wind speeds and weakly stable conditions. The values of $E_{c o h}$ were above the response threshold seen on the ART turbine and briefly above the significant response level as well, indicating that this turbine (and probably the GE) would likely have reacted with increased flapwise root loads. Also, as indicated in Figure 6-9c, the buoyancy length scale normalized by the GE rotor diameter $\left(l_{b} / D\right)$, while not achieving a value of 1.0 , did climb to 0.6 , indicating that wave motions present would have maximum scale on the order of 
$60 \%$ of the rotor diameter. We found previously that an $l_{b} / D$ ratio of 1.0 or more brought the largest response from the ART turbine. Finally, during this same time the buoyancy frequency $N$ reached its lowest values $(0.05$ to $0.2 \mathrm{cycles} / \mathrm{h})$, indicating that any waves present would have a maximum period of $1200 \mathrm{~s}$, which would allow an ART blade to sample them as many as 800+ times at a rotational rate of $43 \mathrm{rpm}$ (the equivalent for the GE turbine at a nominal $20 \mathrm{rpm}$ would be less than half or 400 samples).

As conditions became more stable after about $01 \mathrm{~h}$ (Figure 6-9), the $l_{b} / D$ ratio, $\sigma_{w}$, and the peak $E_{c o h}$ all decreased, but at the same time $N$ increased (which reduced the maximum period of any waves present). This increase in stability between 50 and $120 \mathrm{~m}$ (particularly after $02 \mathrm{~h}$ ) was accompanied by a corresponding increase in the mean vertical shear (Figure 6-10) as derived from the tower-based sonic anemometer measurements. A close examination of the plots of $\sigma_{w}$ and peak $E_{c o h}$ in Figure 6-9b reveals the same periodicity in both between 1:40 and 3:40 h, with periods of 50, 30, and 45 minutes (an average of about 42 minutes), indicating that relatively long-period internal gravity or buoyancy waves were likely present in the very stable air underneath the LLJ.

Figure 6-11 presents a histogram of the 10-minute mean wind speeds near the bottom, the hub, and the top of an equivalent GE rotor (i.e., 54, 85, and $116 \mathrm{~m}$ ). With the exception of the period of time between about 1:20 and $02 \mathrm{~h}$, the winds at the top of the equivalent rotor height remained at or slightly above rated. Thus, a rotor would have been operating in intense mean shears. In order to estimate what shears the rotor will see dynamically (i.e., during the period of one revolution), we smoothed the sonic-derived horizontal wind speeds over $3 \mathrm{~s}$. This period is nominally the minimum time required for a single revolution of a GE rotor. We then computed the probability densities of the velocity differences seen between the 54- and 116-m levels or a height difference of $62 \mathrm{~m}$. In order to account for the actual rotor diameter of $70.5 \mathrm{~m}$, we rescaled the measured differences by the corresponding diameter (height) ratio.

The results of these difference calculations are shown in Figure 6-12. Here, we subdivided the entire period of interest into five subsequences. During the first sub-period (0:30 to 1:10 h), when conditions shown in Figure 6-9 indicated the likelihood of coherent turbulence being present, the 3-s wind speed difference across the rotor was relatively broad with a mean of about $4 \mathrm{~m} / \mathrm{s}$, but ranging from -2 to $+9 \mathrm{~m} / \mathrm{s}$. During the segment between 1:20 and 1:40 $\mathrm{h}$, when the turbine layer $R i$ exceeded the $R i_{c}$ range upper limit of +0.25 (Figure 6-9), the mean difference remained near $4 \mathrm{~m} / \mathrm{s}$ but the maximum range decreased, with minimum of +1 to a maximum of $+6 \mathrm{~m} / \mathrm{s}$. Between 1:50 and 2:10 h, when the oscillatory maximums in $\sigma_{w}$ and peak $E_{c o h}$ were present, the mean difference increased slightly as the mean shear was also increasing, but the distribution range broadened a bit with a minimum of +0.5 and a maximum of $+8.5 \mathrm{~m} / \mathrm{s}$. Finally, during the remaining two segments, the mean differences continued to increase to reflect the change in the mean shear, but the distributions again became somewhat narrower with the maximum speed difference range for each segment near $7 \mathrm{~m} / \mathrm{s}$.

Thus, after about $01 \mathrm{~h}$, the layer that would have been occupied by a GE $1.5 \mathrm{~S}$ turbine rotor became sufficiently stable to suppress the growth of the scales of coherent turbulence required to induce significant aeroelastic and structural responses in a turbine equivalent to the ART and likely to a GE turbine as well. The presence of a very well defined LLJ at a height as low as 225 $\mathrm{m}$ in this case did not result in a breakdown that created a deep layer of organized turbulence from $\mathrm{KH}$ instability. Apparently, at least within the range of the sonic anemometry on the tower, 
no perturbation with sufficient energy to overcome the level of stability was available to trigger the shear instabilities necessary to lead to bursts of coherent turbulence. While there would have been no significant turbulence-induced loading taking place after the first hour (Figure 6-13), a turbine operating in these conditions would have been subjected to several hours of intense vertical shears that increased with height across the rotor diameter. Larger turbines with rotors extending up as high as $200 \mathrm{~m}$ would have experienced similar shear conditions. Because we do not have direct measurements of the turbulence conditions between 120 and $200 \mathrm{~m}$, we have no way to say with any certainty that instabilities developed and coherent turbulent breakdowns occurred that would have adversely affected these larger machines. Given the intensity of the shear immediately below the jet maximum, that may have happened and it just did not propagate downward because of the increased stability closer to the ground. We intend to use the large body of data collected to determine how frequently situations such as this occur.

\subsection{June 17, 2002 - PM Case}

The second case study discusses the day-night transition that took place during the same day as first. This time a LLJ formed in the late evening hours at roughly the same height as that discussed in Section 6.1, but almost immediately broke down into energetic turbulence that reached all the way to the ground. The situation is similar to the sequence of events we witnessed in the San Gorgonio Pass wind farm in California (i.e., the most energetic, coherent turbulence occurring between roughly 18 to $24 \mathrm{~h}$ local time with the peak between 20 and $22 \mathrm{~h}$ ).

\subsubsection{LLJ Morphology}

The period of interest for this case study was 19 to 24 (00) h on June 17, 2002. A vector wind profile from the SODAR is presented in Figure 6-14. At 19:10 h, the winds were relatively light from the south-southeast and gradually strengthened, while also becoming more southerly. These southerly winds increased significantly after $21 \mathrm{~h}$, all the way from the surface, and then gradually shifted toward the south-southwest, particularly at the higher elevations. The sudden change in the plotted wind vectors starting at 22:20 h and continuing intermittently up to 23:50 h indicated that the SODAR either did not receive sufficient return signals during this period or the processing software could not adequately interpret what it did receive. As we will see, this was a period of very strong, turbulent winds that often is a challenge for a SODAR system to interpret properly.

The details of the flow structure up to $400 \mathrm{~m}$ are shown as contours of SODAR-derived wind speed and direction are presented in Figures 6-15 and 6-16, respectively. Figure 6-15 gives an indication of a weak LLJ formation between 150 and $200 \mathrm{~m}$, starting at about 19:30 $\mathrm{h}$ and continuing until 20:50 h. At this time, the winds suddenly increased, reaching a peak between the heights of 250 and $400 \mathrm{~m}$ at about $22: 20 \mathrm{~h}$, with a secondary peak forming near $250 \mathrm{~m}$ at 23:20 h. It is during this second peak that higher speed winds from a southwesterly direction penetrated all the way to the 50-m level (bottom of turbine rotor).

\subsubsection{Vertical Shear Characteristics}

The development of the vertical shear profiles is again better described dividing the entire observational period into three segments, as shown in Figure 6-17. Not all of the profiles are shown because the SODAR was not able to provide them every 10-minutes. The first profile plotted in Figure 6-17a extended from 19 to 20:10 h. During this period, the measured layer 
went from being well mixed with essentially no shear $(19 \mathrm{~h})$ to actually developing a negative shear (19:10 and 19:30 h). At 19:40 to 20:10 h, the weak LLJ maximum formed near $200 \mathrm{~m}$, with the attendant positive shear below. A disappearance of this jet occurred during the early portion of the second segment in Figure 6-17b (20:20 to 20:40 h), after which the jet restrengthened, reaching a maximum velocity of $25 \mathrm{~m} / \mathrm{s}$ at a height of $250 \mathrm{~m}$ at 21:40 h. Finally, the winds continued to increase after 22:00 h (Figure 6-17c), with the shears decreasing below the nominal LLJ peak velocity near a height of $250 \mathrm{~m}$.

The averaged shear profiles calculated in 20-m height increments for each of the segments are shown in Figure 6-18. Clearly, the highest positive shears occurred below $200 \mathrm{~m}$ as was the case from the previous case study shown in Figure 6-7, but the magnitudes and peak values were less than half. Also, as before, the upper half of the GE 1.5S rotor disk experienced higher shears, though just not as severe. The details of the vertical shear distribution (derived from the SODAR) across the layer in which the GE $1.5 \mathrm{~S}$ turbine rotors will reside are pictured in Figure 6-19. The highest shears occurred in the upper half of the rotor disk up to about $21 \mathrm{~h}$, when the wind speeds were lower. After the increase in wind speeds after $21 \mathrm{~h}$, the shear exponents decreased except for a short period at 22:30 h that was in-between the two LLJ peaks (Figure 6$15)$.

The vertical shear profile after $21 \mathrm{~h}$ became much more homogeneous for the most part, as compared with the previous case pictured in Figure 6-8 because of the higher wind speeds and the vertical mixing that is associated with them. The IEC NWP specification of 0.2 was much more applicable during the period after $21 \mathrm{~h}$. Even here, though, there was evidence that using a constant shear exponent across rotor diameters as large as $70 \mathrm{~m}$ or more is not realistic.

\subsubsection{Turbulence Environment}

A summary of background turbulence scaling characteristics calculated from the 10-minute records collected on the 120-m tower are plotted in Figures 6-20 and 6-21. The sequence of events taking place during this period is essentially opposite of those discussed in Section 6.1. In the early morning case, the flow was initially unstable and became stable, whereas the reverse was true in the evening. There was a marked difference before and after $21 \mathrm{~h}$ (Figures 6-20 and 6-21). Before this time, the flow, as indicated by the 52- to 113-m layer $R i$, was much more stable with values occasionally exceeding the $R i_{c}$ range upper limit, but later it moved into the weakly stable regime with the $R i$ averaging near +0.05 . The $85-\mathrm{m}-$ level horizontal mean wind speed before $21 \mathrm{~h}$ was averaging near the GE-turbine-rated wind speed of $12 \mathrm{~m} / \mathrm{s}$, but increased first to about $19 \mathrm{~m} / \mathrm{s}$ at $21: 10 \mathrm{~h}$ and then again to $22 \mathrm{~m} / \mathrm{s}$ at $22: 40 \mathrm{~h}$ before decreasing an hour later back to $14 \mathrm{~m} / \mathrm{s}$. The turbulence characteristics, as indicated by Figures 6-20b and 6-20c, are also dramatic. The parameters $\sigma_{w}$, peak $E_{c o h}$, and the $l_{b} / D$ ratio all exhibited the two-step increases similar to the mean wind speed. The close relationship between $E_{c o h}$ and $l_{b}$ is shown in Figure 6-21a, which underscores the positive correlation between an increase in levels of coherent turbulence and the scale of buoyancy-driven atmospheric wave motions.

The relationship between the turbulence intensities $\left(\sigma_{U_{H}} / U_{H}\right.$ in percent) measured by the sonic anemometers at the 54-, 67-, 85-, and 116-m levels and the 54- to 116-m layer mean peak $E_{c o h}$ follow closely until the second wind speed increase at $22: 30 \mathrm{~h}$ when only the peak $E_{c o h}$ reflects that increase (as does $\sigma_{w}$ in Figure 6-20b). This suggests that the turbulence intensity parameter is not sensitive to the entire range of turbulent flow characteristics being indicated by $\sigma_{w}$ and the 
layer mean peak $E_{c o h}$, both of which were shown previously to highly correlate with the root flapwise bending load response of the ART turbine. This is most likely because the turbulence intensity parameter does not take into account the vertical component of the turbulent motions.

The relationships between the 52- to 113-m layer $R i$ and the 54- to $85-\mathrm{m}$ and $85-$ to $116-\mathrm{m}$ vertical shear exponents are plotted in Figure 6-21c. Before $21 \mathrm{~h}$, when the flow through the rotor layer exhibited greater stability, the shear exponents reached values of 0.6 or more even with near-rated wind speeds. After $21 \mathrm{~h}$, when the wind speeds increased and the flow became weakly stable, the shear exponents dropped to values near the IEC NWP specification $(0.2)$ or lower. This indicates that intense vertical mixing was taking place even though the 54- to 113-m layer $R i$ was still positive $(+0.05)$. For reference, in Figure 6-22a we plotted a histogram of the mean hub-height wind speed estimated from the 67 - and $85-\mathrm{m}$ sonic-derived velocities. Below that in Figure 6-22b, we plotted histograms of the shear exponents corresponding to the 54- to 85-m layer (GE rotor lower half), the 85- to 116-m layer (GE rotor upper half), and the entire 54to 116-m layer (nominally the entire GE rotor depth). Finally, in Figure 6-22c we plotted histograms of the 54- to $85-\mathrm{m}$ and $85-$ to $116-\mathrm{m}$ layer $R i$. The shear between what would be near the bottom and hub of a GE $1.5 \mathrm{~S}$ rotor was always less than between the equivalent hub-height and the rotor top; this is a situation we have seen previously. After $21 \mathrm{~h}$, the shear difference between the lower and upper portions of the rotor became much smaller, reflecting the degree of vertical mixing taking place. Of major importance was the change in layer stability indicated by Figure 6-22c after $21 \mathrm{~h}$. Here we see that after this time, while the upper layer becomes weakly stable, the lower one becomes convective (i.e., $R i<0)$. Thus, in this lower layer there was buoyant turbulence production taking place. We then had a convectively unstable layer actively producing turbulence bounded above with a weakly stable layer that encouraged selective growth of some wave motion scales, while damping others.

In order to understand the situation more clearly, we plotted the mean potential temperature and wind speed profiles (derived from the four sonic anemometers and the cup anemometer at the 3$\mathrm{m}$ height) for the observation period of interest (Figures 6-23a and 6-23b, respectively). Recall that the potential temperature increasing with height indicated statically stable conditions, decreasing with height indicated statically unstable conditions, and constant with height indicated statically neutral. A few vertical dash-dot lines have been provided in Figure 6-23a to help assess the static stability of the three layers (3-54, 54-85, and 85-116 m) shown. All three layers are statically stable (potential temperature increasing with height) following the first two profiles on the left. This remains the condition until $21 \mathrm{~h}$, when the 54- to 85-m layer changed to a negative vertical gradient indicating it has become statically unstable. This layer remained unstable for the remainder of the observation period. The lowest layer (3-54 m) always remained stable (positive gradient), whereas the upper layer also remained stable, but less so (smaller gradient). Thus, we hade a situation where a layer that was both statically and dynamically unstable was sandwiched between two stable layers; the upper layer allowed selective turbulence scale growth and decay. The corresponding layer-mean wind profiles shown in Figure 6-23b exhibited positive shear, though the magnitude of that shear in the two upper layers decreased after $21 \mathrm{~h}$. Thus, all of the ingredients were present for the generation of convective turbulence in what will be the layer occupied by the GE turbine lower rotor disk, which will be damped below. Above the convective layer conditions likely support some form of shear flow instability such as KH instability, which will allow some scales of coherent turbulence to grow while damping others. It is also likely that this layer will damp some scales 
of convective turbulence being generated in the layer below, further concentrating coherent turbulent motions vertically.

We believe that shear flow instabilities resulting in breaking wave motions (such as $\mathrm{KH}$ waves or billows) were responsible for the high levels of coherent turbulent energy and intense vertical motions seen in Figure 6-20. Figure 6-24 plots the mean shear stress profiles

$$
\tau(z)=\rho_{0}(z) \overline{\left[u^{\prime} w^{\prime}(z)\right]}
$$

derived from the four sonic anemometers. The rapid increase in the shear stress at $21 \mathrm{~h}$ suggests rapidly growing wave motions were present because it functions to extract energy from the mean flow and convert this energy into turbulence. The significant peak at 22:30 h involving all four heights indicates the likely presence of strong wave motions throughout the 54- to 116-m (rotor) layer and a probable connection with the peak in $E_{c o h}$ shown in Figure 6-20b. The second stress peak at $23 \mathrm{~h}$, while slightly more intense, is associated with the heights below $116 \mathrm{~m}$.

We now examine the measured turbulence power spectra calculated over of range of $0-2 \mathrm{~Hz}$ of the turbulent wind components $u^{\prime}, v^{\prime}$, and $w^{\prime}$ and instantaneous Reynolds stress components $u^{\prime} w^{\prime}, u^{\prime} v^{\prime}$, and v'w', as well as the probability density distribution of the vertical wind component $w$ ' for records collected at key points in Figure 6-24. For the record collected at 20:40 h, Figure 6-25a plots the $u^{\prime}, v^{\prime}$, and $w^{\prime}$ spectra and the probability density distribution of $w^{\prime}$, while the spectra of the $u$ ' $w^{\prime}, u^{\prime} v^{\prime}$, and $v^{\prime} w^{\prime}$ Reynolds stresses are provided in Figure 6-25b. Two frequency axes have been provided. One is scaled in cyclic frequency up to $1.6 \mathrm{~Hz}$, and the other $\left(f_{n}\right)$ has been non-dimensionalized by the GE turbine rotor diameter $(D=70.5 \mathrm{~m})$ and the mean wind speed at the height of the measurement $\left(U_{H}\right)$. A value of $f_{n}=1$ would correspond to a space scale the size of the rotor diameter, with values of 2, 3, 4, and 5 corresponding to scales of $D / 2, D / 4, D / 4$, and $D / 5$, respectively. This record was chosen to use as a reference for those who follow. The mean wind speed was $12.3 \mathrm{~m} / \mathrm{s}$ (near rated), the rotor layer $R i$ was +0.166 (within the $R i_{c}$ range), and the corresponding shear exponent was 0.566 . The spectral plots show little activity within this frequency band or equivalent space range, and the vertical gusts remain within $\pm 2 \mathrm{~m} / \mathrm{s}$.

The remaining records presented in Figures 6-26 through 6-29 include the rounded shear stress peak in Figure 6-24 at 21:30, the first sharp peak at 22:30, the second peak at 23:00, and minimum at 23:40 h. The spectra and $w$ ' probability density function (PDF) of Figures 6-26a and 6-26b indicate increased turbulence activity with some indication of coherent turbulence present in the $v^{\prime}$ and $w$ ' spectra (distinct peaks) and a broadening of the range of the vertical velocities $w^{\prime}$. Examining the first shear stress peak at 22:30 h (Figures 6-27a and 6-27b), the turbulence levels have increased with the presence of discrete spectral peaks in the Reynolds stresses, particularly in the $v^{\prime} w^{\prime}$ and $u$ 'v' components. There also has been a considerable broadening of the vertical gust velocity range to as high as -8 to $+6 \mathrm{~m} / \mathrm{s}$ at the $54-$ and $116-\mathrm{m}$ heights. The turbulence levels further increase with the second shear stress peak at 23:00 $\mathrm{h}$ (Figures 6-28a and 6-28b) with both the $v^{\prime}$ and $w$ ' turbulence components and all three of the Reynolds stress components showing evidence of discrete spectral peaks out to $f_{n}$ of $4(D / 4)$. The vertical wind gust velocity distribution remains broad, with the ranges associated with the 67- and 85-m levels almost as wide as the others. Finally, at the valley in the shear stress profile at 23:40 $\mathrm{h}$ (Figures 6-29a and 6-29b), the turbulence levels have significantly decreased compared to the previous records at 22:30 and 23:40 h, but there is still evidence of presence of 
coherent turbulence from the fewer and smaller spectral peaks in both the $v$ ' and $w$ ' turbulence components and $v^{\prime} w^{\prime}$ and $u$ 'v' Reynolds stresses. The distribution of the vertical gust velocity remains rather broad, with ranges associated with the 67- and 85-m levels again becoming narrower than those at the 54- and 116-m heights.

We observed that the highest values of $\sigma_{w}$ in the most turbulent records occurred at the lowest and highest measurement levels $(54$ and $116 \mathrm{~m})$ with the two intermediate heights often quite a bit less. This suggests that the whole rotor depth layer may have been subjected to $\mathrm{KH}$ instability after $21 \mathrm{~h}$ because the upper and lower boundaries of KH billows (Figures 2-3b and 2$3 \mathrm{c})$ develop intense small-scale circulations and eventually are the source of shear-gravity waves. The presence of $\mathrm{KH}$ instability may also be the origin of the convective layer between 52 and 83 $\mathrm{m}$ because as $\mathrm{KH}$ billows breakdown, the center region becomes convective as a result of colder, denser air being transported above warmer, lighter air. The persistent convectively unstable layer we see in Figures 6-22c and 6-23a might be the result of the temporal and spatial averaging of these convective circulations within the core of multiple $\mathrm{KH}$ billows forming and decaying.

It is very difficult to isolate turbulence associated with wave motions from the background turbulence field in order to establish the presence of the former. We do know, however, that $\mathrm{KH}$ billows or waves are largely two-dimensional (2-D) structures (at least in their early stages) in an $x$ - $z$ plane of a right-hand coordinate system in which the $x$-coordinate is aligned in the direction of the mean flow. Thus, in this system the streamwise wind component $u$ and the vertical wind component $w$ are parallel to the $x$ and $z$ coordinates. Temporal and spatial periodicities in the $u$ and $w$-components, as well as the correlation between the vertical velocity and temperature fields (usually expressed as the kinematic heat flux or w'T'), can be used as indicators of wave motions in the flow. In Figure 6-30, we plotted the 10-minute variation in height variations of $\sigma_{u}$ and $\sigma_{w}$ for the period of 21 to $00 \mathrm{~h}$, whereas in Figure 6-31 we plotted $\overline{w^{\prime} T^{\prime}}$ for the same levels and time period. An oscillatory behavior throughout the vertical layer is observed in the variations of $\sigma_{u}$ and $\sigma_{w}$, particularly after $22: 30 \mathrm{~h}$, whereas oscillations in $\overline{w^{\prime} T^{\prime}}$ appear throughout the record. The nominal peak-to-peak period for the oscillations shown is on the order of 20-30 minutes; however, the buoyancy frequency $N$ for this period shown in Figure 6-20 was 5-7 cycles $/ \mathrm{h}$, which is a period of 8-12 min/cycle. With the 10-minute resolution of Figures 6-30 and 6-31, we cannot adequately resolve such variations and, therefore, we are probably not seeing shapes of the actual waves involved, but some under-sampled or aliased replica. Finally, we calculated the cross-correlation coefficients between $u^{\prime}, v^{\prime}, w^{\prime}$, and $T^{\prime}$ for each measurement height and presented them in Figure 6-32. Clearly, after $21 \mathrm{~h}$ the correlations in the streamwise and vertical wind components $\left(u\right.$ ' and $\left.w^{\prime}\right)$ and the vertical wind component and temperature strongly suggest the presence of largely 2-D structures in the flow.

The evidence presented indicates that the turbulence environment during this case study period was initially stable with moderate vertical shear. After $21 \mathrm{~h}$, an increase in wind speed set into motion what appears to be the development of some form of shear flow instabilities, such $\mathrm{KH}$ instability that produced lightly damped, rapidly growing wave motions. These motions then became unstable themselves and broke down, creating high levels of coherent turbulence. There was a second resurgence of the process when the wind speeds again increased and the flow became even more turbulent, while reaching all the way down to at least $50 \mathrm{~m}$. The measurements show that during the flow instability period after $21 \mathrm{~h}$, the background $R i$ became a function of height. This variation demonstrates that a convectively unstable layer was bounded 
above and below by statically stable layers that served to concentrate the turbulent energy into a vertical layer slightly larger than that occupied by a GE Wind 1.5S turbine.

\subsubsection{Potential Turbine Impacts}

The ART turbine operating in the environment described by this case study would have been subjected to inflow conditions that would produced significant aeroelastic and structural responses for a period of at least 3 hours, as demonstrated by Figures 6-20 and 6-33. The sequence of events and probable turbine response seen here is very similar to what we observed during the day-night transition in the San Gorgonio Pass wind farm in California. There the peak fatigue damage in the rotor blades occurred between 22 and $23 \mathrm{~h}$ local standard time. After $21 \mathrm{~h}$, the mean vertical shear exponent decreased to near 0.2 and the dynamic shears seen over a single rotor revolution increased. The probability distributions of the wind speed differences that a 70.5 -m rotor would experience, top to bottom, over one revolution $(\sim 3 \mathrm{~s})$ are presented in Figure 6-34. While the mean difference is the order of $4 \mathrm{~m} / \mathrm{s}$ after $21 \mathrm{~h}$, the range of differences across the rotor would have been low as -2 to a high of $13 \mathrm{~m} / \mathrm{s}$, with the largest encountered difference taking place after 22:20 $\mathrm{h}$ when the rotor layer was the most turbulent.

\subsection{June 23, 2002 - AM Case}

This last case study involves the turbulence associated with the formation of what appeared to be long-period internal gravity waves associated with a decaying LLJ. Such activity is a common occurrence in the nocturnal boundary layer and can be a source bursts of coherent turbulence that last only a few minutes.

\subsubsection{LLJ Morphology}

The period of interest for the case study was 2:10 to 4:20 h LST on June 23, 2002. The vector wind profile derived from the Scintec SODAR is shown in Figure 6-35. While the entire period between midnight and $06 \mathrm{~h}$ is presented, we will only analyze the segment indicated by the black box in the center (i.e., from 2:10 to 4:20 h). The LLJ reached its peak velocity about 2:30 h, and the marked rotation of the wind direction from the south through west indicates that an inertial oscillation was likely responsible.

Details of the wind flow up to a height of $450 \mathrm{~m}$ are shown as contours of SODAR-derived wind speed and direction (Figures 6-36 and 6-37, respectively). The LLJ maximum is located at about $250 \mathrm{~m}$. A close examination of the wind speed profile below $125 \mathrm{~m}$ in Figure 6-36 reveals a wave-like pattern beginning near $03: 10 \mathrm{~h}$ and extending to the end of the period at 4:20 $\mathrm{h}$. The time histories of the 5-minute mean wind speeds from the cup anemometers installed at 3-, 52-, and 113-m elevations are each plotted against its own ordinate axis (Figure 6-38). This graph reveals a growing wave motion that extends all the way to the ground $(3 \mathrm{~m})$ and which reaches its peak amplitude at approximately 3:50 h. The period of the wave is approximately 30 minutes. It is likely to have been an internal shear-generated buoyancy or gravity wave that was triggered by the dissipating LLJ. Waves such as these can destabilize the flow locally as they propagate through. They are a common feature of the nocturnal boundary layer. 


\subsubsection{Vertical Shear Characteristics}

We have again subdivided the study period into three segments. The first ranges from 2:10 to $2: 50 \mathrm{~h}$ and includes the early formation phase of the wave motion. The second encompasses the period when the wave has reached is peak between 03 to 3:50 h. The last segment from 04 to 4:20 $\mathrm{h}$ corresponds to the wave decay. The SODAR-derived wind profiles for each of segments is presented in Figure 6-39. During the early stages of the jet dissipation the vertical shear below the jet maximum was relatively intense and steady below $200 \mathrm{~m}$. The period when the wave amplitude was at its peak saw a reduction in the overall wind speed, but the shear intensity remains about the same. Finally, as the wave begins to decay in the last segment there is a further reduction in wind speed, but again the shear characteristics do not change much below $200 \mathrm{~m}$. The variation of the shear exponent presented as contours are plotted in Figure 6-40. While the bulk of the high shear values remain above the top of the GE $1.5 \mathrm{~S}$ rotor $(115 \mathrm{~m})$, high values associated with the large wave amplitude centered near $3: 20 \mathrm{~h}$ extend to the bottom of the rotor. This is a common occurrence when gravity waves are active (i.e., they periodically increase and then relax the shear as they propagate). Also again, future turbines with rotors extending up to $200 \mathrm{~m}$ would experience significant shears, as is indicated in Figure 6-40.

\subsubsection{Turbulence and Potential Turbine Impacts}

The time series of background turbulence scaling parameters for this case are plotted in Figure 641. Immediately noticeable in Figure 6-41a is that the rotor layer $(52-113 \mathrm{~m}) R i$ value remains at or above +0.1 (the weakly stable upper boundary) before becoming very stable by exceeding the $R i_{c}$ upper limit at 4:20 h. Figure 6-41b shows that the ART turbine generally would not have responded to the wave-induced inflow (peak $E_{c o h}<4 \mathrm{~m}^{2} / \mathrm{s}^{2}$ ), except at $3: 40 \mathrm{~h}$. The period associated with the largest wave amplitude is indicated with a light crosshatch where a peak in $\sigma_{w}$ also occurs. Here also the $l_{b} / D$ ratio reaches its peak, signifying that the characteristic scale of waves was approaching half the size of the rotor disk. The 10-minute mean vertical fluxes of turbulent kinetic energy $\left(\overline{w^{\prime} E}\right)$ and coherent turbulent kinetic energy $\left(\overline{w^{\prime} E_{c o h}}\right)$ and the vertical velocity $\left(w^{\prime}\right)$ for this study period are shown in Figure 6-42. A comparison of the nature of the peaks in Figure 6-38 with Figure 6-42 shows that bursts of turbulent energy are associated with both positively increasing horizontal and vertical winds. While more information is needed, it appears that the wave may have broken during the $3: 50 \mathrm{~h}$ period, as indicated by the sudden negative vertical velocity and downward fluxes of $E$ and $E_{c o h}$. Though not particularly strong in this case, this sequence demonstrates how the lifecycle of internal gravity waves may affect wind turbine response dynamics. The overall response of the ART turbine rotor, if exposed to these conditions as quantified by the vertical variation of peak $E_{c o h}$, is shown in Figure 6-43. Only the levels of peak $E_{c o h}$ during the period of 3:40 to $3: 50 \mathrm{~h}$ would have been sufficient to induce a measurable response.

The variation in dynamic shear (over a single GE rotor revolution) is presented in Figure 6-44. The probability density distributions have been grouped to correspond to the periods associated with each of the wave peaks. With the exception of the last one, the distributions included in each group are similar, indicating that comparable processes are likely taking place. The broadening of the distributions at 04 and 04:10 $\mathrm{h}$ in the last group reflect the decaying nature of the wave structure. The rapid decrease and extreme narrowing of the distribution at $4: 20 \mathrm{~h}$ is in response to the equivalently rapid increase in stability shown in Figure 6-41a. 


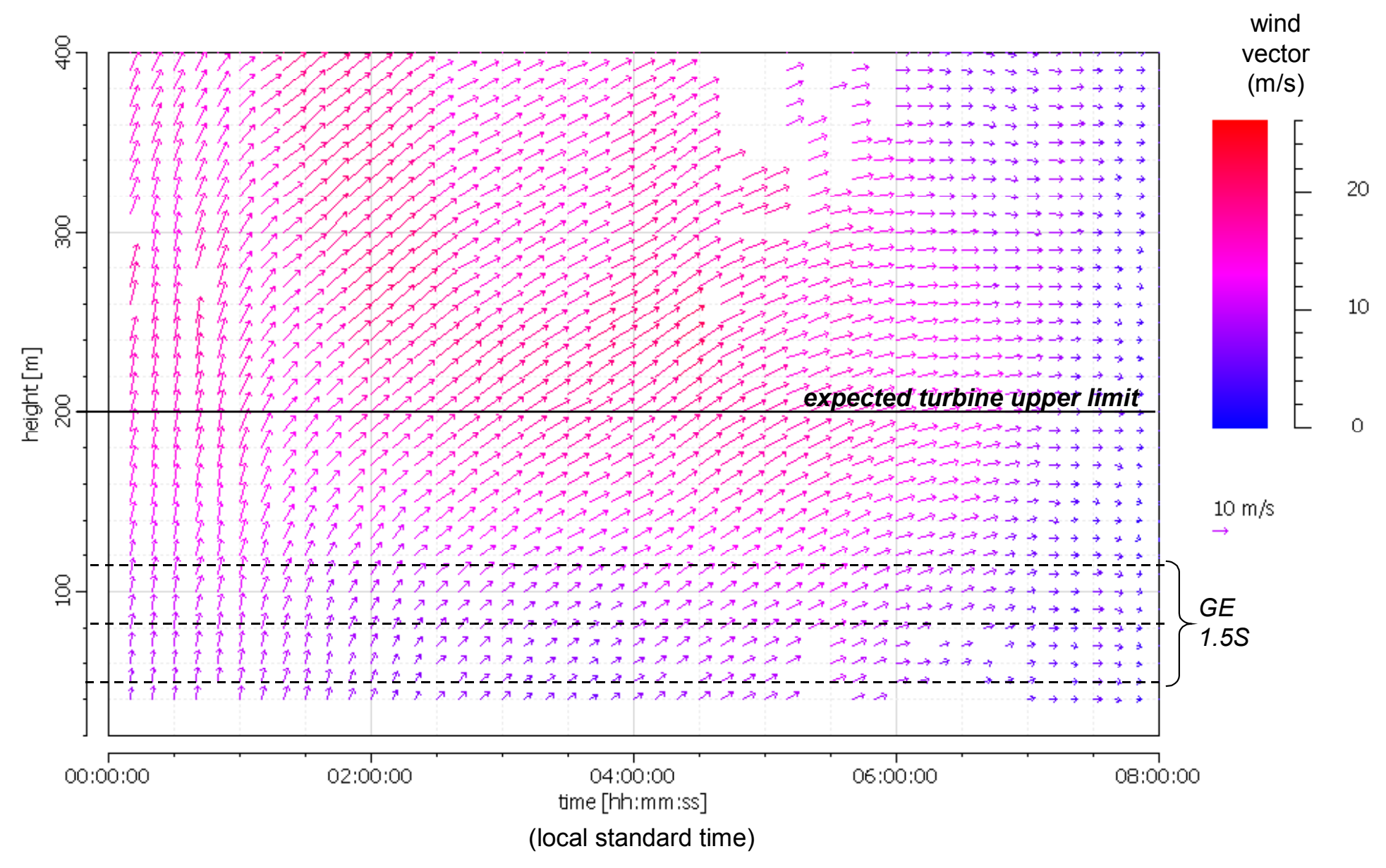

Figure 6-1. SODAR-derived 10-minute mean wind vector profiles for 00 to $08 \mathrm{~h}$ LST on 17 June 2002 


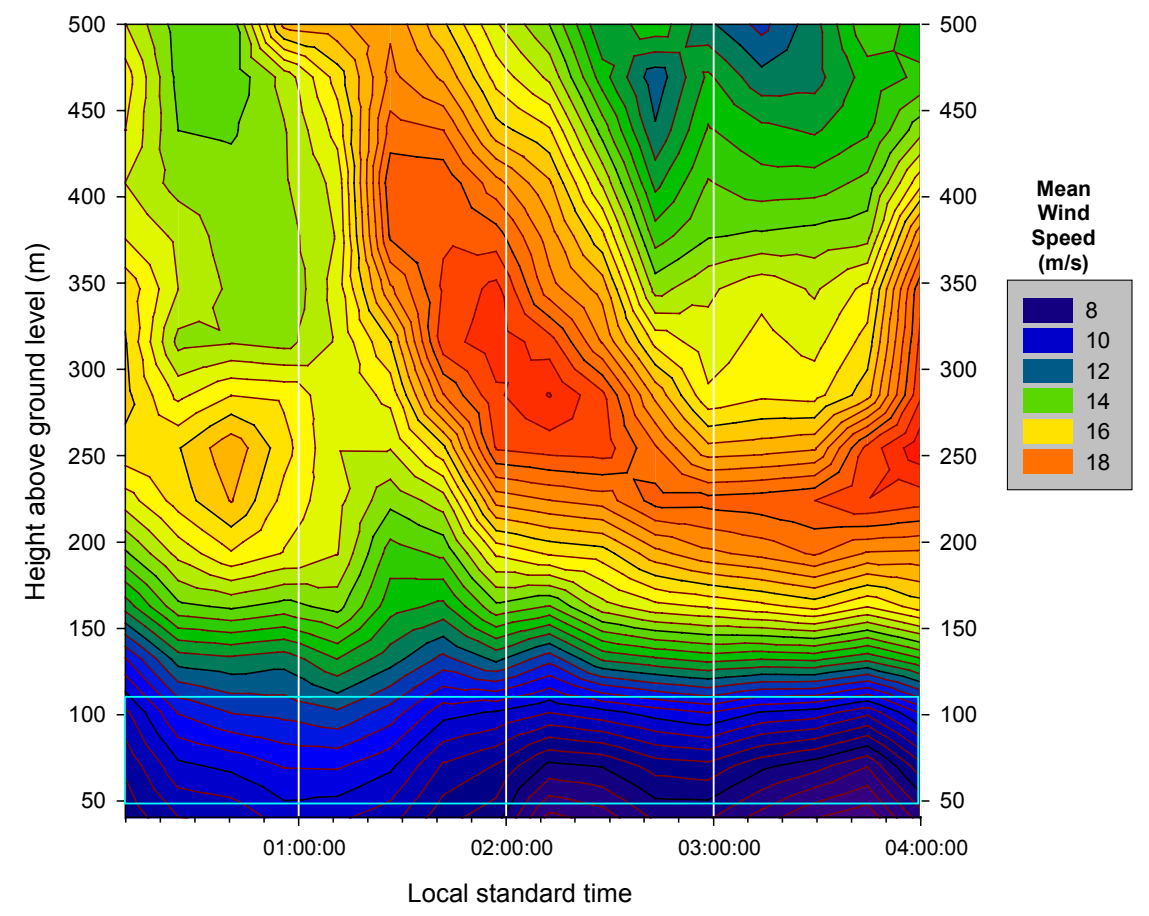

Figure 6-2. SODAR-derived mean wind speed profile contours for 17 June 2002 - AM Case

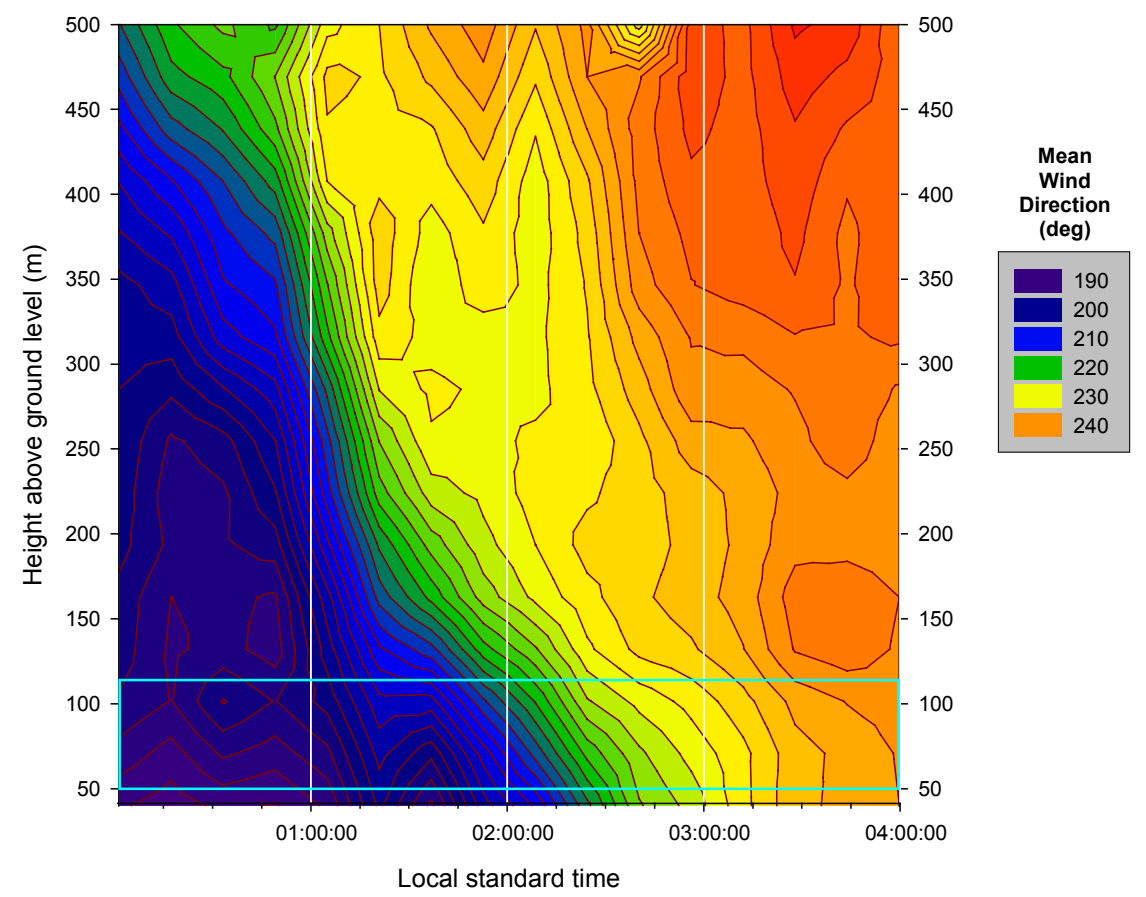

Figure 6-3. SODAR-derived mean wind direction profile contours for 17 June 2002 - AM Case 

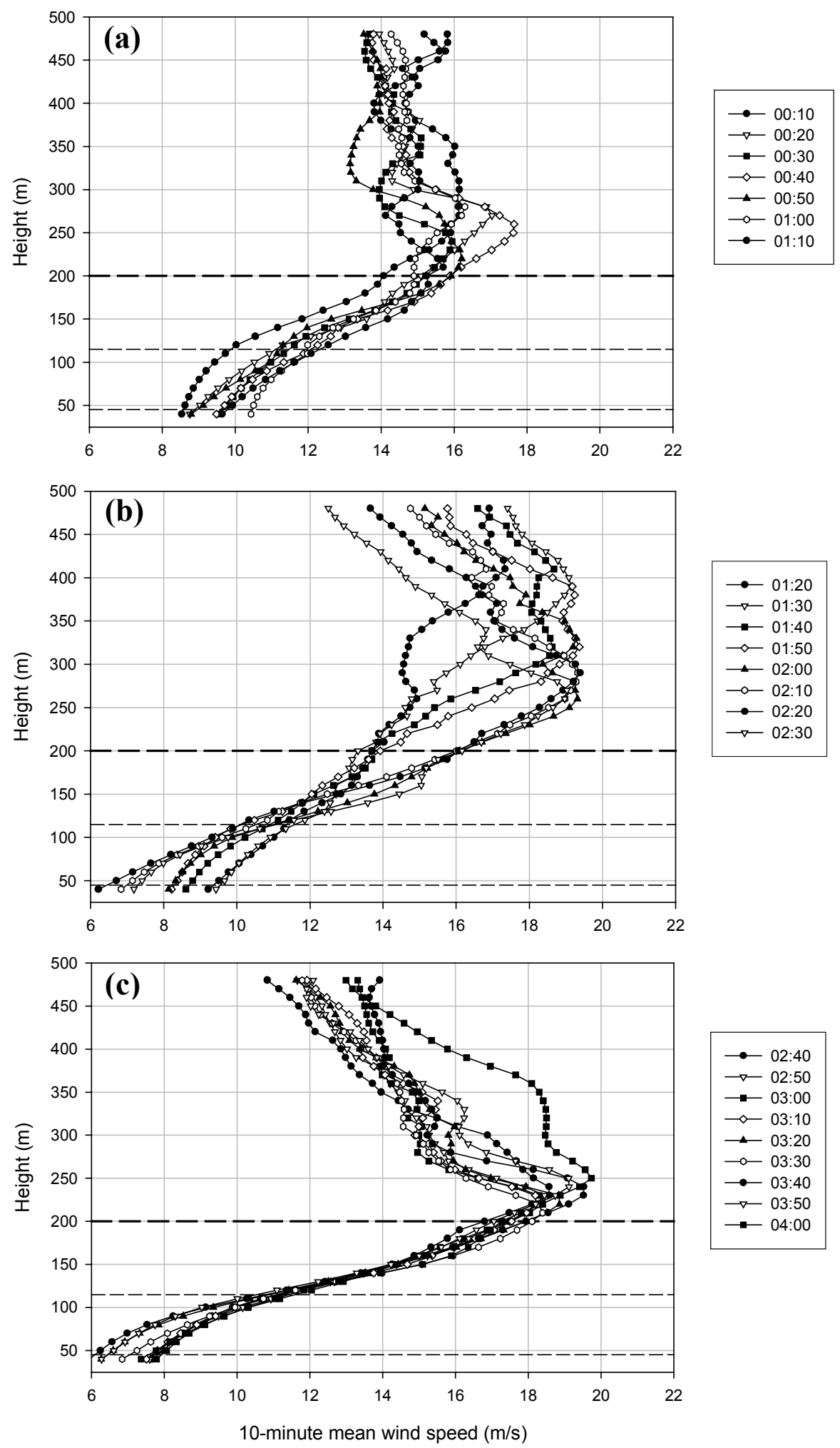

Figure 6-4. SODAR-derived wind speed profiles for 17 June 2002 - AM Case 


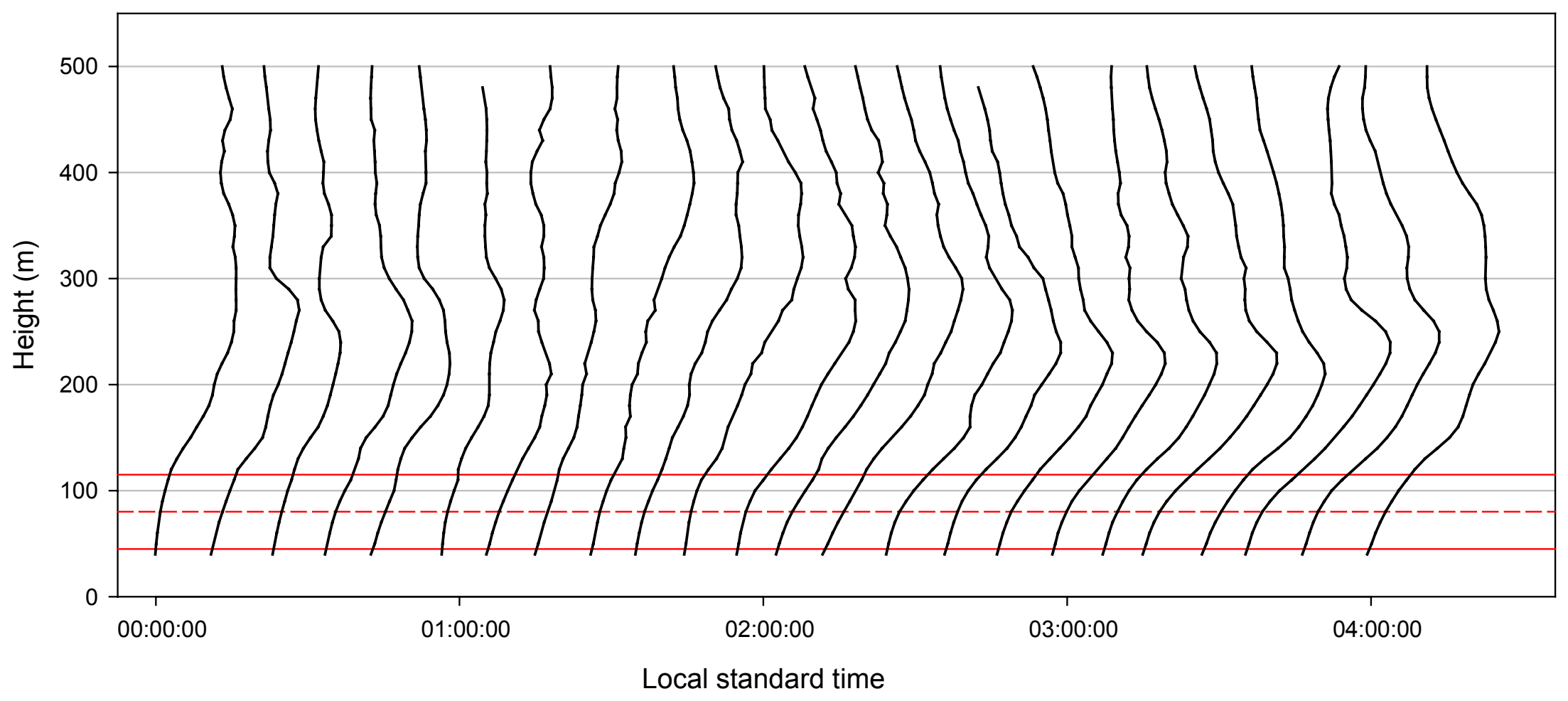

Figure 6-5. SODAR-derived wind profile evolution for 17 June 2002 - AM Case 

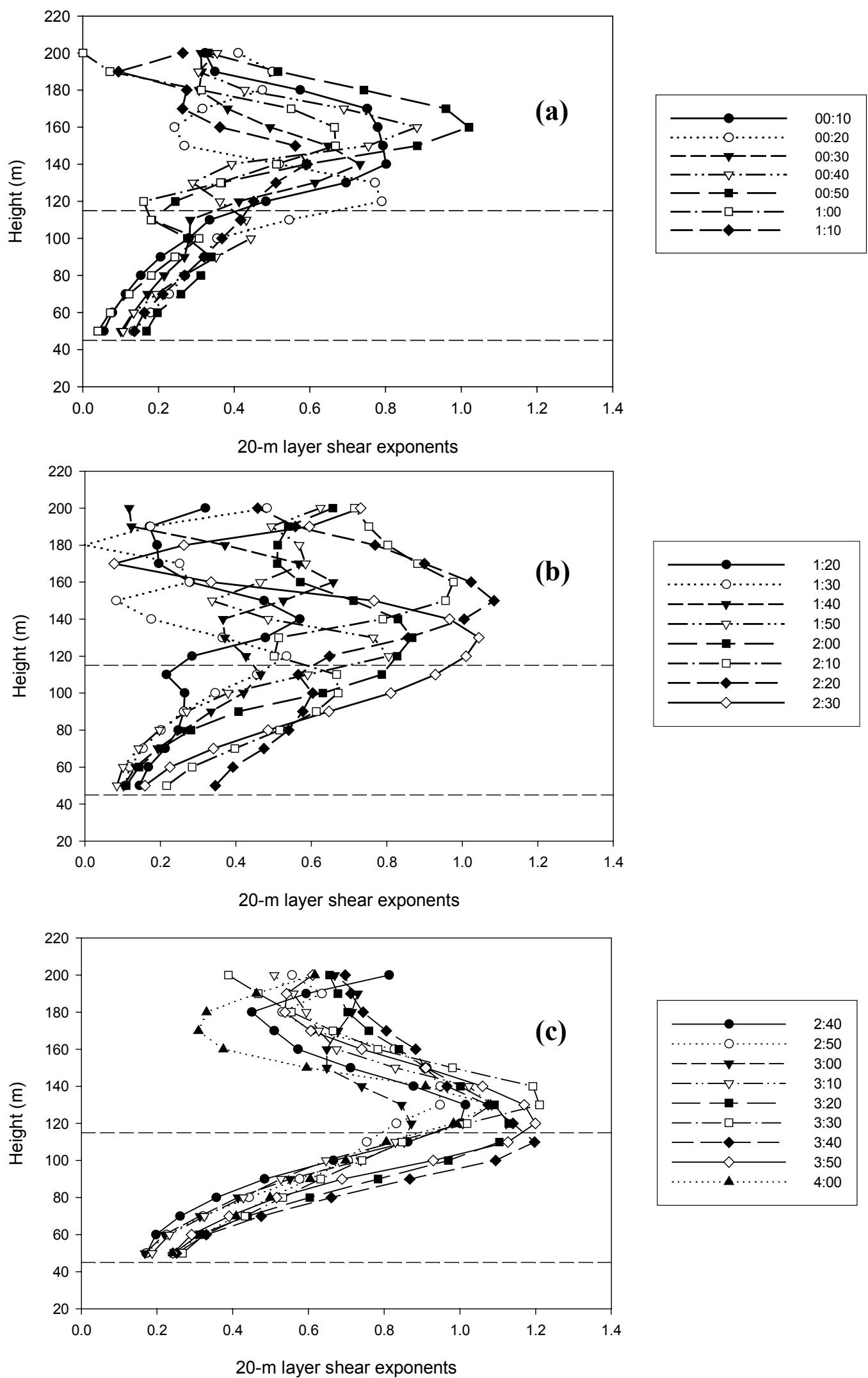

Figure 6-6. SODAR-derived wind shear profiles for 17 June 2002 - AM Case 


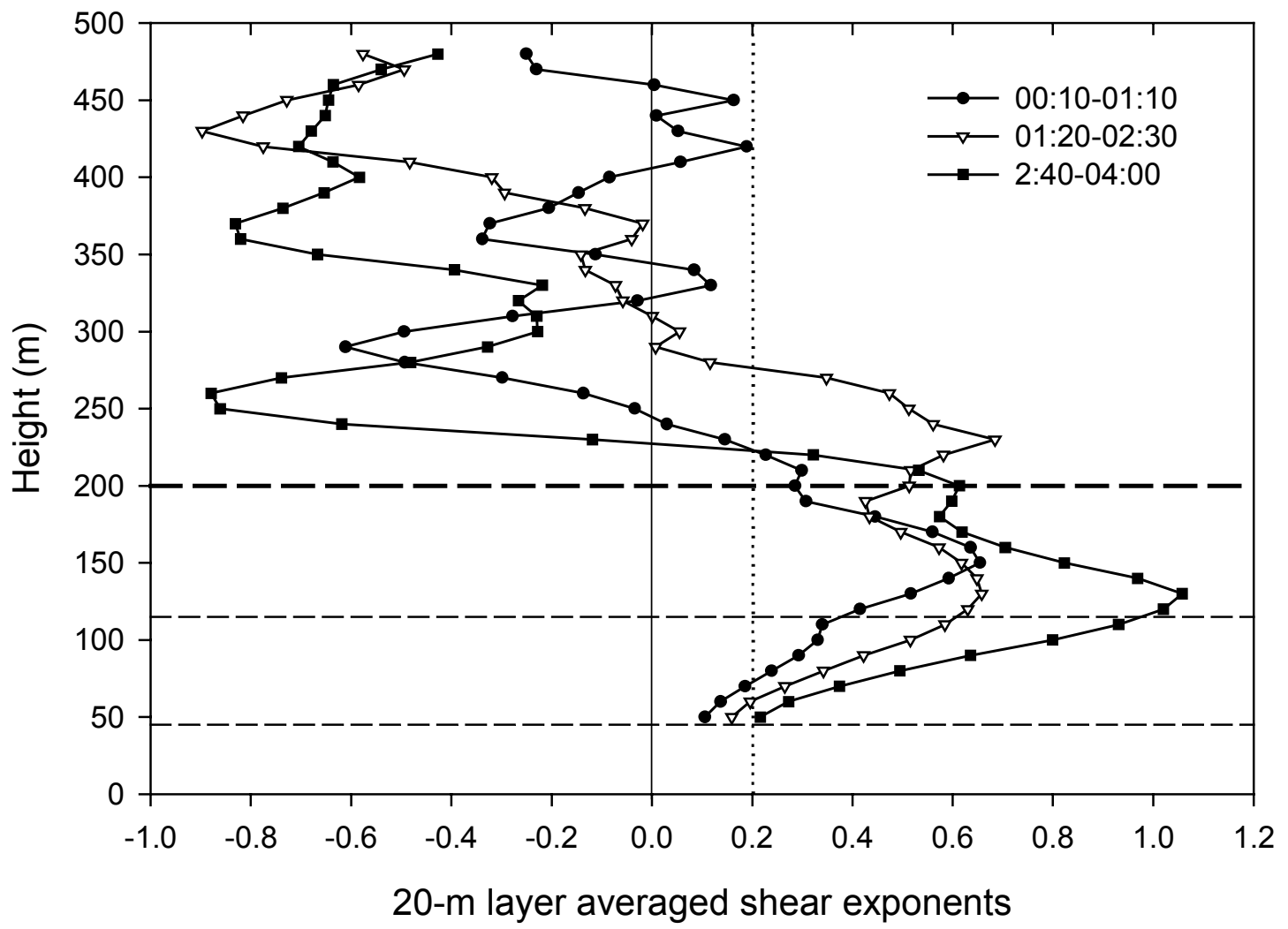

Figure 6-7. Sodar-derived averaged wind shear profiles for 17 June 2002 - AM Case 


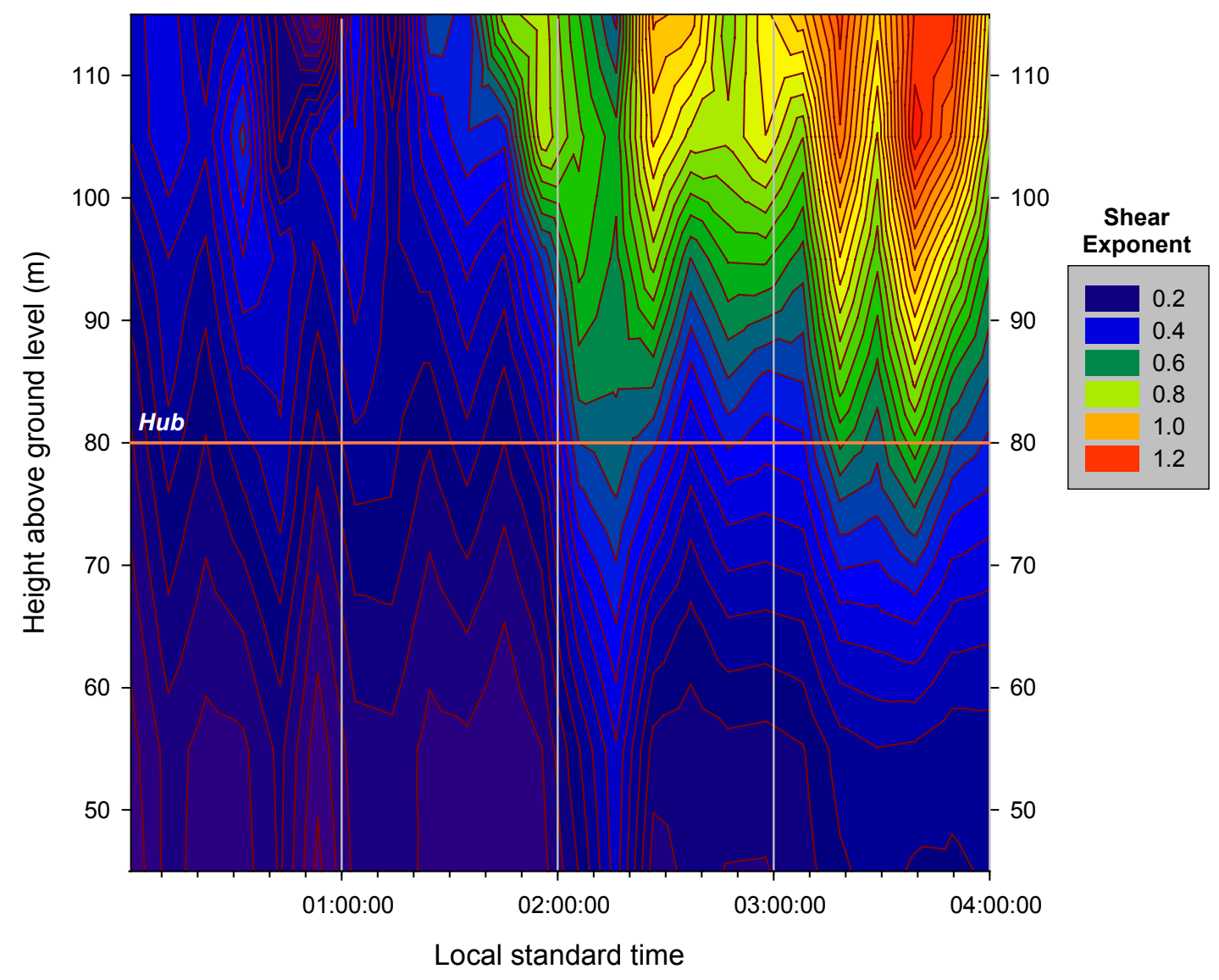

Figure 6-8. SODAR-derived vertical shear profile contours for 17 June 2002- AM Case 

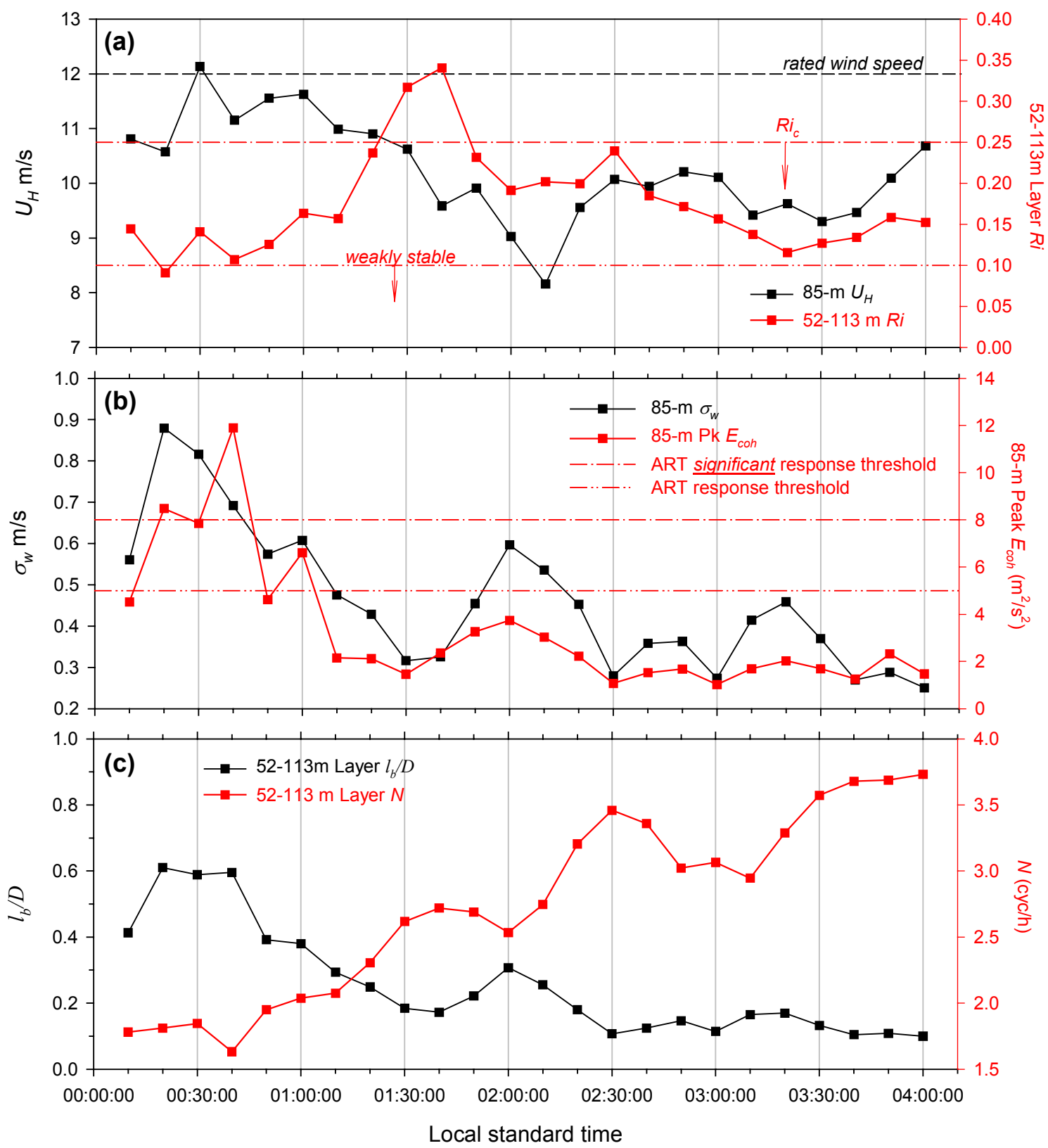

Figure 6-9. Time histories of 10-minute mean inflow parameters for 17 June 2002 - AM Case 


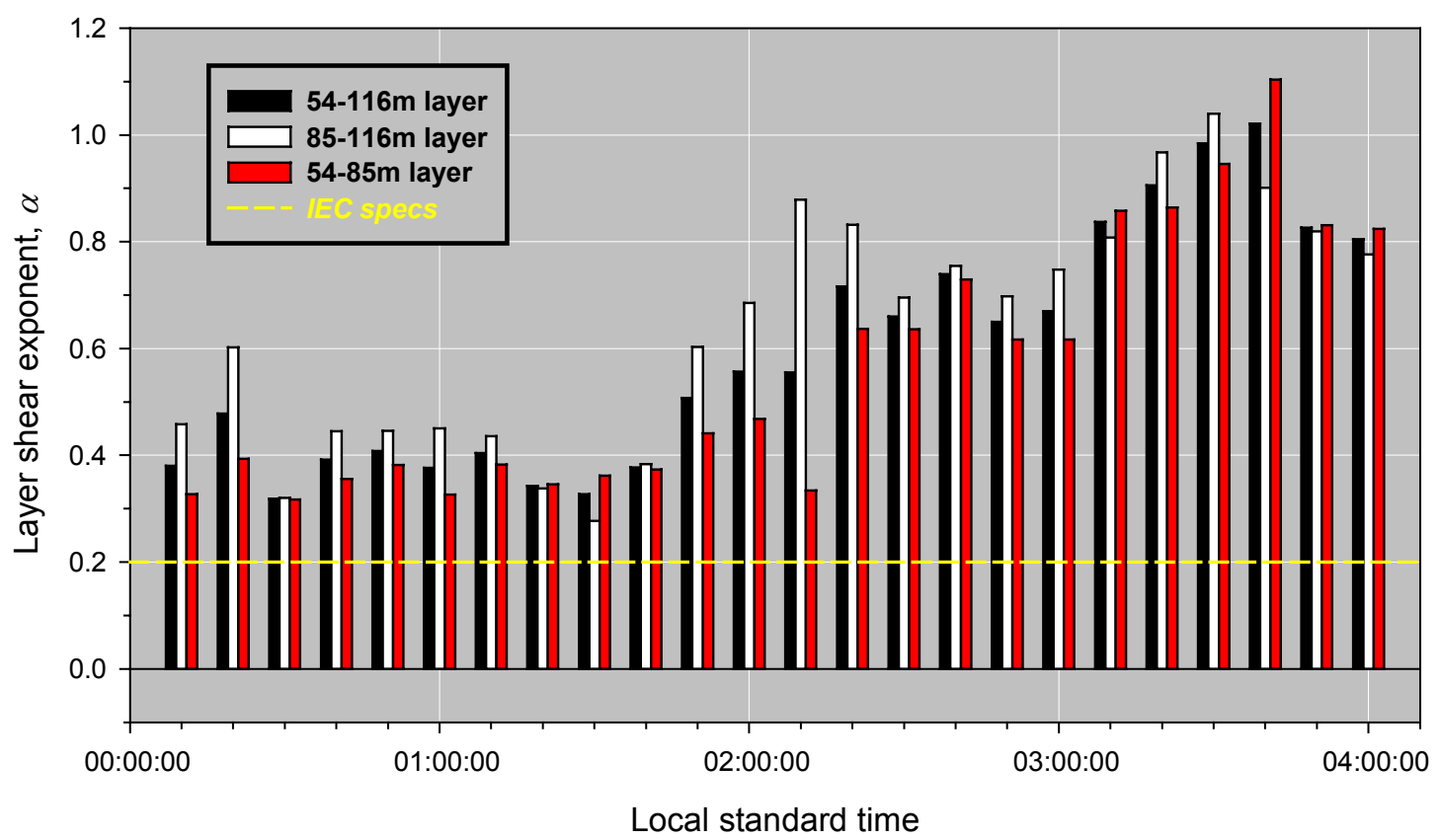

Figure 6-10. Histogram of 10-minute layer mean shear exponents for 17 June 2002 - AM Case

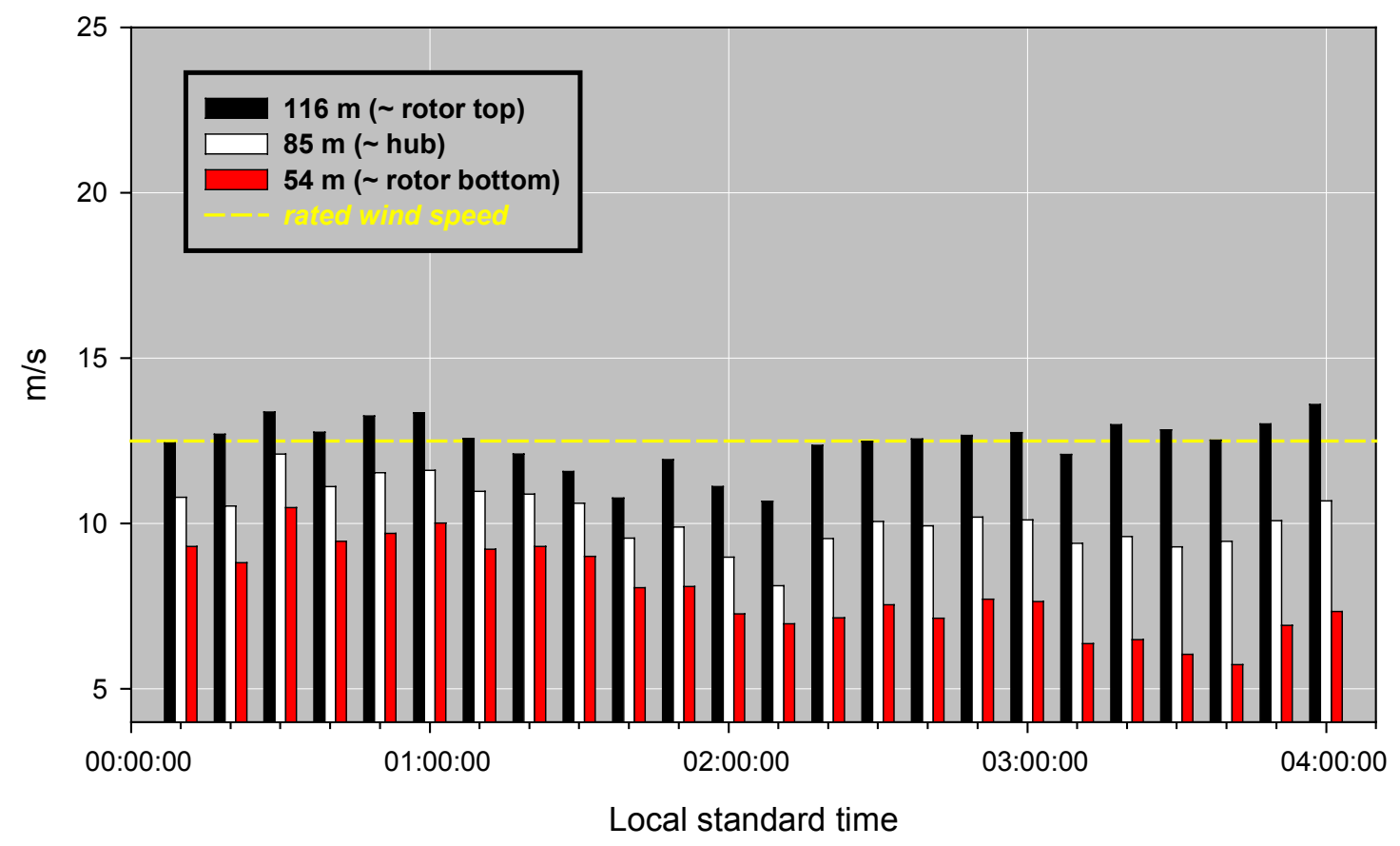

Figure 6-11. Histogram of 10-minute mean wind speeds for 17 June 2002 - AM Case 


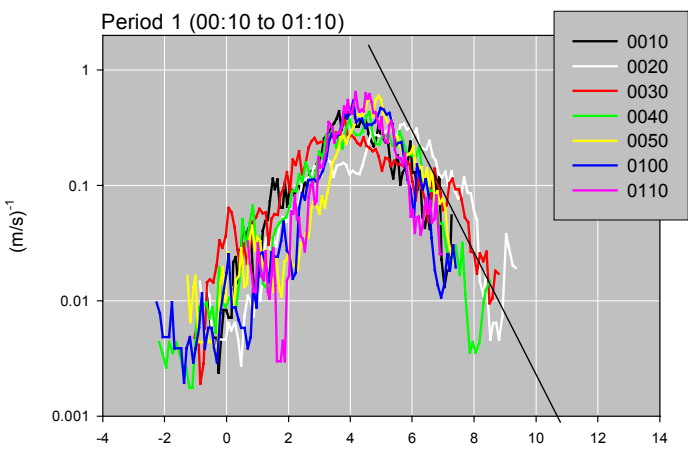

$\mathrm{m} / \mathrm{s}$

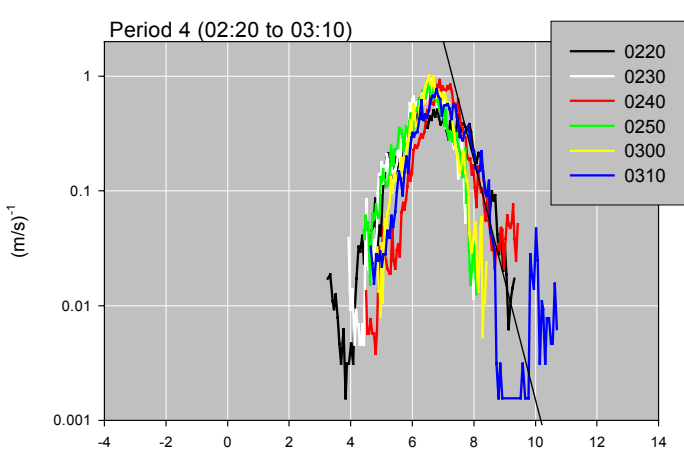

$\mathrm{m} / \mathrm{s}$

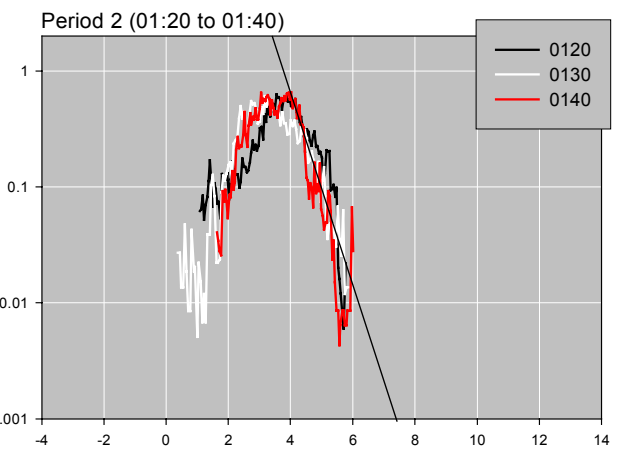

$\mathrm{m} / \mathrm{s}$

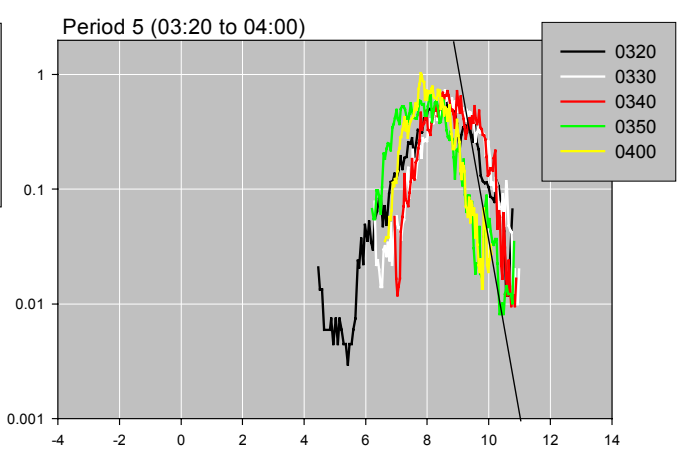

$\mathrm{m} / \mathrm{s}$

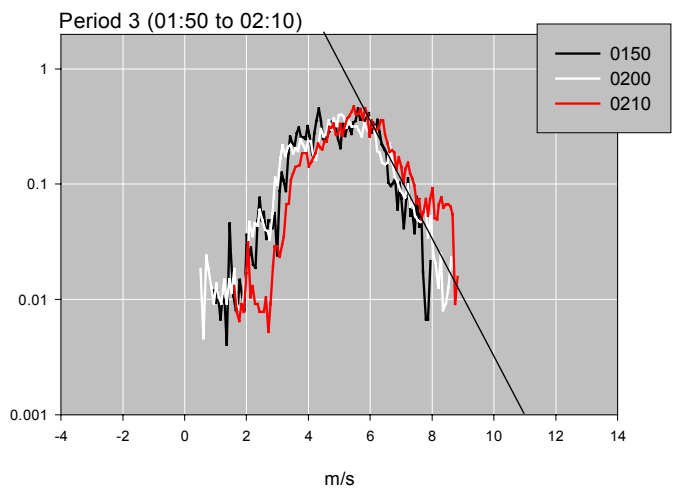

17 June 2002 AM Case

3-Sec Wind Speed Difference

Probability Density Distributions

116-54 m Height Range (Full Rotor)

(Scaled for $70.5 \mathrm{~m}$ rotor diameter)

Figure 6-12. Probability density distributions of 3-s wind speed differences for 17 June 2002 - AM Case 


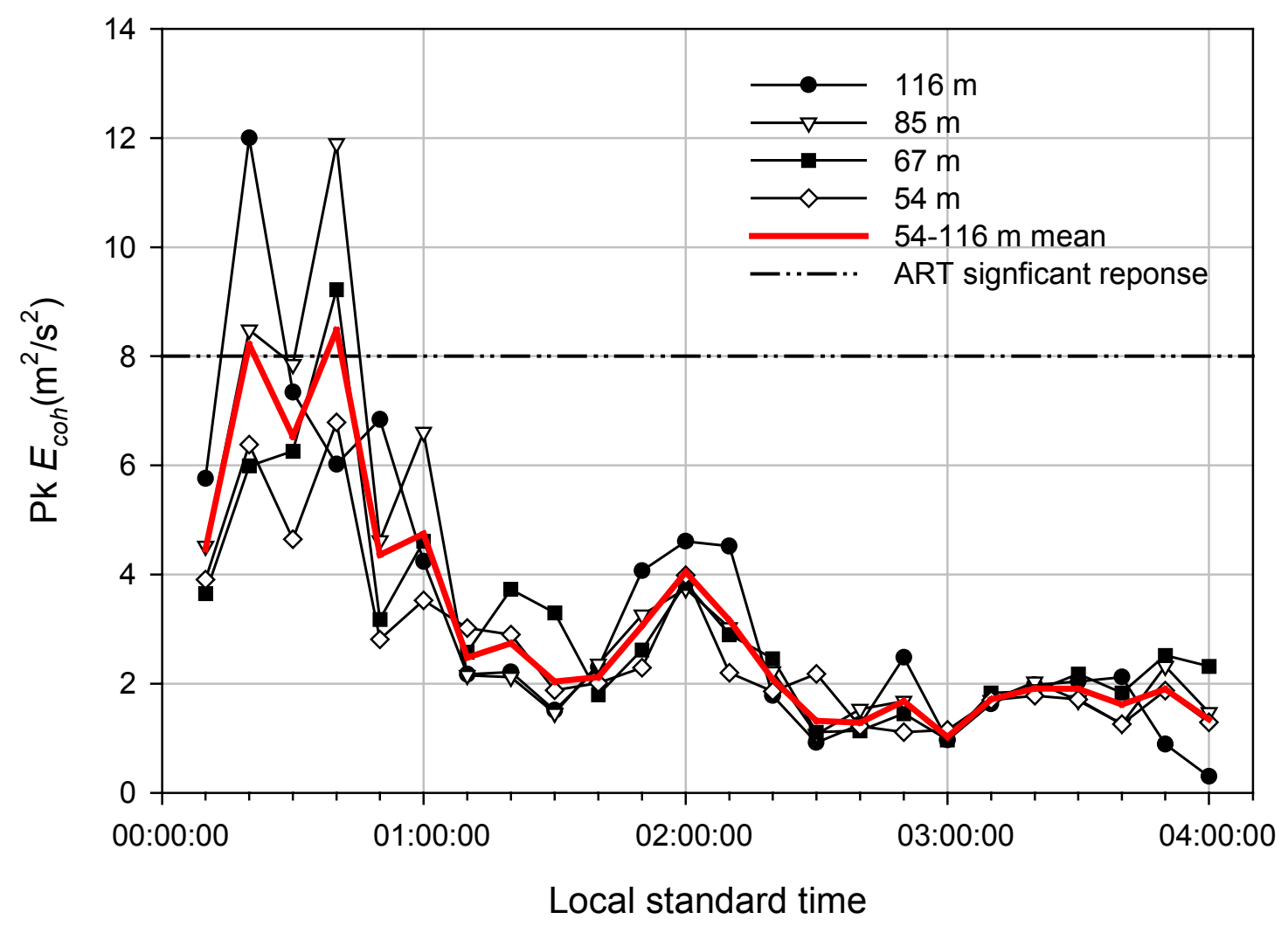

Figure 6-13. Time histories of peak coherent turbulent kinetic energy $E_{c o h}$ by height for 17 June 2002 - AM Case 


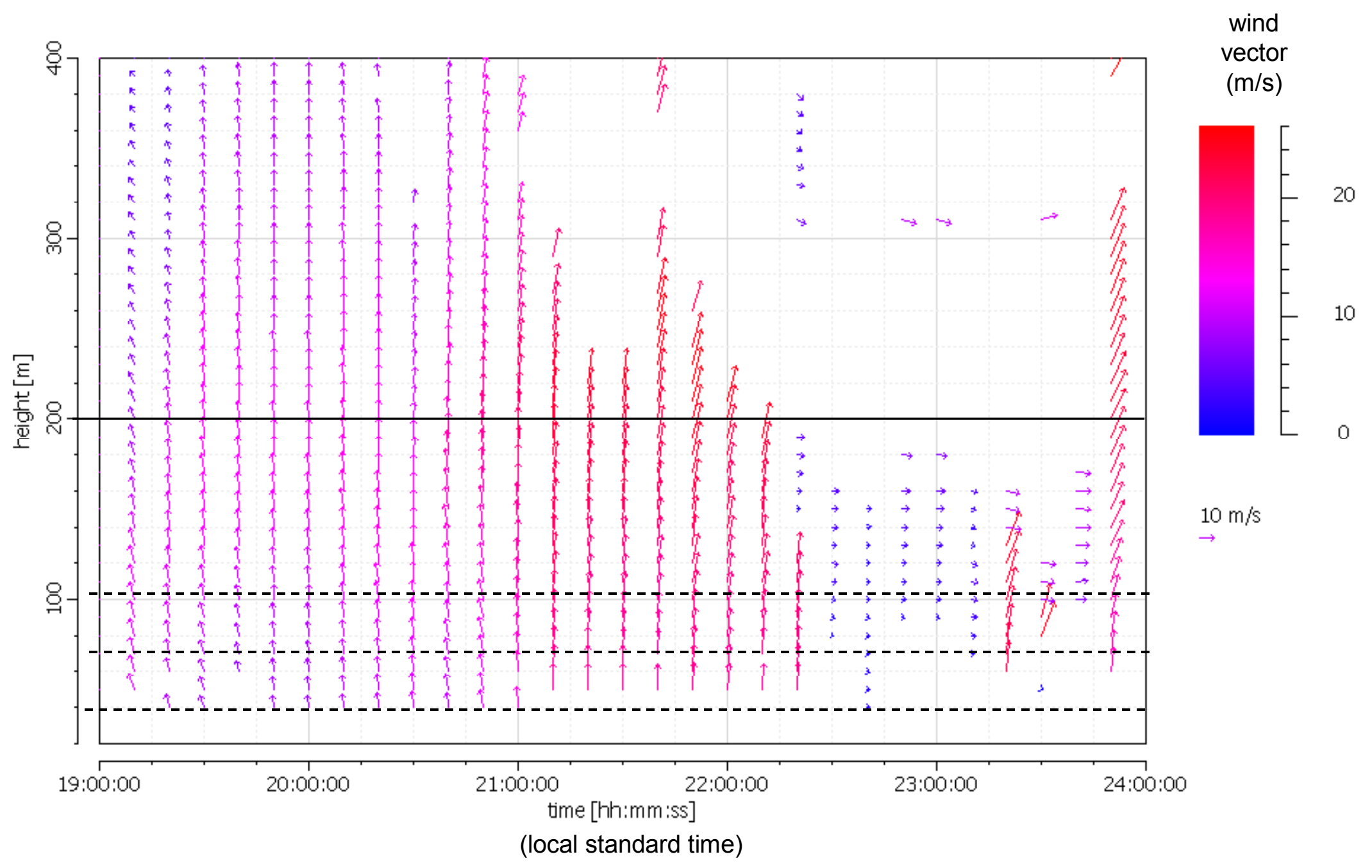

Figure 6-14. SODAR-derived 10-minute mean wind vector profiles for 19 to $24 \mathrm{~h}$ LST on 17 June 2002 


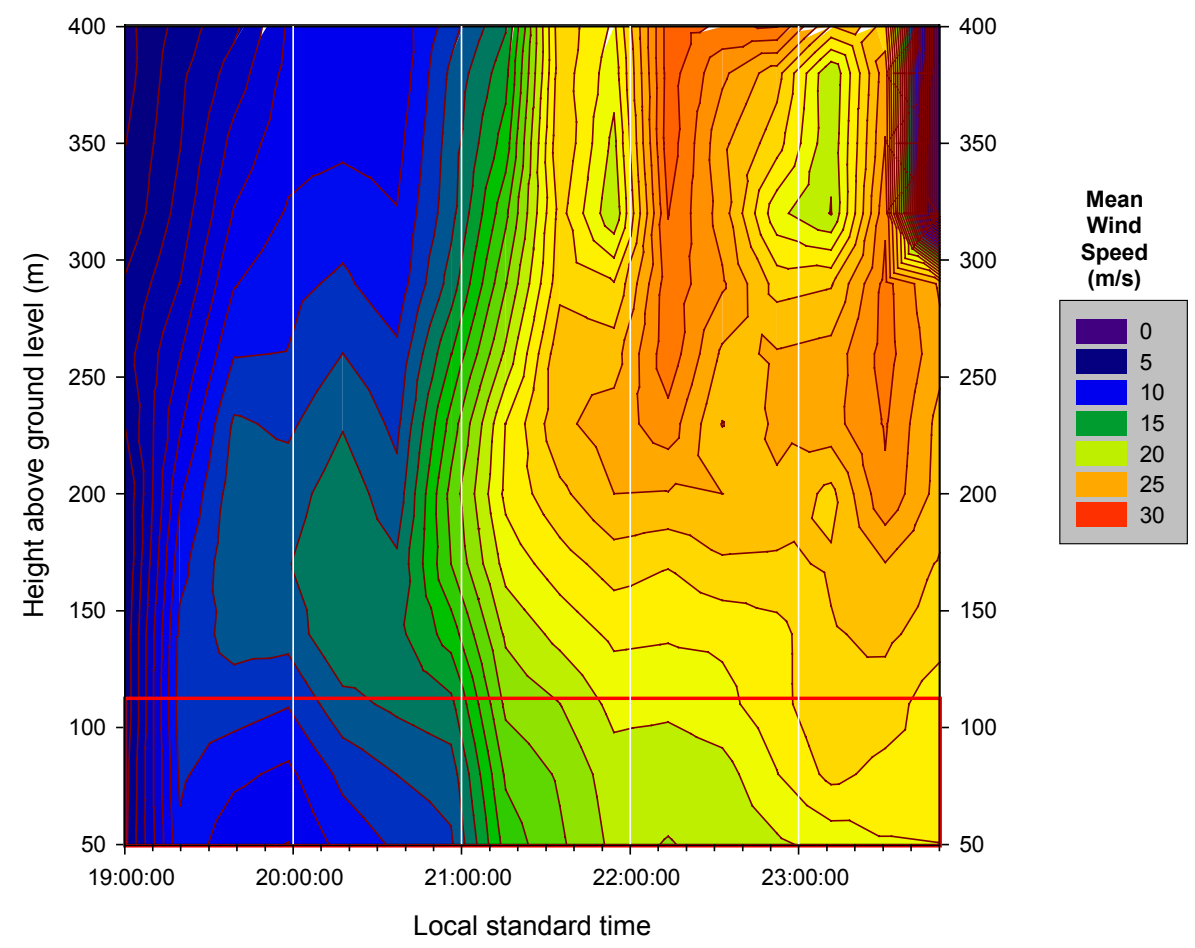

Figure 6-15. SODAR-derived mean wind direction profile contours for 17 June 2002 - PM Case

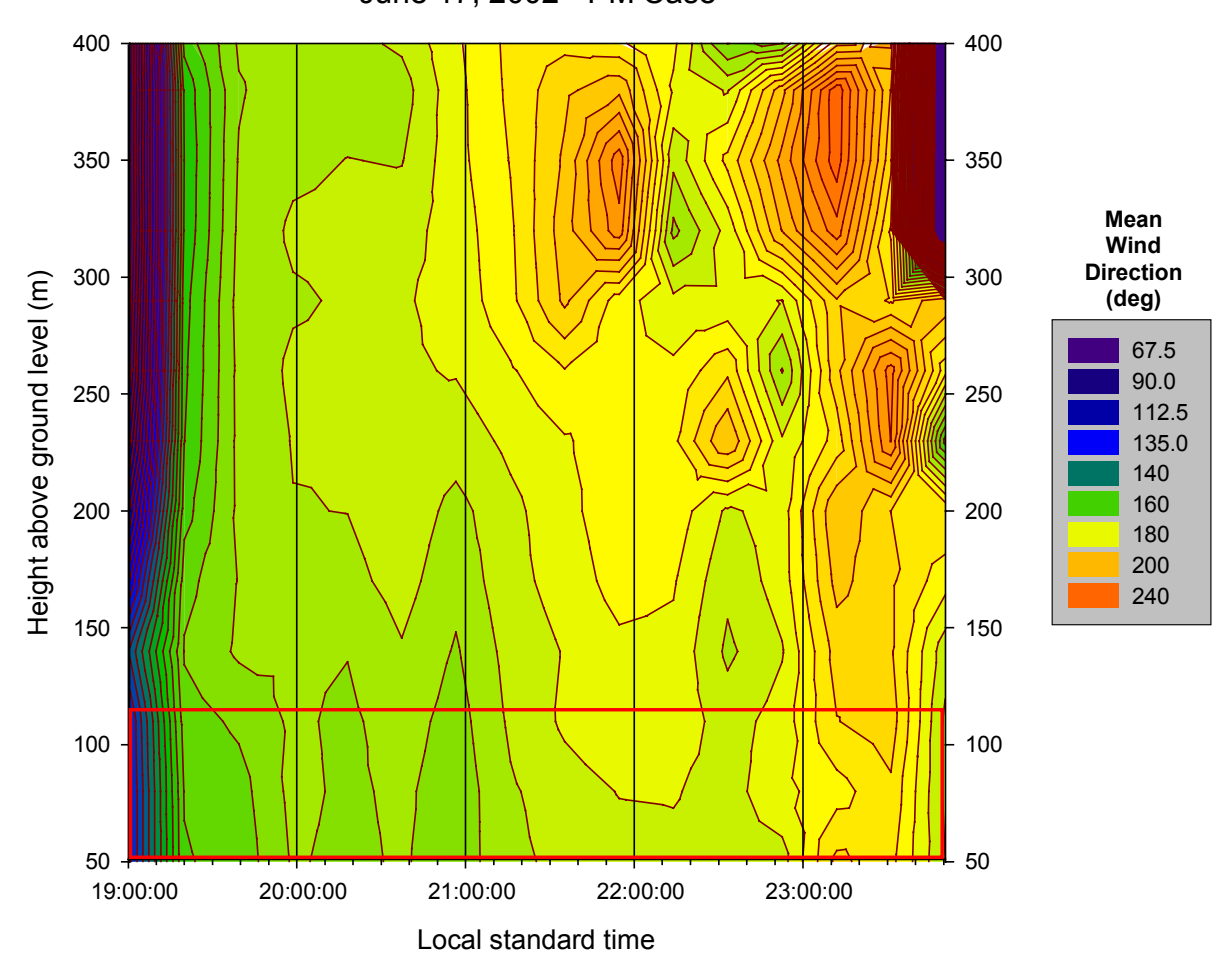

Figure 6-16. SODAR-derived wind speed profiles for 17 June 2002 - PM Case 

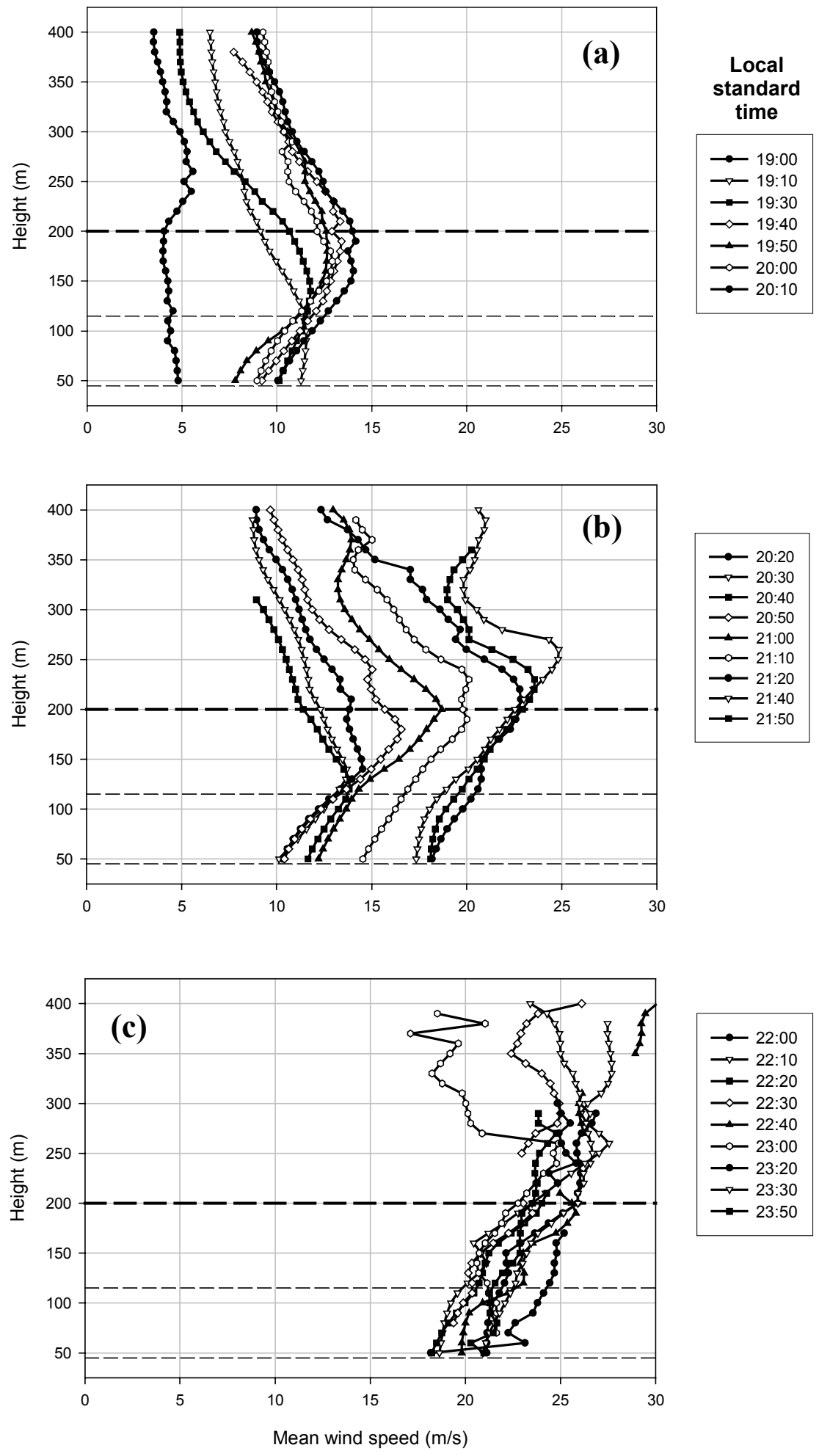

Figure 6-17. SODAR-derived wind speed profiles for 17 June 2002 - PM Case 

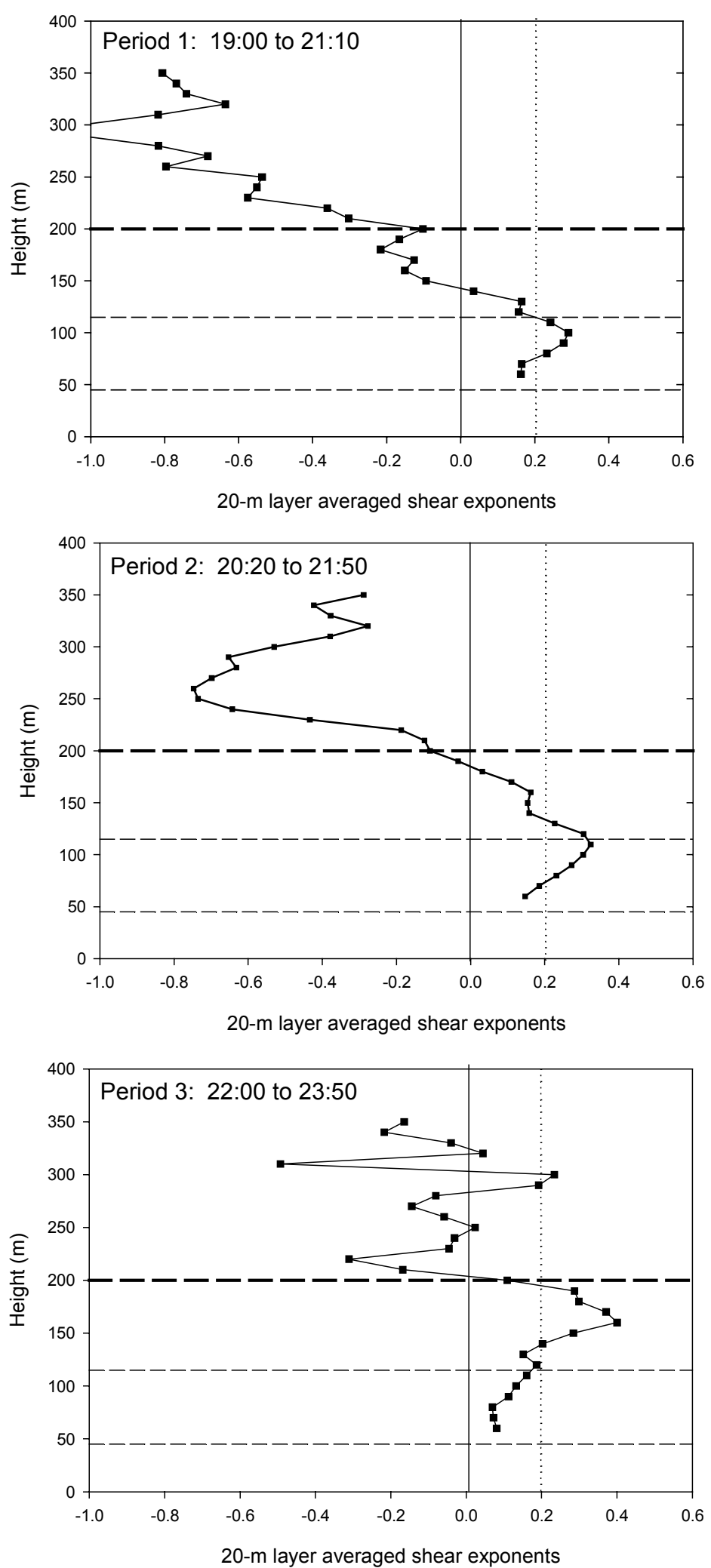

Figure 6-18. SODAR-derived averaged wind shear profiles for 17 June 2002 - PM Case 


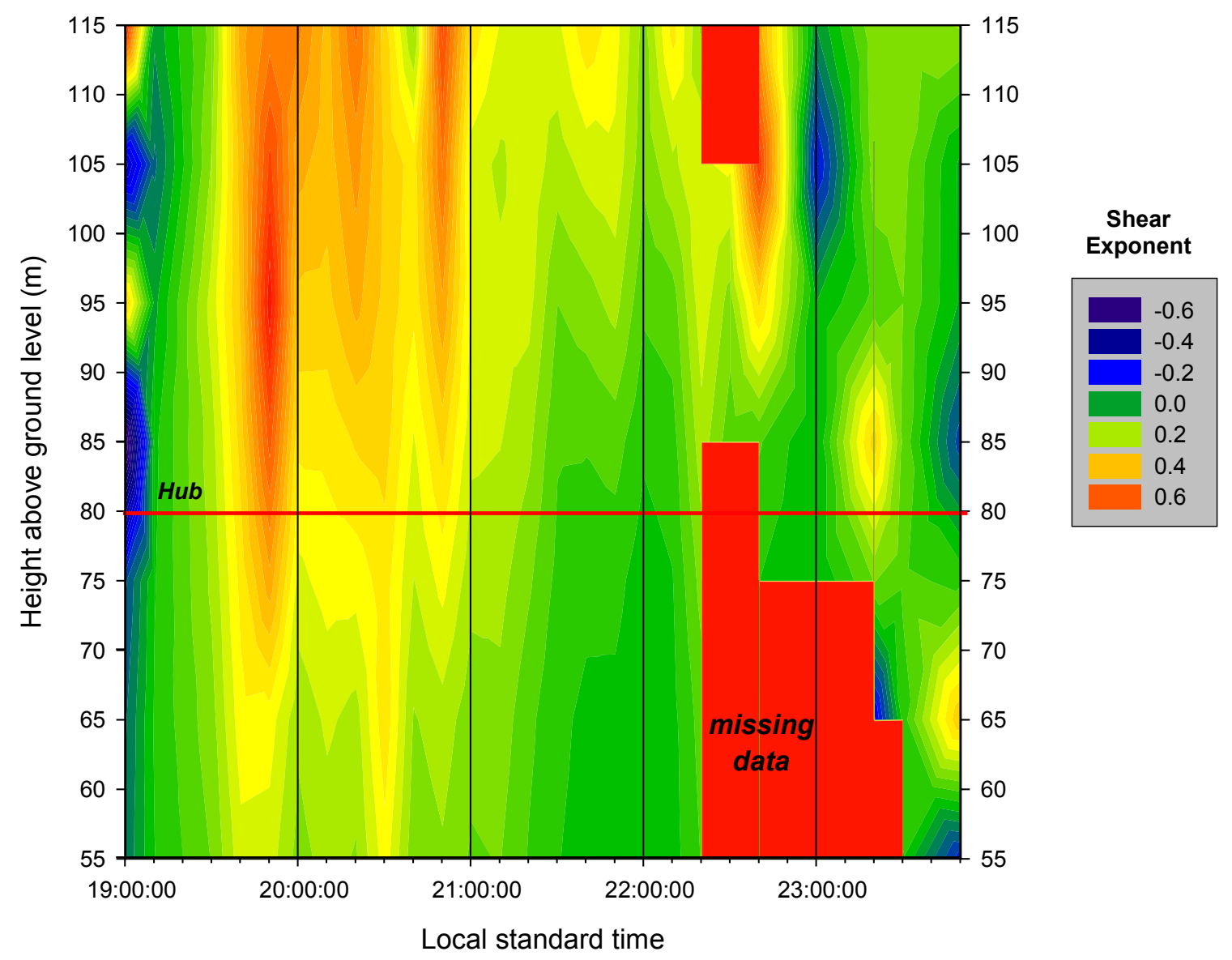

Figure 6-19. SODAR-derived vertical shear profile contours for 17 June 2002 - PM Case 

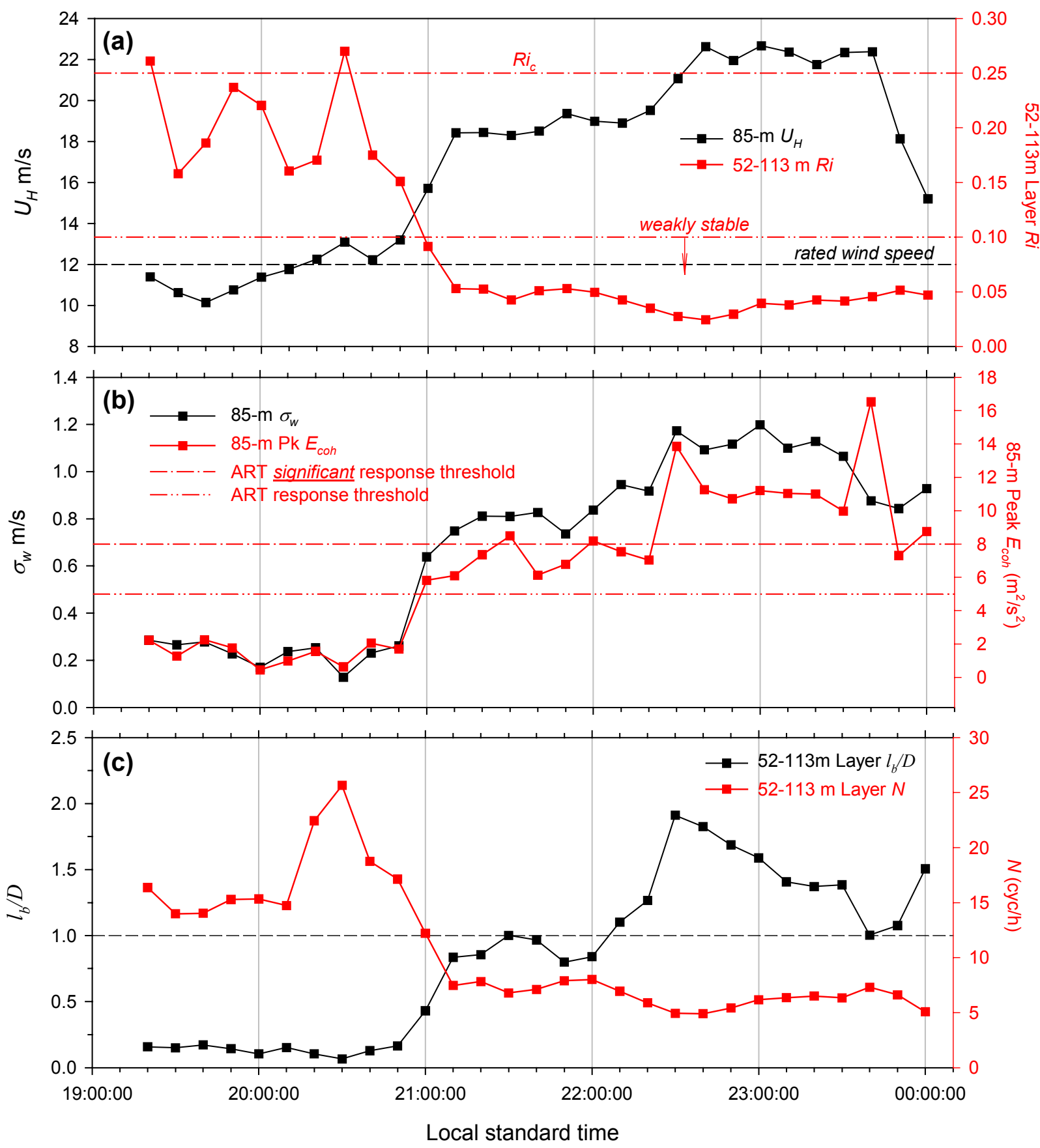

Figure 6-20. Time histories of 10-minute mean inflow parameters for 17 June 2002 - PM Case 
Buoyancy length scale vs Layer mean Peak Coh TKE

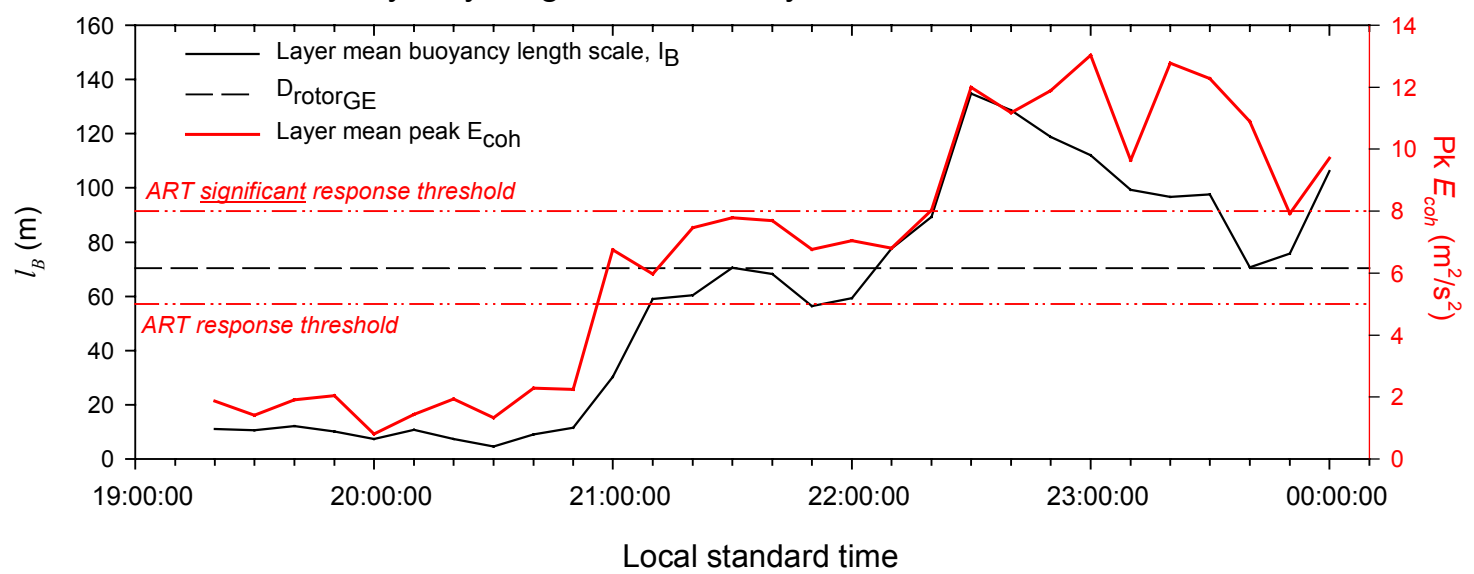

Turbulence intensities vs Layer mean Peak Coh TKE

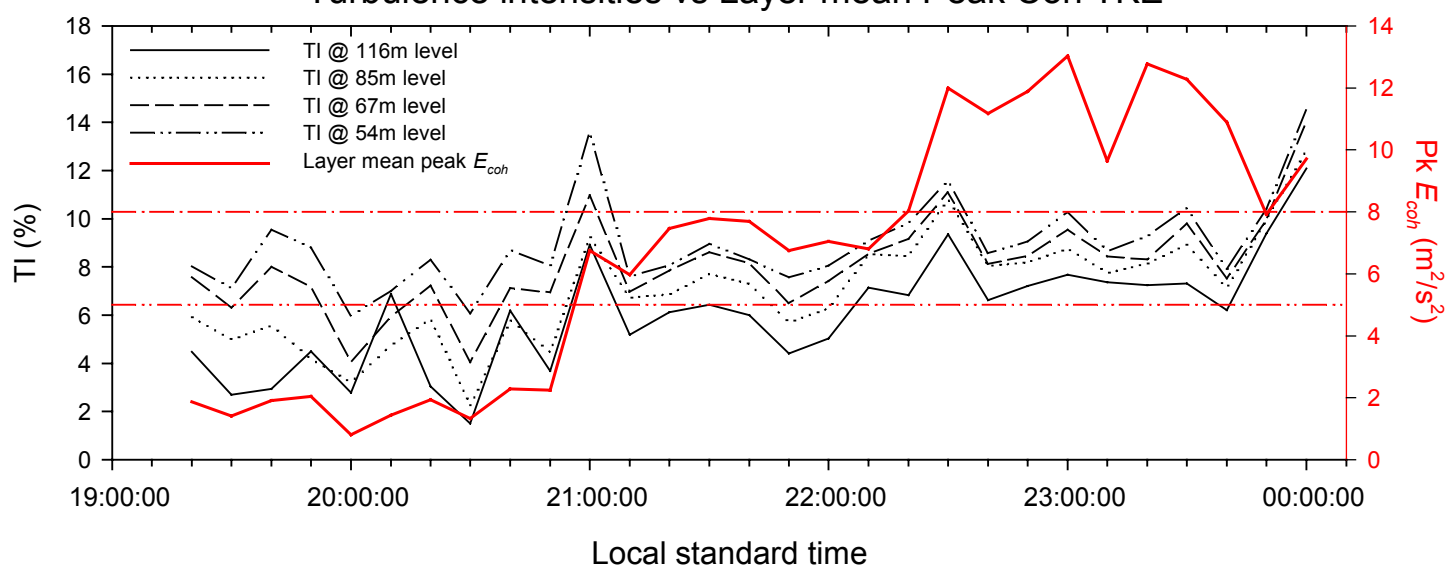

Rotor disk shear exponents vs gradient Richardson number

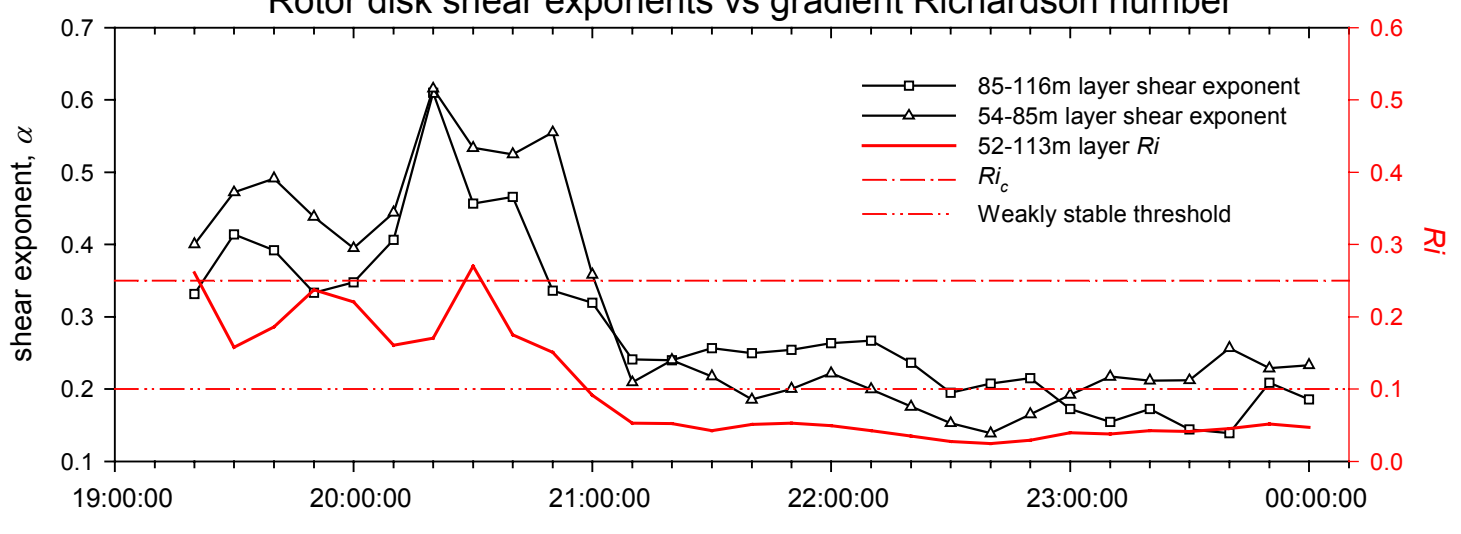

Local standard time

Figure 6-21. Time histories of buoyancy length scale $I_{b}$, peak $E_{c o h}$, turbulence intensities, rotor-disk shear exponents and Ri for 17 June 2002 - PM Case 

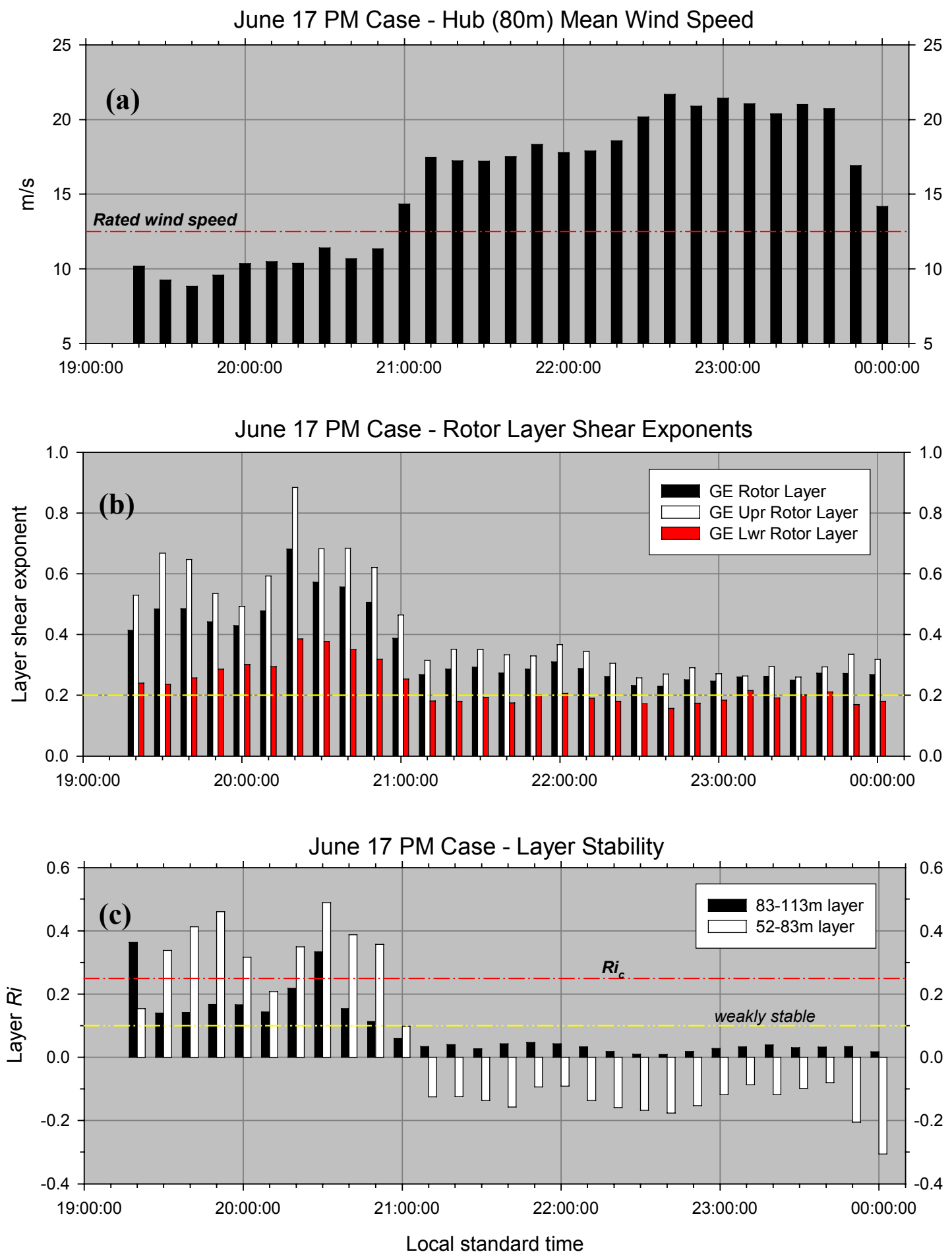

Figure 6-22. Time histories of estimated (a) 80-m mean wind speed, (b) rotor-layer shear exponents, and (c) layer stability for 17 June 2002 - PM Case 


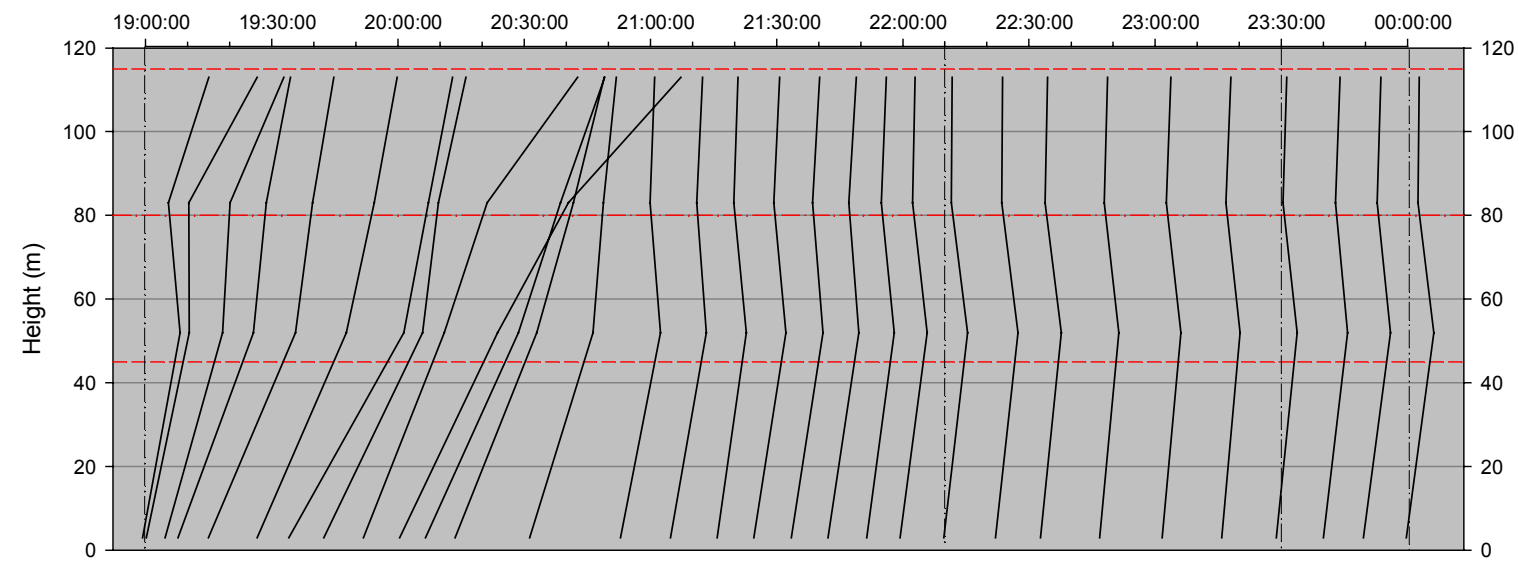

Potential (thermodynamic) temperature $\left({ }^{\circ} \mathrm{K}\right)$

June 17 PM Case - Mean Sonic ${ }^{\#}$ Wind Profiles 19:30 to 00:00 MST

(b)

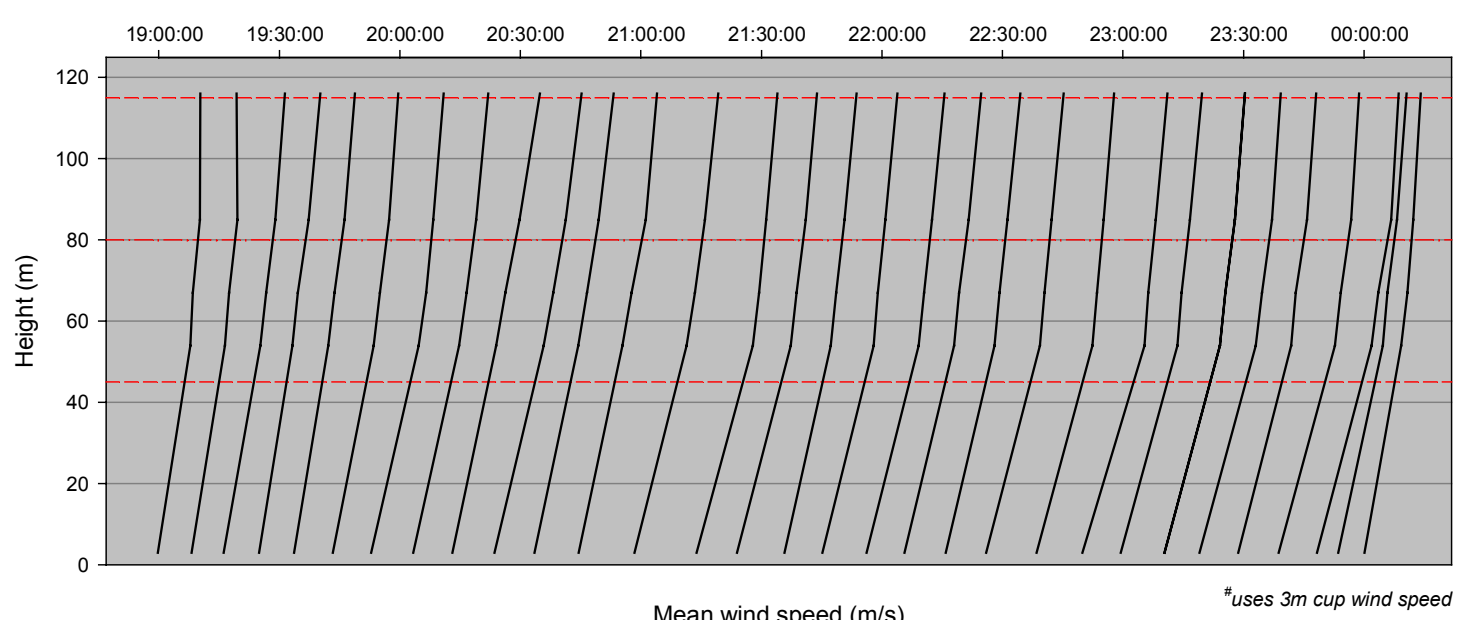

Figure 6-23. (a) Potential temperature and (b) sonic wind speed vertical profile evolutions for 17 June 2002 - PM Case 


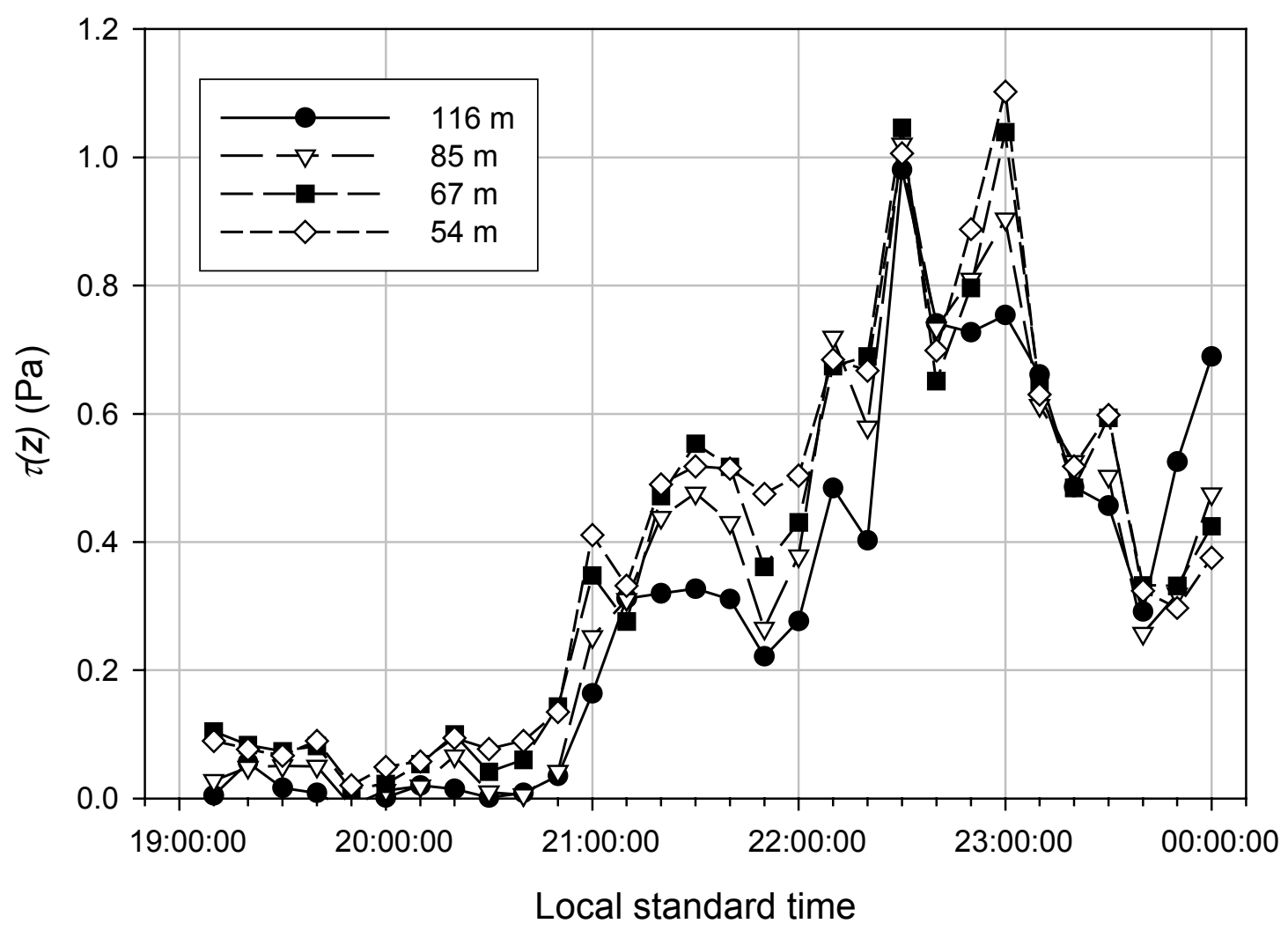

Figure 6-24. Time history of shear stress profile for 17 June 2002 - PM Case 

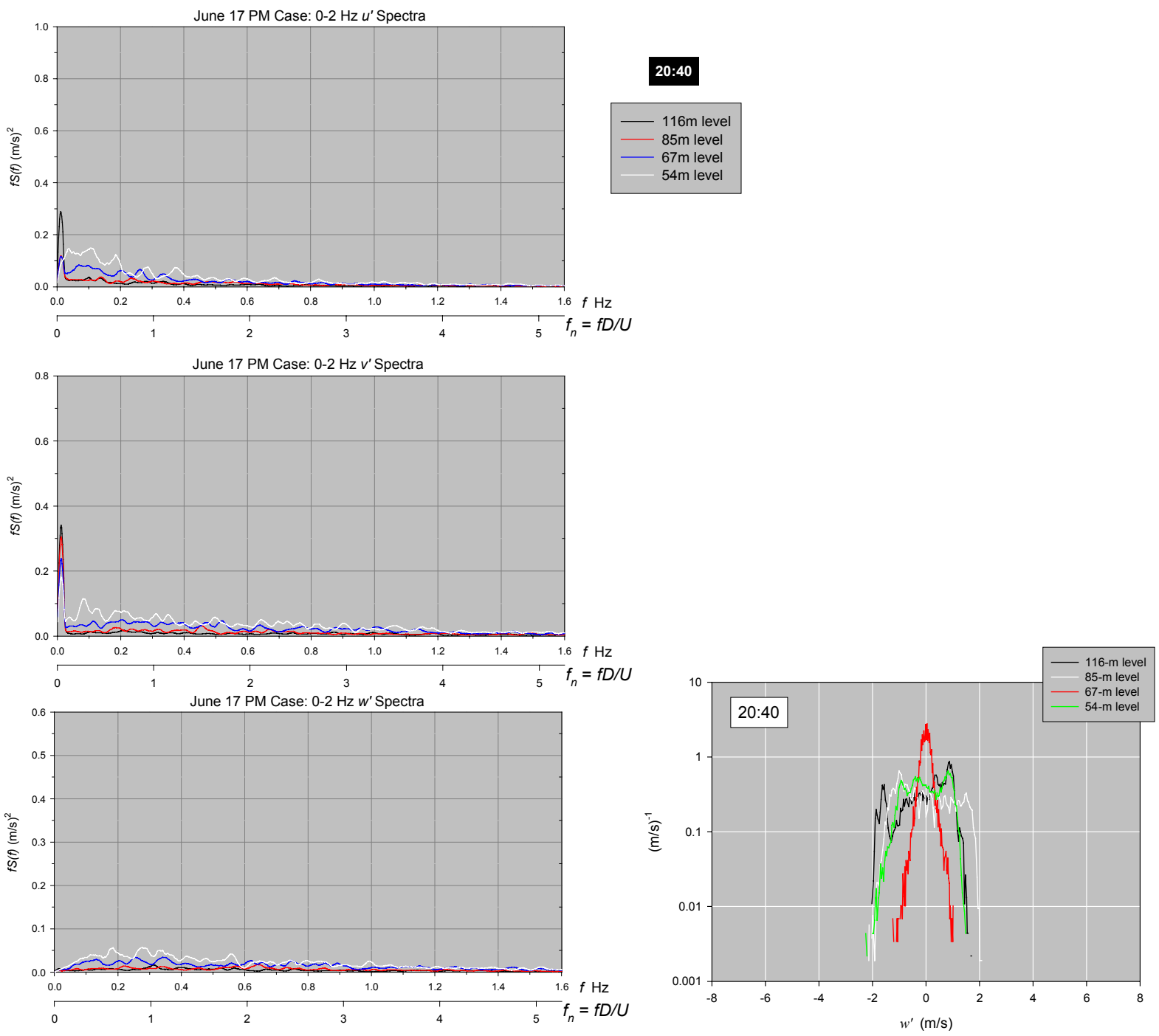

Figure 6-25a. Logarithmic spectra of $u^{\prime}, v^{\prime}$, and probability distribution of $w^{\prime}$ for 20:40 h of 17 June 2002 

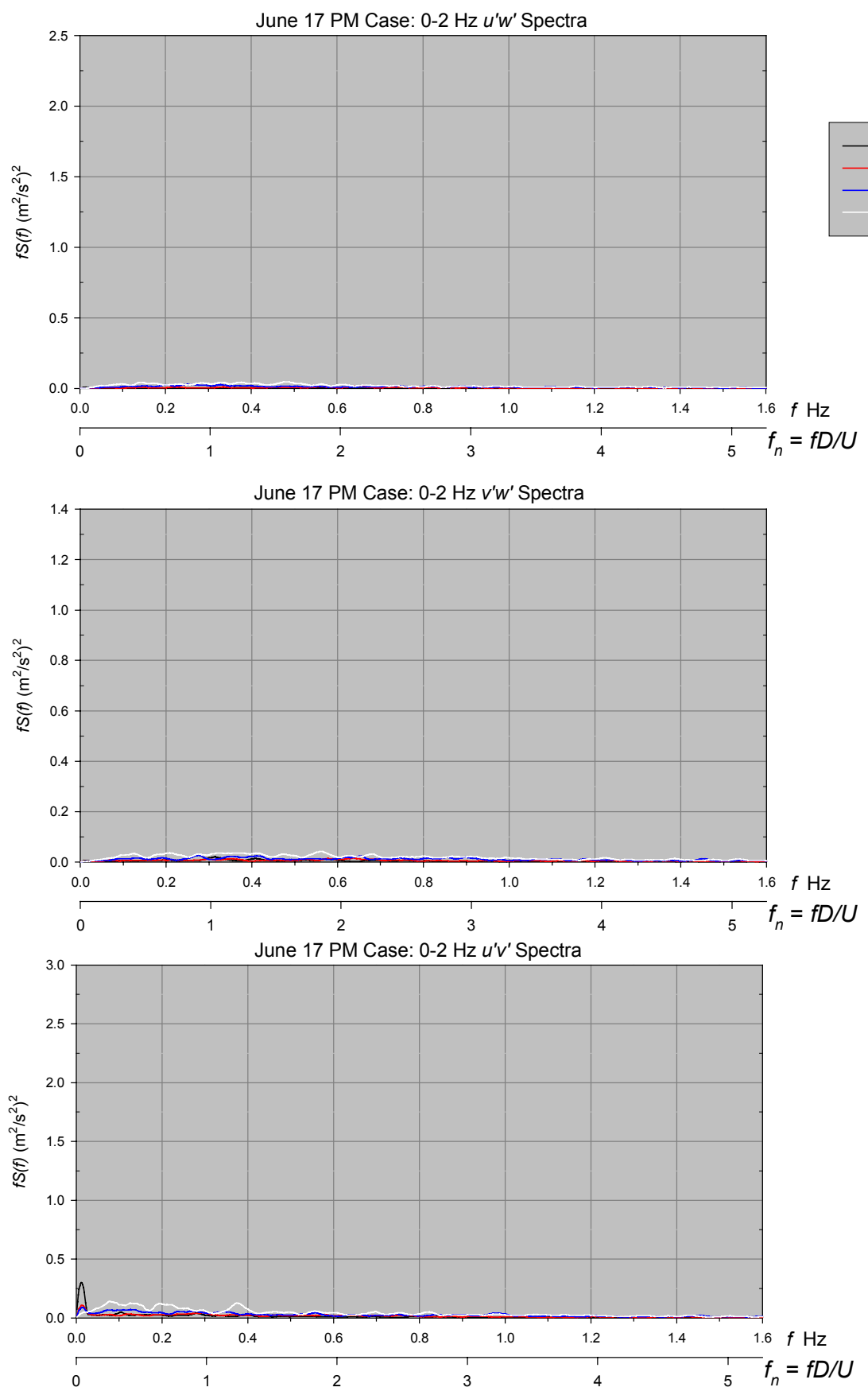

Figure 6-25b. Logarithmic spectra of $u^{\prime} w^{\prime}, u^{\prime} v^{\prime}$, and $v^{\prime} w^{\prime}$ for $20: 40 \mathrm{~h}$ of 17 June 2002 

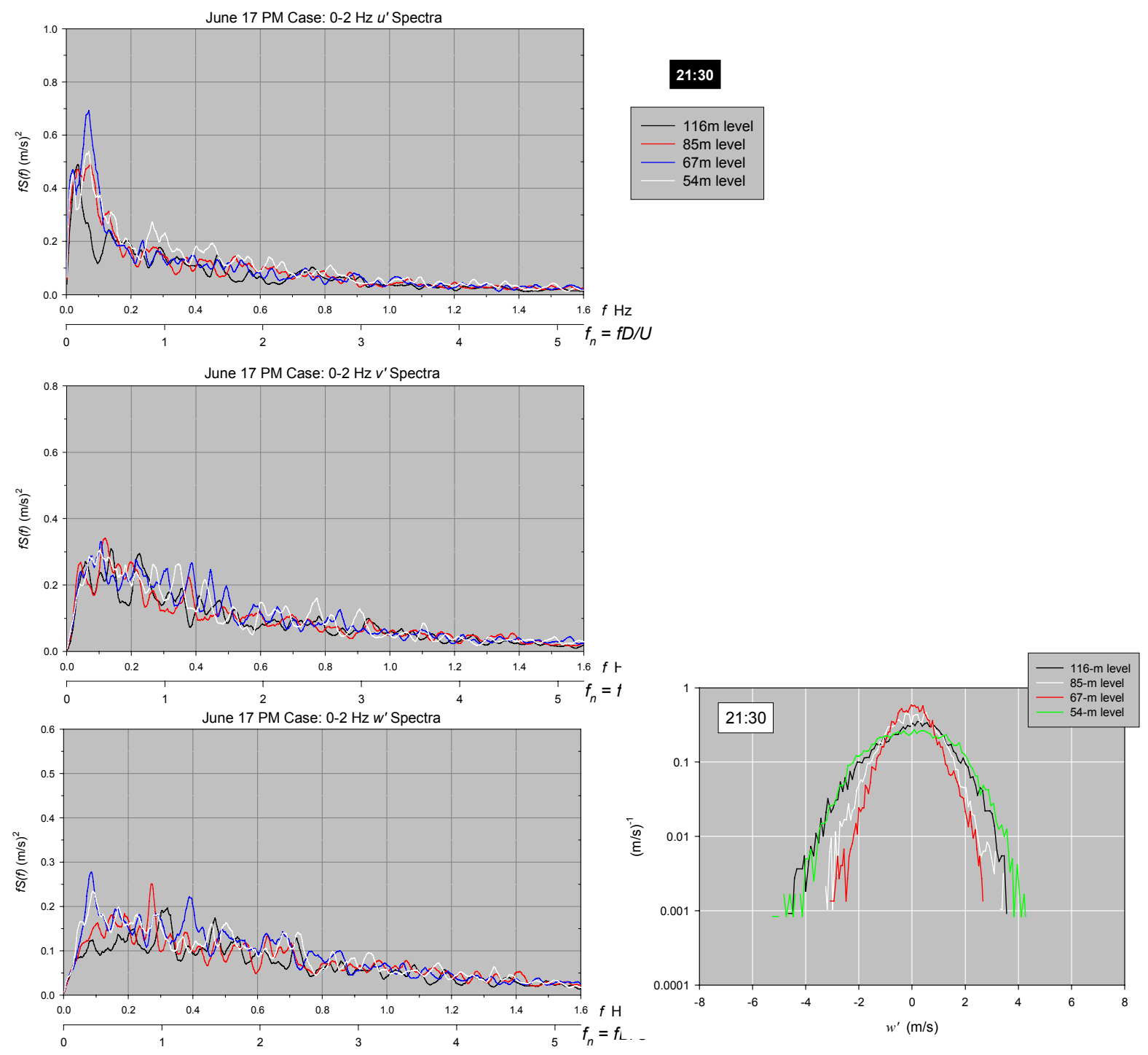

Figure 6-26a. Logarithmic spectra of $u^{\prime}, v^{\prime}$, and $w^{\prime}$ and probability distribution of $w^{\prime}$ for $21: 30 \mathrm{~h}$ of 17 June 2002 

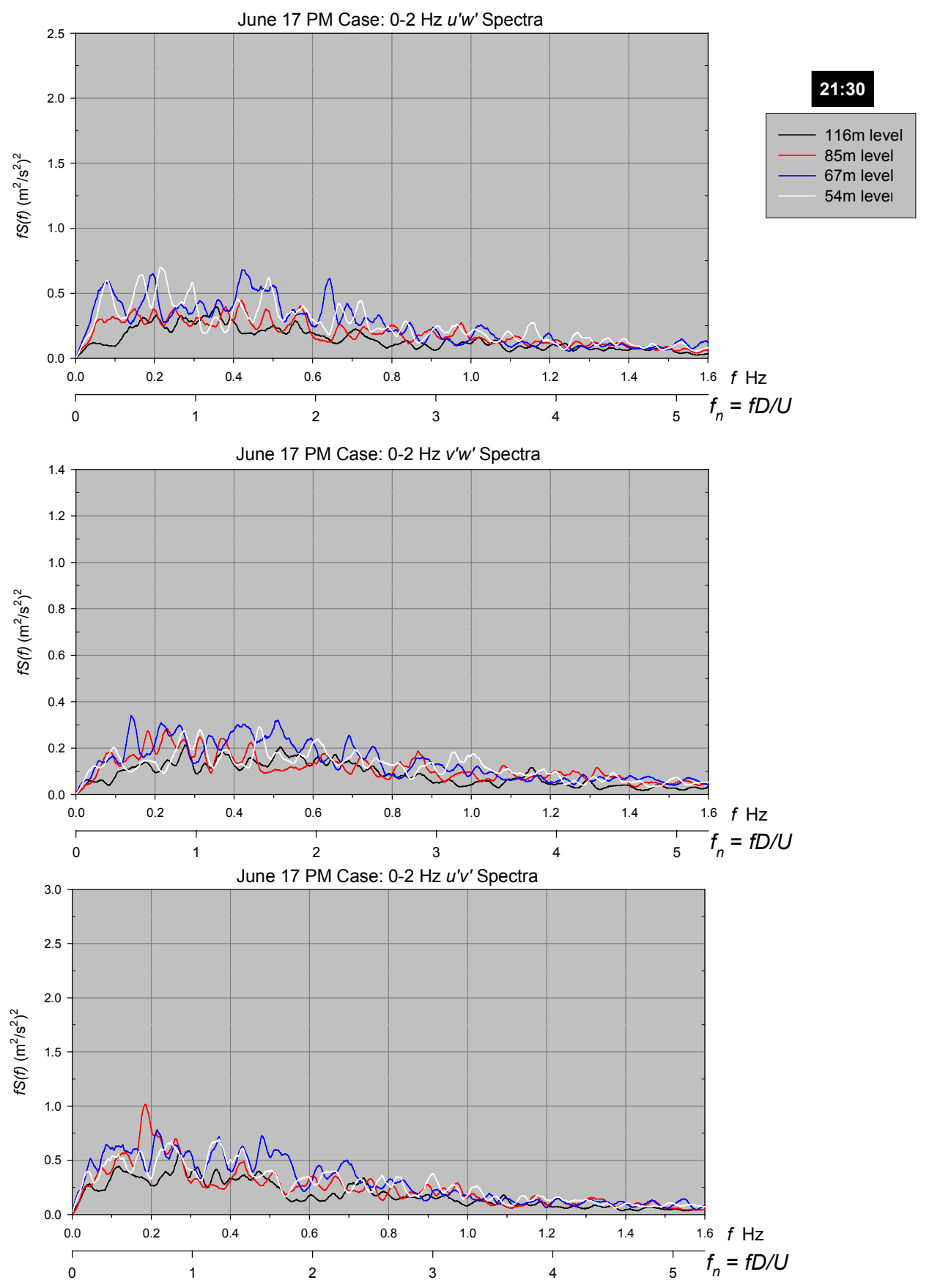

Figure 6-26b. Logarithmic spectra of $u^{\prime} w^{\prime}, u^{\prime} v^{\prime}$, and $v^{\prime} w^{\prime}$ for 21:30 h of 17 June 2002 

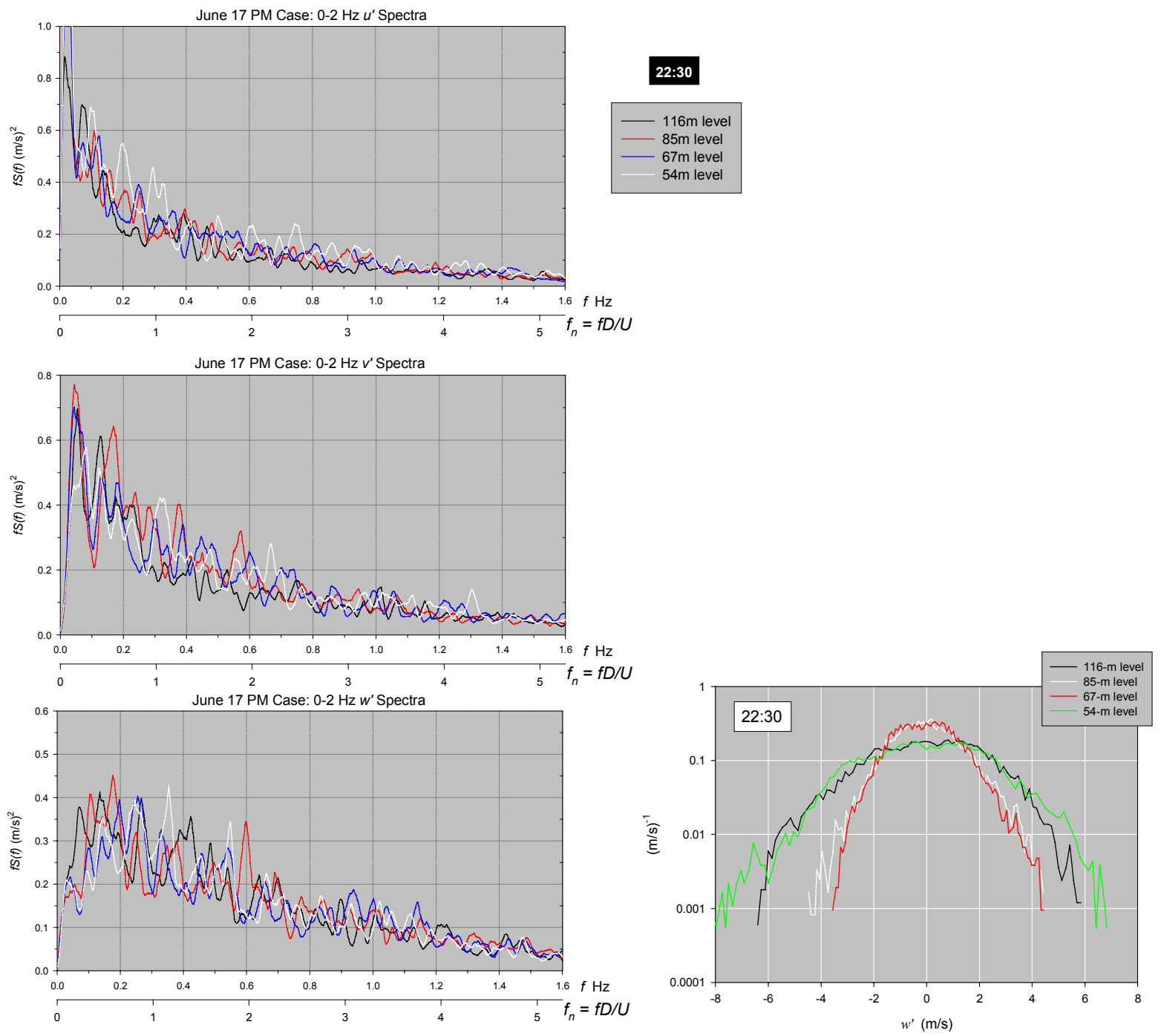

Figure 6-27a. Logarithmic spectra of $u^{\prime}, v^{\prime}$, and $w^{\prime}$ and probability distribution of $w^{\prime}$ for 22:30 h of 17 June 2002 

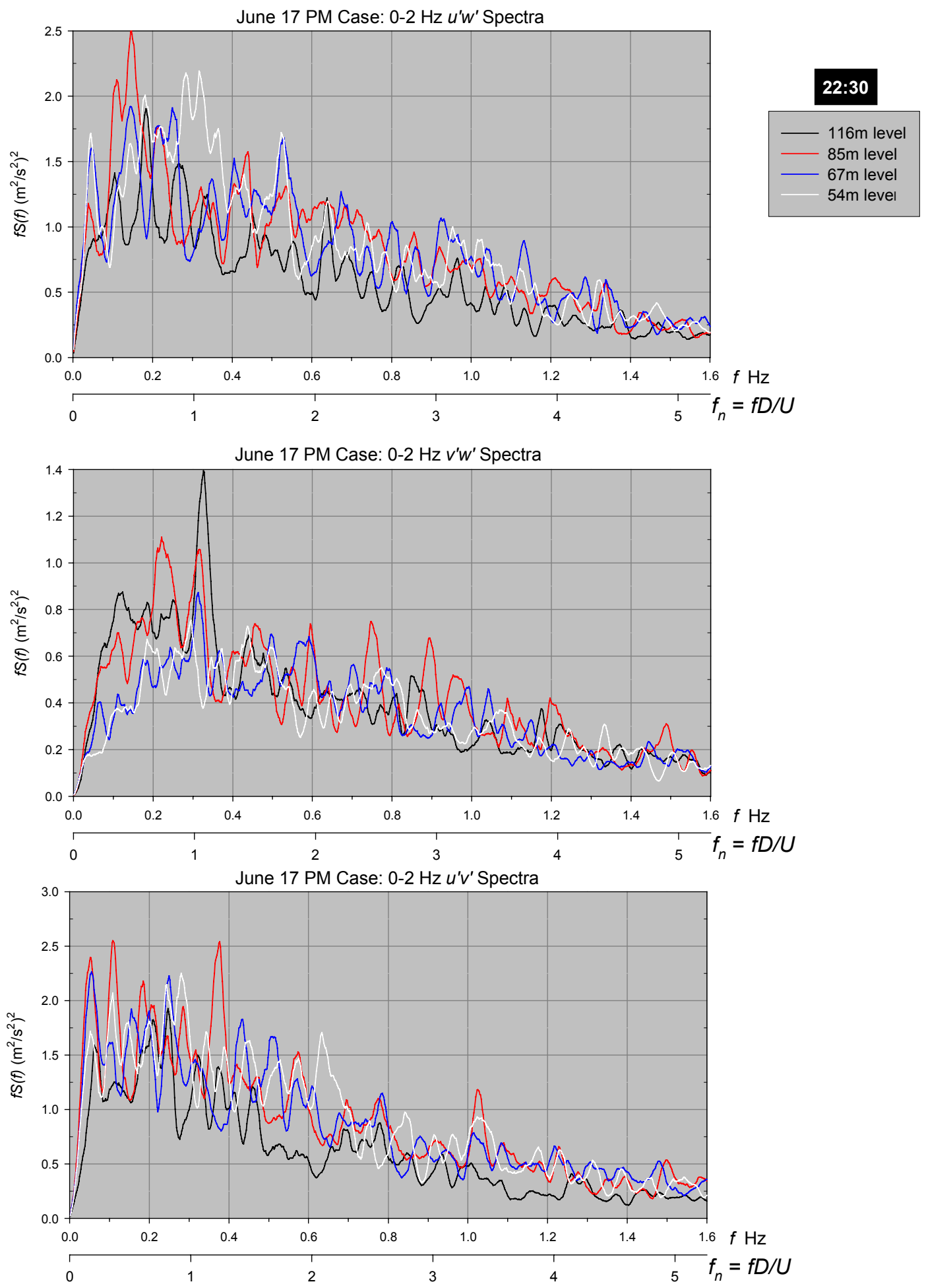

Figure 6-27b. Logarithmic spectra of $u^{\prime} w^{\prime}, u^{\prime} v^{\prime}$, and $v^{\prime} w^{\prime}$ for 22:30 h of 17 June 2002 

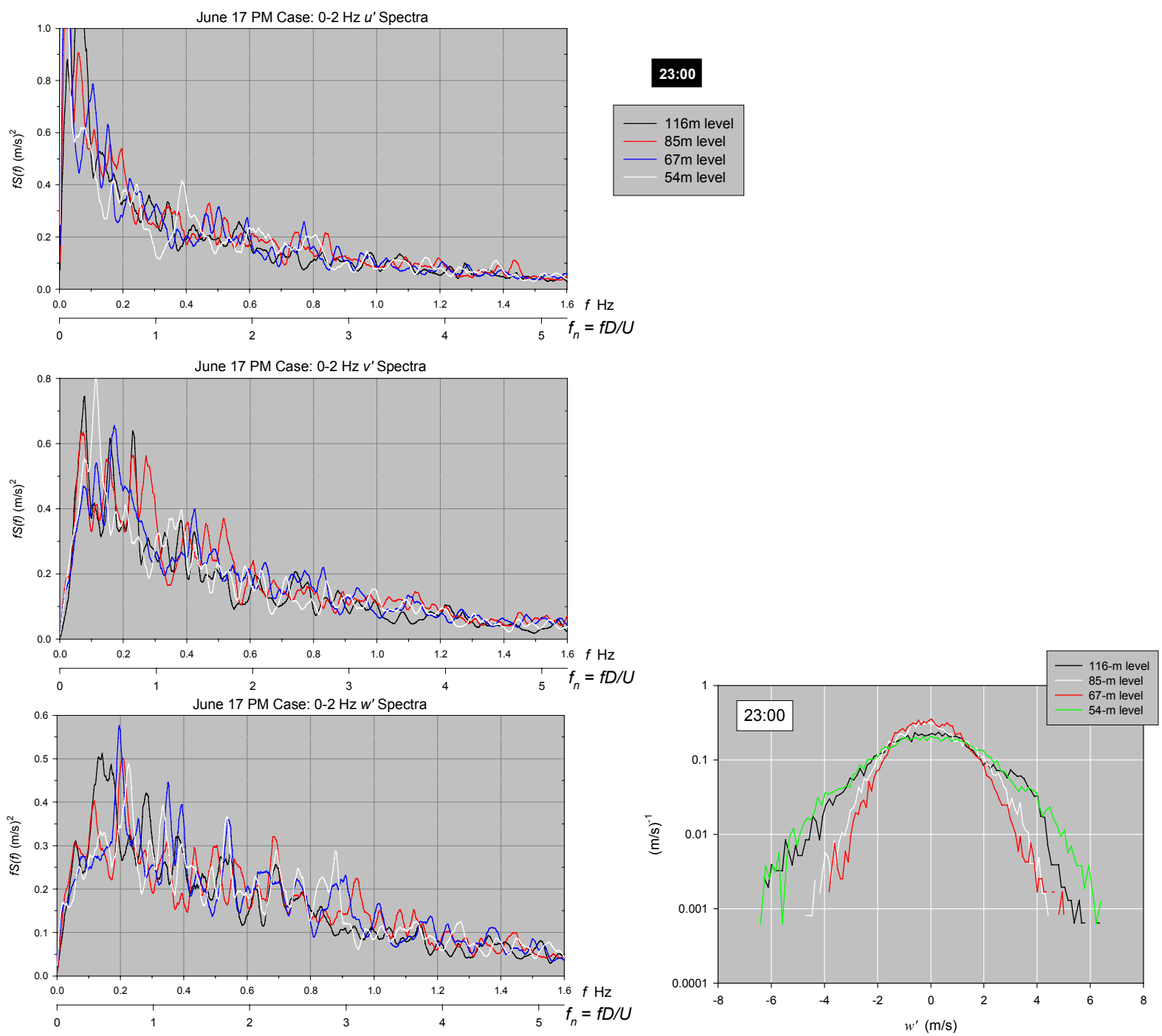

Figure 6-28a. Logarithmic spectra of $u^{\prime}, v^{\prime}$, and $w$ 'and probability distribution of $w^{\prime}$ for 23:00 h of 17 June 2002 

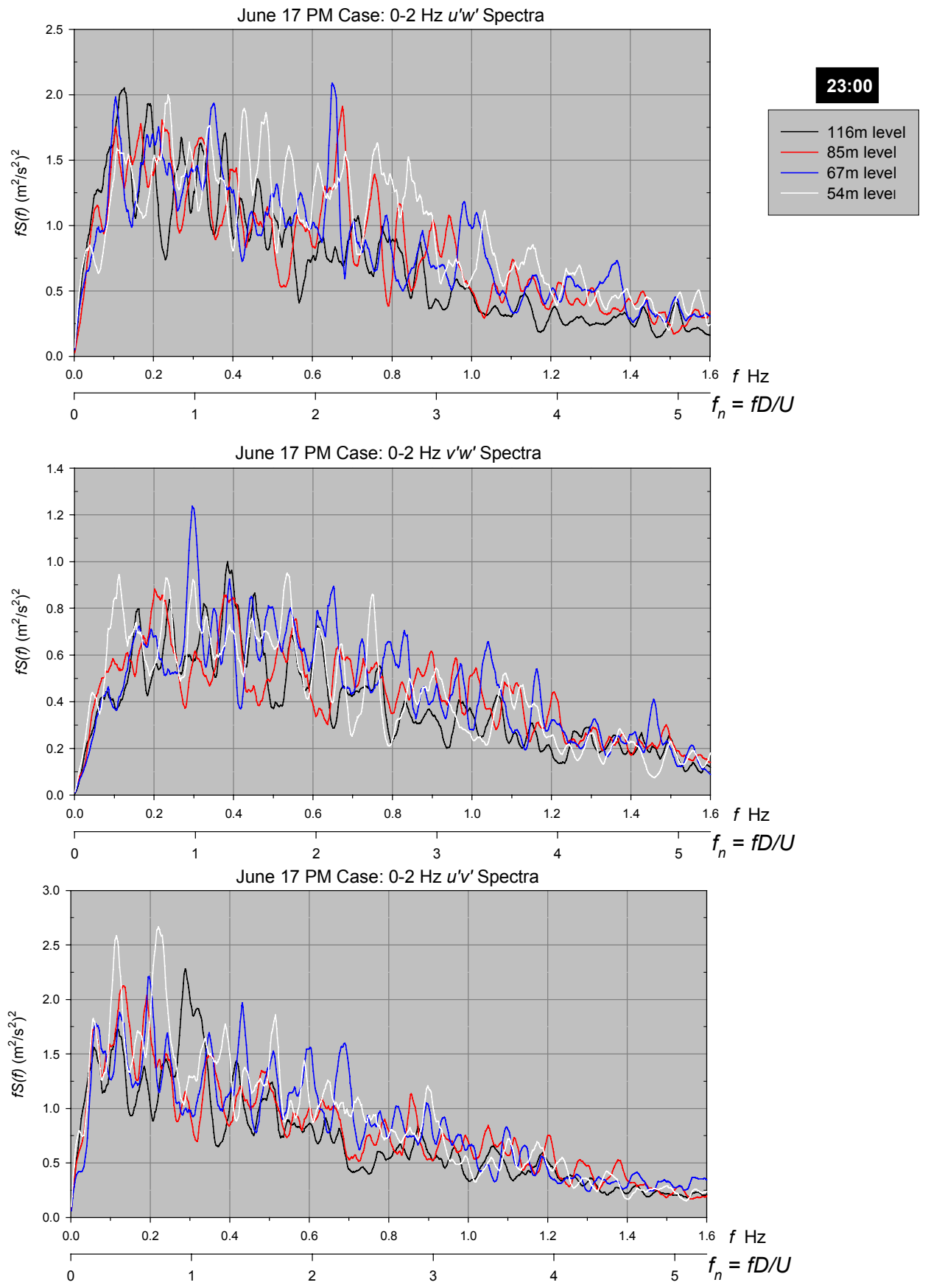

Figure 6-28b. Logarithmic spectra of $u^{\prime} w^{\prime}, u^{\prime} v^{\prime}$, and $v^{\prime} w^{\prime}$ for 23:00 h of 17 June 2002 

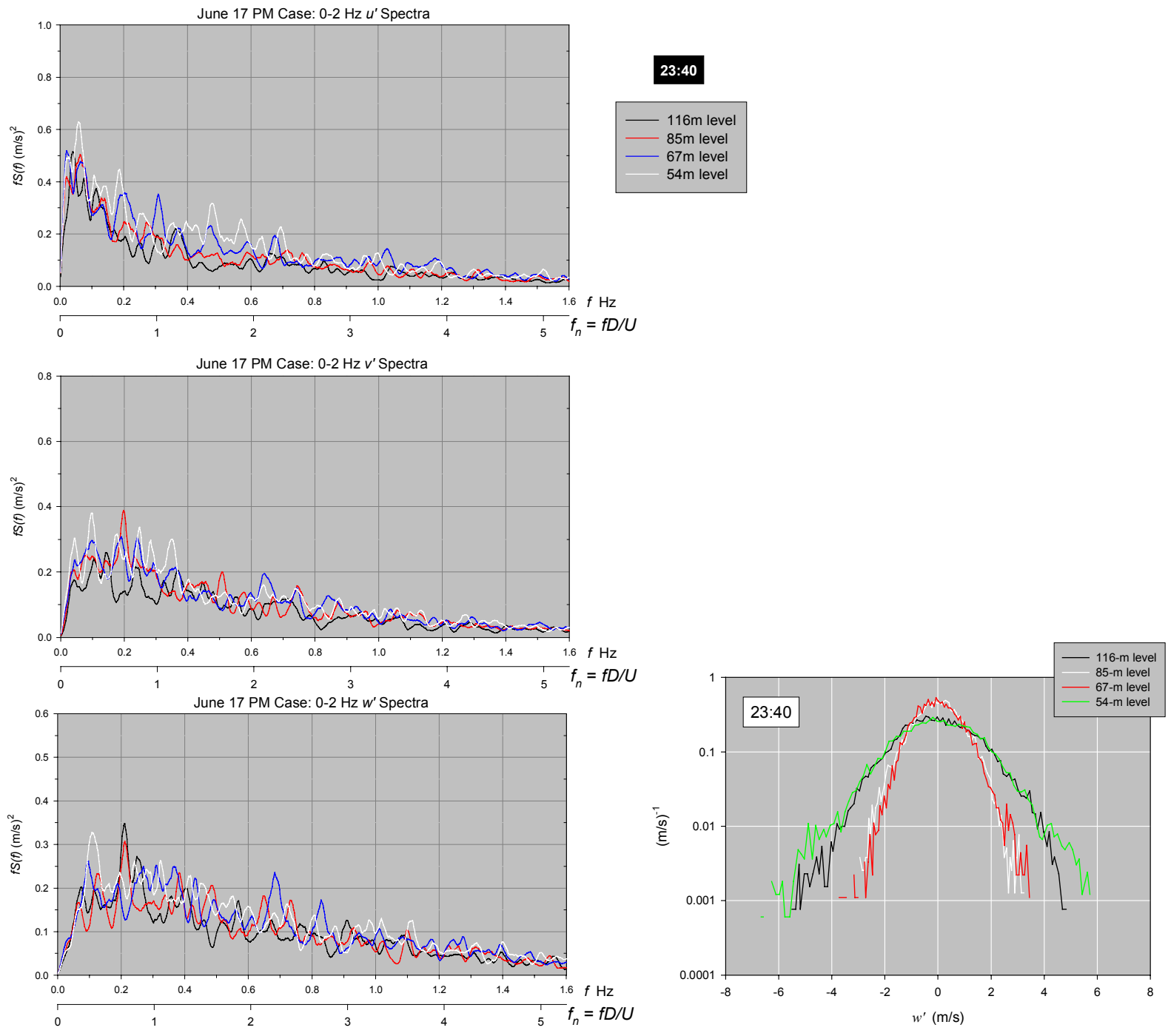

Figure 6-29a. Logarithmic spectra of $u^{\prime}, v^{\prime}$, and $w^{\prime}$ and probability distribution of $w^{\prime}$ for 23:40 h of 17 June 2002 

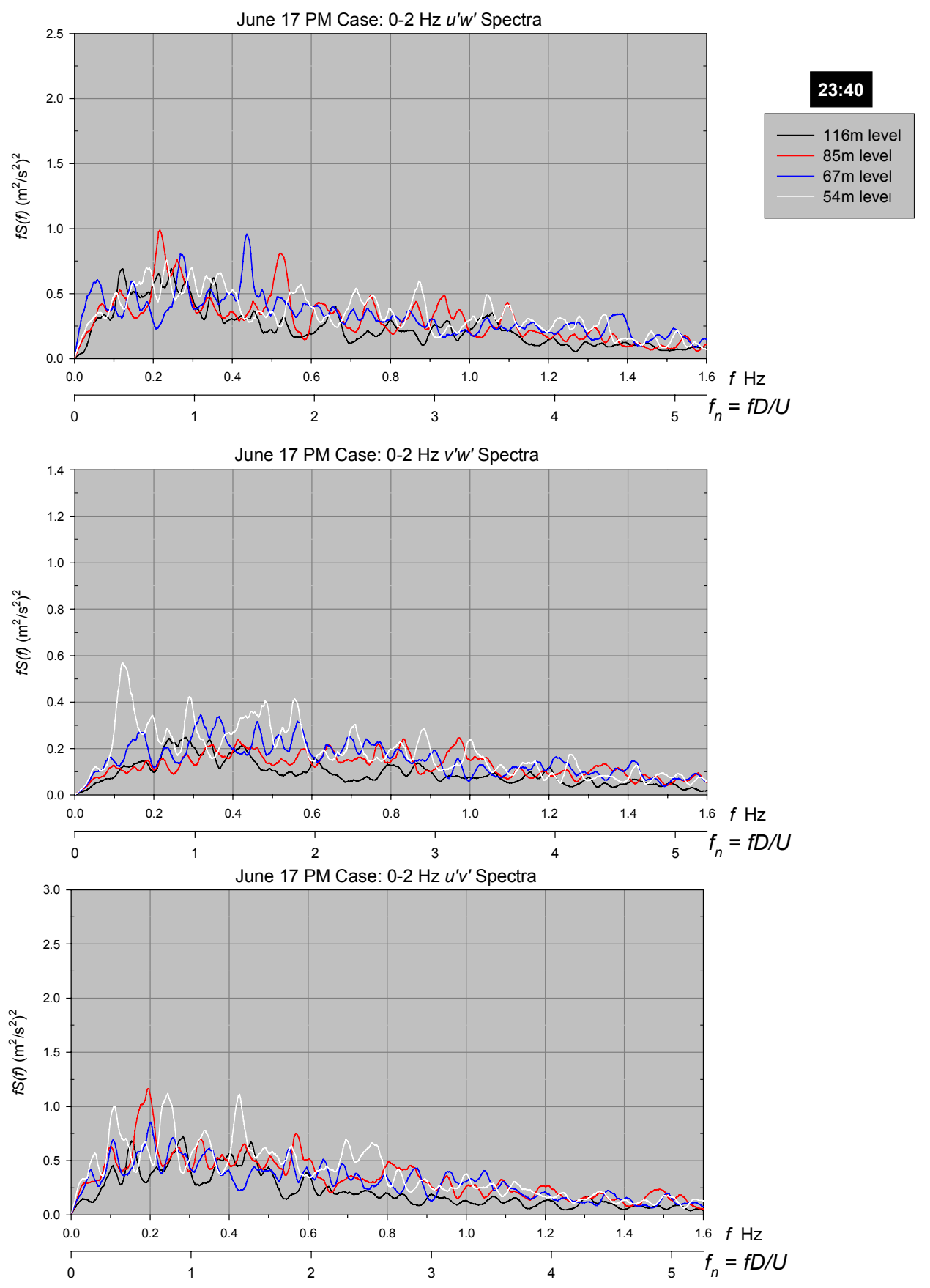

Figure 6-29b. Logarithmic spectra $u^{\prime} w^{\prime}, u^{\prime} v^{\prime}$, and $v^{\prime} w^{\prime}$ for $23: 40 \mathrm{~h}$ of 17 June 2002 

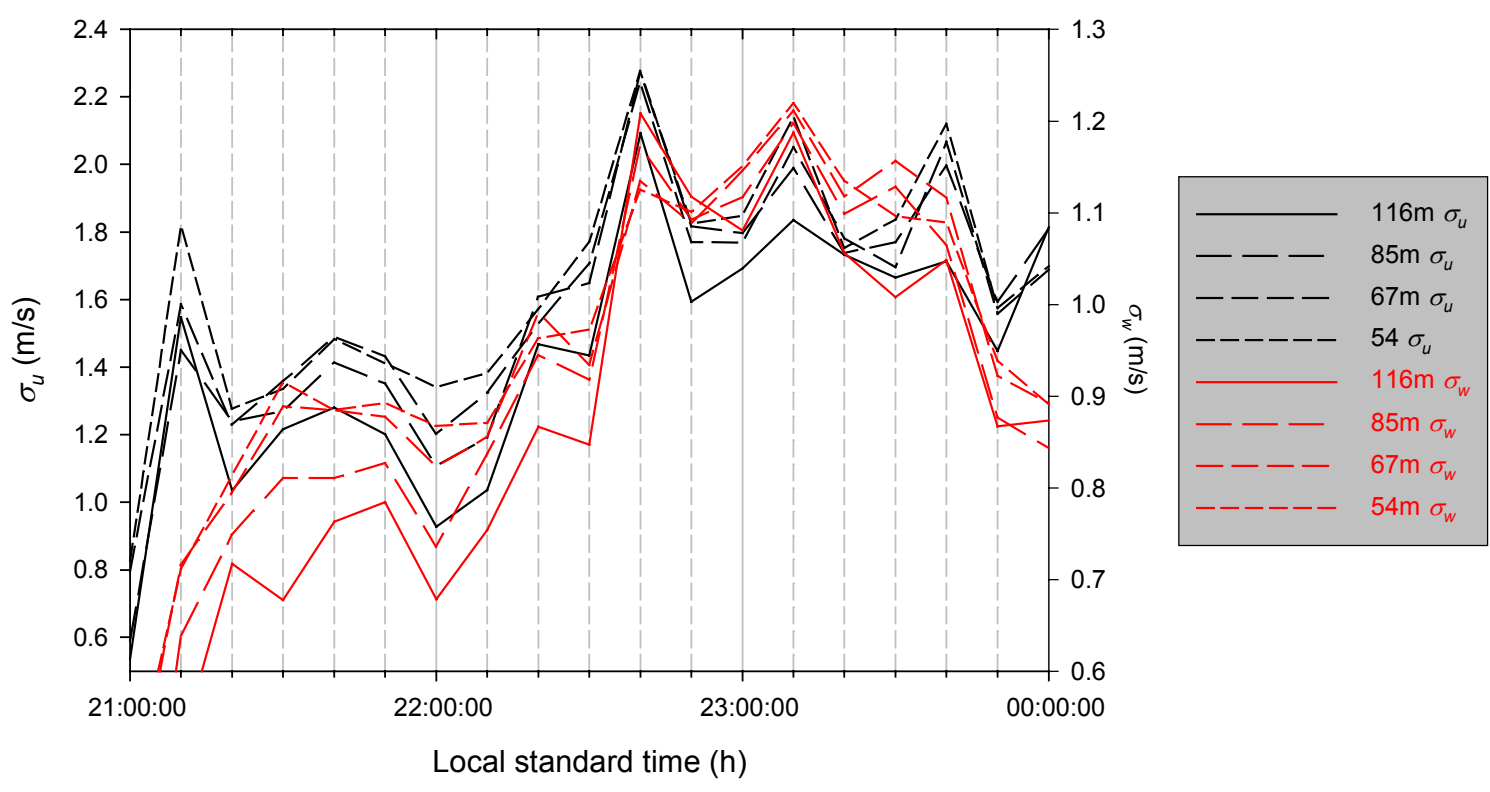

Figure 6-30. Time variation of $\sigma_{u}$ and $\sigma_{w}$ for period 21 to $24 \mathrm{~h}$ of 17 June 2002

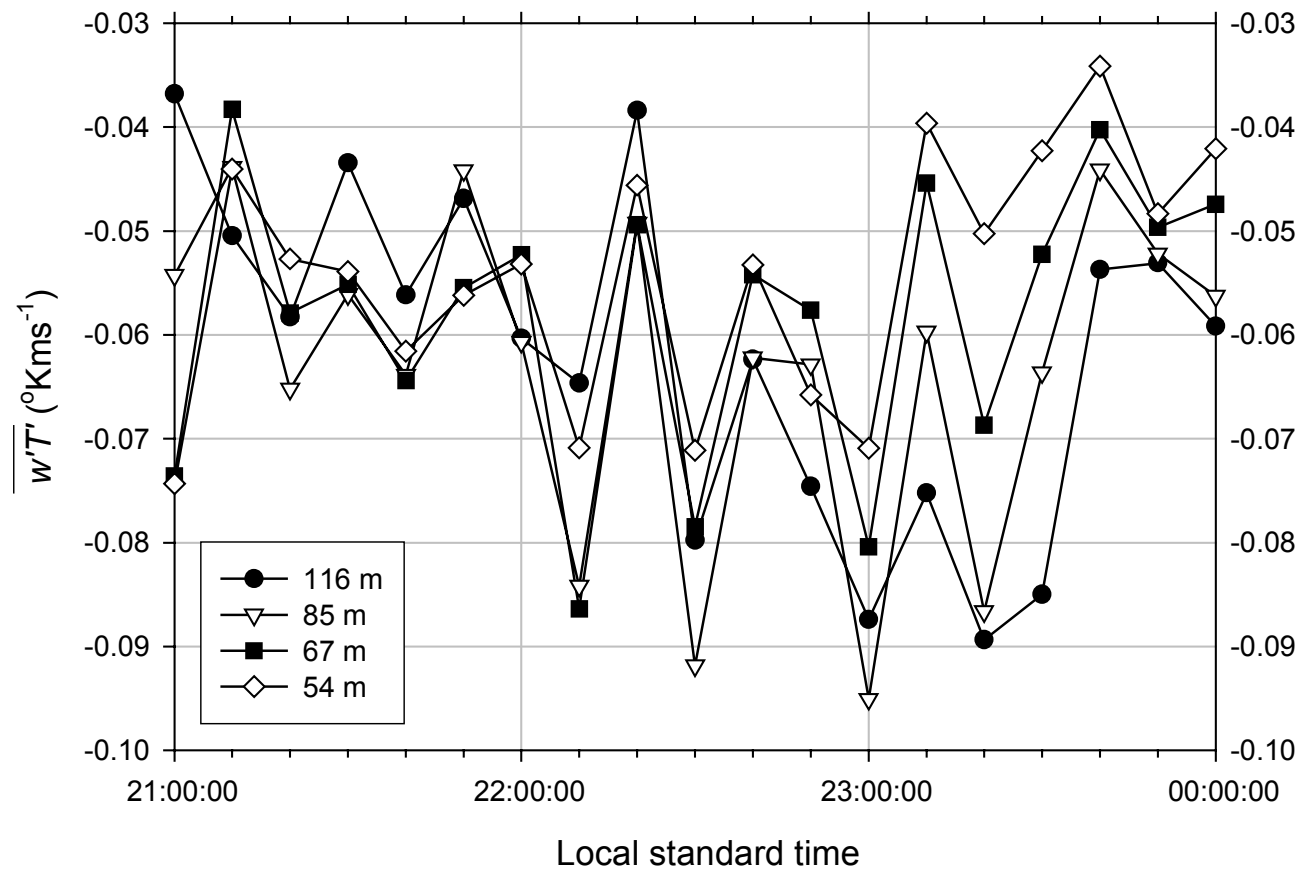

Figure 6-31. Time variation of kinematic heat fluxes $\overline{w^{\prime} T^{\prime}}$ for period 21 to $24 \mathbf{h}$ of 17 June 2002 


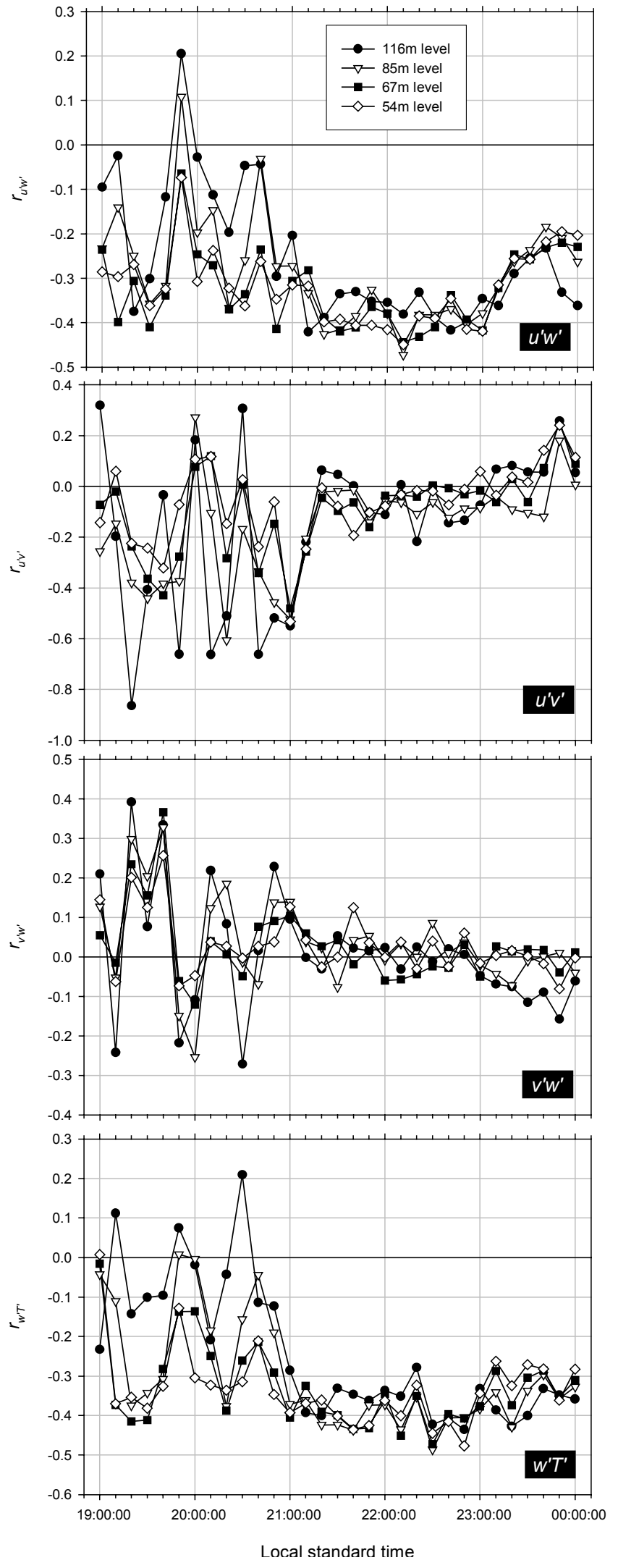

Figure 6-32. Cross-correlation coefficients for $u^{\prime} w^{\prime}, u^{\prime} v^{\prime}, v^{\prime} w^{\prime}$, and $w^{\prime} T^{\prime}$ for 17 June 2002 - PM Case 


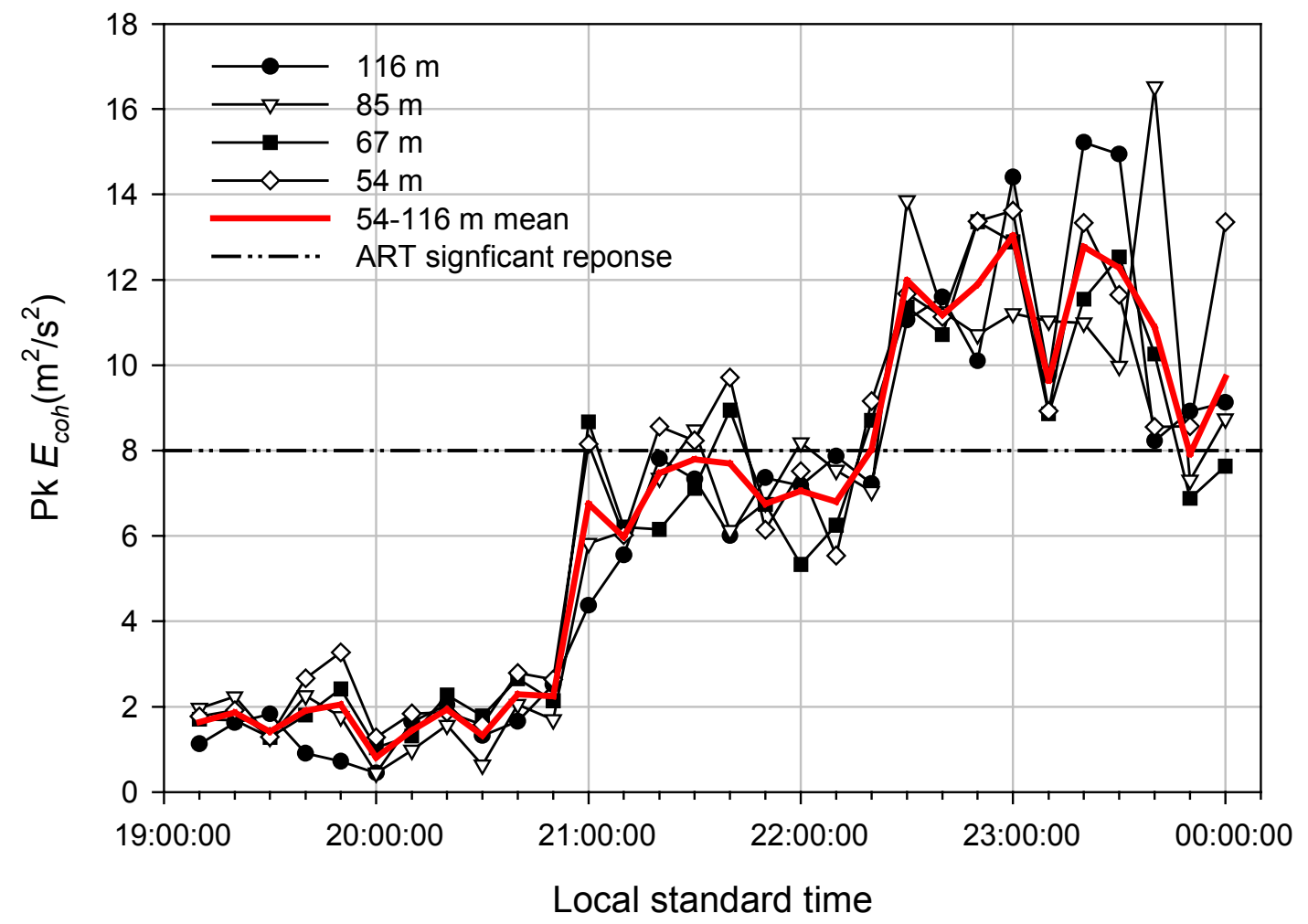

Figure 6-33. Time histories of peak coherent turbulent kinetic energy $E_{c o h}$ by height for 17 June 2002 - PM Case 


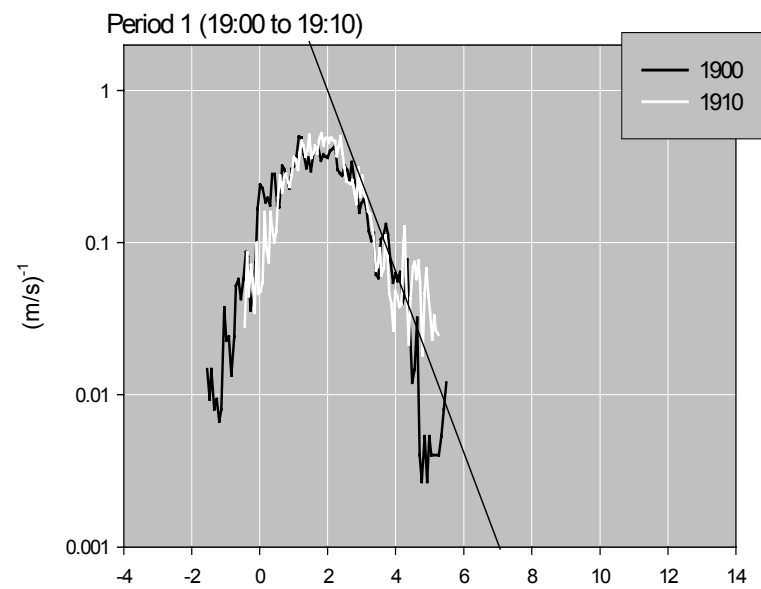

$\mathrm{m} / \mathrm{s}$
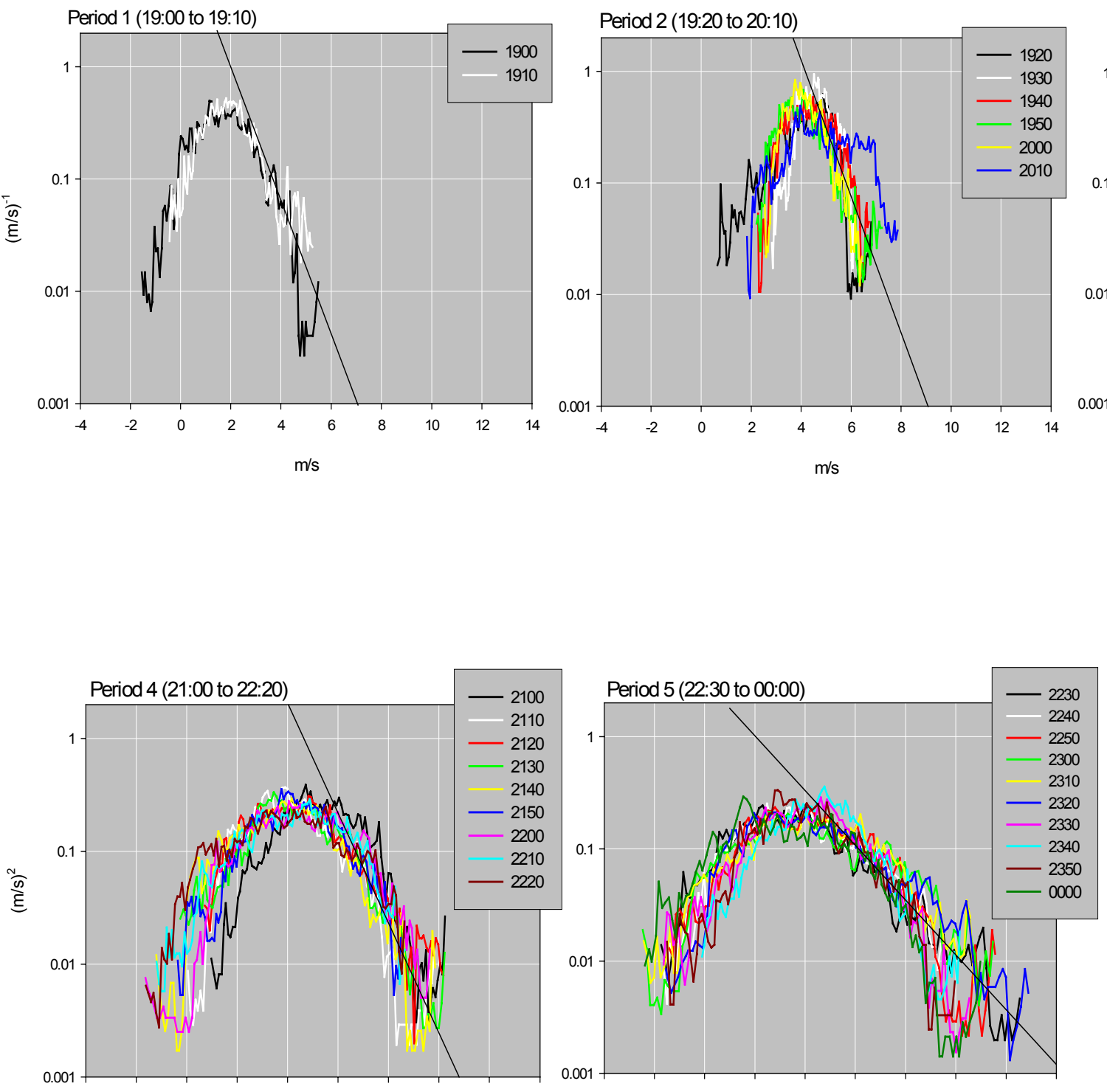

Figure 6-34. Probability density distributions of 3-s wind speed differences for 17 June 2002 - PM Case $\mathrm{m} / \mathrm{s}$

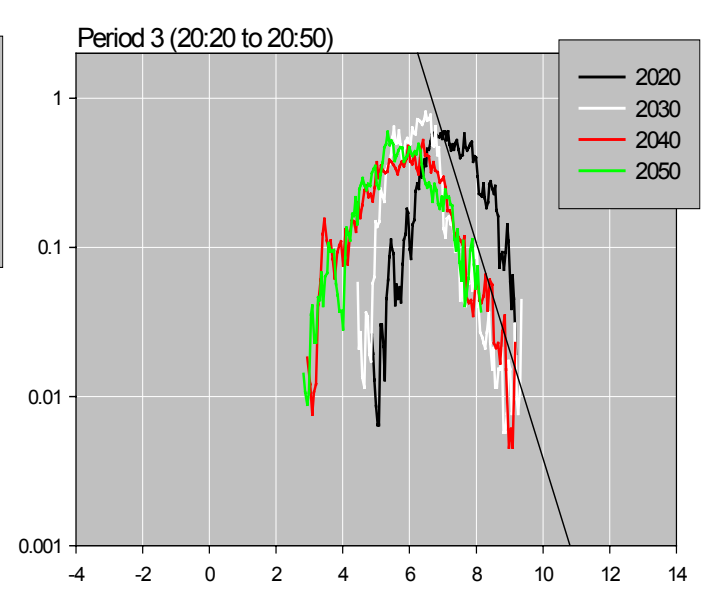

$\mathrm{m} / \mathrm{s}$

17 June 2002 PM Case

3-Sec Wind Speed Difference

Probability Density Distributions 116-54 m Height Range (Full Rotor)

(Scaled for 70.5 m rotor diameter) 

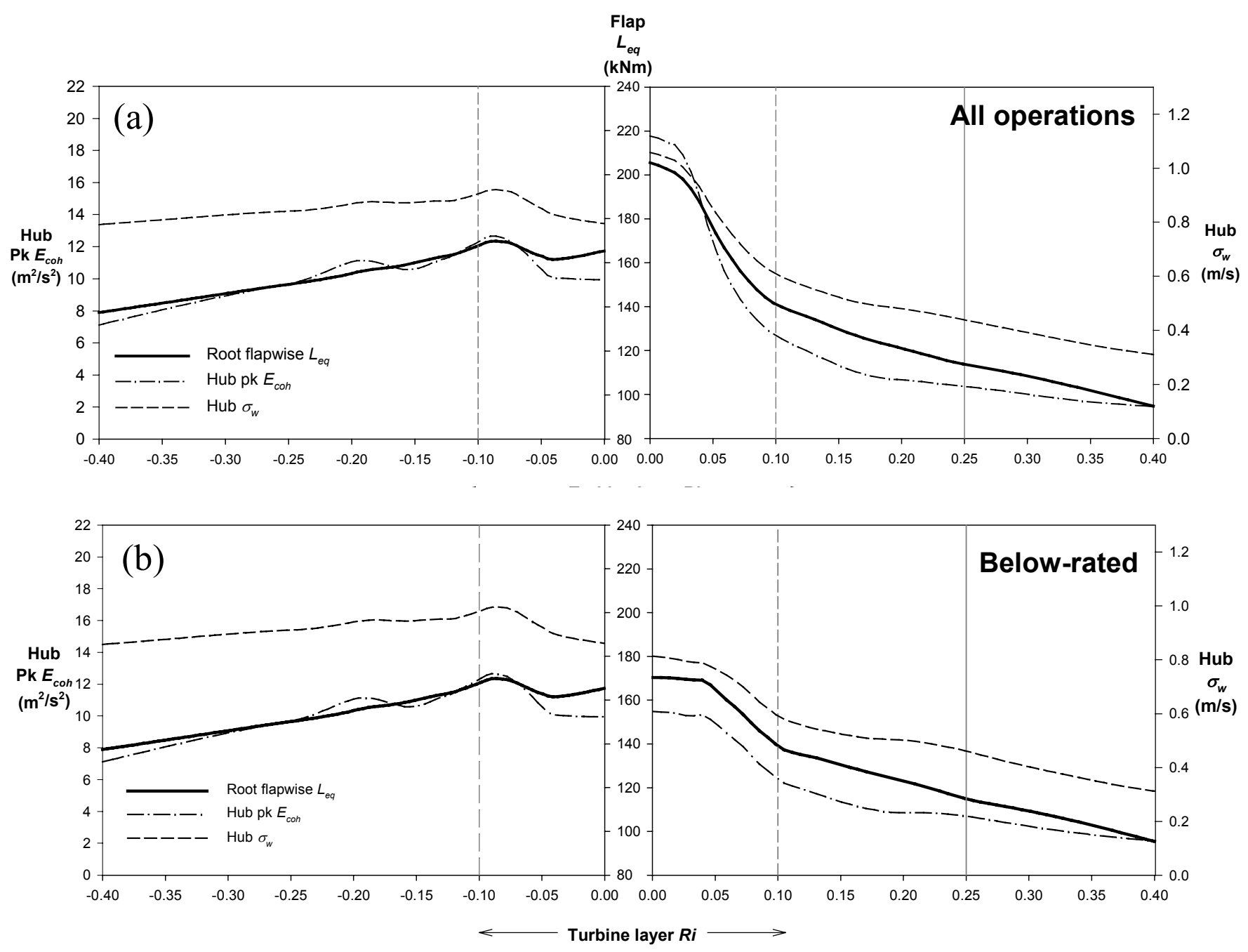

Figure 3-3. Variation of ART turbine root flapwise equivalent loads $L_{e q}$ and hub-height vertical wind speed standard deviation $\sigma_{w}$ and peak coherent turbulent kinetic energy $E_{c o h}$ as a function of the turbine-layer gradient Richardson number Ri for NWTC/LIST experiment; (a) all operations, (b) below-rated operations only 


\section{Cumulative Hours of 52m Mean Wind Speed when 52-113m Layer Shear Coefficient $>0.2$}
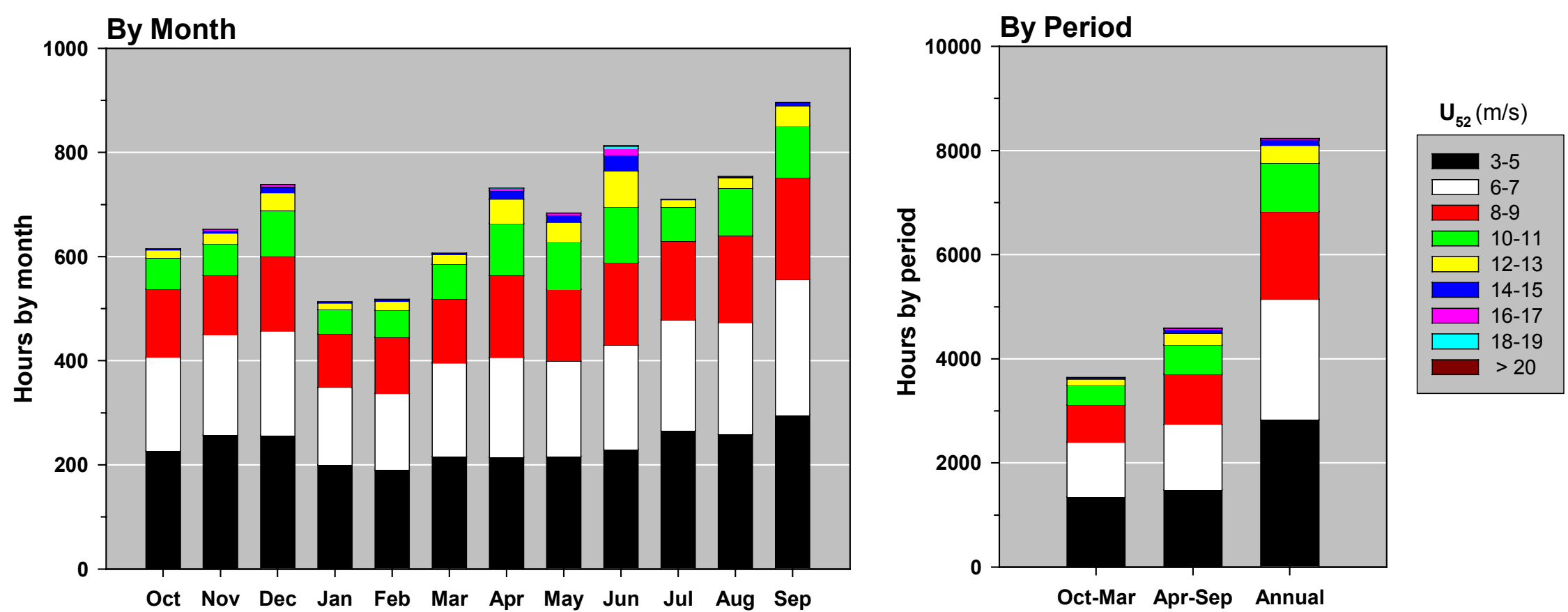

Figure 5-19. Cumulative hours of mean wind speed at 52-m when the 52- to 113-m layer shear exponent is $>0.2$ 

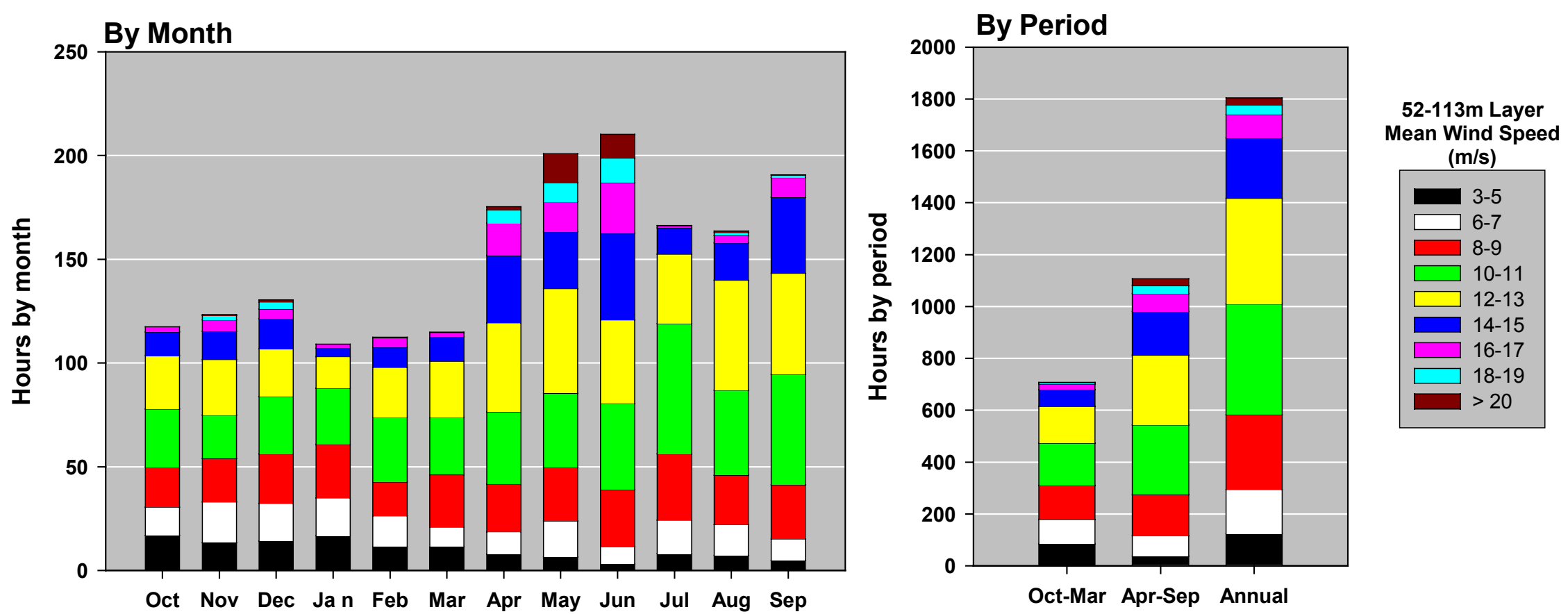

Figure 5-23. Cumulative hours of the mean wind speed at the 52- to 113-m layer when $R i$ is within critical range $(0<R i<0.25)$ 

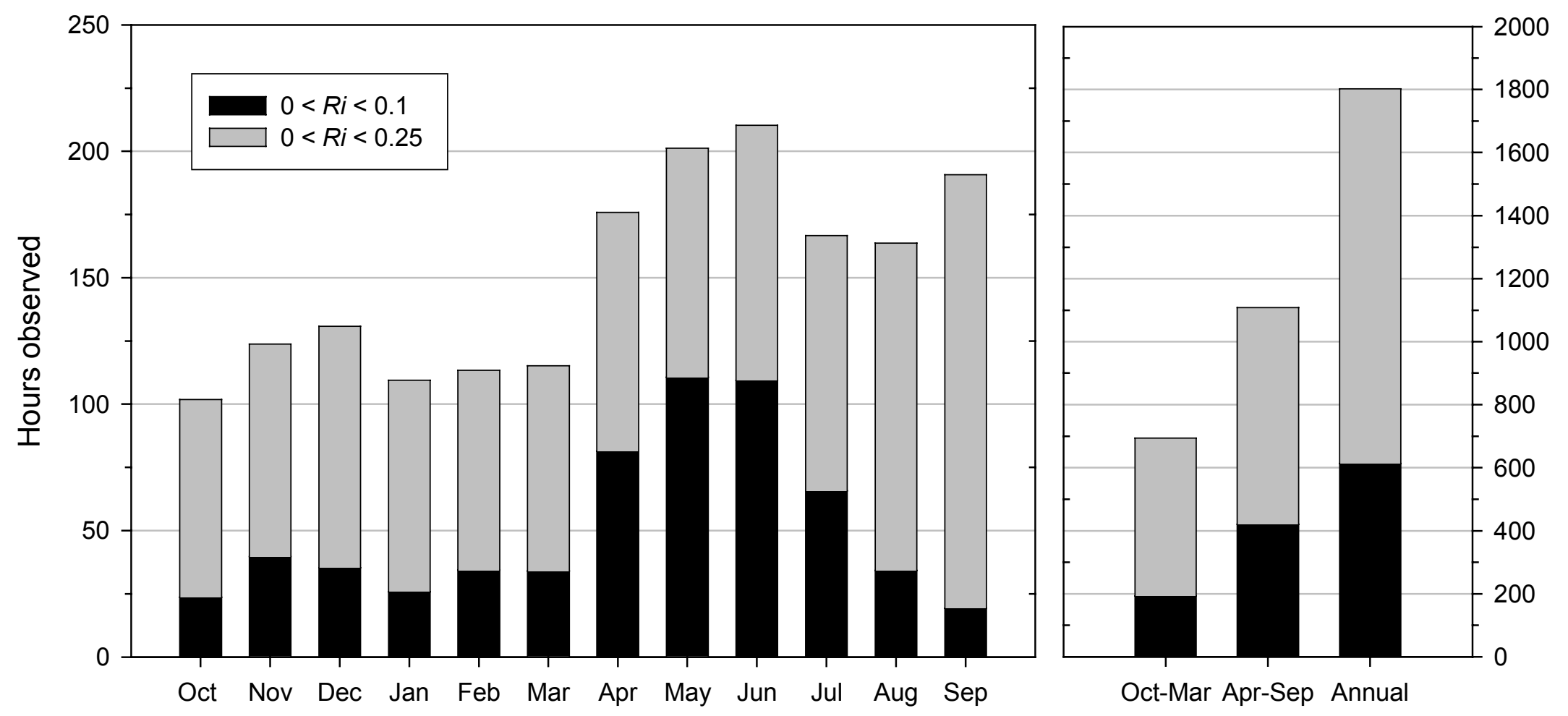

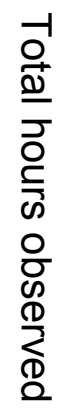

Figure 5-24. Observed monthly critical stability conditions distributions in the 52- to 113-m layer when mean wind speed at $52-\mathrm{m}$ is $>3 \mathrm{~m} / \mathrm{s}$ 


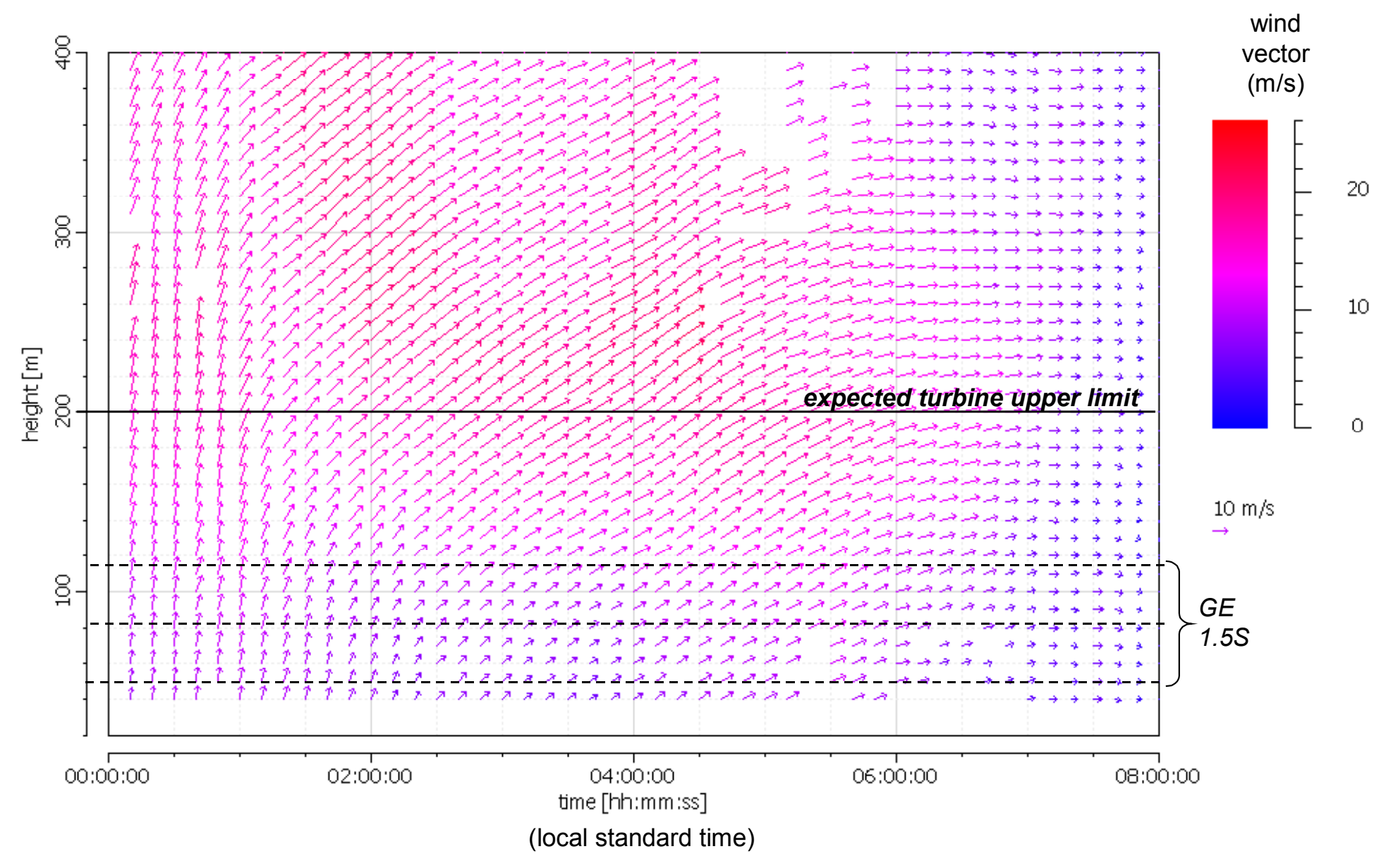

Figure 6-1. SODAR-derived 10-minute mean wind vector profiles for 00 to $08 \mathrm{~h}$ LST on 17 June 2002 


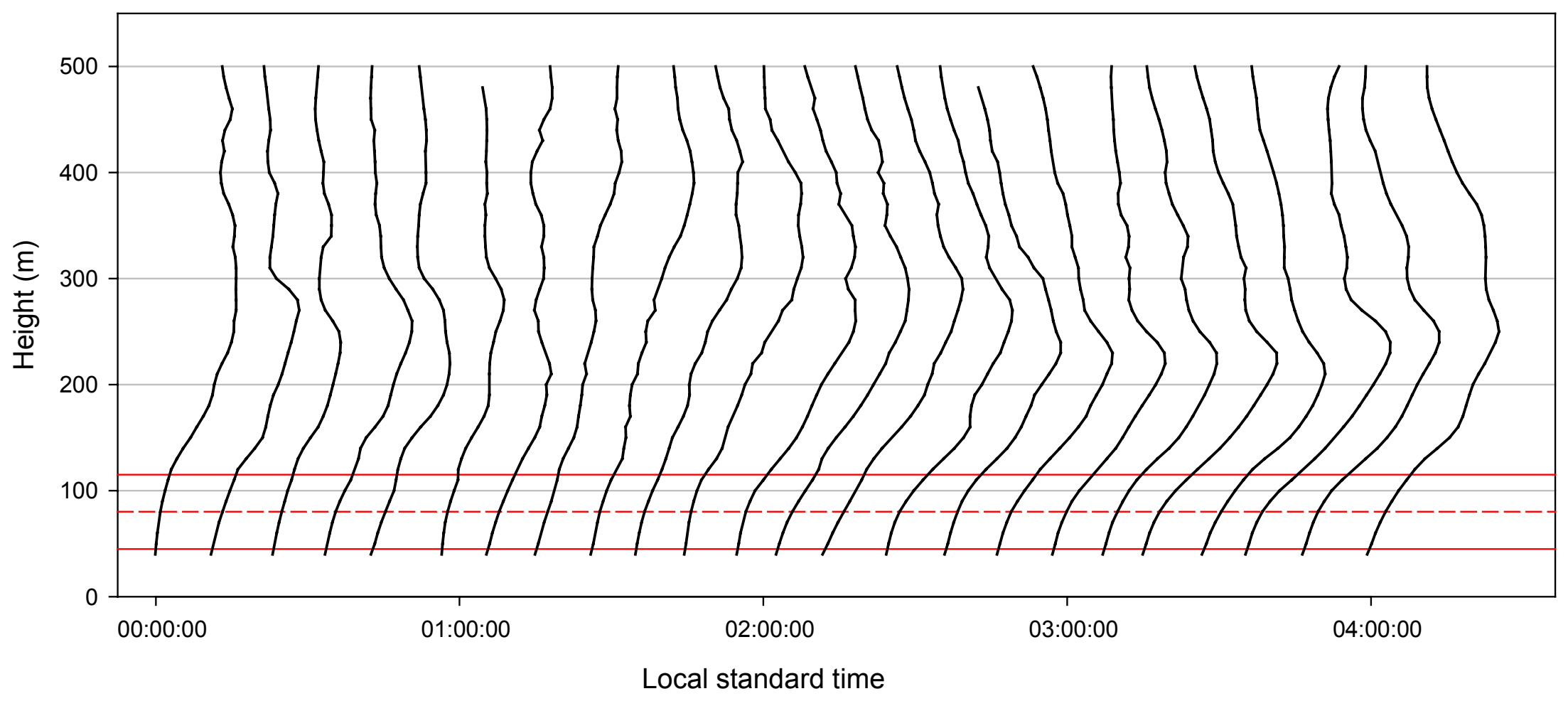

Figure 6-5. SODAR-derived wind profile evolution for 17 June 2002 - AM Case 


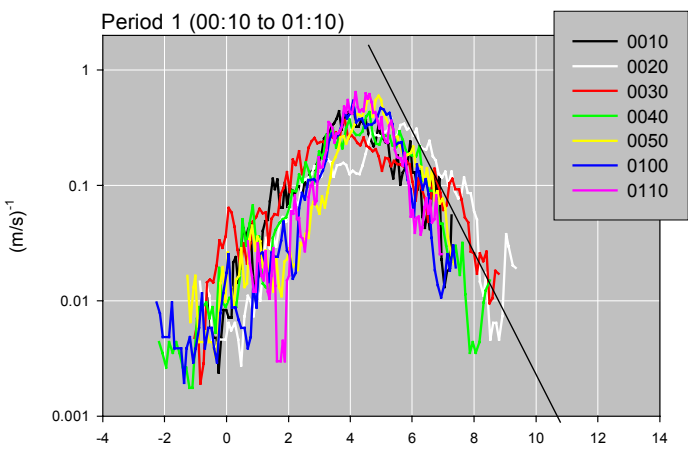

$\mathrm{m} / \mathrm{s}$

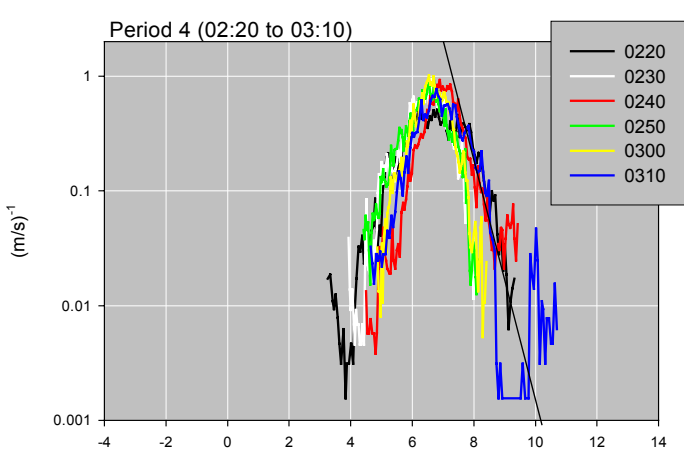

$\mathrm{m} / \mathrm{s}$

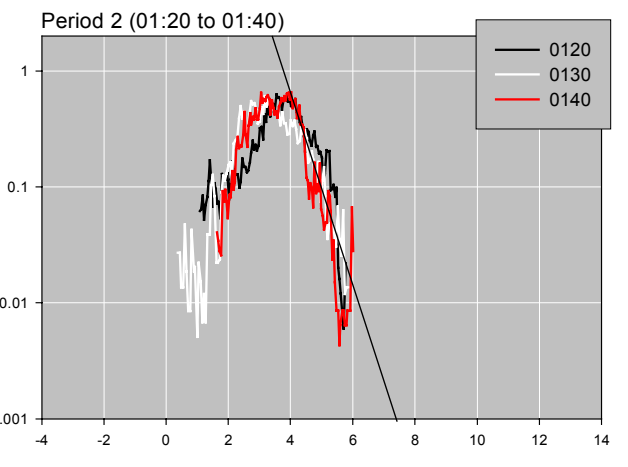

$\mathrm{m} / \mathrm{s}$

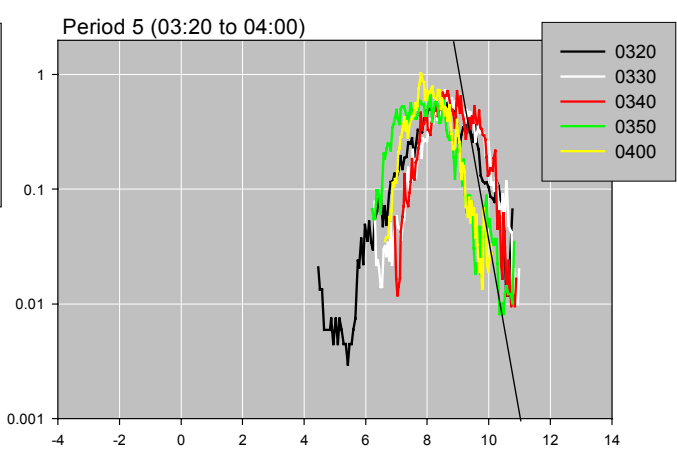

$\mathrm{m} / \mathrm{s}$

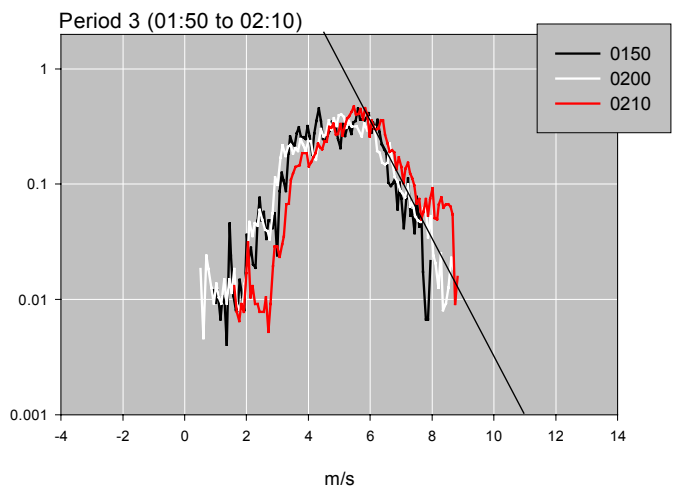

17 June 2002 AM Case

3-Sec Wind Speed Difference

Probability Density Distributions

116-54 m Height Range (Full Rotor)

(Scaled for $70.5 \mathrm{~m}$ rotor diameter)

Figure 6-12. Probability density distributions of 3-s wind speed differences for 17 June 2002 - AM Case 


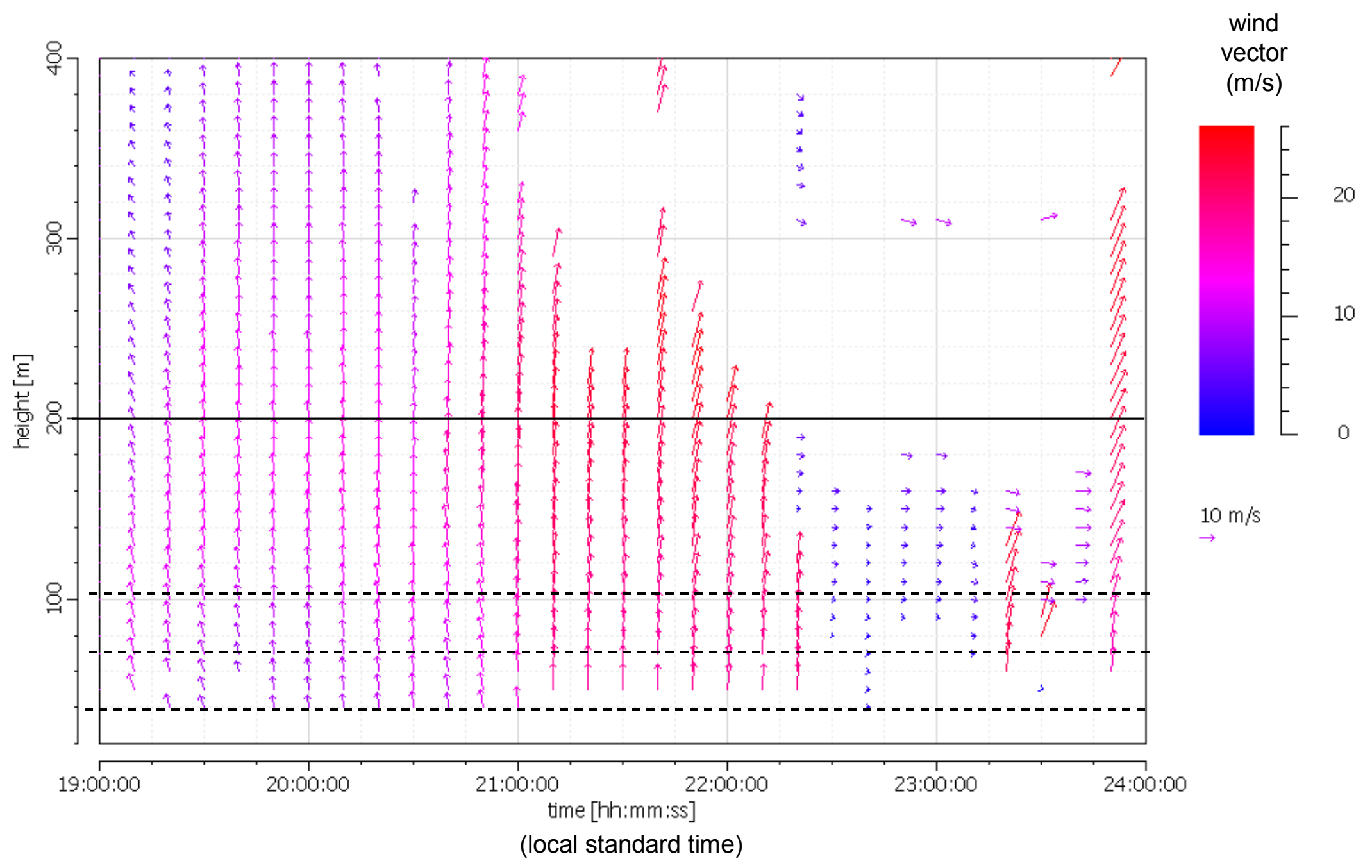

Figure 6-14. SODAR-derived 10-minute mean wind vector profiles for 19 to $24 \mathrm{~h}$ LST on 17 June 2002 


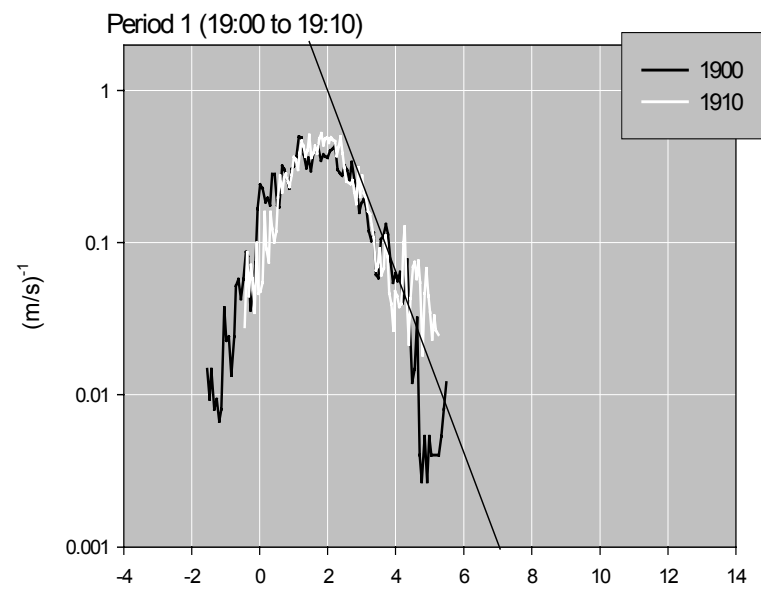

$\mathrm{m} / \mathrm{s}$
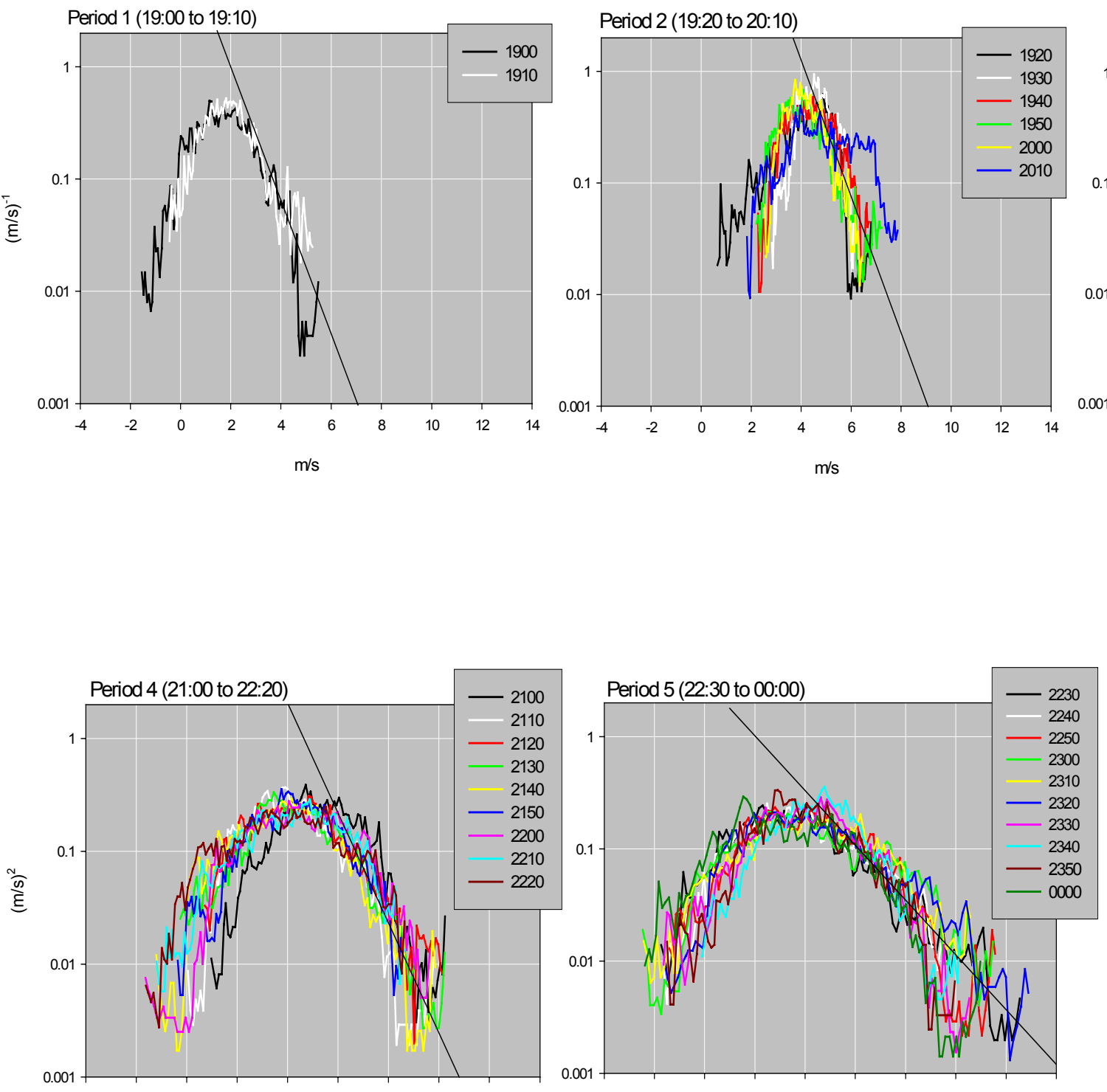

Figure 6-34. Probability density distributions of 3-s wind speed differences for 17 June 2002 - PM Case $\mathrm{m} / \mathrm{s}$

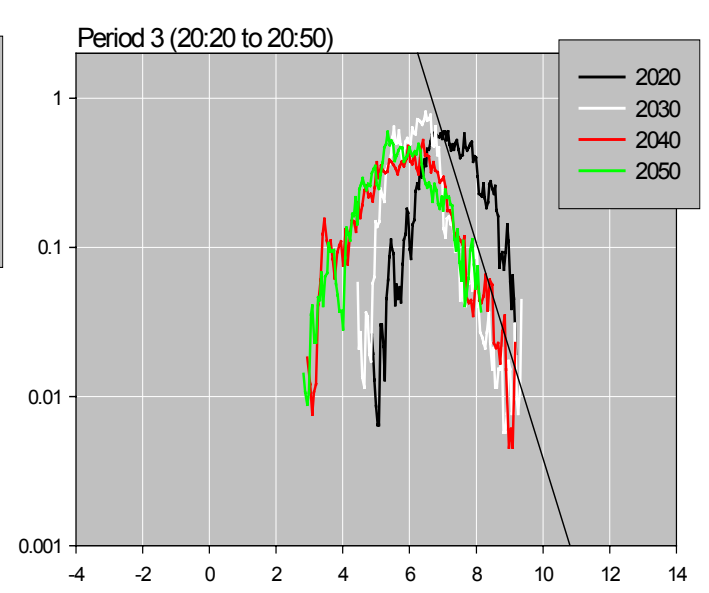

$\mathrm{m} / \mathrm{s}$

17 June 2002 PM Case

3-Sec Wind Speed Difference

Probability Density Distributions 116-54 m Height Range (Full Rotor)

(Scaled for 70.5 m rotor diameter) 


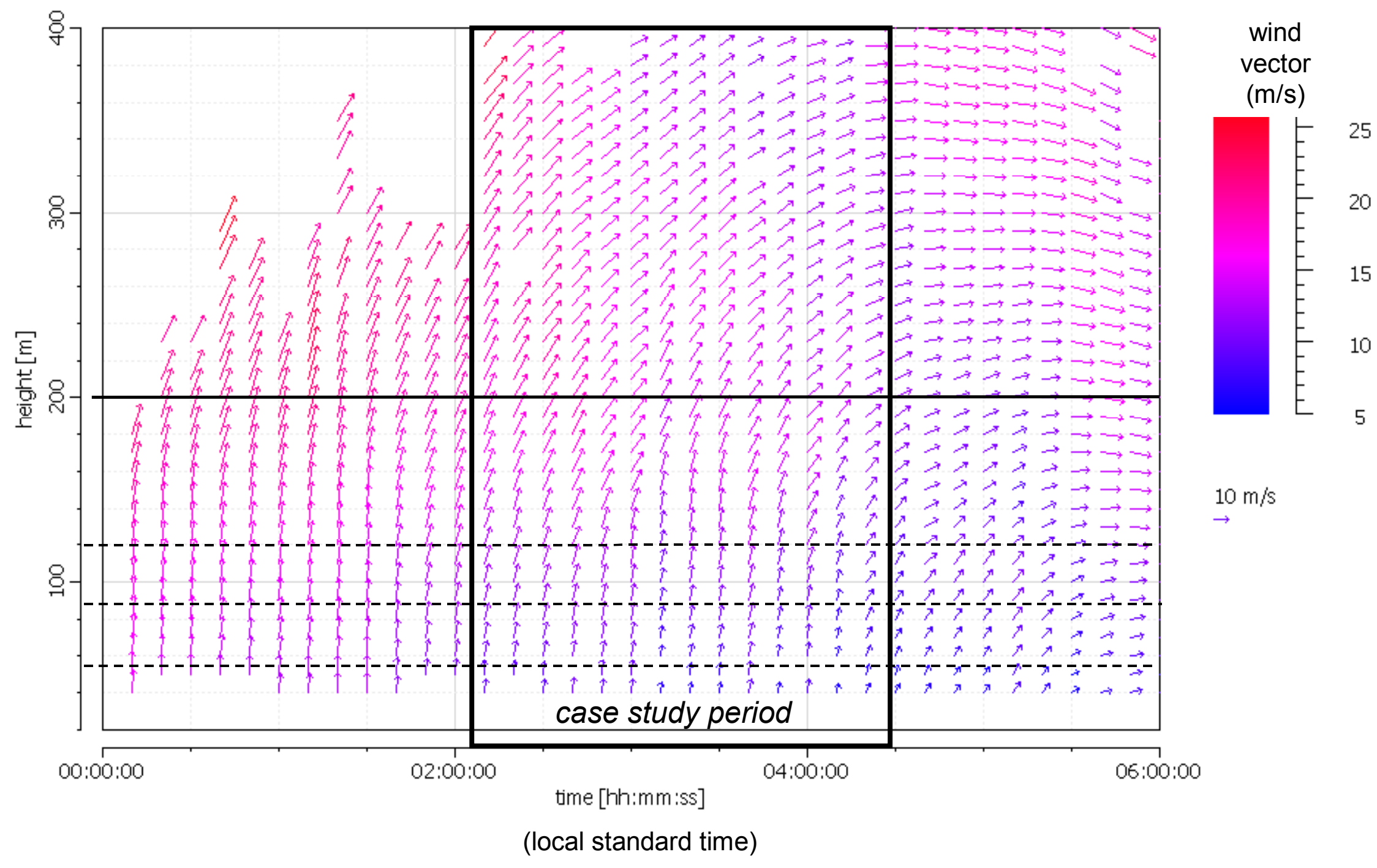

Figure 6-35. SODAR-derived 10-minute mean wind vector profiles for 0 to $06 \mathrm{~h}$ LST on 23 June 2002 $6-47$ 


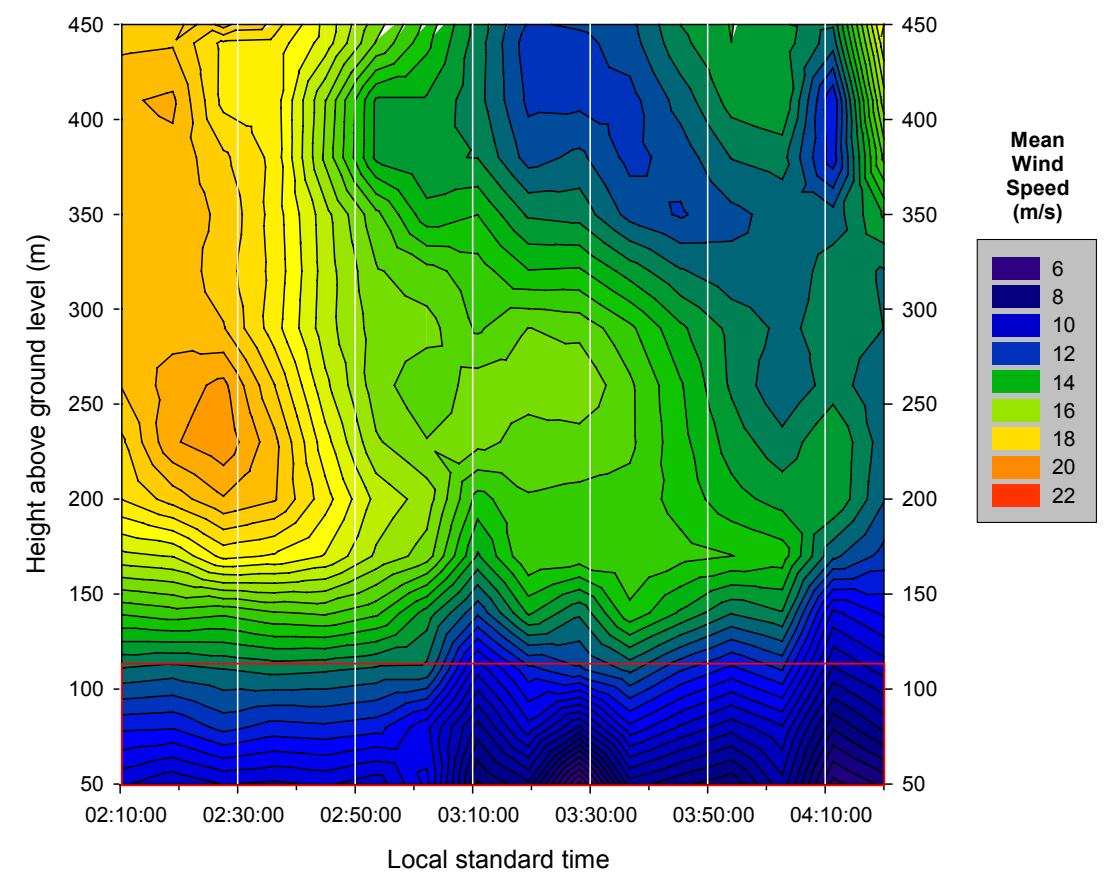

Figure 6-36. SODAR-derived mean wind speed profile contours for 23 June 2002 - AM Case

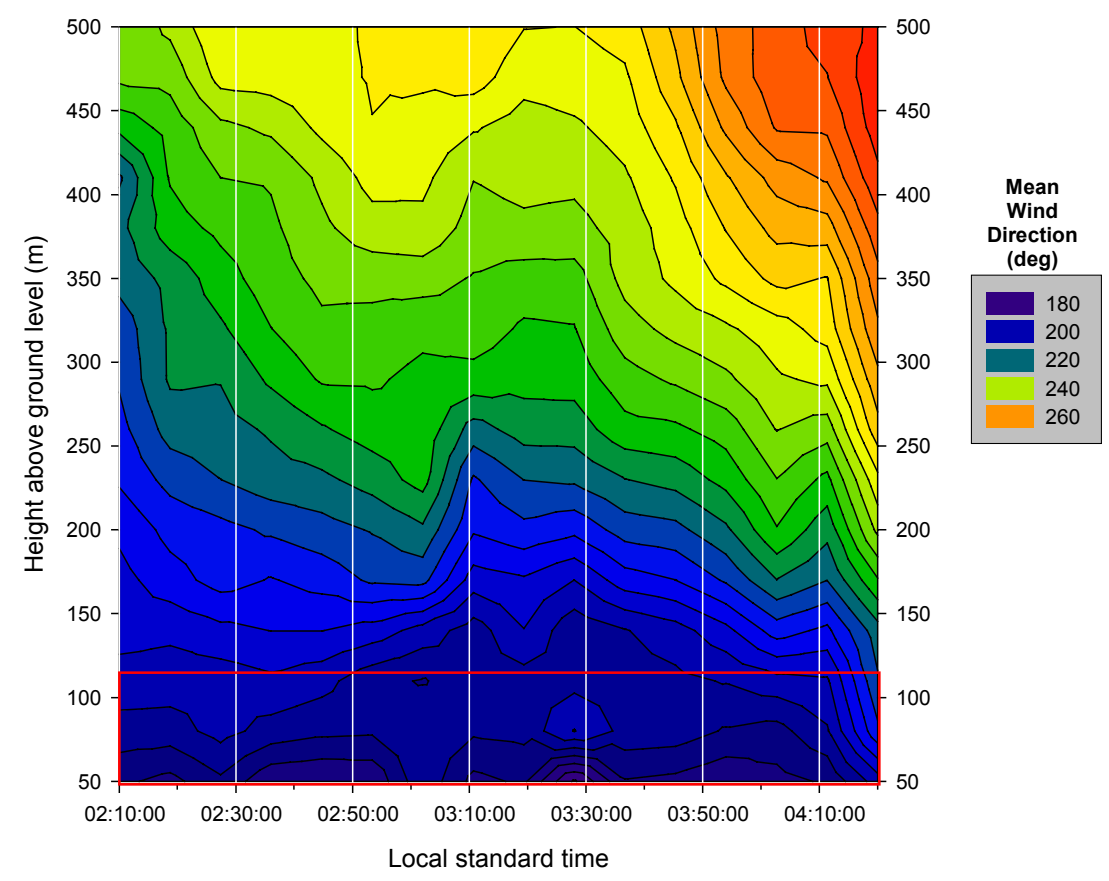

Figure 6-37. SODAR-derived mean wind direction profile contours for 23 June 2002 - AM Case 


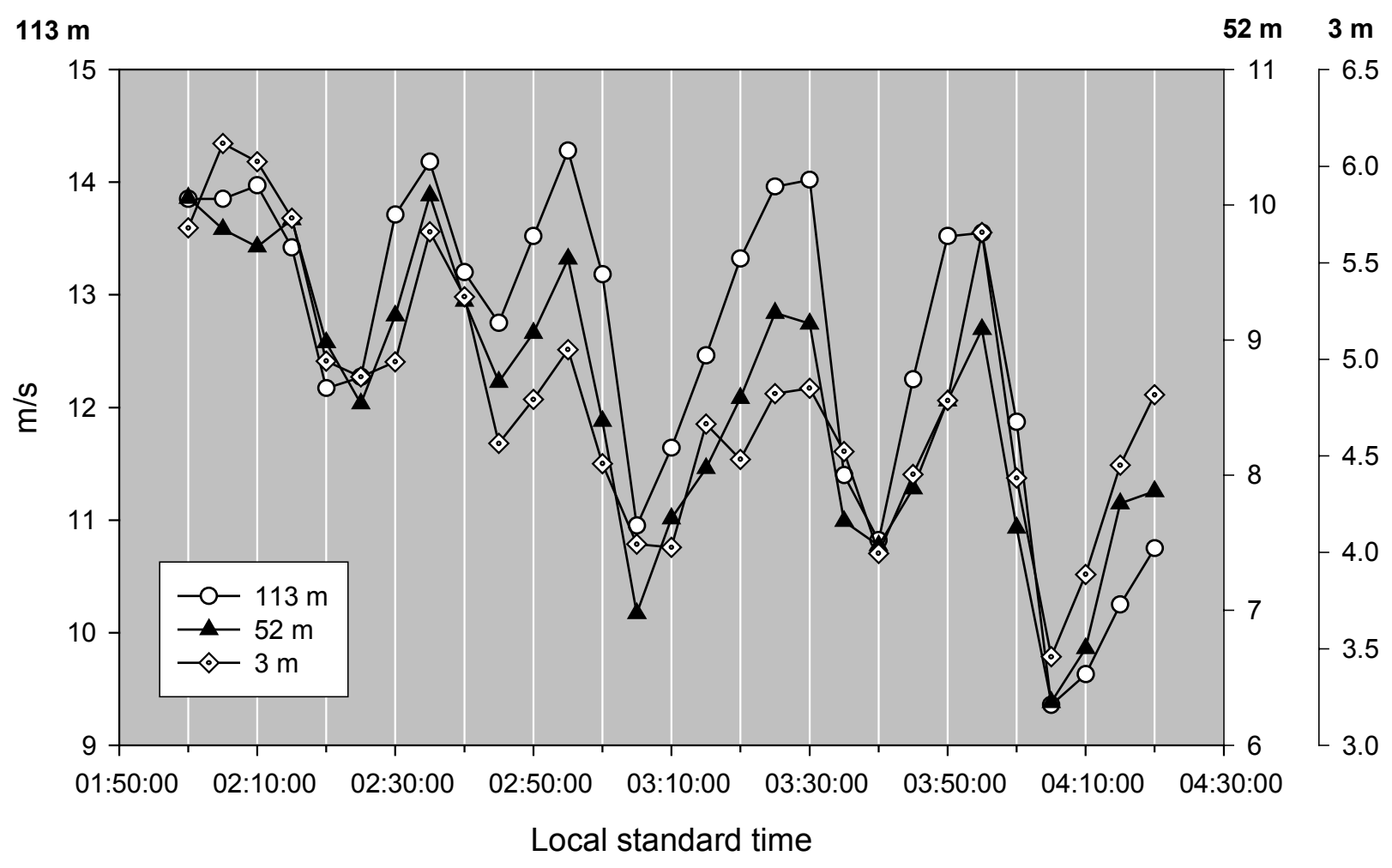

Figure 6-38. Long-period wave motions: 23 June 2002 - AM Case 

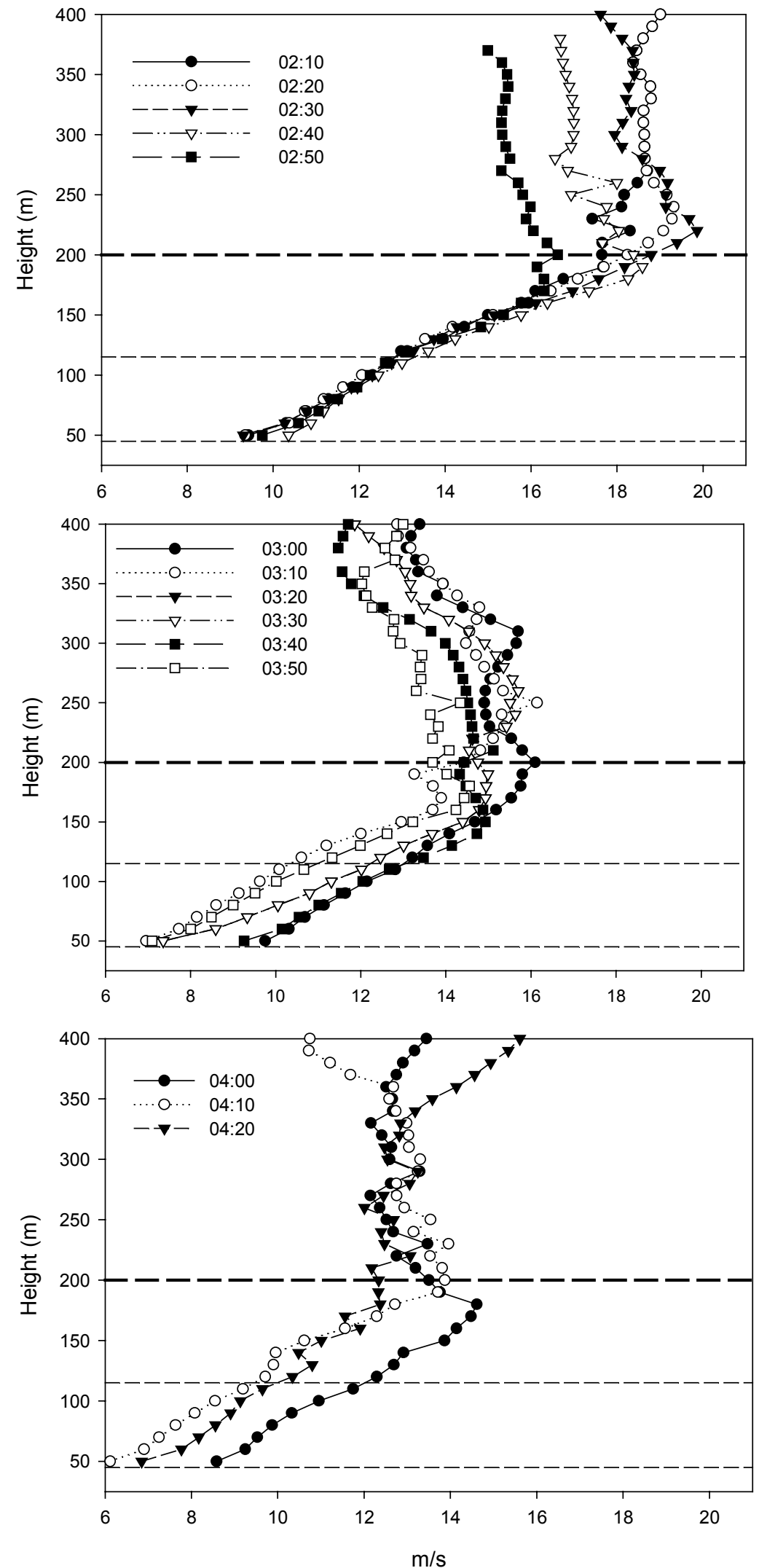

Figure 6-39. SODAR-derived wind speed profiles for 23 June 2002 - AM Case 


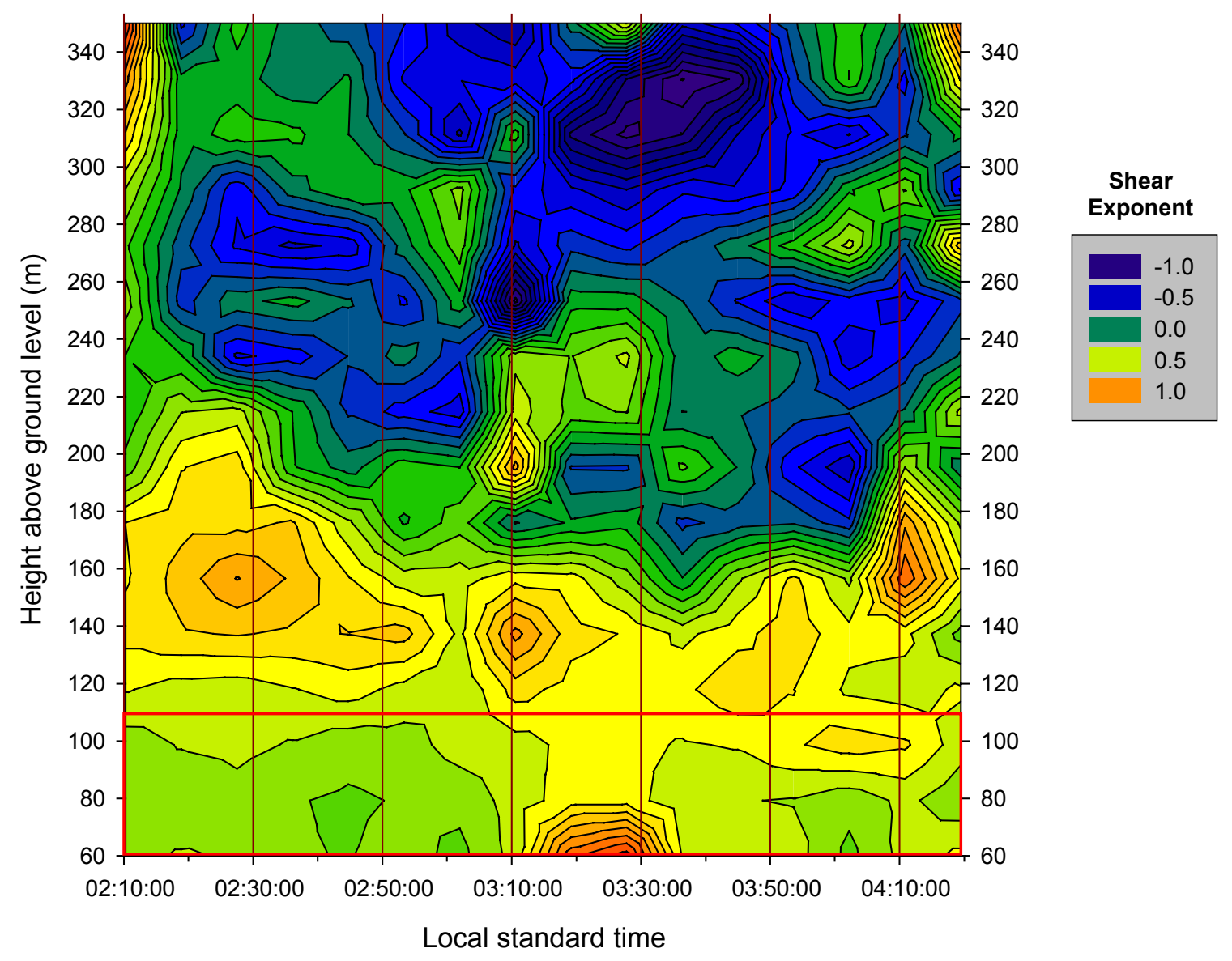

Figure 6-40. Sodar-derived vertical shear profile contours for 23 June 2002 - AM Case 


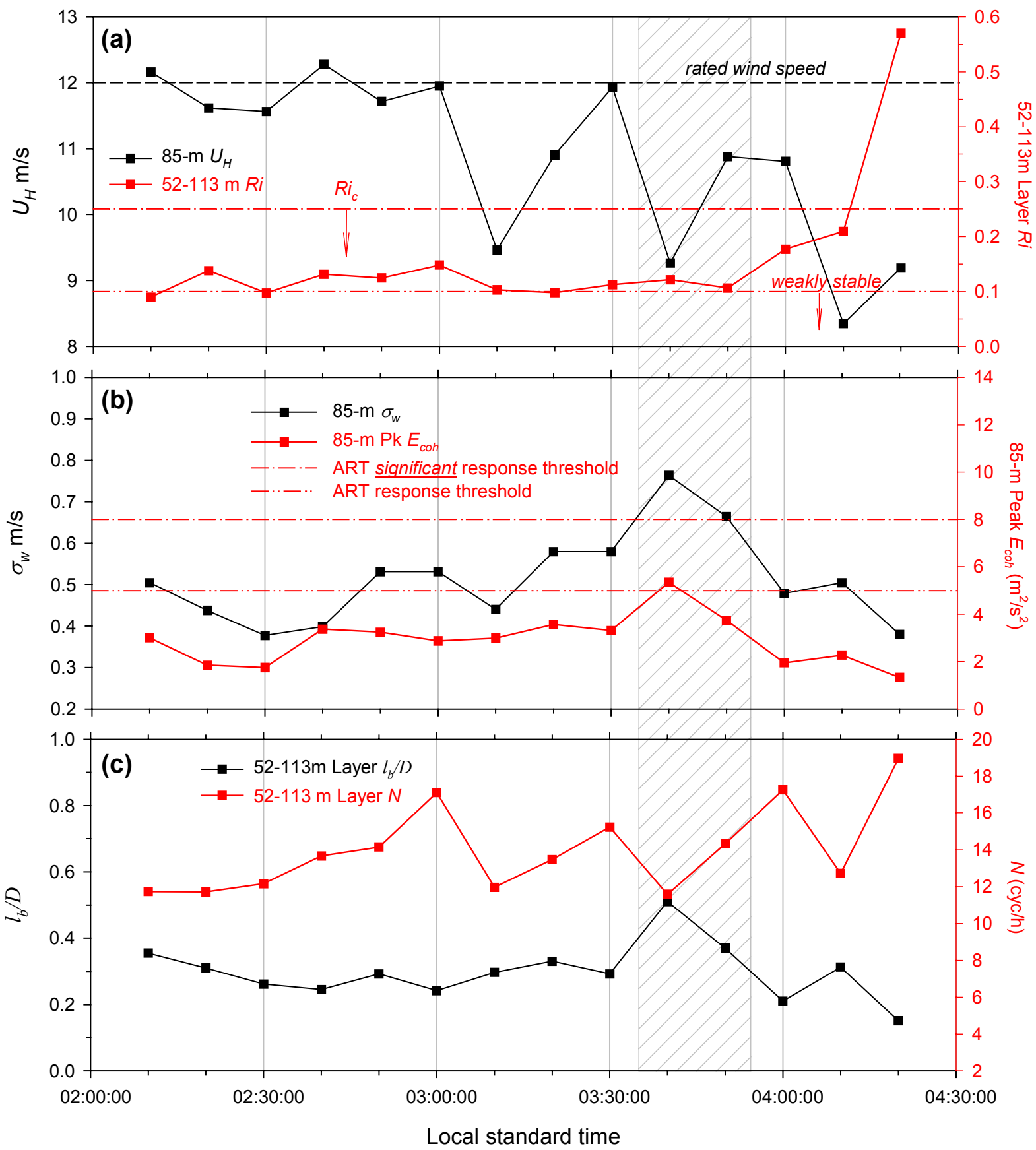

Figure 6-41. Time histories of 10-minute mean inflow parameters for 23 June 2002 - AM Case 


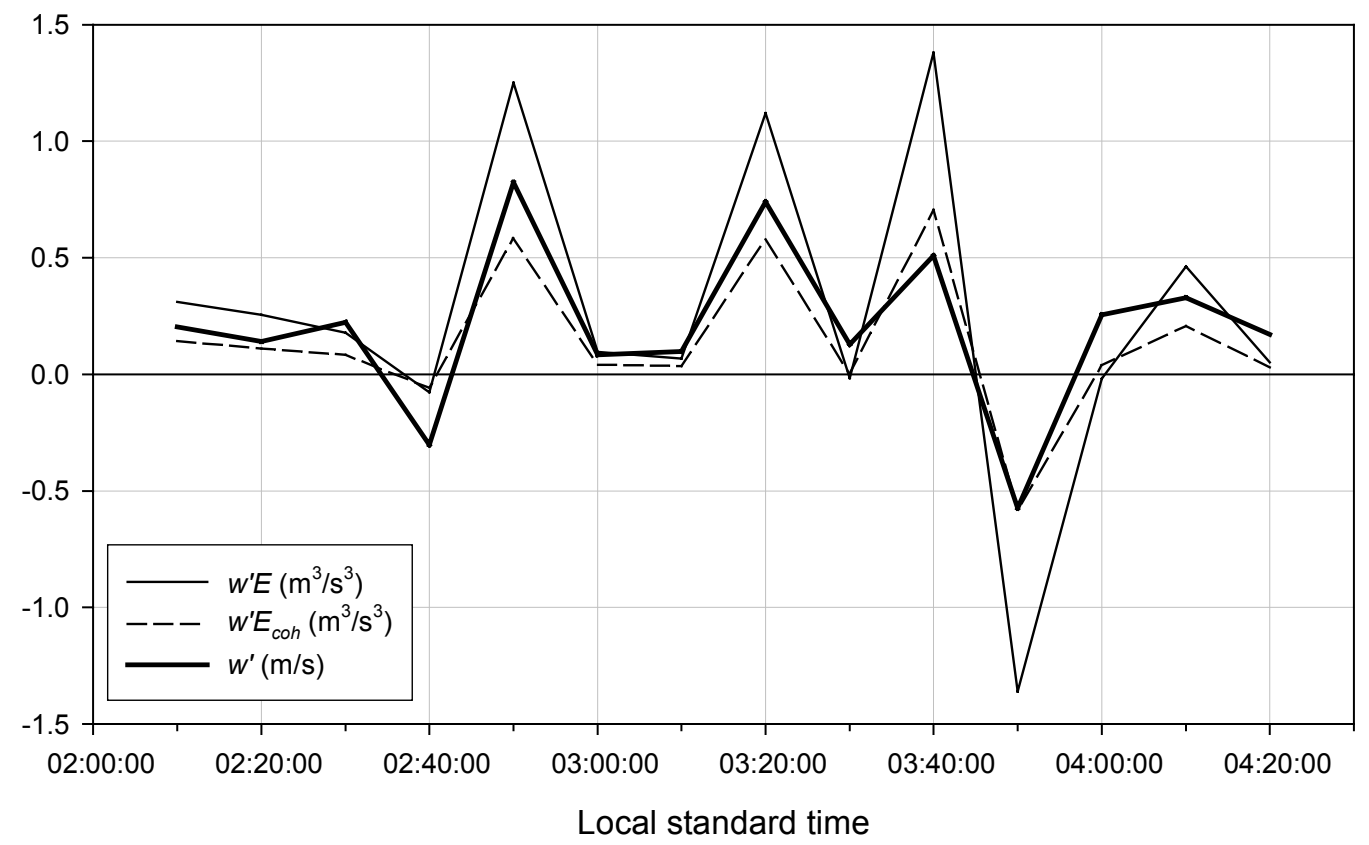

Figure 6-42. Time histories of net vertical fluxes of $E$ and $E_{c o h}$ and mean vertical velocity for 23 June 2002 - AM Case 


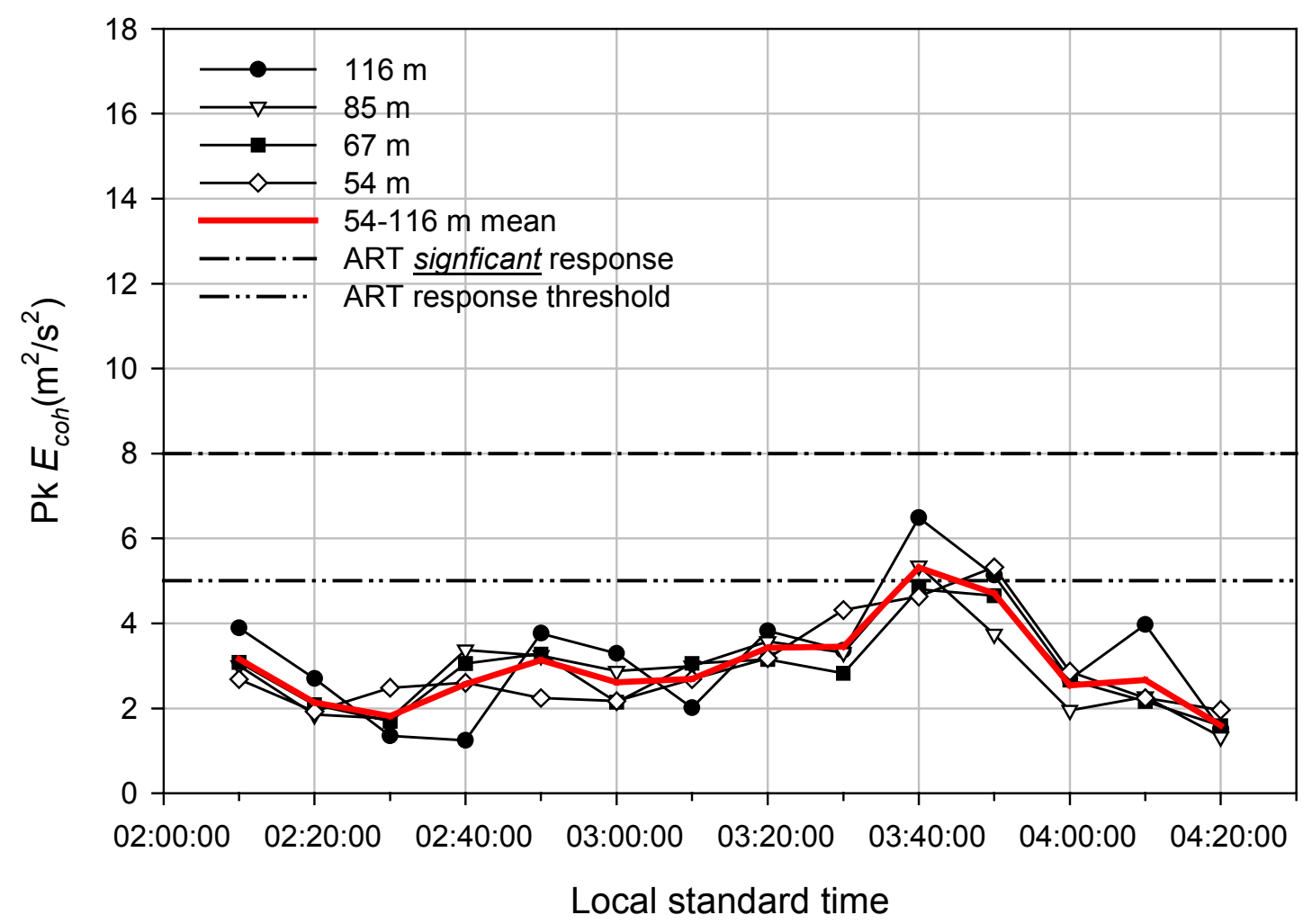

Figure 6-43. Time histories of peak coherent turbulent kinetic energy $E_{c o h}$ by height for 23 June 2002 - AM Case 


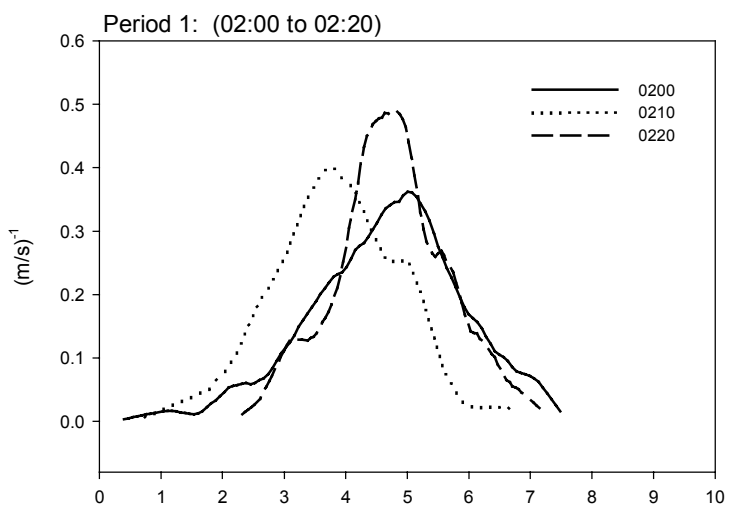

$\mathrm{m} / \mathrm{s}$

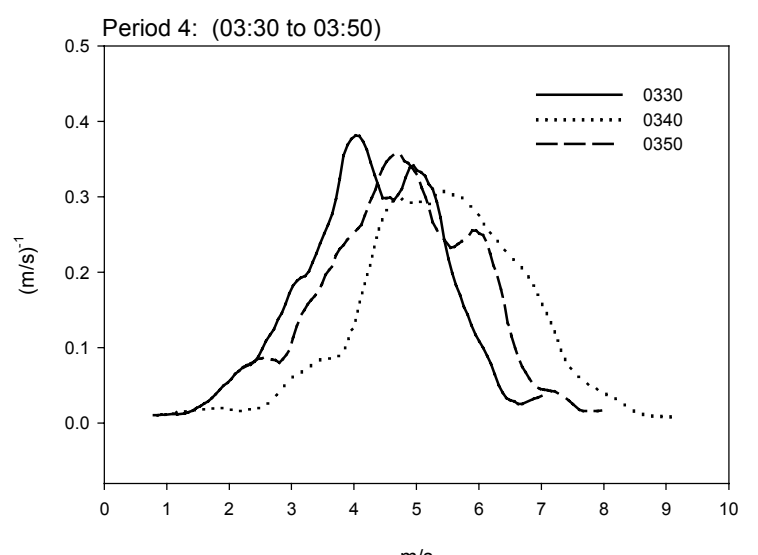

$\mathrm{m} / \mathrm{s}$

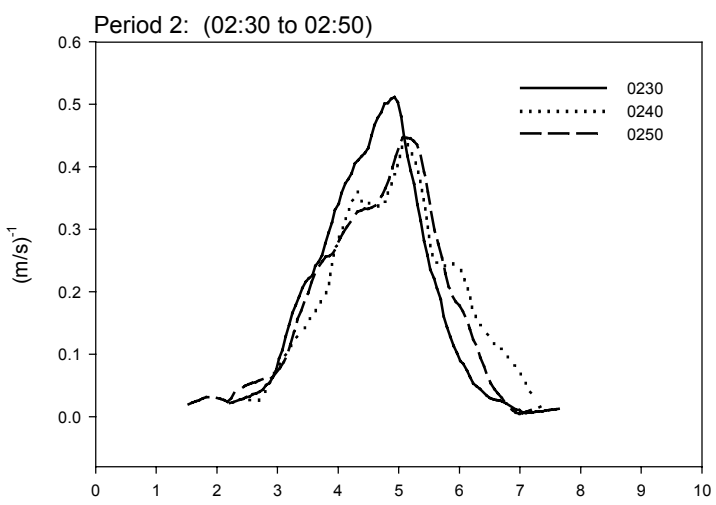

$\mathrm{m} / \mathrm{s}$

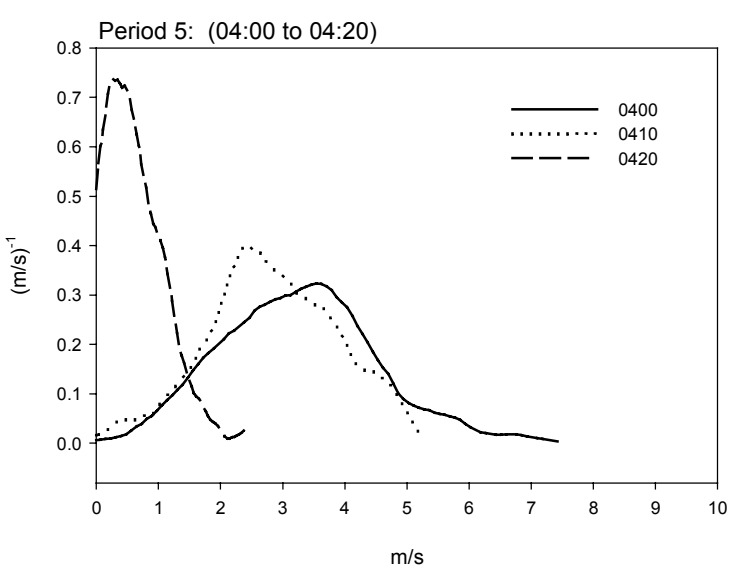

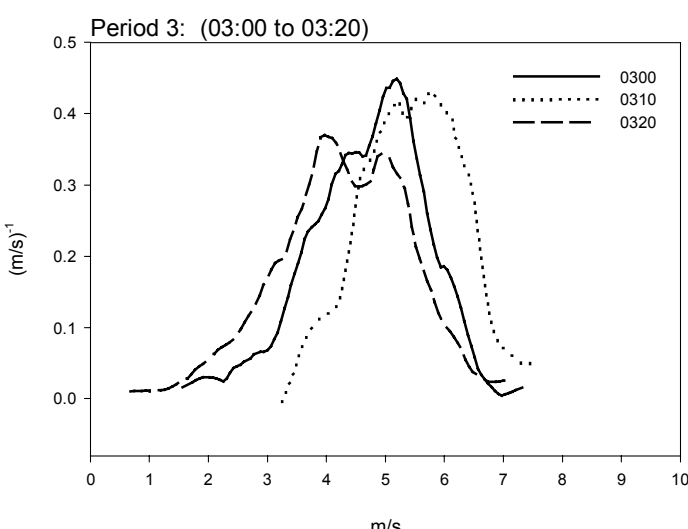

23 June 2002 Case 3-Sec Wind Speed Difference Probability Density Distributions 116-54 m Height (Full Rotor) (Scaled for $70.5 \mathrm{~m}$ rotor diameter)

Figure 6-44. Probability density distributions of 3-s wind speed differences for 23 June 2002 - AM Case 


\subsection{Using NWTC/LIST Results to Estimate Turbine Response}

We intend to use the previous case study measurements to obtain an initial estimate of the response of a turbine operating at the LLLJP Site. To do that, we will aggregate the turbulence inflow information gathered with these three case studies to ascertain how the ART turbine rotor would respond, at least statistically, if installed at that location and at the height of the future GE $1.5 \mathrm{~S}$ turbines. First, we review and summarize the salient points regarding the interrelationships between turbulence scaling variables found when analyzing the case studies in the previous section. We will next use our simple linear regression model of the ART response to predict statistical measures of the root flapwise equivalent loads $\left(L_{e q}\right)$, the maximum alternating load cycle $\left(L_{p-p}\right)$, the extreme load $\left(L_{p k}\right)$, and the extreme or peak zero-mean load $\left(\hat{L}_{p k}\right)$ using predictor values measured at the LLLJP Site on the GE Wind 120-m tower. We will then compare the predictions with the population distributions of these response parameters measured on the ART turbine at the NWTC during the LIST Program.

\subsection{Summary of Relationships Between Turbulent Structural Parameters and Turbine Response for LLLJP Case Studies}

In this section, we summarize the relationships between parameters related to the organized structure of the turbulence seen during the case studies discussed in Section 6. In particular, we are interested in parameters related to the effects of buoyancy through their influence on the level of coherent turbulence generated and the resulting turbine response. These include the buoyancy length scale, $l_{b}$ (or equivalently the $l_{b} / D$ ratio); the hub-height peak coherent turbulence kinetic energy, $E_{c o h}$; the buoyancy or Brunt-Väsällä frequency, $N$; and the gradient Richardson number, $R i$. The relationship between $l_{b}$ and peak $E_{c o h}$ and their impact on the ART turbine response is summarized for each of the Section 6 case studies in Figure 7-1. There is a very close correlation between the characteristic wave motion scale $\left(l_{b}\right)$ and peak value of $E_{c o h}$ contained with a 10-minute observed record. The figure contents also underscore the fact that significant levels of turbine response occur when $l_{b}$ approaches (or exceeds) the turbine rotor diameter (indicated by the dashed black line), which is a $l_{b} / D$ ratio of 1 or greater. These relationships are summarized by a single graph (Figure 7-2) where the peak $E_{c o h}$ associated with the 54-, 67-, 85-, and 116-m heights are plotted as functions of $l_{b}$ and $l_{b} / D$.

The influence of the rotor layer $R i$ on $l_{b}$ is summarized by the power law relationship $l_{b}=2.14 R i^{-1.15}$ plotted in Figure 7-3. This graph clearly demonstrates the impact of weakly stable conditions within the vertical layer occupied by the turbine rotor disk when the $l_{b}$ approaches the diameter of the turbine rotor. The buoyancy scale, in turn, then influences the peak $E_{c o h}$ value and the turbine response (Figure 7-2). The direct relationships between peak $E_{c o h}$ and both $R i$ and $N$ are plotted in Figure 7-4. Figure 7-4a demonstrates that peak $E_{c o h}$ is very sensitive to both $R i$ and $N$, with the highest levels of $E_{c o h}$ occurring with the smallest values of $N$. The observed relationship between peak $E_{c o h}$ and $N$ can be reasonably described by

$$
P k E_{c o h}=-5.533+15.562 N^{-0.655} .
$$

As discussed in Section 2, $N$ is a very important parameter in scaling atmospheric wave motions through its relationship between the vertical shear and buoyancy. The relationship expressed by 
(7-1) ties together levels of organized, coherent turbulence in the stably stratified flows present beneath nocturnal low-level jets, as seen at the LLLJP Site.

\subsection{Estimating Turbine Response at the LLLJP Site}

We will now employ our simple linear regression response model of the ART turbine developed in Section 3 to estimate the impact of the turbulence characteristics measured in the three case studies on the turbine statistical response. In other words, we would like to "place" the ART rotor in the inflow conditions seen at the height of a future GE 1.5S rotor installed at the LLLJP Site and examine the reaction.

\subsubsection{NWTC/LIST and LLLJP Case Studies Predictor Variable Distributions}

First, we present the observed (sample population) cumulative probability distributions of the four response variables derived from the root flapwise moment: the equivalent load $L_{e q}$, the maximum alternating load $L_{p-p}$, the extreme load $L_{p k}$, and the peak zero-mean load $\hat{L}_{p k}$ in Figure 7-5 separated into unstable $(0<R i$ shown by the red dashed line) and stable $(R i>0$ shown by the blue solid line) turbine layer conditions. The ART $L_{e q}$ distributions plotted in Figure 7-5a show that the highest equivalent (fatigue) loads are associated with stable flow conditions. Similar situations exist in the distributions of the remainder of the response variables in Figures 7-5b, 7$5 \mathrm{c}$, and $7-5 \mathrm{~d}$, but they are not as marked.

In Figures 7-6 and 7-7, we compare the cumulative probability distributions of the primary predictor variables at hub-height $U_{H}, \sigma_{w}, l_{b}$ (and $l_{b} / D$ ) and the appropriate Ri for the stable NWTC ART measurements and the LLLJP case studies. We use the turbine layer $R i$ for the ART data and the rotor layer $(52-113 \mathrm{~m})$ for the LLLJP. Though it was not included as predictor variable in the present regression model because of its high degree of colinearity with $\sigma_{w}$, we have also included the cumulative probability distribution of the peak value of $E_{c o h}$ based on our previous discussion in Section 7.1.

Figure 7-6a shows that the hub mean wind speeds were higher for the LLLJP case studies than for the LIST data. This is because of the following: (1) the hub height of the ART turbine was $37 \mathrm{~m}$ versus $80 \mathrm{~m}$ for the GE $1.5 \mathrm{~S}$ turbine, and (2) the ART peak power control algorithm often shut the machine down in higher wind speeds. The distributions of $\sigma_{w}$ for only stable flows depicted in Figure 7-6b reflect the nature of the two sites. The NWTC/LIST data was collected immediately downwind of a major mountain range (the Rockies) where strong downslope winds were a common occurrence during the period of NWTC/LIST data collection. The LLLJP case study data, while collected at a higher elevation, reflects conditions associated with a Great Plains site (i.e., flat, relatively homogeneous terrain). The processes that influence the $\sigma_{w}$ distribution at the LLLJP Site depend largely on shear flow instabilities that generate atmospheric wave motions and much less on any local downslope winds that may be present as a result of frontal passages and thunderstorms.

The much stronger nocturnal downslope flows experienced at the NWTC during the LIST experiment, while often producing extended periods of net downward vertical motions, are also responsible for creating shear flow instabilities and resulting wave motions [25, 26, 27, 28]. What has been said of the $\sigma_{w}$ distributions also applies to the probability distributions of peak $E_{c o h}$ in Figure 7-6d. It is likely that when the entire LLLJP data set is processed and analyzed the 
high value tails will move closer to those from the NWTC. Finally, the NWTC/LIST and LLLJP probability distributions of the buoyancy length scale $l_{b}$ and $l_{b} / D$ shown in Figure 7-7 are quite similar even though the latter is quite sparse. This similarity suggests that a similar range of wave motions occurs at both locations with comparable frequencies (at least for the small LLLJP sample). Again, when the entire available LLLJP dataset is processed and analyzed, we believe the relationship indicated in Figure 7-7 will become clearer.

\subsubsection{Comparison of Predicted ART Response at LLLJP Site with NWTC}

We now compare the predicted statistical response of the ART turbine placed at the LLLJP with its performance at the NWTC during the LIST Program. We used our simple linear regression model and the limited range of predictor values available from the three case studies discussed in Section 6 to predict the statistical behavior of the root flapwise bending equivalent loads $\left(L_{e q}\right)$, the maximum peak-to-peak load amplitude $\left(L_{p-p}\right)$, the extreme load $\left(L_{p k}\right)$, and the peak zero-mean load $\left(\hat{L}_{p k}\right)$ in the LLLJP inflow environment. The cumulative probability distributions for each of the response variables measured on the ART turbine as part of the NWTC/LIST Program with the $1^{\text {st }}, 5^{\text {th }}, 10^{\text {th }}, 20^{\text {th }}$, and $50^{\text {th }}$ percentiles of the predicted values for the LLLJP Site are indicated in Figure 7-8.

With the exception of the extreme flapwise loads $L_{p k}$ in Figure 7-8c, the remainder of the LLLJP predicted percentiles are less than those actually observed at the NWTC. The predications of the equivalent loads $L_{e q}$ and maximum alternating load cycles $L_{p-p}$ are both within one standard deviation of the NWTC in the $5^{\text {th }}, 10^{\text {th }}$, and $20^{\text {th }}$ percentiles and perhaps within two standard deviations in the $1^{\text {st }}$ and $50^{\text {th }}$ percentiles (Figures $7-8 \mathrm{a}$ and $7-8 \mathrm{~b}$ ). The largest deviation is with the predicted peak zero-mean loads $\hat{L}_{p k}$ (Figure 7-8d). This is consistent with the performance of the linear regression model summarized in Table 3-5, where the coefficient of multiple regression $\left(r^{2}\right)$ was 0.573 compared to the others that had $r^{2}$ values of 0.699 and higher. We believe that including additional or perhaps substituting other predictor variables, such as the hub friction velocity $u_{*}$ or $\overline{u^{\prime} w^{\prime}}$ (shear stress), in the regression will likely improve the performance of this prediction and perhaps the others as well.

The closeness of the predicted and observed percentiles of the equivalent loads $L_{e q}$ and maximum alternating load cycles $L_{p-p}$ is important because of the relationship of the former $\left(L_{e q}\right)$ in assessing fatigue damage [33]. The load-limiting performance of the ART peak power control algorithm probably has considerable influence on the good prediction of the extreme loads $L_{p k}$. The change in slope of the cumulative probability at the $50^{\text {th }}$ percentile value of about $520 \mathrm{kNm}$ corresponds to the level at which active load limiting typically commenced. This is demonstrated in Figure 7-5c, where the load distributions for both unstable and stable flows are plotted with very similar results. Thus, we believe it is the load-limiting action of the control system that dominates the extreme load cumulative distribution, making it more deterministic and easier for the regression to explain. The over prediction of high loads at the $1^{\text {st }}$ percentile level probably reflects the inability of the current regression model using only $\overline{U_{H}}$ and $\sigma_{w}$ as predictors. Perhaps including another appropriate independent variable would improve the correlation and reduce the level of the unexplained variance.

The reasonable performance of this simplistic linear regression response model using only a few predictors is encouraging, with $70 \%$ or more of the observed variance being explained on three 
of the four response variables. It also indicates that the independent variables being used as predictors do have a significant influence on at least the root flapwise loads. According to Table $3-5, \sigma_{w}$ probably has the most influence, followed by $R i$ and $\overline{U_{H}}$. These results also indicate that we can use the application of the NWTC/LIST results using the ART turbine as a sensor to assess, at least at a first cut, for potential operational impacts on the turbines to be installed at the LLLJP Site. 

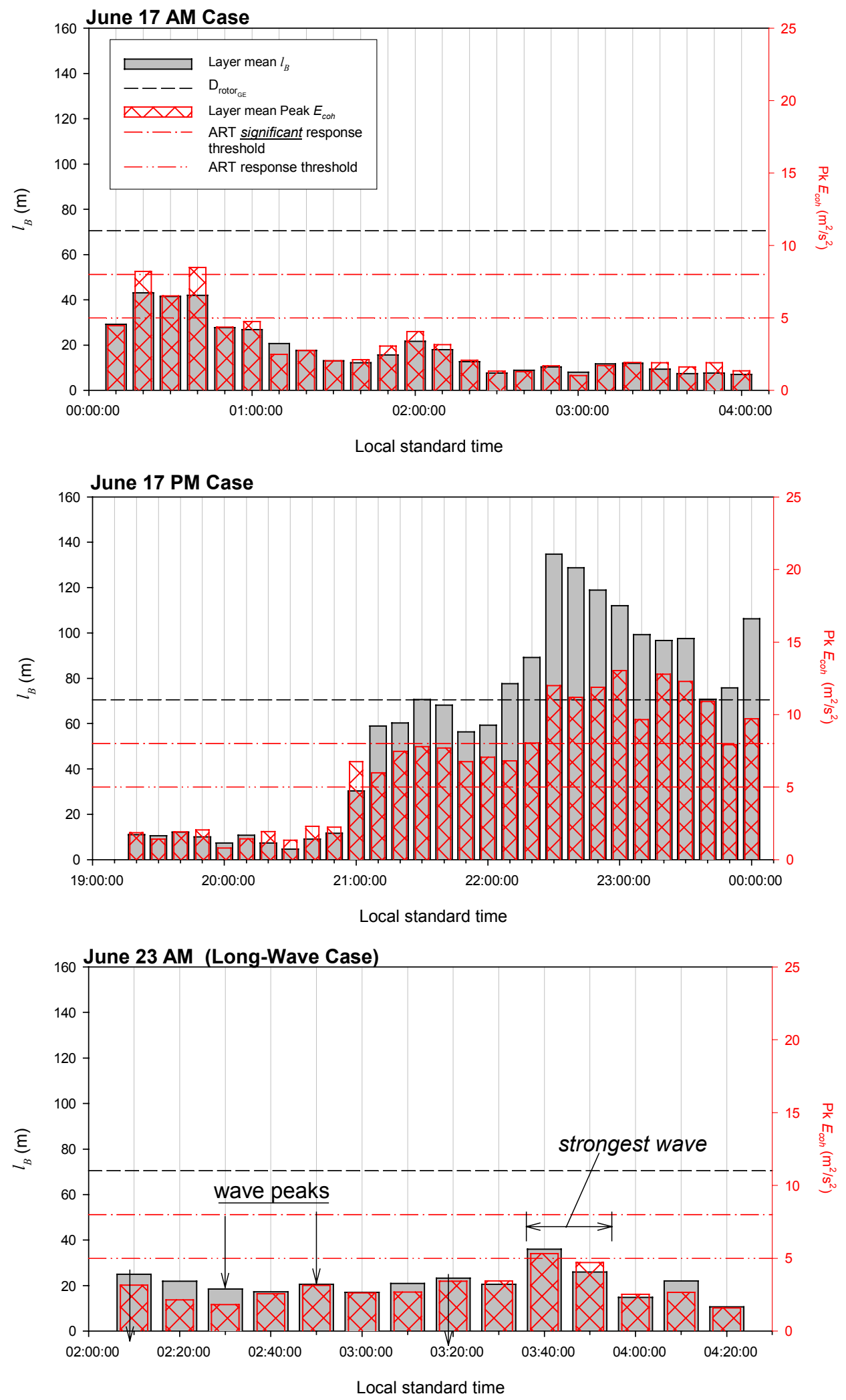

Figure 7-1. Summary of time histories of rotor layer mean $I_{b}$ and hub peak $E_{c o h}$ for the three case studies 

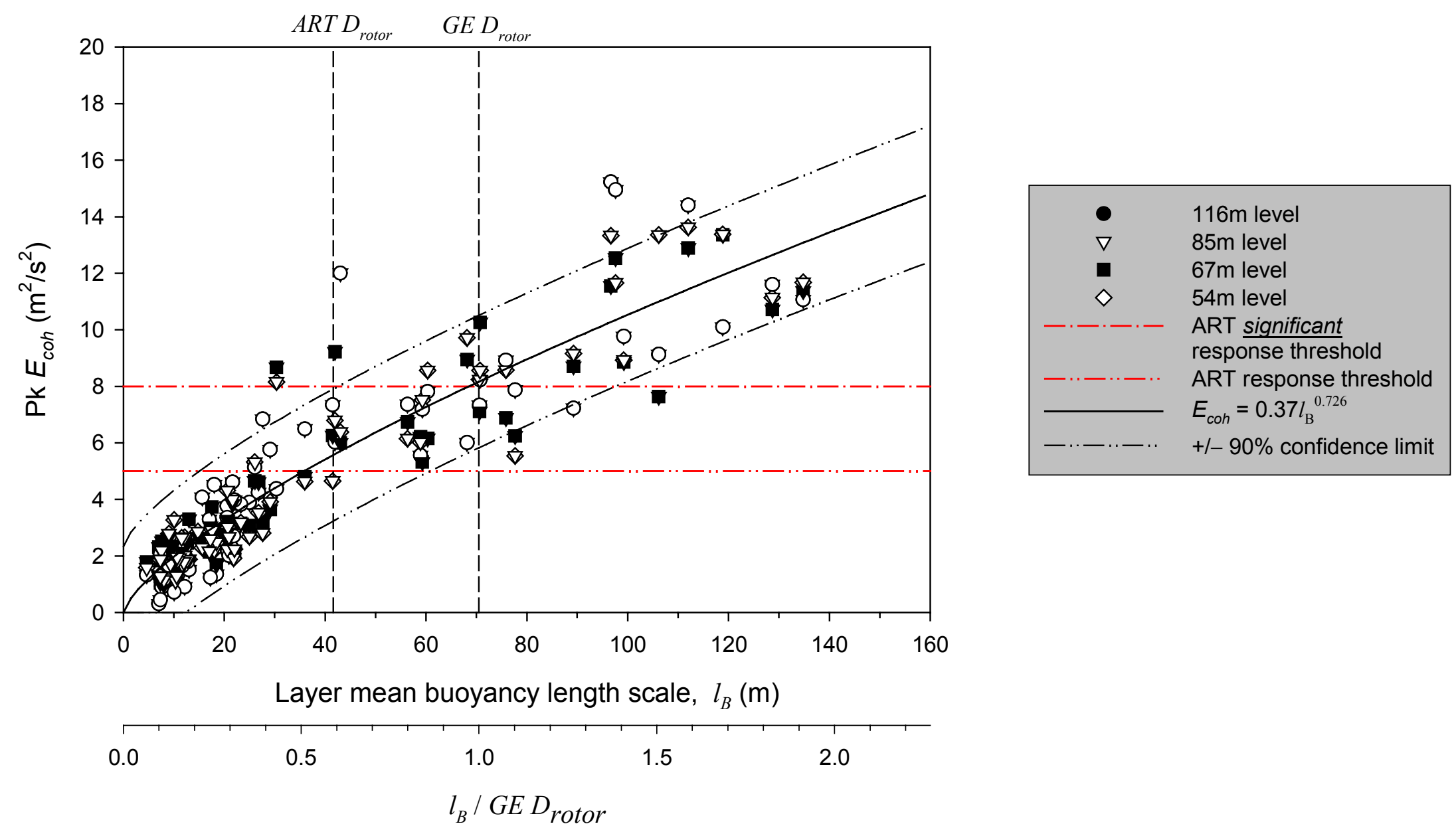

Figure 7-2. Observed variation of peak $E_{c o h}$ as a function of $I_{b}$ and $I_{b} / D$ for the three cases studies 


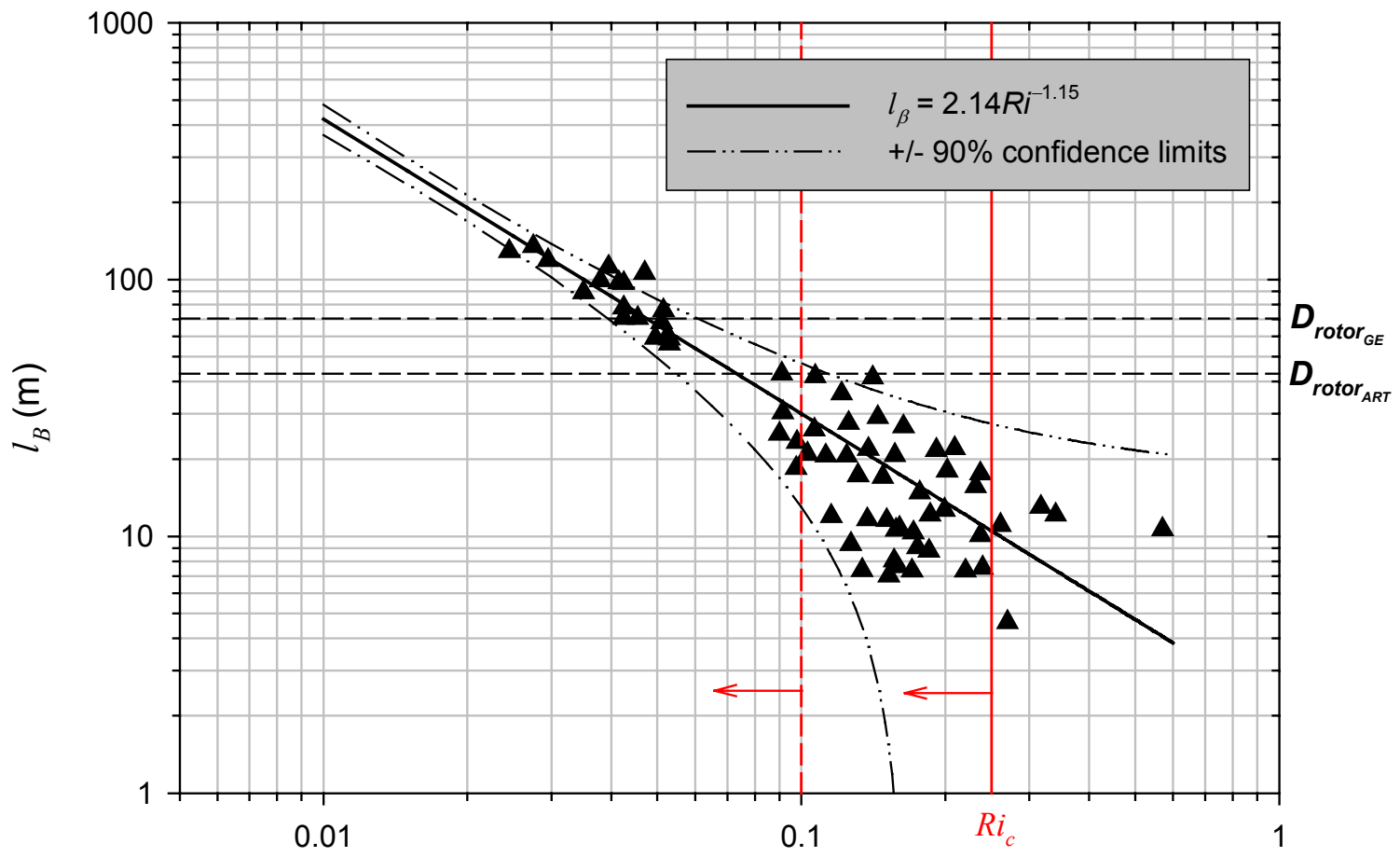

52-113m layer $R i$

Figure 7-3. Observed variation of the 52- to 113-m layer mean $I_{b}$ as a function of $R i$ for the three case studies 
(a)

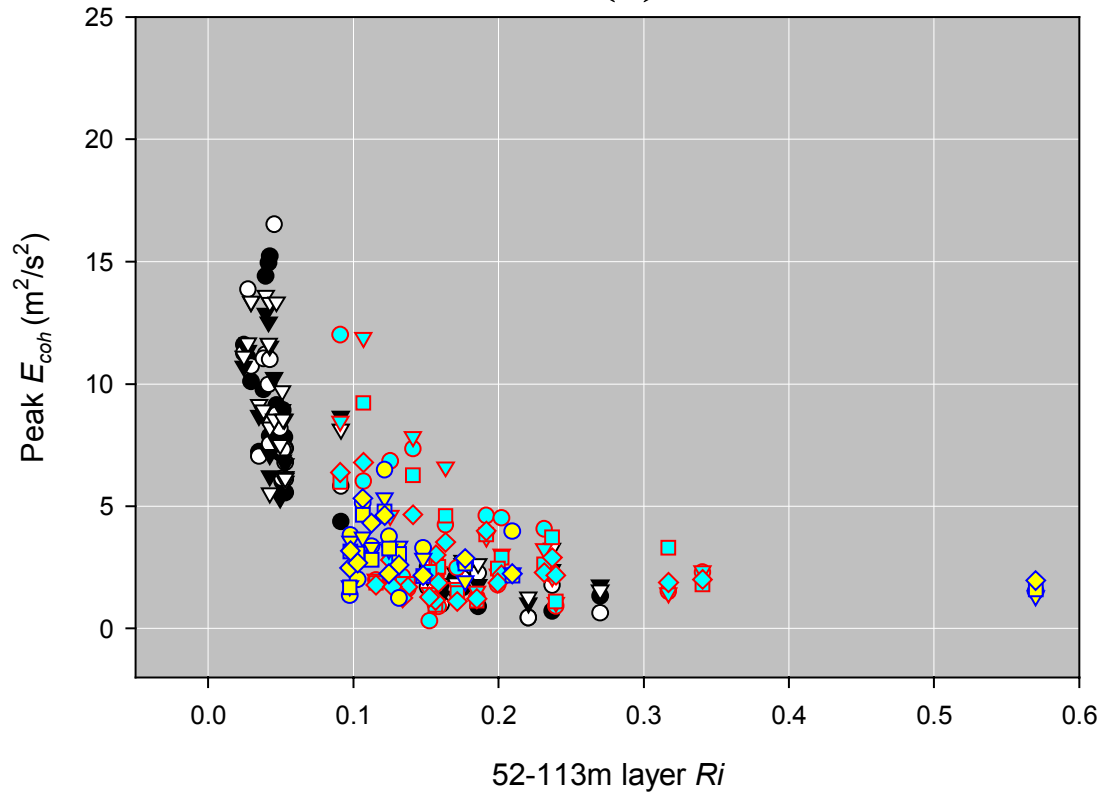

(b)

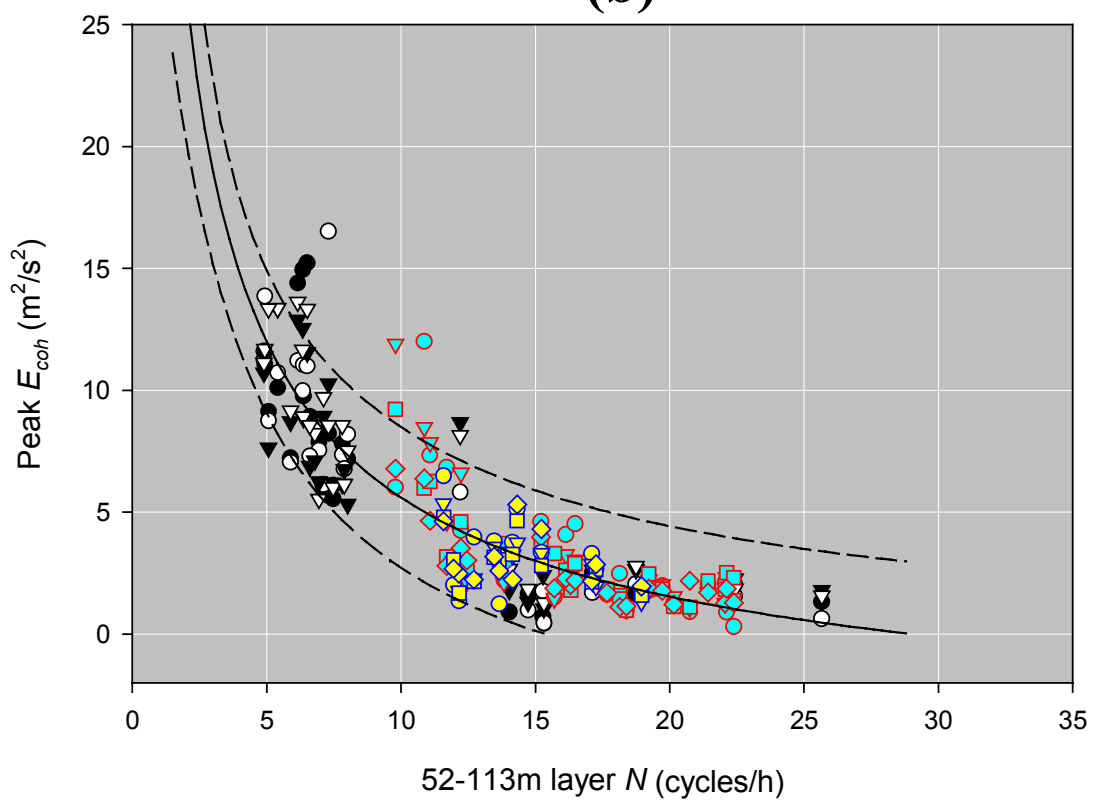

- June 17 PM - $116 m$ June 17 PM - 85m June $17 P M-85 m$
June 17 PM $-67 \mathrm{~m}$ June 17 PM - $54 \mathrm{~m}$ June 17 AM - $116 m$ June 17 AM - $85 \mathrm{~m}$ June 17 AM - $67 \mathrm{~m}$ June 17 AM - $54 m$ June $23-116 \mathrm{~m}$ June $23-85 m$ June $23-67 m$ June 23 - $54 \mathrm{~m}$ $\mathrm{Pk} E_{c o h}=-5.533+15.562 N^{-0.655}$ $+/-90 \%$ prediction limit

Figure 7-4. Observed variation of the peak $E_{\text {coh }}$ of the 52-113-m layer as a function of $R i$ and $N$ for three case studies as a function of 52- to 113-m layer: (a) $R i$, (b) buoyancy frequency $N$ 
(a)

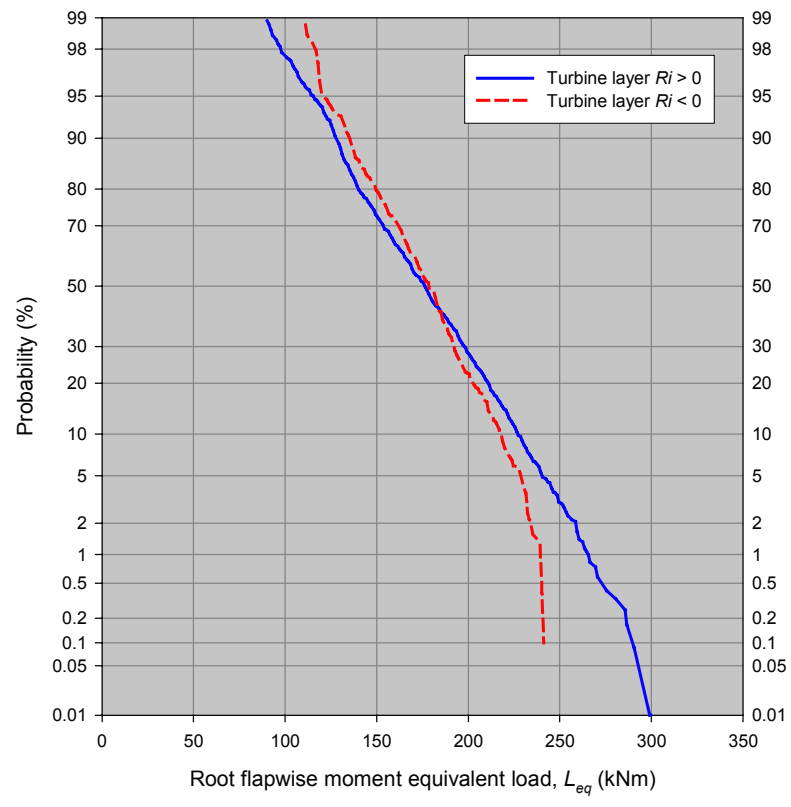

(c)

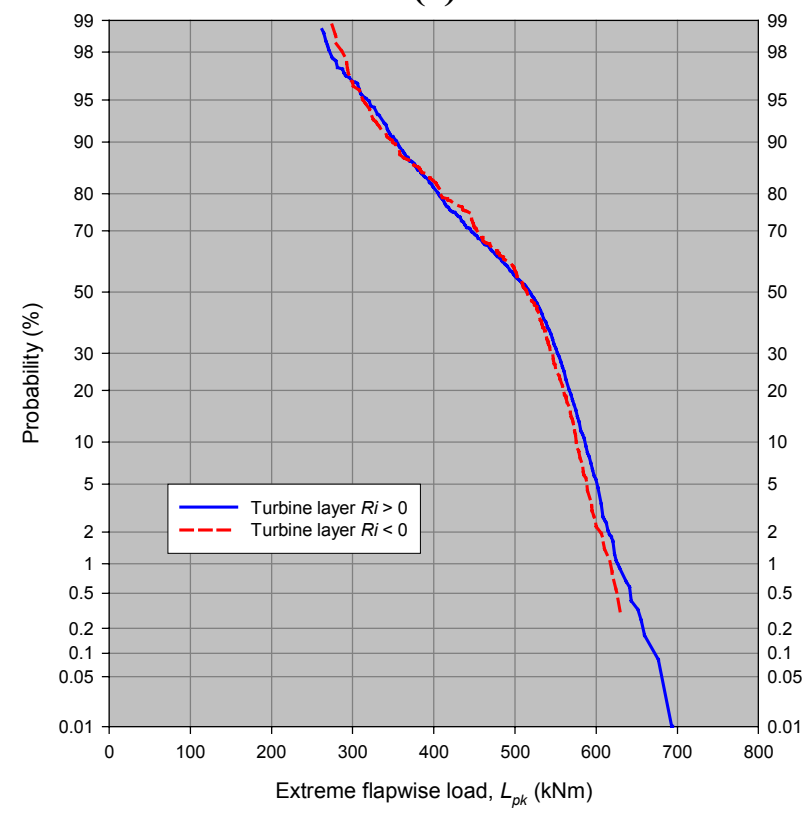

(b)

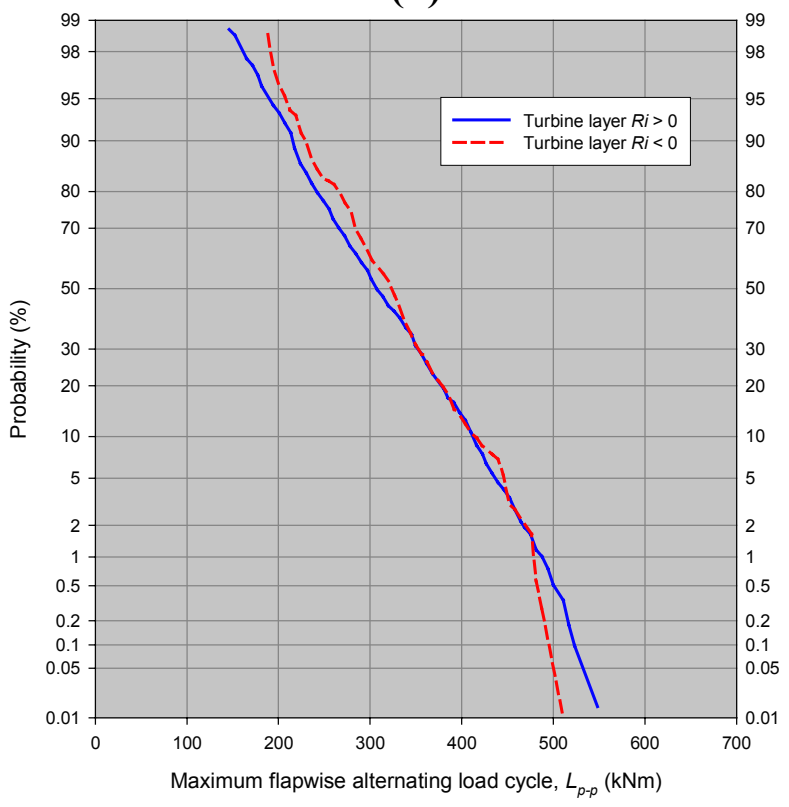

(d)

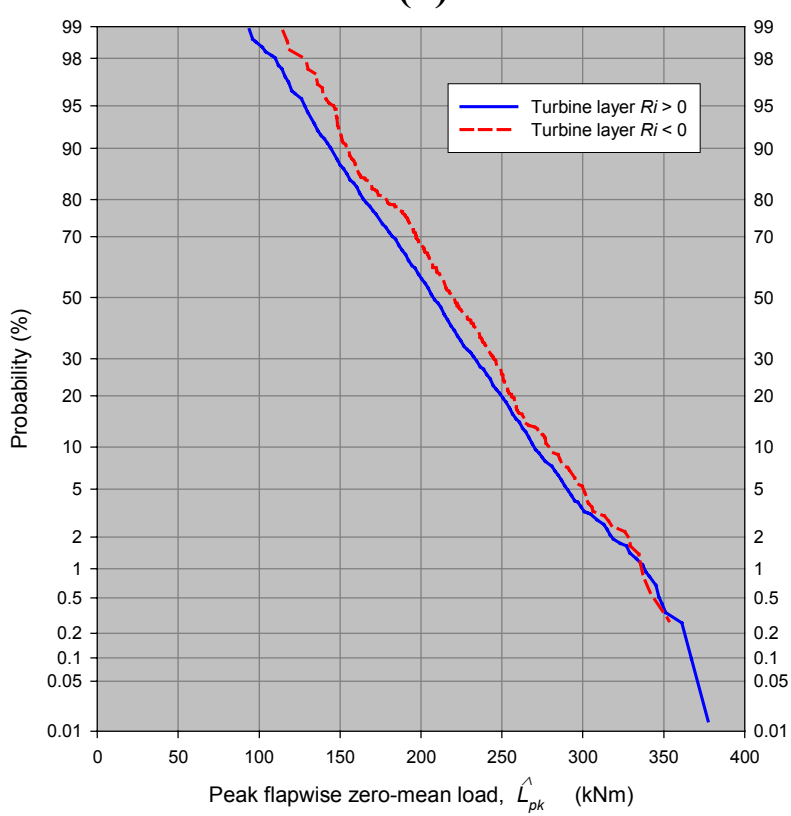

Figure 7-5. Sample population cumulative probability distributions of NWTC/LIST ART turbine root flapwise (a) equivalent load $L_{\text {eq }}$, (b) maximum alternating load cycle $L_{p p}$, (c) extreme load $L_{p k}$, and (d) peak zero-mean load $\hat{L}_{p k}$ for unstable and stable inflows 
(a)

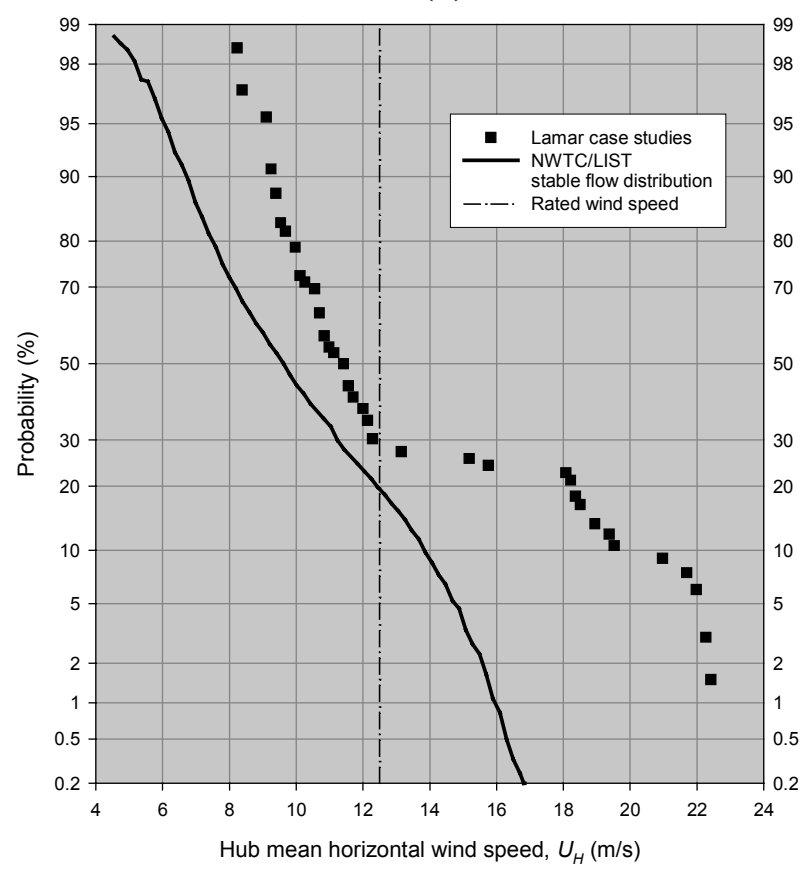

(c)

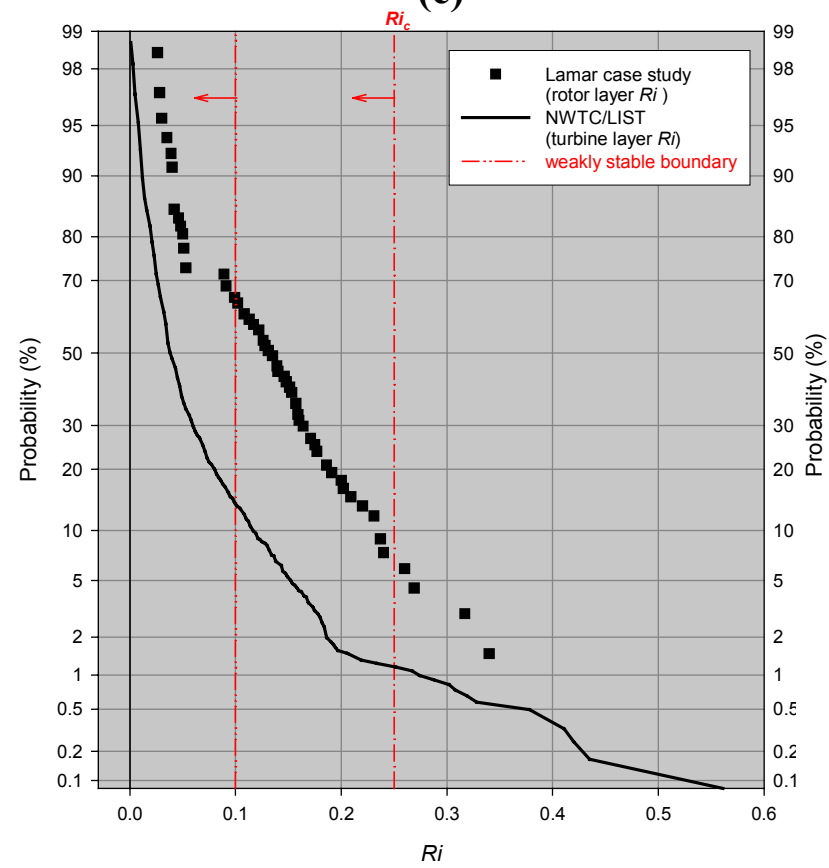

(b)

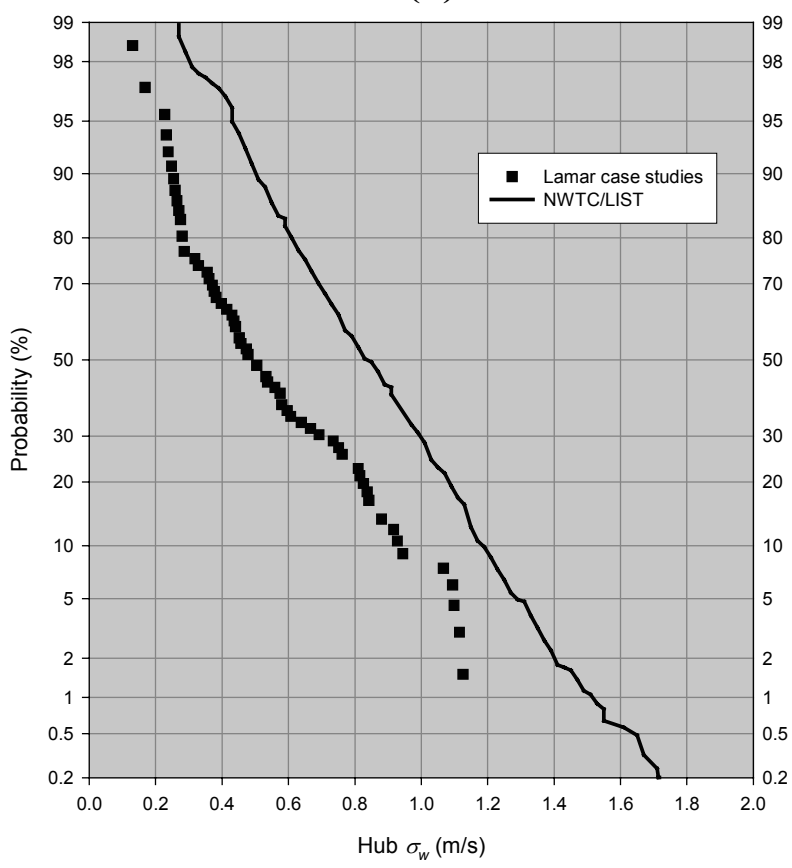

(d)

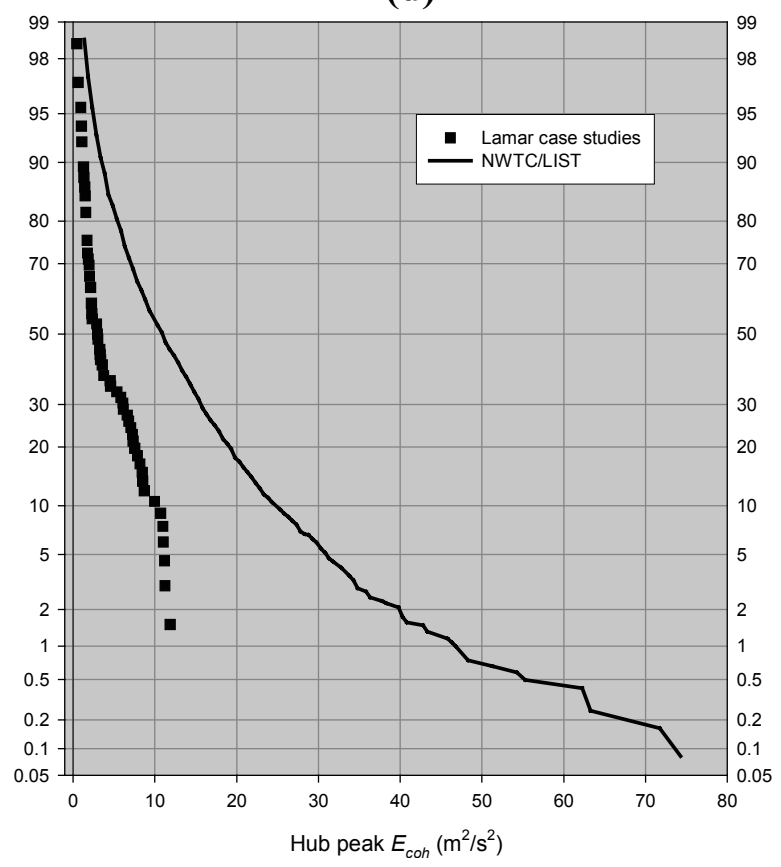

Figure 7-6. Cumulative probability distributions of stable NWTC/LIST and three LLLJP case studies hub mean (a) $U_{H}$, (b) $\sigma_{w}$, (c) $R i$, and (d) peak $E_{c o h}$ for stable flows 


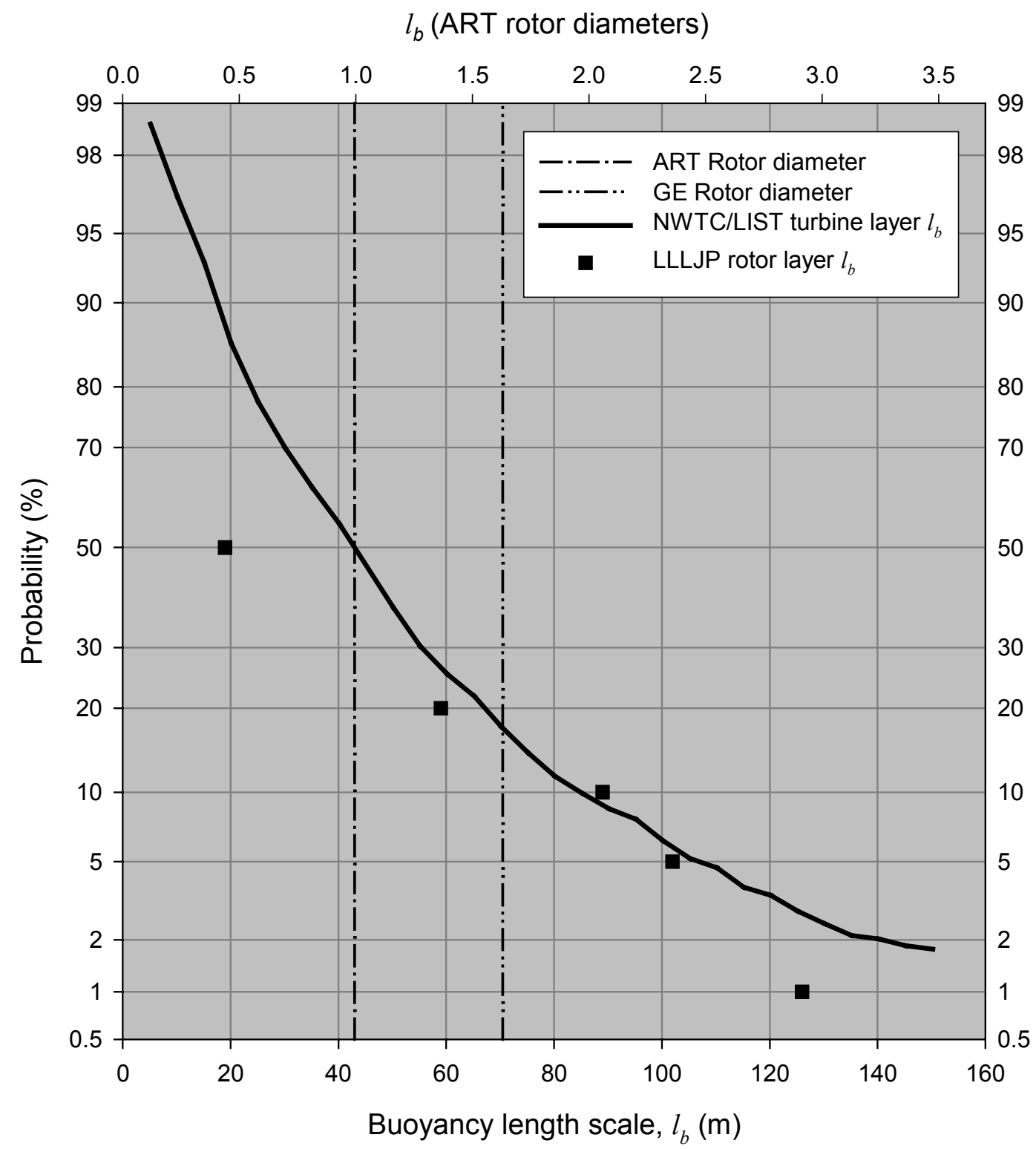

Figure 7-7. Probability distributions of NWTC/LIST and three LLLJP case studies $I_{b}$ 
(a)

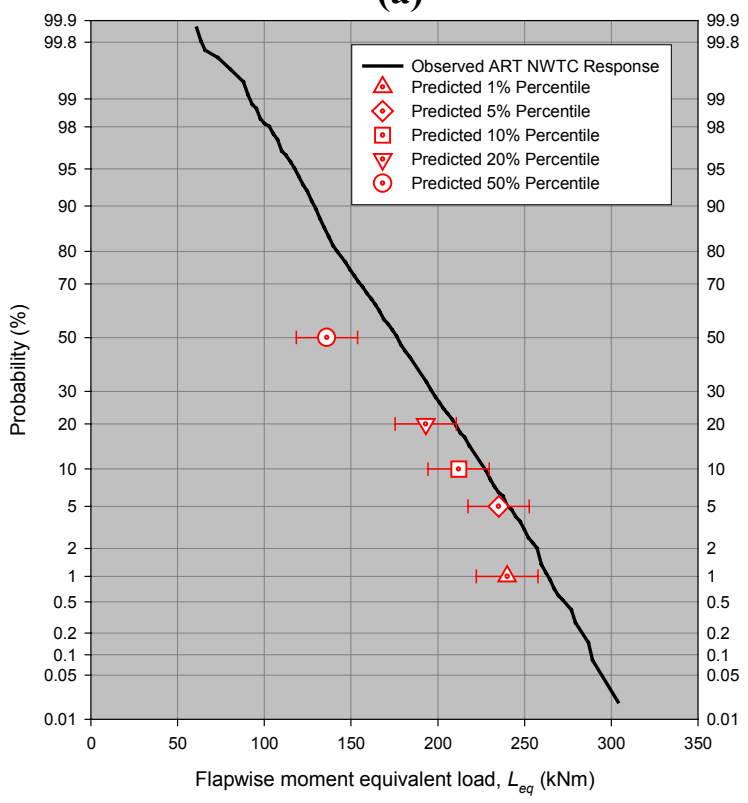

(c)

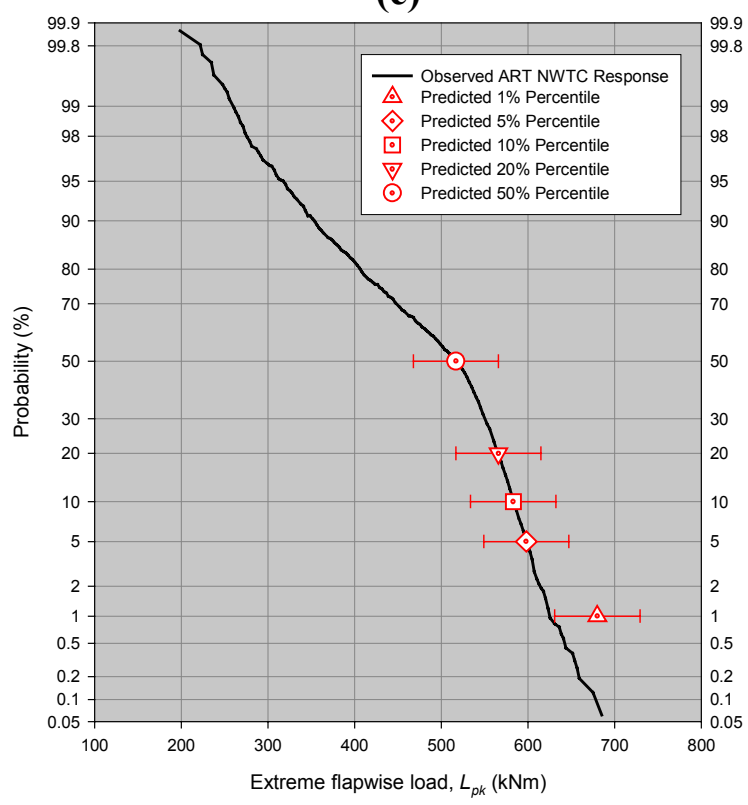

(b)

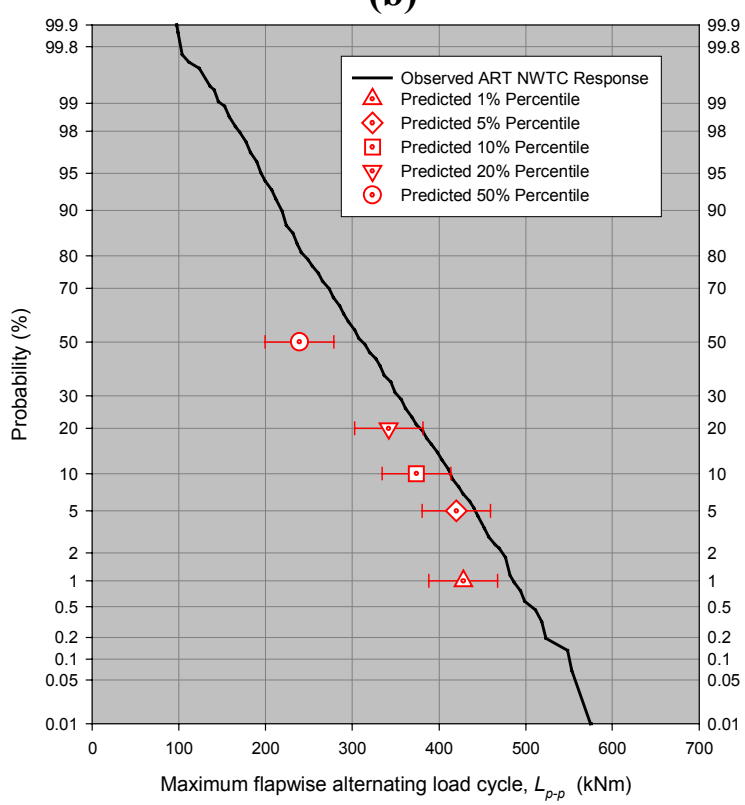

(d)

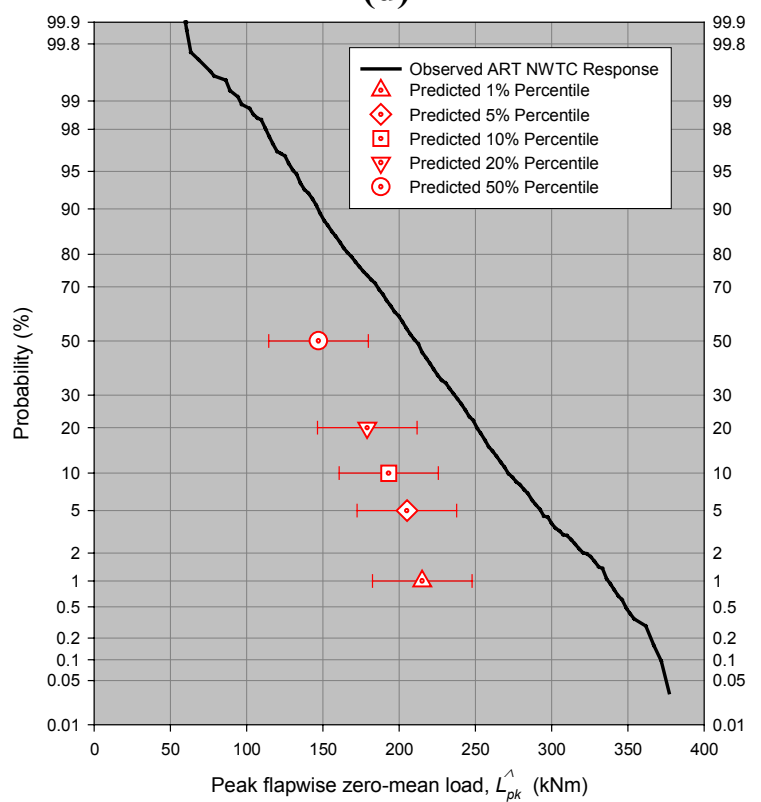

Figure 7-8. Comparison of NWTC/LIST ART turbine root flapwise (a) equivalent load $L_{\text {eq }}$, (b) maximum alternating load cycle $L_{p p}$, (c) extreme load $L_{p k}$, and (d) peak zero mean load $\hat{L}_{p k}$ cumulative probability distributions for stable flows with those predicted using LLLJP measured scaling parameters 


\section{Interaction of WINDPACT Turbine with a KH Billow}

In this section, we briefly discuss our initial efforts using NWTC/LIST and LLLJP measurements to improve the ability of the SNLWIND-3D turbulence code to more accurately represent inflow conditions that contain organized or coherent turbulent structures. Such structures, as discussed in Section 1.2.4, are responsible for inducing large loading events in simulated turbine responses and are currently not being adequately modeled. Not including such events at all or under representing them in inflow simulations has been demonstrated to cause fatigue damage estimates to significantly fall short of actual values. In previous sections, we demonstrated that the presence of a low-level jet (LLJ) maximum 200-300 m above the ground can be responsible for creating intense vertical shears below the height of the jet maximum. These flows can either remain stable with low turbulence levels, breakdown into energetic coherent turbulent motions, or generate long-period shear-gravity waves that periodically produce coherent turbulent bursts or eventually decay into organized turbulent structures.

We discussed (Section 2.5) that atmospheric wave motions are very common in the stably stratified boundary layer (usually seen at night) and that under the right circumstances of vertical wind speed (shear) and temperature distributions, a type of a flow process called KH instability may develop. If suitably triggered, such an instability can develop into largely 2-D motions called KH billows (waves), which eventually breakdown into the intense coherent structures that we can induce significant loads on wind turbine structures and components. We believe that we need to assess the impact of $\mathrm{KH}$ billow (waves) being ingested by operating wind turbines because: (1) of the ubiquitous nature of these flow structures in nocturnal stably stratified flows found beneath LLJs, and (2) there is evidence of such activity (Section 6) wherein such wave motions (probably KH waves) are the likely source of the coherent turbulence measured at both the NWTC and at the LLLJP Site. The high levels of root flapwise loads seen in stable flows where the background $R i<0.1$ and local vertical shears are significant (turbine layer or rotor layer power law exponents $>0.2$ ) point in that direction.

We will now briefly discuss a sample of our initial results of interfacing the NCAR KH billow simulation as the inflow to a turbine dynamic simulation. To do this, we employed a dynamic simulation of a 1.5-MW virtual turbine used as part of the WindPACT (Wind Partnership for Advanced Component Technologies Project). We have currently run a series of cases in which the mean wind speed and the shear across the rotor were varied. The intensity of the shear determines the time period of the KH billow or wave (i.e., the higher the shear, the shorter the lifetime). Higher shears produced a shorter, but more intense wave structure. We are in the process of comparing the model predictions of loads using inflows based on constant-speed, constant-shear, the IEC Normal Turbulence and Wind Profile Models (NTM/NWP), the SNLWIND-3D code scaled by the same boundary conditions as the NCAR KH simulation, and the NCAR KH simulation alone.

\subsection{The WindPACT 1.5-MW Baseline Turbine Test Bed}

We used the FAST_AD/AeroDyn $[34,35]$ model of a virtual three-bladed upwind 1.5-MW baseline turbine devised by D.J. Malcolm of Global Energy Concepts (GEC) and A.C. Hansen of Windward Engineering as part of the "WindPACT Turbine Rotor Design Study" [36]. This virtual machine was intended to exhibit many of the design attributes of 1.5-MW upwind, pitchregulated, three-bladed turbines currently in, or soon to be in, production. This variable-speed 
baseline design was used as the reference to assess the ability of a variety of possible improvements in the rotor system and its manufacture that had the potential to reduce the cost of energy (COE). Specifically the baseline design included the following design and operational features (see [36] for more details):

- Three blades

- Upwind

- Variable speed via full-span, collective-pitch control

- Rigid hub

- $\quad$ Hub height $=84 \mathrm{~m}$

- $\quad$ Rotor diameter $=70 \mathrm{~m}$

- $\quad$ Maximum tip speed $=75 \mathrm{~m} / \mathrm{s}$

- $\quad$ Design wind regime $=$ IEC class 2

- Maximum rotor speed $=20.5$ RPM.

The turbine simulation, as run, did not have a yaw degree of freedom (locked yaw). J. Jonkman [37] provided us with calculated non-rotating mode shapes. This particular turbine configuration was used because it provided many of the design and operational features seen in current 1.5MW upwind turbines. It is similar in many respects to the GE Wind $1.5 \mathrm{~S}$ turbine that will be deployed at the LLLJP Site.

\subsection{Interfacing the NCAR KH Billow Simulation to the AeroDyn Code}

The non-dimensionalized results of the KH billow simulation generated by the NCAR LargeEddy Simulation (LES) Code produced 233 GB of output. It was necessary to devise a methodology to obtain a subset of the original solution that was dimensionalized by the desired boundary conditions (background mean wind speed and vertical shear) that could be inserted into the AeroDyn [31] aerodynamics subroutine used with the FAST_AD Code. The interface developed not only dimensions the original solution, but also allows the user the choice of matching a selected portion of the most active part of the computational domain to all or part of the turbine rotor disk as well. In our first simulations, we matched the dimensions of active domain to the simulated rotor dimensions (i.e., the billow or wave dimensions are the same as the rotor disk). We did this because our results in Section 6 indicated the greatest response in the ART turbine occurred with a buoyancy length scale or characteristic wave size $\left(l_{b}\right)$ equal to the diameter of the rotor. The NCAR simulation was calculated using a background $R i$ value of +0.05 , which is within the weakly stable range discussed previously.

\subsection{Example of Using NCAR KH Billow As Input to Turbine Dynamic Simulation}

We now briefly discuss a few of the results obtained using the NCAR KH billow simulation as the inflow to FAST_AD simulation of the WindPACT 1.5-MW Baseline Turbine. The KH billow simulation was scaled to encompass the entire turbine rotor. The background mean hubheight wind speed was $17 \mathrm{~m} / \mathrm{s}$. This speed is above the rated value of $12 \mathrm{~m} / \mathrm{s}$, so the peak power was actively modulated by the control system. The background mean wind speeds at the bottom and top of the rotor were 12 and $16 \mathrm{~m} / \mathrm{s}$, respectively (a shear exponent of 0.578 ). The time 
period of the billow for the boundary conditions of mean wind speed and speed difference across the wave (rotor) was $301.7 \mathrm{~s}$. The first 30 seconds of the simulation were not used in the final solution because this time is necessary to allow the pitch control algorithm to properly settle from the initial startup transient. Thus, actual period of wave excitation of the turbine model was $271.7 \mathrm{~s}$. There are a total of 6000 time steps in the original NCAR solution. The actual usable output began at step 566, which was 28.5 seconds in scaled time into the original simulation.

\subsubsection{Simulated Inflow Description}

The non-dimensional temperature field of the entire $x-z$ domain is pictured in Figure 8-1 at 0 , $91.5,166.2,217.7$, and 271.7 seconds, where zero is assigned to the first record used by the turbine simulation after allowing for the pitch algorithm to settle. The $z$-axis height of each graph corresponds to the height of the wind turbine rotor diameter. The vertical black dashed line represents the location of the rotor $(x-y)$ plane, which is presented as the inflow to the turbine simulation $(y=90)$. As previously, the temperature is scaled relative to the color bar at the bottom with dark blue being the coldest and deep red the warmest.

At $t=0$, the billow circulation has already begun to form with colder and warmer air above and below each other within the circulation. As discussed previously, this creates a dynamic instability as a result of the buoyancy forces created. While some circulation is still visible at $t=$ $91.5 \mathrm{~s}$, turbulent mixing is becoming dominant as the temperature gradients are being smeared out. The billow circulation has completely broken and rolled over at $t=166.2 \mathrm{~s}$ and small, intense sub-circulations are visible. At $t=217.7 \mathrm{~s}$ a vertical temperature gradient is gradually reappearing, though there are intense circulations near the upper and lower boundaries. Finally, at $t=271.7 \mathrm{~s}$, a more noticeable vertical positive temperature gradient has appeared but the intense circulations near the boundaries continue.

The graphs of Figure 8-2 depict the rotor plane ( $x$-y) distributions of local stability $(R i)$, shear stress $u$ 'w', turbulent kinetic energy $E$, and the coherent turbulent kinetic energy $E_{c o h}$. We will refer to the shearing stress $\tau=\rho \sqrt{u^{\prime} w^{\prime}}$ in the text and figures as $u^{\prime} w^{\prime}$ for convenience. The circle represents the rotor disk. In the graph plotting the local $R i$, dark blue represents the highest dynamic stability $(R i>0)$, white is neutral $(R i=0)$, and bright yellow convectively unstable $(R i<0)$. The bulk of the rotor disk is stable with the exception of two narrow bands that are unstable (convective) in the upper and lower halves with a neutral layer at the center. Turbulence production is taking place as a result of positive buoyancy within these narrow unstable layers. There is a narrow band of high shear stress values near the bottom of the rotor (shown in yellow) and a weaker one near the top in which mechanical turbulence production is taking place (where the local shear stress is indicated as $u^{\prime} w^{\prime}$ and either deep red or blue colors represent regions of high turbulence production). Not plotting the shear stress as $\tau$ has the advantage of retaining the sign (colors) that can be thought of as a crude indicator of the intensity of vertical motions in the $x$-z plane. These narrow bands also show up as kinetic energy (K.E.) ( $E$ and $E_{c o h}$ ) concentrations (the highest values of which are also shown in yellow) in the same vicinity of the shear turbulence production.

An analysis of the predicted turbine dynamics showed an initial significant response to the $\mathrm{KH}$ billow occurred near $t=91.5 \mathrm{~s}$. The same information contained in Figure 8-2 is presented in Figure 8-3 for this time step. Here small intense areas of convective turbulence have formed within the more stable disk area. Shear turbulence production $\left(u^{\prime} w^{\prime}\right)$ has become much more 
dispersed and is taking place throughout the disk. Small regions of intense turbulent and coherent turbulent K.E. have also formed in portions of the upper and lower halves of the disk.

The most significant turbine response was noted at $t=166.2 \mathrm{~s}$, after the billow appears to have broken and completed its roll over. This time step is shown in the same format in Figure 8-4. Here regions of local convective and intense mechanical (shear) turbulence production have developed near the center of the disk. These productive regions (particularly the shear) are highly correlated with intense coherent structures $\left(E_{c o h}\right)$ associated with regions of concentrated K.E $(E)$ in the lower right quadrant of the rotor disk.

The re-emergence of a weak vertical temperature gradient shown at $t=217.7 \mathrm{~s}$ is shown in Figure 8-1 and is reflected in the local $R i$ distribution of Figure 8-5. While the flow through the disk is becoming generally stable, there continues to be "hot spots" of convective activity and weaker shear stress regions. There remain two small regions of relatively intense $E_{c o h}$ in the lower left and upper right quadrants, even though the overall turbulent K.E. levels have decreased. Finally, Figure 8-6 displays the more noticeable vertical temperature gradient seen at time step $t=271.7 \mathrm{~s}$. Again, while most of the disk is locally stable, there continue to be small convective regions in the lower parts of the disk. Shear-generation turbulence is being produced throughout the disk, albeit at levels much lower than the peaks shown in Figure 8-4.

The time histories of the hub-height values of $U_{H}$; the turbulent components $u$ ', $v^{\prime}$, and $w^{\prime}$; the instantaneous Reynolds stresses $u$ 'w', $u$ 'v', and $v^{\prime} w^{\prime}$; and the time-varying $E$ and $E_{c o h}$ are plotted in Figure 8-7. This is what the parameter time series would measure when seen by a single sonic anemometer mounted upstream of the center of the rotor disk. Clearly, the presence of organized structures as identified by the Reynolds stress components and the K.E. parameters are notable. What is missing, however, is the remainder of the spatial distribution of these parameters that the rotor blades pass through that cannot be derived from a single point measurement. Some information about coherent structures is available from a single, hub-height location, as is shown in Figure 8-8. The turbulent structure of an isolated gust in $U_{H}$ is plotted in Figure 8-8a. The shear stress $u$ ' $w$ ' and K.E. ( $w^{\prime} E$ and $w^{\prime} E_{c o h}$ ) associated with this gust are clearly identified. A more complex picture of a later segment is presented in Figure 8-8b. We have seen both types of time series derived from actual single-point sonic anemometer measurements. But usually we have been unable to fully appreciate the large-scale flow structures that are responsible and how they may be affecting the operation of a wind turbine.

\subsubsection{Response of Turbine to KH Billow}

The response of the simulated WindPACT 1.5-MW baseline turbine as indicated by the zeromean root flapwise bending load and described by the combination of the continuous and discrete wavelet transforms is presented in Figure 8-9. This is the same format in which we described the responses of the Micon 65/13 and CWE300 turbines to coherent turbulence in Section 1.2.4. Here the detail bands D4 through D9, shown at the bottom, correspond to frequency ranges of 6.25 to 12.5 (D4), 3.13 to 6.25 (D5), 1.56 to 3.13 (D6), 0.78 to 1.56 (D7), 0.39 to 0.78 (D8), and 0.20 to 0.39 (D9) Hz, respectively. The ordinate axes of these bands are scaled in units of $\mathrm{kNm}$.

Immediately noticeable in Figure 8-9 is the reduction in the low-frequency dynamic stress below $0.4 \mathrm{~Hz}$ at about $160 \mathrm{~s}$ and the corresponding decrease in the amplitude shown in detail band D9. This band contains the blade passage (1-P frequency) of $0.33 \mathrm{~Hz}$ (at $20.5 \mathrm{rpm}$ ), as well as the 
first tower side-to-side and fore-aft mode shapes. The decrease in the 1-P load is the result of the mixing out of the vertical shear by the turbulence generated by the breaking wave. This decrease in the mean vertical shear across the rotor layer is plotted in Figure 8-10 and clearly demonstrates the consequences of this mixing process, which in turn lowers the 1-P stress levels seen in Figure 8-9.

The bursts of stress energy in the roughly 0.7 to $1.5 \mathrm{~Hz}$ frequency range (shown also in the D7 and D8 detail bands) up to $120 \mathrm{~s}$ are responses of the first blade axisymmetric and first blade symmetric flap mode shapes. The wave breakdown begins at about $135 \mathrm{~s}$ and continues until about $180 \mathrm{~s}$, as shown in Figure 8-7. During this time period, the higher order modes $\left(1^{\text {st }}\right.$ and $2^{\text {nd }}$ blade axisymmetric edge, flap/tower coupling, $2^{\text {nd }}$ blade axisymmetric, and $2^{\text {nd }}$ blade symmetric and axisymmetric edge) in the 1.5 to $6 \mathrm{~Hz}$ band also exhibit bursts of stress energy, as seen in the D5 and D6 detail bands. Some of this stress energy is occurring concurrently (superposition). It is during this period that the flow entering the rotor was described by the graphs in Figure 8-4. The inflow at this time is likely filled with energetic turbulent structures that have dimensions much less than the rotor diameter. These structures are responsible for exciting the higher order modes. As the energetic turbulence dies away after about $200 \mathrm{~s}$ and as the stable temperature gradient begins to re-establish itself, the excitation of the mode shapes also decreases in both in occurrence and intensity, as seen in Figure 8-5. By the end of simulation at $t=271.7 \mathrm{~s}$, all modal excitation above about $0.7 \mathrm{~Hz}$ has ceased, and only the tower fore-aft and side-to-side modes are responding to the decreased, but still present, wind shear (shear exponent $\sim 0.4$ ). While the inflow structures shown in Figure 8-6 for this time step appear very energetic from the color codes used, a comparison with Figure 8-4 will reveal the actual magnitudes are much smaller, particularly in the shear stress ( $u$ ' $\left.w^{\prime}\right)$ and $E_{c o h}$ fields.

We now discuss our initial progress in comparing the model predictions of loads using inflows based on:

- Constant-speed, constant-shear (shear is the same as the NCAR KH simulation)

- The IEC Normal Turbulence and Shear Models (NTM/NWP)

- The SNLWIND-3D code scaled by the same boundary conditions as the NCAR KH simulation and referred to as "background turbulence"

- The NCAR KH billow lifecycle simulation alone.

The root flapwise bending moment frequency spectra for each of these simulations are plotted in Figure 8-11. We compare the response root flapwise load spectra for the constant-speed, constant shear, and the background turbulence simulated by the SNLWIND-3D Code in Figure 8-11a. The constant-speed/shear inflow concentrates the bulk of the stress energy at once per revolution (1-P) with much, much smaller amounts at blade passage harmonics. The background turbulence also concentrates the highest energy at 1-P as a result of the same level of shear as the constant-speed/shear case. Above 1-P, the turbulence exhibits a random decay with no discrete frequencies present.

In Figure 8-11b, we compare the response spectra related to the background turbulent and IEC NTM/NWP inflow simulations. Here the discrete peak at 1-P in the background flow is apparent, but there is no equivalent one from the NTM flow because of the much lower (NWP) rotor disk shear exponent specification (0.2). The response generated by both inflows is random with a decaying spectral density with increasing frequency; the average level is higher for the 
NTM/NWP simulation. This a consequence of the NTM/NWP specification of neutral stability $(R i=0)$ as a boundary condition, whereas the background turbulence is scaled as weakly stable by $R i=+0.05$.

We now compare the response spectra for the KH billow lifecycle excitation with the background turbulence in Figure 8-11c. It should be pointed out that an actual KH billow never exists by itself in a real atmospheric flow. It is always embedded within a much more random background turbulence field. We simulate that background turbulence with the SNLWIND-3D code using the same scaling as for the KH wave (i.e., mean wind speed, vertical shear, and stability). Thus, in the real world, the flow structure and turbulence associated with the KH wave is superimposed upon the background turbulence. Eventually the breakdown and mixing process absorbs the additional turbulent energy as part of the overall flow, and the coherent structure gradually decays into a random field. Thus, the inflow structure that the turbine rotor sees over the lifecycle period of the wave will be the sum of the coherent turbulence associated with the KH billow lifecycle. The background and the response spectra shown in Figure 8-11c will be the sum of the two shown (assuming linear response processes are dominant). Clearly, the root flapwise response caused by the KH billow alone contains a number of harmonically related discrete spectral peaks that do not exist in the response related by the much more random background turbulence. These discrete peaks are the result of the rotor encountering the temporally and spatially coherent structure elements of the KH billow and the distribution of coherent energy into circulations smaller than rotor disk dimension, as exemplified in Figure 8-4. The harmonic relationship seen is likely the result of the excitation being applied as a coherent narrowband excitation to the range of mode shapes. This can be seen in the wavelet analysis detail bands D4 through D7 of Figure 8-9 in the period between 140 and $180 \mathrm{~s}$.

In Figure 8-11d, we present the comparison between the response spectra from the turbine being excited by the KH billow lifecycle and the IEC NTM/NWP. Again, we see the results of a narrowband excitation being superimposed on top of a more wideband process. The only difference between this case and the previous one is that the background turbulence level is higher and it does not contain the $1 / \mathrm{rev}$ peak. Because of the logarithmic ordinate, there is considerable energy associated with the areas under the discrete frequency peaks and it is being applied coherently (in phase). The turbulence energy being applied is occurring in short, intense, coherent bursts in one case and with a random phase in the other. Whether or not that makes any difference from a structures or fatigue viewpoint probably depends on response characteristics of the structural element involved.

We will continue to expand our effort in examining the role of the wave-induced turbulence on turbine response in the nocturnal boundary layer. Our next step is to analyze in more detail how fatigue damage is influenced by the application of a $\mathrm{KH}$ billow lifecycle to the simulated turbine inflow. 


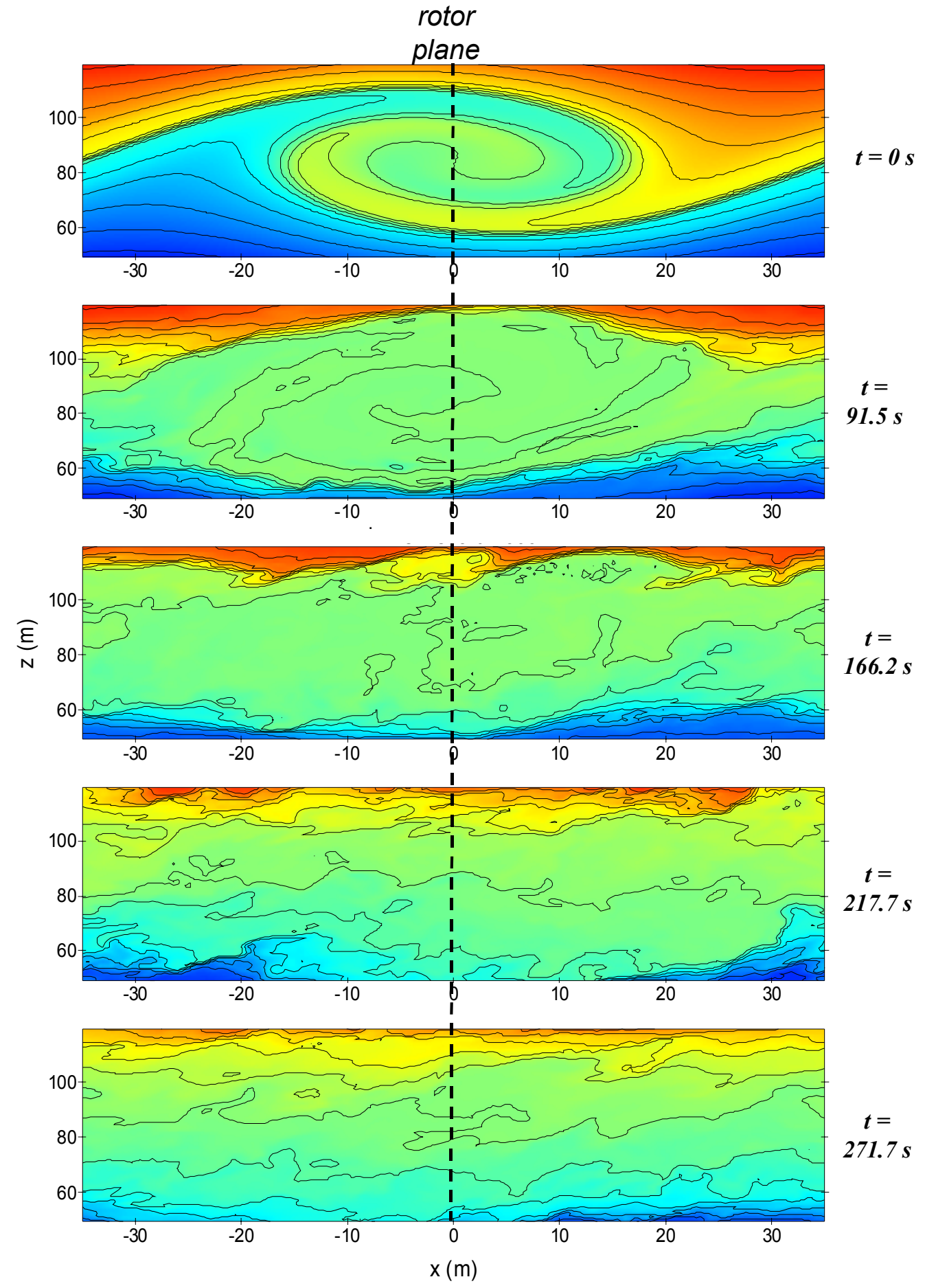

Figure 8-1. Evolution of the KH billow used as input to the simulation of the WindPACT 1.5-MW baseline turbine 


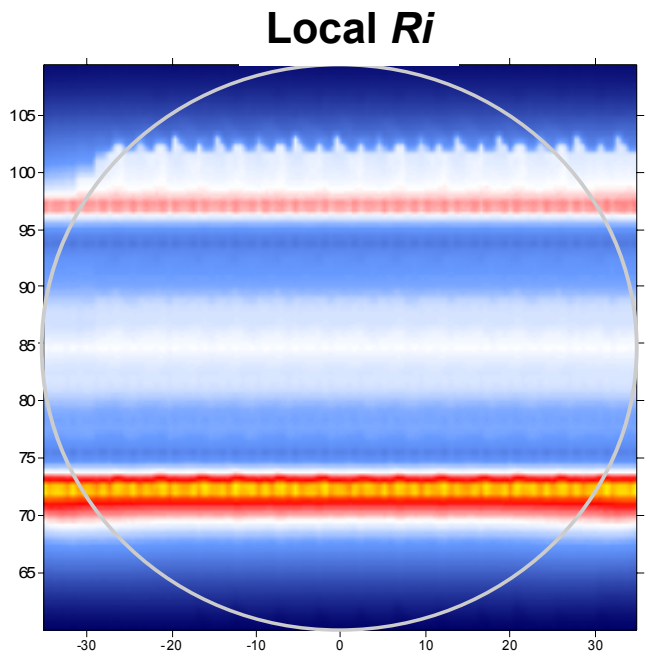

$$
t=0 s
$$
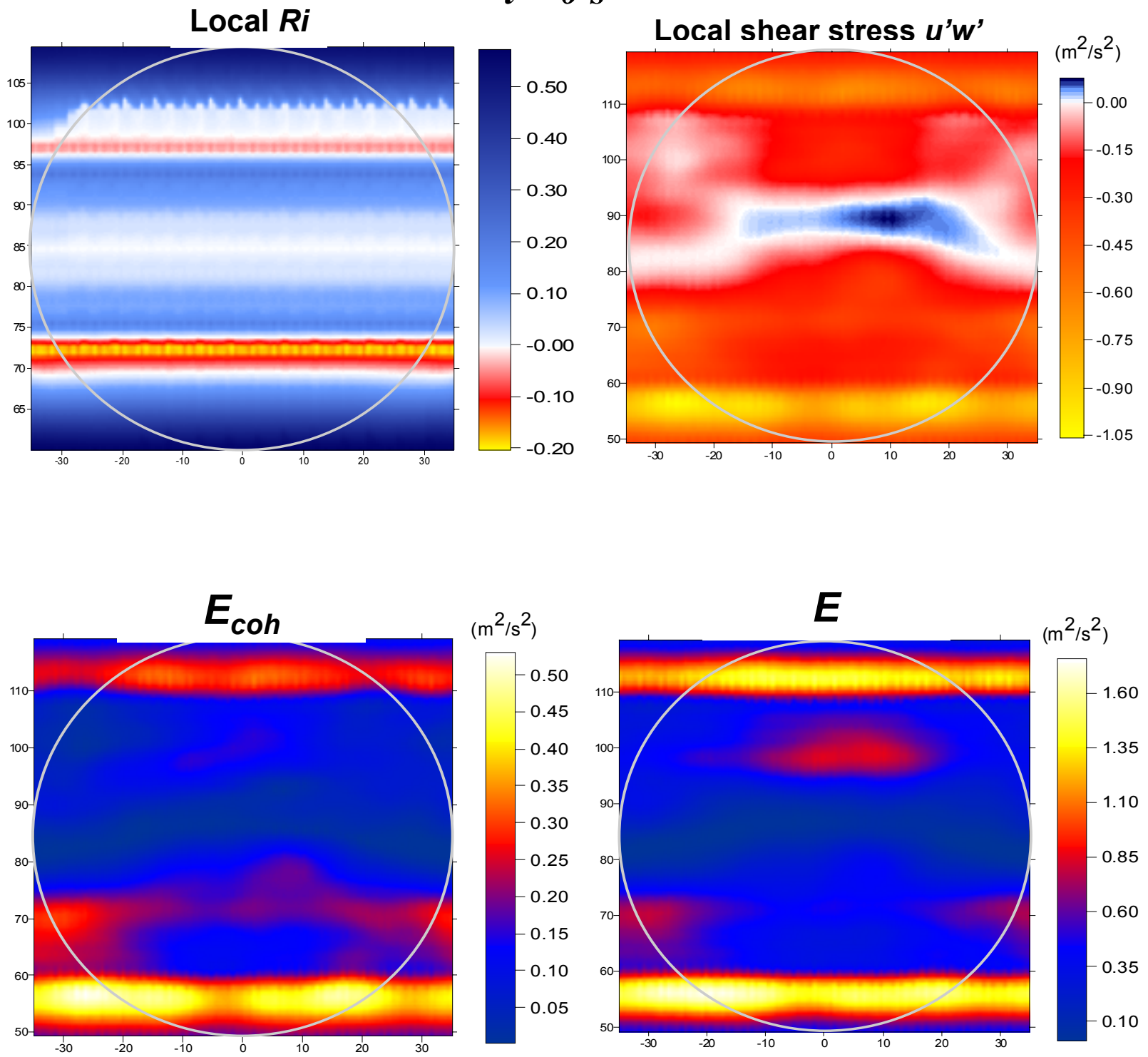

Figure 8-2. Rotor plane distributions of local $R i$, shear stress $u^{\prime} w^{\prime}, E_{c o h}$, and $E$ at $\mathrm{t}=0 \mathrm{~s}$ 


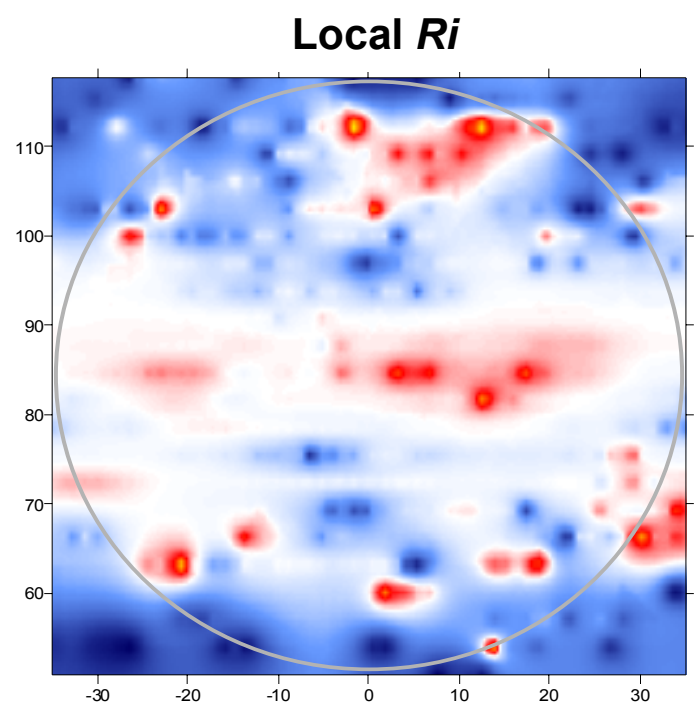

$$
t=91.5 \mathrm{~s}
$$

\section{Local shear stress $u^{\prime} w^{\prime}$}
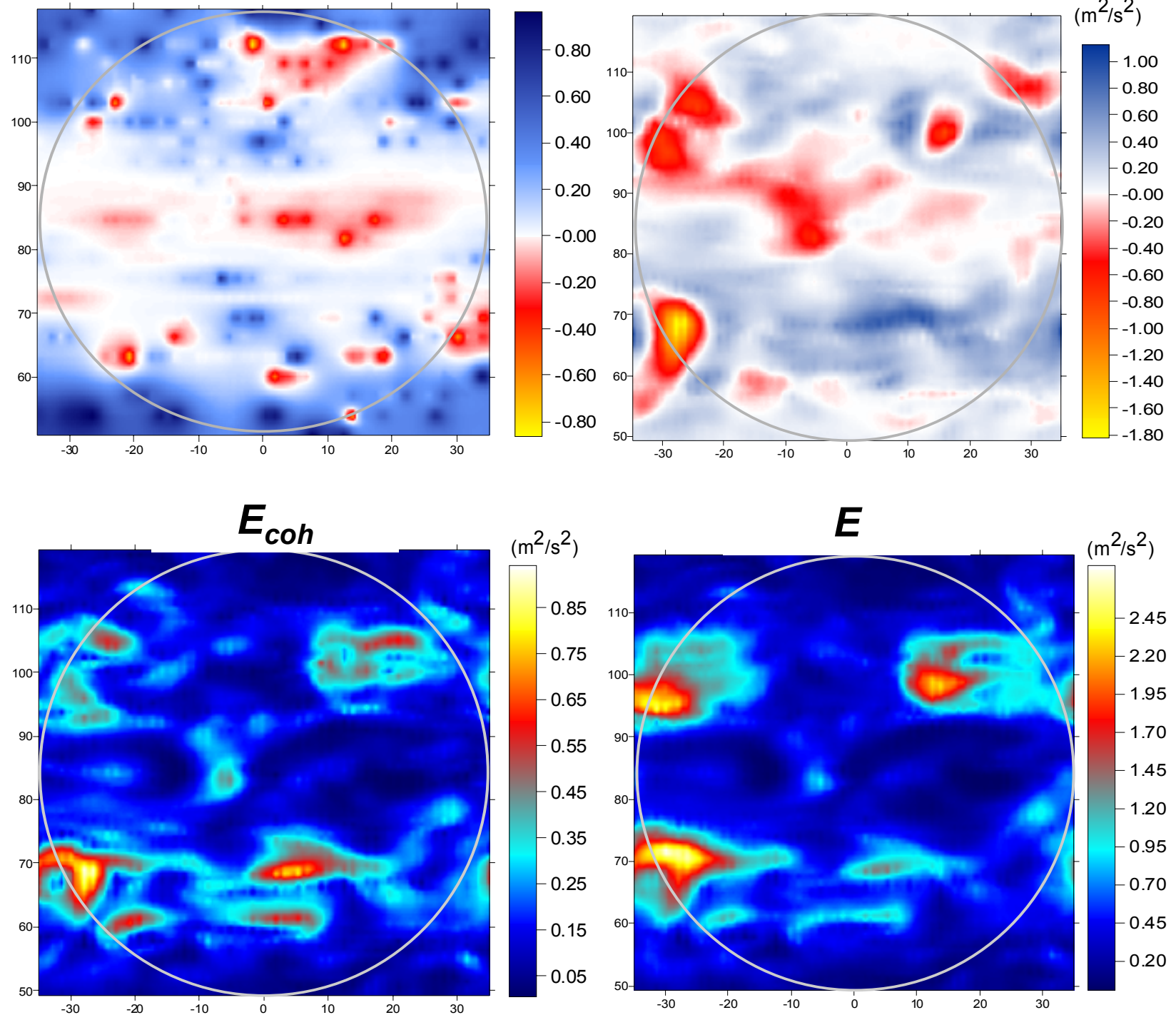

Figure 8-3. Rotor plane distributions of local $R i$, shear stress $u^{\prime} w^{\prime}, E_{c o h}$, and $E$ at $\mathrm{t}=91.5 \mathrm{~s}$ 

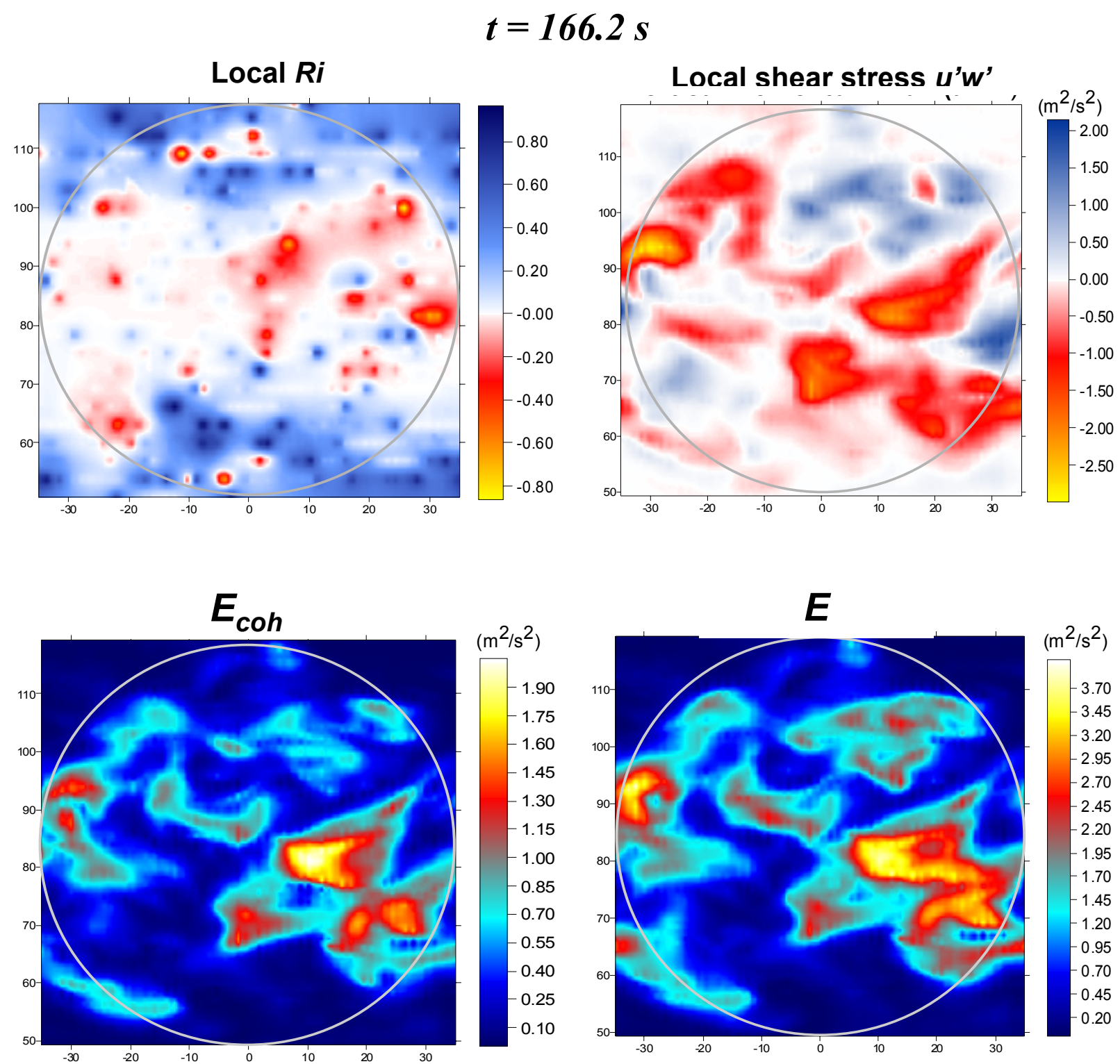

Figure 8-4. Rotor plane distributions of local $R i$, shear stress $u^{\prime} w^{\prime}, E_{c o h}$, and $E$ at $t=166.2 s$ 

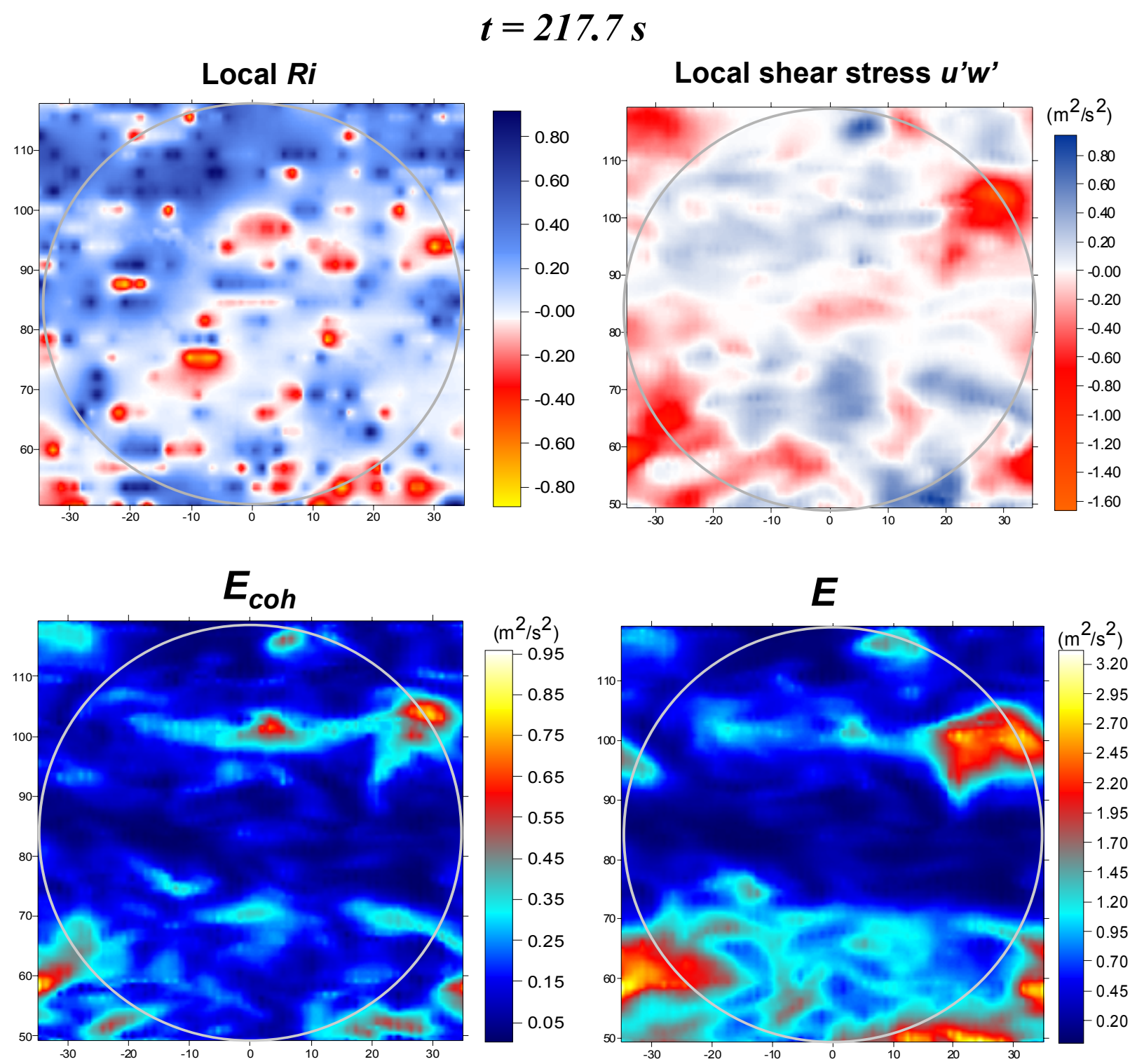

Figure 8-5. Rotor plane distributions of local Ri, shear stress $u^{\prime} w^{\prime}, E_{\text {coh, }}$, and $E$ at $\mathrm{t}=217.7 \mathrm{~s}$ 


$$
t=271.7 s
$$
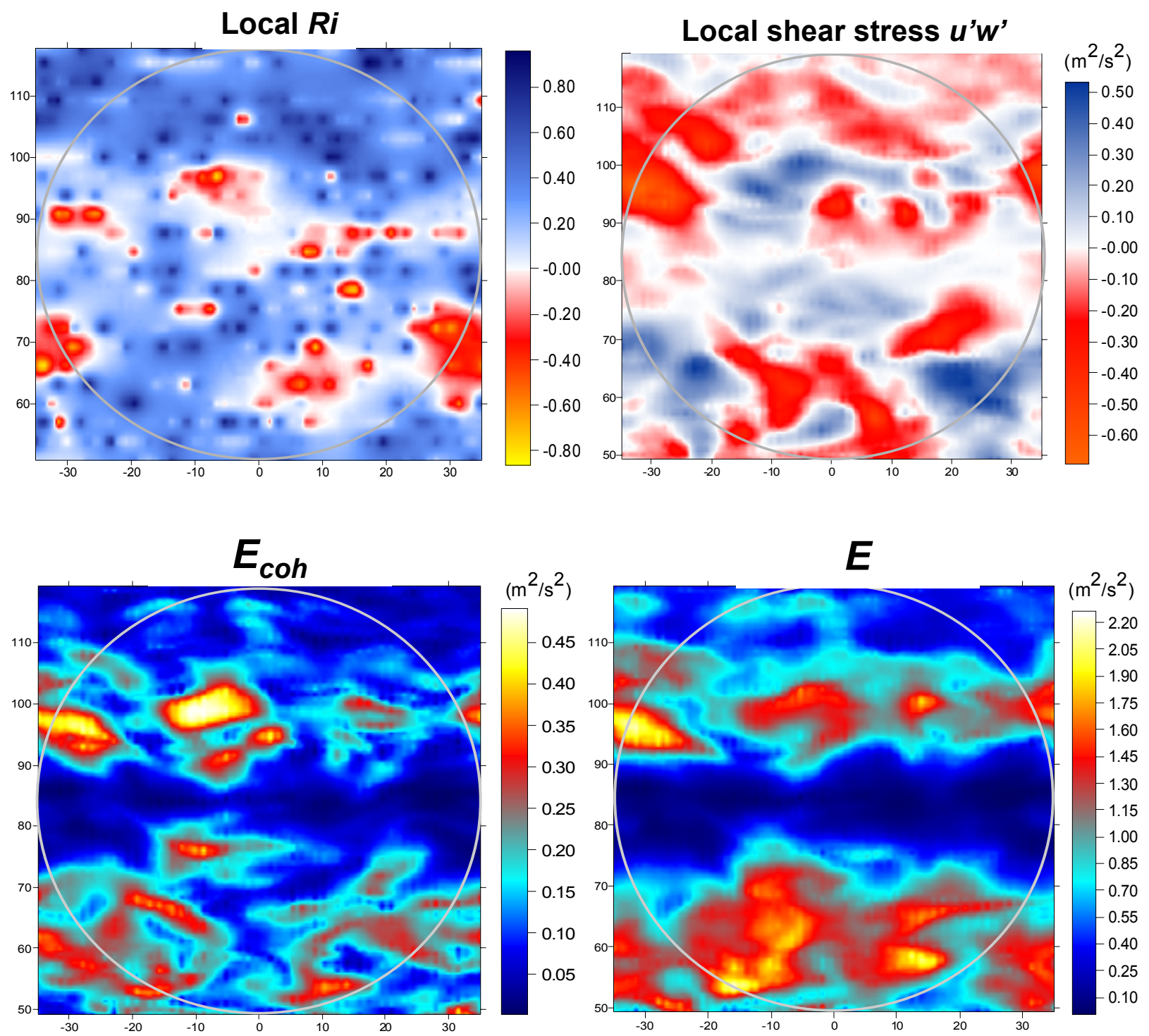

Figure 8-6. Rotor plane distributions of local $R i$, shear stress $u^{\prime} w^{\prime}, E_{c o h}$, and $E$ at $t=271.7 \mathrm{~s}$ 

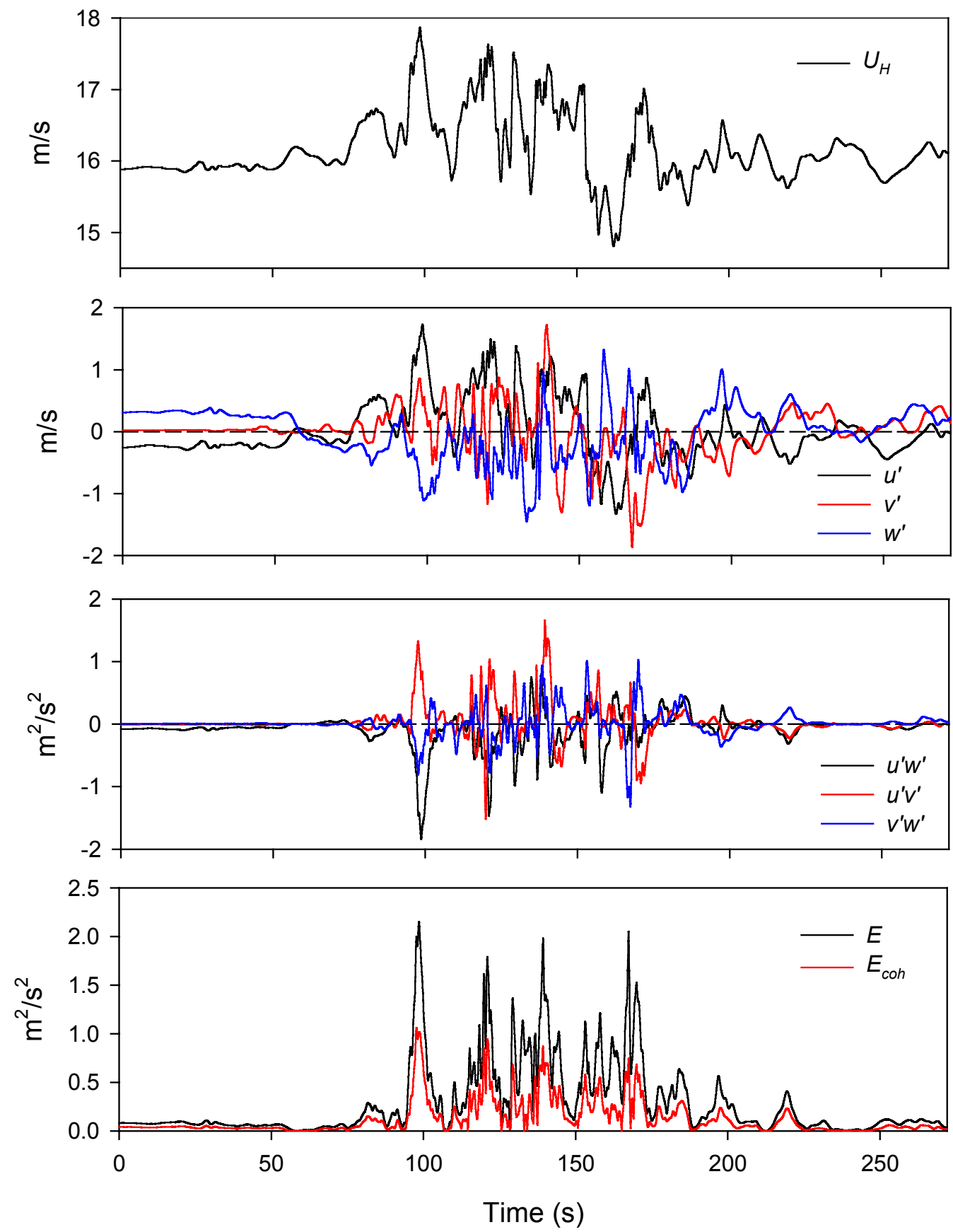

Figure 8-7. Time histories of hub-height $U_{H}, u^{\prime}, v^{\prime}$; and $w^{\prime}, u^{\prime} w^{\prime}, u^{\prime} v^{\prime}$; and $V$ 'w' and $E$ and $E_{\text {coh }}$ 

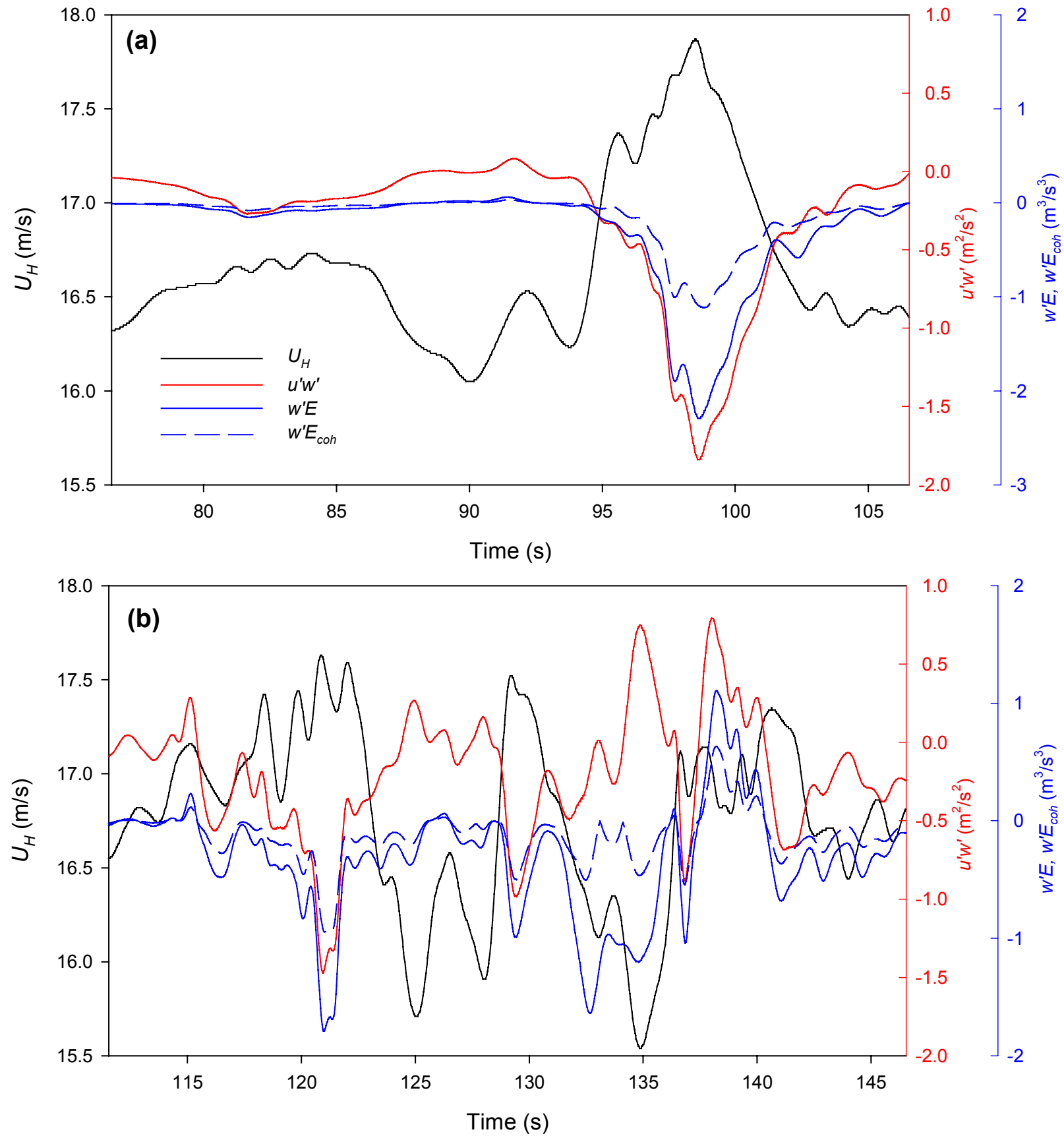

Figure 8-8. Time histories of hub-height $U_{H}$, shear stresses $u^{\prime} w^{\prime}, w^{\prime} E$, and $w^{\prime} E_{c o h}$ for two periods 


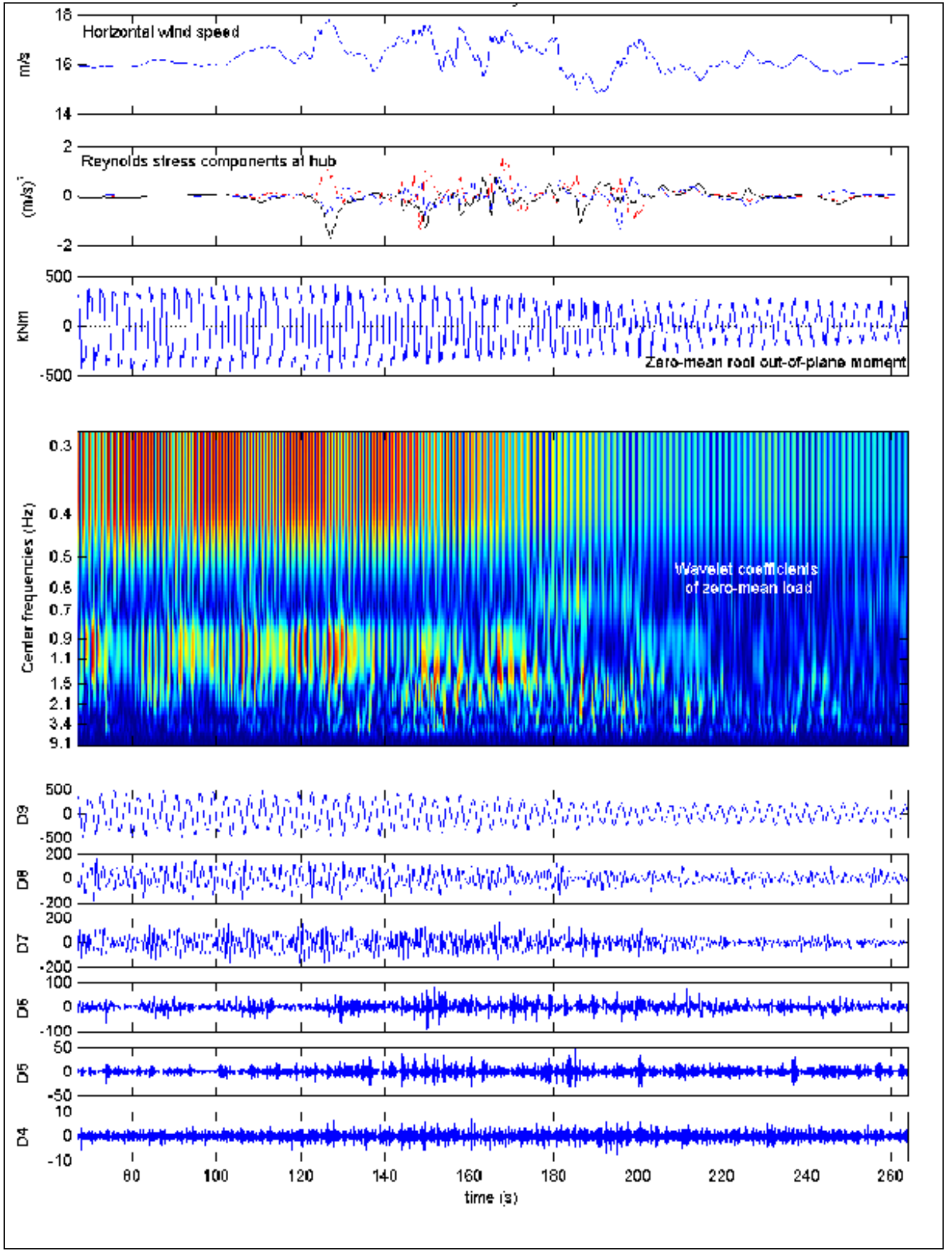

Figure 8-9. Time and wavelet analysis of simulated WindPACT 1.5-MW baseline turbine KH billow turbulence/rotor interaction 


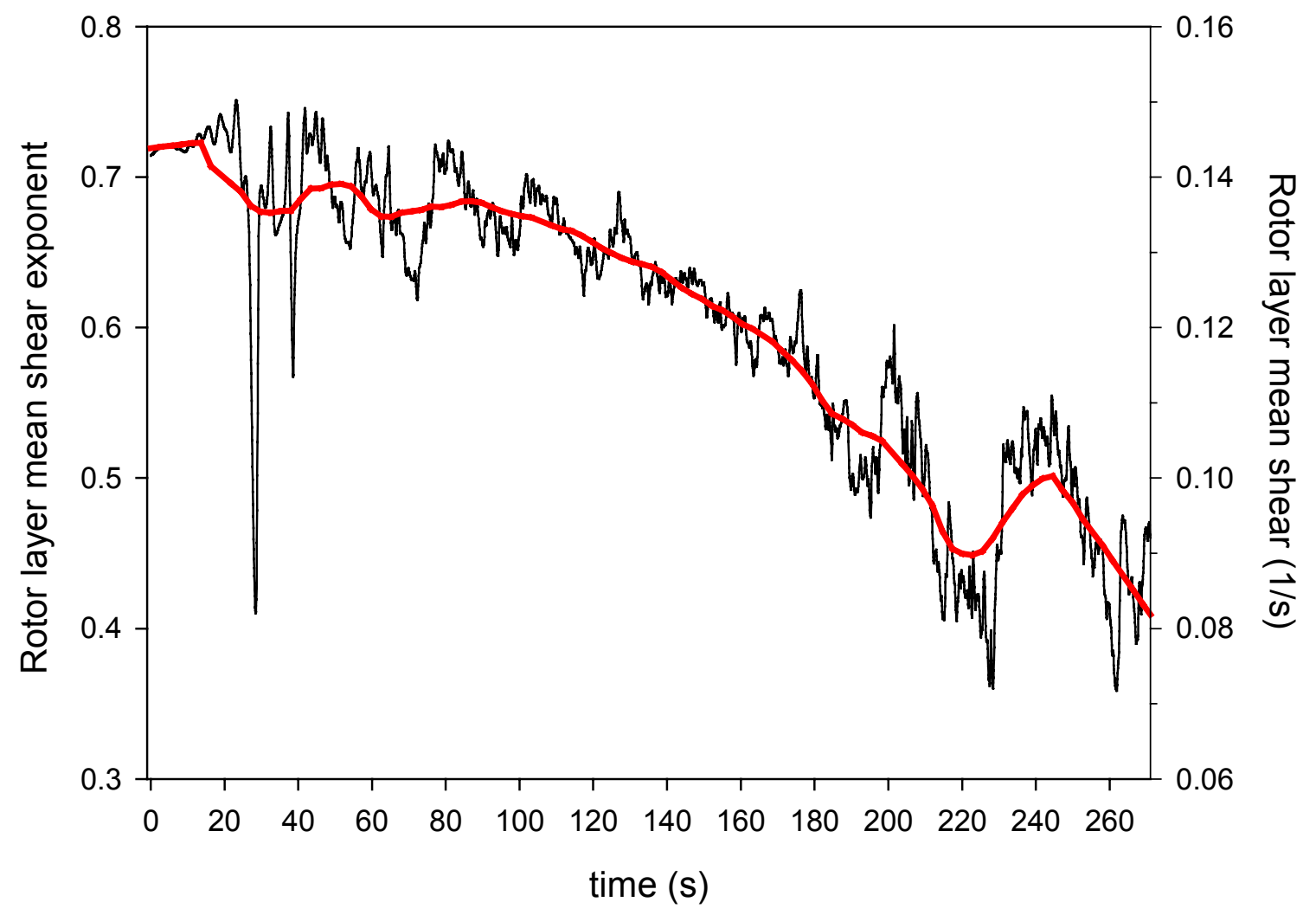

Figure 8-10. Decrease in rotor layer mean vertical shear during lifecycle of $\mathrm{KH}$ billow 

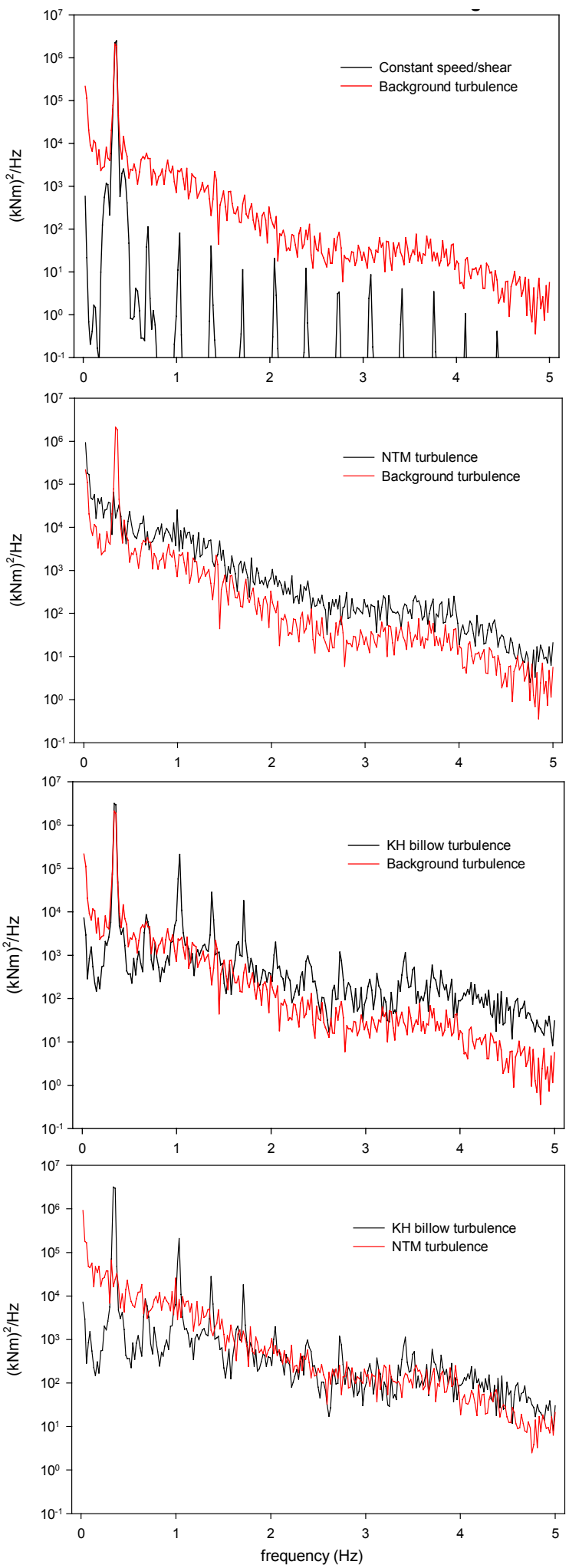

Figure 8-11. Comparison of predicted root flapwise bending load spectra for constant speed/shear, SNLWIND-3D background turbulence, IEC NTM/NWP turbulence, and KH billow lifecycle inflows 


\section{Initial Conclusions}

We have analyzed a full year of records of statistical data collected over 5 -minute intervals from NREL instrumentation installed on the GE Wind 120-m meteorological tower, located on the western edge of the Great Plains south of Lamar, Colorado, as part of the Lamar Low-Level Jet Program (LLLJP). We have also analyzed in detail three representative case studies of commonly occurring conditions in which a low-level jet stream was present above the tower when wind profile information was available from an acoustic wind profiler or SODAR. We employed an analysis of data collected from the NWTC/LIST Program using the ART turbine in order to develop guidance criteria to assess possible turbine operational impacts based on the measurements obtained from the GE Wind tower. We constructed a simple linear regression model of the ART blade root flapwise load response in order to identify a set of inflow parameters to help explain a high percentage of the variation of that response. Using this model as a tool and based on our analysis of both the long-term record and case studies from the GE Wind tower, we have reached the following initial conclusions from the following program elements.

\subsection{NWTC/LIST Program}

- Large flapwise load responses at the blade root are associated with a narrow range of dynamic stability of the atmospheric layer occupied by the wind turbine.

- Length scales of atmospheric wave motions existing within this stability range close to the dimensions of the turbine rotor ensure a dynamic coupling of turbine higherorder resonant modes with inflow coherent turbulent structures of similar spatial dimensions.

- The hub-height mean wind speed, standard deviation of the vertical wind speed, and the peak value of coherent turbulent kinetic energy, when coupled with the dynamic stability of the layer from the ground to the top of the turbine rotor, were found to be an efficient set of predictors of the statistical response of the blade root flapwise loads.

\subsection{One-Year LLLJP Statistical Data Base Analysis}

- The highest available energy density at the LLLJP Site occurs in late spring and early summer (April - June).

- The highest turbulence intensities occur in the warm season (April through September) with the highest mean values (10-12\%) occurring in the 52- to $113-\mathrm{m}$ (rotor) layer during June and July.

- The observed mean turbulence levels (wind speed standard deviation) at the tower 52 $\mathrm{m}$ and $113 \mathrm{~m}$ measurement heights remain below the IEC " $\mathrm{A}$ " and " $\mathrm{B}$ " specifications.

- The highest turbulence intensities are seen when the 52- to 113-m (rotor) layer gradient Richardson number is in the range $0<R i<0.25$.

- The 0.1 percentile of the peak gust or extreme velocity with the turbine rotor layer $(52-113 \mathrm{~m})$ is $30 \mathrm{~m} / \mathrm{s}$. 
- The distribution of vertical mean shear over the rotor layer is positively skewed with a significant tail that frequently exceeds the IEC Normal Wind Profile (NWP) specification.

- The annual shear exponent probability density distributions can be approximated by two Gaussian distributions: one for shear exponents $\leq 1 / 7$ and the other for exponents $>1 / 7$.

- Shear exponents $>1 / 7$ in the rotor layer occur frequently with vertical stabilities in the critical range (i.e., $0<R i<0.25$ ).

- Extreme values of mean shear decrease as the mean wind speed increases.

- The highest vertical shears seen by operations turbines at this site will occur most often with a hub mean wind speed in the range of 8-14 m/s.

- The warm season (April-September) is dominated by southerly winds in which low-level jets (LLJs) are known to form frequently at night.

- Potentially challenging flow conditions within the rotor layer can be expected to occur up to $16 \%$ of the time annually when the layer is critically stable, the shear exponent is in excess of 0.2 , and the hub mean wind speed is between 8 and 13 $\mathrm{m} / \mathrm{s}$.

- Weakly stable inflow conditions that are associated with significant turbine structural response existed a total of 600 hours with two-thirds of that total occurring during the warm season.

\subsection{Case Study Results}

- The presence of LLJs can significantly modify the vertical shear and turbulence environments within the vertical layer occupied by turbine rotor disks (rotor layer).

- The presence of a LLJ does not always create significant levels of coherent turbulence in the turbine rotor layer.

- LLJs can create intense vertical shears to altitudes of at least $200 \mathrm{~m}$.

- Depending on atmospheric conditions, the presence of a LLJ can cause intense shears to become unstable and breakdown into intense coherent turbulence.

- The height of a LLJ maximum can vary during the night, which can modulate the intensity of the vertical shear and turbulence conditions encountered in the layer where the turbine rotors reside.

- The stably stratified shears that exist below low-level jets can support the development of various forms of shear flow instabilities including KelvinHelmholtz (KH).

- We believe that KH instability and the accompanying billow formations are a common occurrence in the nocturnal boundary layer at the LLLJP Site and especially when low-level jets are present. 
- Applying our simple ART regression response model to LLLJP measurements suggests that conditions at this site will induce similar statistical levels of response seen at the NWTC in the ART turbine as well as other turbine designs.

- Breaking atmospheric wave motions in the nocturnal boundary layer are likely a major source of coherent turbulence at this site.

- It will be necessary to include KH billows in the SNLWIND-3D code in order to provide a representative inflow turbulence simulation for the LLLJP Site.

- At this point it is unlikely that an acoustic wind profiler (SODAR) by itself will be of much use as an operational early warning device of coherent turbulence activity because of its intermittent ability to provide the necessary wind profile information.

- It does appear likely that some form of direct measurements may be required to provide such an early warning function (real-time vertical stability $R i$ and $E_{c o h}$ for example).

- The power law shear profile currently specified by the IEC NWP is inadequate for large rotors in the highly sheared environments associated with the presence of LLJs in which the shear profile varies with height.

- At a minimum, it will necessary to specify the vertical dynamic stability $(R i)$ as a function of height in the SNLWIND-3D code in order to reflect actual conditions of background turbulence seen at the LLLJP Site (local scaling).

\subsection{Interaction of a Turbine Rotor Blade with a KH Billow}

- The presence of a KH billow undergoing its lifecycle in the turbine inflow induces discrete peak loads in the response load spectrum.

- The discrete spectral peaks arise from the rotor blade encountering the temporally and spatially coherent turbulent structures of the KH billow circulations.

- The KH billow serves as a narrowband turbulence excitation source as compared with the more random wideband excitation induced by normal background turbulence generated by the SNLWIND-3D code and that specified by the IEC Normal Turbulence and Wind Profile Models (NTM/NWP).

- The breaking of shear-generated atmospheric wave motions are likely the ultimate source of the extreme loads that have previously been unaccounted for in simulations. 


\section{Future Work}

In the next 18-24 months, we expect to accomplish (providing sufficient resources are available) the following:

- Collaborate with the National Oceanic and Atmospheric Administration's Environmental Technology Laboratory using their high-resolution Doppler LIDAR to

- Assess the ability of a Doppler LIDAR for use in long-term wind resource assessment up to heights of $200 \mathrm{~m}$ above ground level

- Determine the usefulness of Doppler LIDAR for characterizing turbulence events and particularly those events that are likely to present some damage risk to wind turbine operations

O Assess the usefulness of using a forward-looking Doppler LIDAR to detect coherent turbulent structures in turbine inflows

- Investigate the horizontal distribution and dimensions of turbulence or wave packets in the nocturnal stable boundary layer

- Compare the usefulness of direct measurements in comparison with remote sensing via LIDAR and SODAR for support of wind farm operations

- Complete the data reduction of the more than 30,000 10-minute records of detailed turbulence information collected from the GE Wind 120-m tower from March 2002 through March 2003

- Use the detailed tower measurements, the SODAR observations (when available), and the NOAA LIDAR results to aid in improving the SNLWIND-3D turbulence code to better simulate conditions associated with shear flow instabilities (such as KH billows) frequently encountered in the nocturnal stable boundary layer

- Investigate obtaining vertical temperature profiles using radio-acoustic remote sensing (RASS) in conjunction with SODAR wind measurement to obtain real-time measurements of vertical stability and shear profiles near wind farms that may be used for operational purposes (i.e., control of loads from encounters with coherent turbulence). 


\section{References}

1. Sutherland, H.J. (January 2002). "Inflow and the Fatigue of the LIST Wind Turbine." Proceedings of 2002 ASME Wind Energy Symposium. Reno, Nevada. January 14-17, 2002. Fairfield, NJ: American Society of Mechanical Engineers.

2. Kelley, N.D. (November 1993). "The Identification of Inflow Fluid Dynamics Parameters That Can Be Used to Scale Fatigue Loading Spectra of Wind Turbine Structural Components." Prepared for $13^{\text {th }}$ ETCE Wind Energy Symposium, New Orleans, Louisiana, January 23-26, 1994. NREL/TP-442-6008. Golden, CO: National Renewable Energy Laboratory, 8 pp.

3. Kelley, N.D.; Wright, A.D.; Osgood, R.M. (October 1998). "A Progress Report on the Characterization and Modeling of a Very Flexible Turbine Design." Prepared for 1998 ASME Wind Energy Symposium, Reno, Nevada. January 11-14, 1999. NREL/CP-50025513. Golden, CO: National Renewable Energy Laboratory, 10 pp.

4. Kelley, N.D. (November 1992). "Full Vector (3-D) Inflow Simulation in Natural and Wind Farm Environments Using an Expanded Version of the SNLWIND (Veers) Turbulence Code." Prepared for $12^{\text {th }}$ ASME Wind Energy Symposium, January 31 February 4, 1993. NREL/TP-442-5225. Golden, CO: National Renewable Energy Laboratory, $8 \mathrm{pp}$.

5. Kelley, N.D.; Hand, M.; Larwood, S.; McKenna, E. (January 2002). "The NREL LargeScale Turbine Inflow and Response Experiment - Preliminary Results." Prepared for the $21^{\text {st }}$ ASME Wind Energy Symposium. Reno, Nevada. January 14-17, 2002. NREL/CP500-30917. Golden, CO: National Renewable Energy Laboratory, 15 pp.

6. Kelley, N.D.; Osgood, R.M.; Bialasiewicz; J.T.; Jakubowski, A (July-September 2000). "Using Wavelet Analysis to Assess Turbulence/Rotor Interactions." Wind Energy (3:3), pp. 121-134.

7. Wright, A.D.; Kelley, N.D. (1999). "Validation of a Model for a Two-Bladed Flexible Wind Turbine System." Proceedings of ASME/JSME Joint Fluids Engineering Conference. San Francisco, California. July 1999.

8. Kelley, N.D. (July 1999). "A Case for Including Atmospheric Thermodynamic Variables in Wind Turbine Fatigue Loading Parameter Identification." Prepared for the $2^{\text {nd }}$ Symposium on Wind Conditions for Wind Design - IEA Annex XI, April 12-13, 1999. NREL/CP-500-26829. Golden, CO: National Renewable Energy Laboratory, 16 pp.

9. Kelley, N.D.; Wright, A.D.; Buhl, M.L.; Tangler, J.L. (1996). "Long-Term Simulation of Turbulence-Induced Loads Using the SNLWIND-3D, FAST, YawDyn, and ADAMS Numerical Codes.” Prepared for 1997 ASME Wind Energy Symposium, Reno, Nevada. NREL/CP- 440-21673. Golden, CO: National Renewable Energy Laboratory, 14 pp.

10. Kelley, N.D.; Sutherland, H.J. (1996). "Damage Estimates from Long-Term Structural Analysis of a Wind Turbine in a U.S. Wind Farm Environment." Prepared for the 1997 ASME Wind Energy Symposium, Reno, Nevada. NREL/CP- 440-21672, Golden, CO: National Renewable Energy Laboratory. 12 pp. 
11. Smith, K.; Randall, G.; Malcolm, D.; Kelley, N.; Smith, B. "Evaluation of Wind Shear Patterns at Midwest Wind Energy Facilities." Proceedings American Wind Energy Association (AWEA) Windpower 2002 Conference. Portland, Oregon. June 2-5, 2002. $16 \mathrm{pp}$.

12. Eggers, A.J., Jr.; Digumarthi, R.; Cheney, K. (2003). "Wind Shear and Turbulence Effects on Rotor Fatigue and Loads Control.” Proceedings 2003 ASME Wind Energy Symposium. Reno, Nevada. January 6-9, 2003. 10 pp.

13. International Electrotechnical Commission. (1998). Safety of Wind Turbine Conversion Systems. 61400-1. Chicago, IL: International Electrotechnical Commission.

14. Bonner, W.D. (1968). "Climatology of the Low-Level Jet.” Monthly Weather Review (96), pp. 833-850.

15. Mitchell, M.K.; Arritt, R.W.; Labas, K. (September 1995). “An Hourly Climatology of the Summertime Great Plains Low-Level-Jet Using Wind Profiler Observations." Weather Forecasting (10), pp. 576-591.

16. Whiteman, C.D.; Bian, X; Zhong, S. (October 1997). "Low-Level Jet Climatology from Enhanced Rawinsonde Observations at a Site in the Southern Great Plains." J. Applied Meteorology (36), pp. 1363-1376.

17. Banta, R.M; Newsom, R.K.; Lundquist, J.K.; Pichugina, Y.L.; Coulter, R.L.; Mahrt, L.D. (2002). "Nocturnal Low-Level Jet Characteristics over Kansas during CASES-99." Boundary-Layer Meteorology (105), pp. 221-252.

18. Blackadar, A.K. (1957). "Boundary Layer Wind Maxima and Their Significance for the Growth of Nocturnal Inversions." Bulletin of the American Meteorological. Society (38), pp 283-290.

19. Lundquist, J.K. (2000). "The Evening Transition of the Atmospheric Boundary Layer: Inertial Oscillations and Boundary-Layer Dynamics.” Ph.D. Dissertation, University of Colorado. $180 \mathrm{pp}$.

20. Nappo, C.J. (2002). An Introduction to Atmospheric Gravity Waves. San Diego, CA: Academic Press.

21. Blumen, W.; Banta, R.M.; S.P. Burns, D. C.; Fritts, R. K.; Newsom, G.S.; Poulos, J. Sun. (2001). "Turbulence Statistics of a Kelvin-Helmholtz Billow Event Observed in the Nighttime Boundary Layer During the CASES-99 Field Program," Dynamics of Atmospheres and Oceans (34), pp. 189-204.

22. Bedard, A.J. Jr.; Canavero, F.; Einaudi, F. (December 1986). “Atmospheric Gravity Waves and Aircraft Turbulence Encounters." J. Atmospheric Sciences (43:23), pp. 28382844.

23. Einaudi, F.; Bedard, A.J. Jr.; Finnigan, J.J. (Feb 1989). "A Climatology of Gravity Waves and Other Coherent Disturbances at the Boulder Atmospheric Observatory during MarchApril 1984." J. Atmospheric Sciences (46:3), pp. 303-329.

24. A.J. Bedard, Jr. (1990). “A Review of Evidence for Strong, Small-Scale Vortical Flows During Downslope Windstorms." J. Wind Engineering and Industrial Aerodynamics (36), pp. 97-105. 
25. Cunningham, W.J. Jr.; Bedard, A.J. Jr. (Sept 1993). "Mountain Valley Evacuation by Upper Level Flows: A Scale Model Study.” AIAA Journal (31:9), pp. 1560-1573.

26. Sullivan, P.P.; McWilliams, Moeng, C.H. (1996). “A Grid Nesting Method for LargeEddy Simulation of Planetary Boundary Layer Flows.” Boundary-Layer Meteorology (80), pp. 167-202.

27. Werne, J.; Fritts, D.C. (February 15, 1999). "Stratified Shear Turbulence: Evolution and Statistics.” Geophysical Research Letters (26:4), pp. 439-442.

28. Lilly, D.K.; Zipser, E.J. (1972). "The Front Range Windstorm of 11 January 1972-A Meteorological Narrative." Weatherwise (25), pp. 56-63.

29. Lilly, D.K. (1978). “A Severe Downslope Windstorm and Aircraft Turbulence Event Induced by a Mountain Wave.” J. Atmospheric Sciences (35), pp. 59-77.

30. Banta, R.M.; Olivier, L.D.; Neff, W.D.; Levinson, D.H.; Ruffieux, D. (1995). "Influence of Canyon-Induced Flows on Flow and Dispersion over Adjacent Plains," Theoretical Applied Climatology (52), pp. 27-42.

31. Monti, P.; Fernando, H.J.S.; Princevac, M.; Chan, W.C.; Kowalewski, T.A.; Pardyjak, E.R. (2002). "Observations of Flow and Turbulence in the Nocturnal Boundary Layer over a Slope.” J. Atmospheric Sciences (59), pp. 2513-2534.

32. Stull, R.B. (1989). An Introduction to Boundary Layer Meteorology. Norwell, MA: Kluwer Academic Publishers.

33. Sutherland, H.J. (June 1999). On the Fatigue Analysis of Wind Turbines. SAND99-0089. Albuquerque, NM: Sandia National Laboratories.

34. Wilson, R.E.; Freeman, L.N.; Walker, S.N.; Harman, C.R. (September 1995). Users' Manual for the FAST_AD Advanced Dynamics Code. OSU/NREL Report 95-01.

35. Laino, D.J.; Hansen, A.C. (January 13, 2003). User's Guide to the Wind Turbine Dynamics Computer Program Version 12.14. Prepared for the National Renewable Energy Laboratory under Subcontract No. TCX-9-29209-01. Work performed by Windward Engineering, Salt Lake City, UT. (Available from the NWTC)

36. Malcolm, D.J.; Hansen, A.C. (August 2002). WindPACT Turbine Rotor Design Study. Worked performed by Global Energy Concepts, Kirkland, WA; Salt Lake City, UT: Windward Engineering, NREL/SR-500-32495. Golden, CO: National Renewable Energy Laboratory.

37. Jonkman, J. (2003). Personal communication. 


\section{REPORT DOCUMENTATION PAGE}

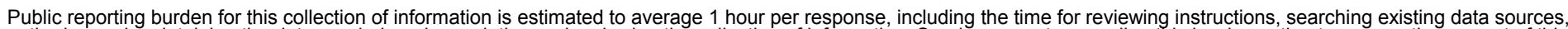

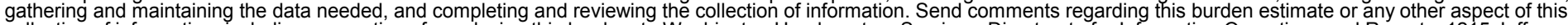

en to Washington Headquarters Services, Directorate

Davis Highway, Suite 1204, Arlington, VA 22202-4302, and to the Office of Management and Budget, Paperwork Reduction Project (0704-0188), Washington, DC 20503.
1. AGENCY USE ONLY (Leave blank)
2. REPORT DATE
January 2004
3. REPORT TYPE AND DATES COVERED
Technical Report

4. TITLE AND SUBTITLE

Lamar Low-Level Jet Project Interim Report

5. FUNDING NUMBERS

WER4.7001

6. $\mathrm{AUTHOR}(\mathrm{S})$

N. Kelley, M. Shirazi, D. Jager, S. Wilde, J. Adams, M. Buhl, P. Sullivan*, E. Patton*

7. PERFORMING ORGANIZATION NAME(S) AND ADDRESS(ES)

National Renewable Energy Laboratory

1617 Cole Blvd.

8. PERFORMING ORGANIZATION REPORT NUMBER

Golden, CO 80401-3393

NREL/TP-500-34593

*National Center for Atmospheric Research, Boulder, CO

9. SPONSORING/MONITORING AGENCY NAME(S) AND ADDRESS(ES)

10. SPONSORING/MONITORING AGENCY REPORT NUMBER

11. SUPPLEMENTARY NOTES

12a. DISTRIBUTION/AVAILABILITY STATEMENT

National Technical Information Service

U.S. Department of Commerce

5285 Port Royal Road

Springfield, VA 22161

13. ABSTRACT (Maximum 200 words)

This interim report presents the results to date from the Lamar Low-Level Jet Program (LLLJP) that has been established as joint effort among the U.S. Department of Energy (DOE), the National Wind Technology Center (NWTC) of the National Renewable Energy Laboratory (NREL), and General Electric Wind Energy (GE Wind). The purpose of this project is to develop an understanding of the influence of nocturnal low-level jet streams on the inflow turbulence environment and the documenting of any potential operating impacts on current large wind turbines and the Low Wind Speed Turbine (LWST) designs of the future. A year's record of detailed nocturnal turbulence measurements has been collected from NREL instrumentation installed on the GE Wind 120-m tower in southeastern Colorado and supplemented with mean wind profile data collected using an acoustic wind profiler or SODAR (Sound Detection and Ranging). The analyses of measurements taken as part of a previous program conducted at the NWTC have been used to aid in the interpretation of the results of representative case studies of data collected from the GE Wind tower.

14. SUBJECT TERMS

Lamar Low-Level Jet Program; LLLJP; U.S. Department of Energy; DOE; National

Wind Technology Center; NWTC; National Renewable Energy Laboratory; NREL;

General Electric Wind Energy; GE Wind; nocturnal low-level jet streams; inflow turbulence; Low Wind Speed Turbine; LWST

17. SECURITY CLASSIFICATION OF REPORT Unclassified
18. SECURITY CLASSIFICATION OF THIS PAGE Unclassified
19. SECURITY CLASSIFICATION OF ABSTRACT Unclassified
15. NUMBER OF PAGES

16. PRICE CODE

20. LIMITATION OF ABSTRACT

UL 